\title{
Step by step: \\ Engineering kinesins to study cargo trafficking in neurons
}




\section{Step by step:}

\section{Engineering kinesins to study \\ cargo trafficking in neurons}

\section{Stap voor stap:}

Het bouwen van kinesines voor het bestuderen van cargo transport in neuronen

(met een samenvatting in het Nederlands)

\section{Proefschrift}

ter verkrijging van de graad van doctor aan de Universiteit Utrecht op gezag van de rector magnificus, prof. dr. H. R. M. B. Kummeling, ingevolge het besluit van het college voor promoties in het openbaar te verdedigen op

woensdag 9 februari 2022 des ochtends om 10.15 uur

door

ISBN: 978-90-393-7438-2

The studies described in this thesis were performed at the division of Cell Biology at the Faculty of Science of Utrecht University in Utrecht, The Netherlands.

The printing of this thesis was financially supported by Alzheimer Nederland.

Cover design: Jessica Hummel

Layout: Jessica Hummel

Print: Ridderprint

Copyright $($ Jessica Janina Anna Hummel, 2022

All rights reserved

\section{Jessica Janina Anna Hummel}

geboren op 11 september 1992

te Zoetermeer 


\section{Promotor:}

Prof. dr. C. C. Hoogenraad

\section{Stap voor stap}

Wandel ik door het leven

Soms ga ik rechtdoor, dan weer langs kronkelende paden

Over hoge bergen en door diepe dalen

Zolang ik mijn eigen pad volg

Zal ik mijn bestemming vinden

\section{Stap voor stap}

Vind ik de weg naar mijn hart

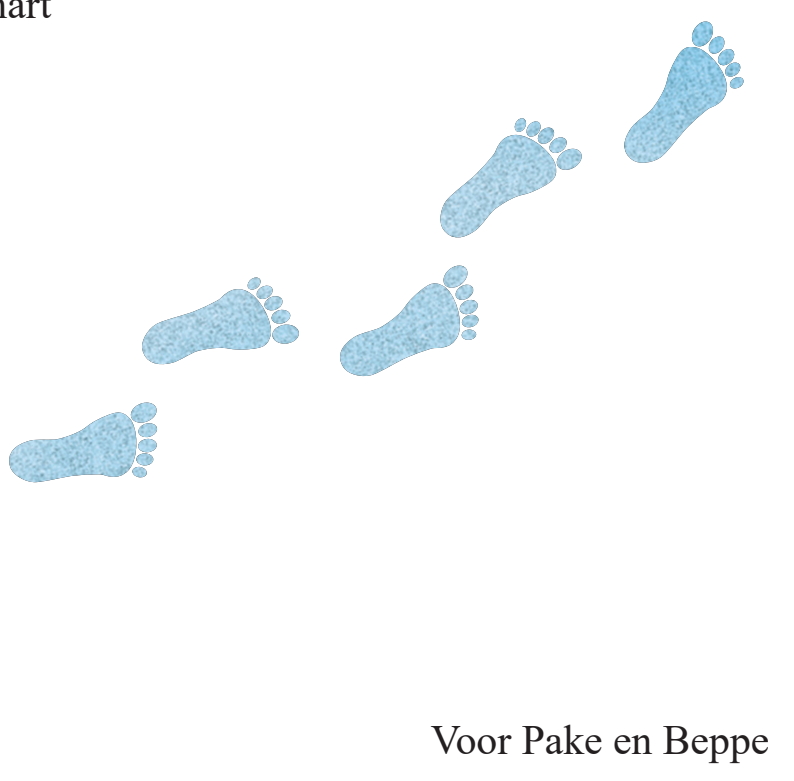




\section{Table of contents}

Chapter 1 General introduction

Chapter 2 The mechanism of kinesin inhibition by kinesin-binding protein

Chapter 3 Inducible manipulation of motor-cargo interaction using engineered kinesin motors

Chapter 4

Specific KIF1A-adaptor interactions control selective cargo recognition

Chapter 5

Combined kinesin-1 and kinesin-3 activity drives axonal trafficking of TrkB receptors in Rab6 carriers

Chapter 6

Addendum

Nederlandse samenvatting voor non-experts

Curriculum vitae

List of publications 


\section{General introduction}

Jessica J.A. Hummel ${ }^{1}$

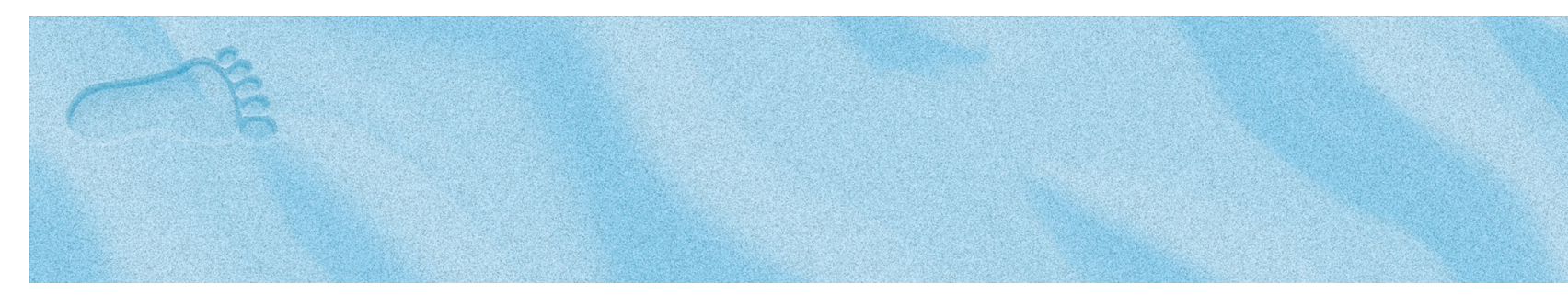

${ }^{1}$ Cell Biology, Neurobiology and Biophysics, Department of Biology, Faculty of Science, Utrecht University, 3584 CH Utrecht, the Netherlands. 


\section{Cell biology of the neuron}

The human brain is a complex organ consisting of a network of 100 billion neuronal cells (Azevedo et al., 2009). Individual neurons connect to form a neuronal circuit that fulfills a specific role within the brain network. Dysfunctional neurons result in disturbance of the circuitry, which disrupts the brain network and ultimately can lead to various neurological diseases (Stam, 2014). To better understand the complexity of our brain and to develop central nervous system (CNS) targeted medicines, we need to study the basic cell biology of a neuron.

\section{Neuronal polarity}

The most obvious feature of a neuronal cell is its polarized morphology. A neuron consist of a somatodendritic region with a cell body and multiple shorter processes, called dendrites, and one long, thin process, called the axon. This polarized morphology is required for signal transduction within the neuronal network. Dendritic spines, membranous protrusions found on dendrites, receive electrochemical signals from other cells, which are integrated in the somatodendritic region. The initial part of the axon consists of a specialized compartment, the axon initial segment (AIS), where an action potential is generated, propagated through the axon, and then transmitted to other cells at specialized contact points, ynapses, signal transduction between the pre-synaptic axonal site and post-synaptic dendritic spines is mediated by specific molecules called neurotransmitters. In response to the action potential, neurotransmitters are released from the presynaptic terminal and bind to their specific receptors on the membrane of the postsynaptic site. Neurons rely on their highly polarized morphology for many of their critical functions. As the axon and dendrites have a very distinct morphology, they need a specific set of building blocks, proteins, and organelles to function properly. To achieve a polarized distribution, neurons need a regulated delivery system with high spatiotemporal control. Active transport in axons and dendrites is mediated by molecular

\section{The neuronal cytoskeleton}

The neuronal cytoskeleton facilitates transport into specific cellular compartments and is important for the establishment of neuronal polarization and cell maintenance (Schelski Bradke, 2017; Hirokawa et al. 2010). The two man types of cytoskeleton are actin and Bradke, 2017; Hirokawa microtubules (MTs) (Kapitein and Hoogeraad, 2015, Luo, 2002). Actin filaments are pola polymers that are formed by two intertwined linear strands that fold into a right-handed helical twist with two dynamically different ends. Addition of new filamentous actin (F-actin) monomers occurs at the barbed (plus) end, whereas depolymerization takes place at the pointed (minus) end (Blanchoin et al., 2014; Pollard and Borisy, 2003). The actin cytoskeleton plays an important role in axon outgrowth, guidance, and branching (Luo, 2002). It is foun enriched in structures called filopodia and lamellipodia within growth cones of the axon (Dent et al, 2011). Furtermore, actin patches were found to be important for the architecture of the in dendritic pines (Hotulainen and Hoogenraad, 2010). Additionally, longitudinal actin fibers and periodic actin rings are found through both axons and dendrites and are thought to be important for ong-term axon and dendrite maintenance (Xu et al., 2013; Bär et al., 2016; Han et al., 2017). Microtubules are polar structures that are formed from polymerization of $\alpha$ - and $\beta$-tubulin. They form hollow cylinders with a highly dynamic nature, switching between phase of growth and disassembly (Nogales and Wang, 2006; Howard and Hyman, 2009). The slowe growing minus end exists of $\alpha$-tubulin subunits, whereas the fast growing, more dynamic plus ends are composed of $\beta$-tubulin (Akhmanova and Steinmetz, 2015). Additionally, the plus ends are decorated wit (Akhmova and Stein (Akhmanova and Steinmetz, 2008). MTs are crucial for intracellular transport and can induce morphological changes during neuronal development (Kapitein and Hoogenraad, 2015). In mammalian neurons, axonal MTs have a uniform plus end out orientation,
whereas MTs in dendrites have a mixed polarity (Baas et al., 1988; Yau et al., 2016).
The expression of different $\alpha$ - and $\beta$-tubulin isotypes and tubulin post-translational modifications (PTMs) generate a so called 'tubulin code', which provides a regulatory mechanism to control MT specialization (Janke, 2014; Park and Roll-Mecak, 2018) Additionally, MTs interact with microtubule-associated proteins (MAPs), which regulate MT function, dynamics, and cargo trafficking (Wieczorek et al., 2015; Chen et al., 1992).

\section{Motor proteins}

Motor proteins regulate active transport of various proteins, organelles, and other cellular components with high spatiotemporal control. There are three types of motor proteins: myosin, dynein, and kinesin. These motors typically contain a motor domain that uses the energy from ATP hydrolysis to walk along the cellular cytoskeleton, and a tail domain that whereas dynein and kinesins move along MTs (Hirokawa et al., 2010). The precise interplay between the various motors is crucial for neuronal functioning.

\section{Myosin motors}

The actin-based myosin motor family contains 40 human myosin genes that have been classified into 12 families (Berg et al., 2001). In principle myosin motors move toward the plus ends of actin filaments, except for class VI myosins, which are minus end directed (Robert-Paganin et al., 2020; Wells et al., 1999). The motor domain of myosin is responsible for actin binding and generation of movement, whereas the cargo-binding tail domain determines its functional specificity (Lu et al., 2014; Robert-Paganin et al., 2020). The motor and cargo-binding domain are linked by a neck linker that varies in length within different myosins and functions as a lever arm (Fig 1A). Its length together with the degree of rotation determines the myosin stepping size (Köhler et al., 2003; Purcell et al , 2002). Myosin-based Kneussel and Wagne, 2013, Bridgman, 2004). Furthermore, contractile myosin II interacts with actin to form actomysosin rings, which are required for AIS assembly and regulate the distribution of AIS components along the axon (Berger et al., 2018).

\section{Kinesin motors}

Within the kinesin superfamily 45 different mammalian genes have been identified, which are classified into 14 families (Miki et al., 2001; Lawrence et al., 2004). The general structure of kinesin motors consists of a motor and cargo-binding domain, which are linked by a shor neck linker and a stalk domain (Hirokawa et al., 2009; Kato et al., 2018). The stalk region contains several coiled-coil domains that are involved in motor regulation, for example by autoinhibition or dimerization mechanisms. There are three distinct kinesin types: $\mathrm{N}-, \mathrm{M}-$, and C-kinesins (Hirokawa et al., 2009). Kinesin family 1-12 belong to the N-kinesins. They have their motor domain at the N-terminus and walk towards MT plus ends (Fig 1B) (Wozniak et al., 2004). In contrast, C-kinesins in the kinesin-14 family, have their motor domain at the C-terminus and move towards MT minus ends (Endow, 1999). Finally, M-kinesins from the kinesin-13 family have an internal motor domain. These unconventional kinesins do not move kinesin-13 family have an internal motor domain. These unconventional kinesins do not move
along MTs but are involved in MT depolymerization (Wordeman, 2005). Kinesin motors are along MTs but are involved in MT depolymerization (Wordeman, 2005). Kinesin motors are crucial for neuronal cargo trafficking, but also play an important role in the establishment of neuronal polarity (Lu and Gelfand, 2017; Hirokawa et al., 2010). N-kinesins walk along neuronal MTs in a hand-over-hand manner with a low side-stepping frequency (Yildiz et al., kinesins of the same or different families, which increases the run length of cargo and is thought to enable cargo to overcome macromolecular crowding (Furuta et al, 2017; Ando (2014; et al., 2014, Beeg et al., 2008, Gross et al., 2007). For example, combined activity of the kinesin-1 motor KIF5 and the kinesin-3 motor KIF1A was found to regulate trafficking of TrkB vesicles, which will be further discussed in Chapter 5. 


\section{Dynein motor}

Cytoplasmic dynein regulates intracellular cargo trafficking as this motor walks along MTs towards their minus ends. Dynein consists of a large protein complex that contains two heavy chains, which are involved in generation of movement, two intermediate chains, four light intermediate chains and several light chains (Fig 1B) (Kevin Pfister et al., 2005; Roberts et al 2013). Dynein activity and binding capacity for cargo is regulated by an associated protein complex, dynactin (Schroer, 2004). In addition, other adaptor proteins, such as Bicauda D, regulate dynein motility and processivity (Hoogenraad et al., 2003; McKenney et al., 2014). Cytoplasmic dynein is the main driver of retrograde transport in the axon (Hirokawa and Takemura, 2005). Furthermore, it was found to be important for axon elongation and outgrowth, and for dendritic branching (Grabham et al., 2007; Roossien et al., 2014; Tempes et al., 2020). As for kinesins, co-operation between dynein motors facilitates robust retrograde axonal transport and it was found that endosomal vesicles can recruit up to seven dynein

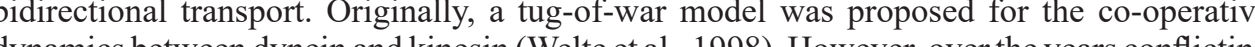
dynamics between dynein and kinesin (Welte et al., 1998). However, over the years conflicting results have been found and new models for bidirectional transport have been proposed, including microtubule tethering, mechanical activation, and steric disinhibition (Hancock, 2014). Future biochemical experiments and mathematical modelling should further elucidate the exact mechanism of how kinesin and dynein mediate bidirectional cargo transport.

A

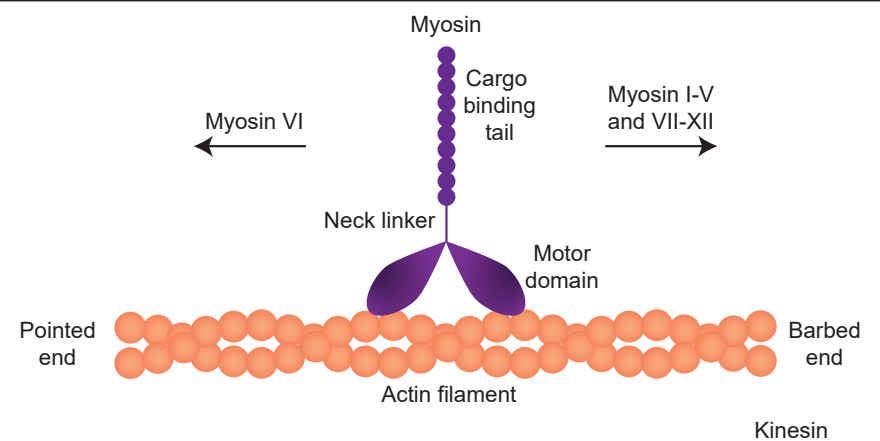

B

Dynein

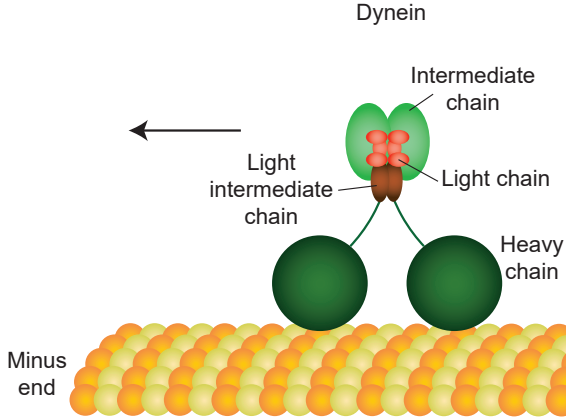

1. Structure of myosin, dynein, and kinesin

Figure 1. Structure of myosin, dynein, and kinesin

(A) Schematic representation of the structure of myosin motors. The cargo binding tail is linked to the motor domain via a neck linker that varies between different myosins. The motor domain moves along actin filaments towards the barbed (plus) end, except for myosin VI that moves towards the pointed (minus) end. (B) Schematic representation of the structure of dynein and kinesin. Dynein consists of two heavy chains, two intermediate chains, four light intermediate chains, and several light chains and moves along the MT towards its minus end. Kinesin consists of cargo-binding domain that is linked via a stalk domain to the motor domain, which walks towards the MT plus end.

\section{Regulation of transport by kinesins}

Regulation of motor-based cargo trafficking with high spatial and temporal control is essential to ensure precise cargo delivery in neurons. The large number of different kinesin genes allows different kinesins to confer specific transport parameters. To achieve a controlled transport network, individual motor proteins need to be tightly regulated. Different motors have specific mechanisms that regulate their activity and cargo-binding properties and thereby create unique transport properties. Here, we will highlight several regulation mechanism of the kinesin- 1 to kinesin- 4 family, as these are the main transporting kinesins in cells.

\section{Regulation by kinesin's dynamic structure}

Kinesin motors contain a motor domain that is linked to a cargo-binding domain by a short neck linker (Wang et al., 2015). They can form monomers and dimers (Marx et al., 2009) and interact with other co-factors to form multimeric complexes. Most kinesins contain a dimeric motor head and when active these processive dimers move along the MT lattice in a hand-over-hand manner (Yildiz et al., 2004). The kinesin-1 family motors have well been studied and were found to exist as a heterotetramer consisting of a homodimer of kinesin ight chain (KLC), which forms the cargo-binding tail (DeBoer et al., 2008). The kinesin-2 family members also exist as homodimers, but KIF3 can also form a heterotrimeric complex containing a heterodimeric kinesin head with one KIF3A and one KIF3B subunit together with the KAP adaptor (Scholey, 2013). In the kinesin-3 family both monomeric and dimeric motors were found. For monomeric kinesins contrasting results were obtained regarding their processive motility (Xie, 2010). Initially, studies have suggested non-processive motility for single-headed kinesins (Young et al., 1998; Kamei et al., 2005), but processive movement of monomeric KIF1A was observed as well (Okada et al., 2003). Later it was found that on the motor-cargo distance and the cargo load, and monomers become less efficient as the length of the stalk increases, requiring dimerization to pull against load (Schimert et al., 2019). It was also shown that dimerization of kinesin-3 motors results in superprocessive motility (Soppina et al., 2014), thereby suggesting that motor dimerization provides a mechanism to regulate motor activity. This might be a unique feature for this kinesin family, as other kinesin complexes already contain a homodimeric motor head and therefore lack such additional layer of regulation. Thus, kinesin dimerization and the formation of kinesin transport complexes can regulate motor activity (Fig 2A)

For members of the kinesin-1, kinesin-2, and kinesin-3 families autoinhibition of the motor head by the cargo-binding tail domain is a common regulating mechanism to tightly control motor activity in the absence of cargo (Fig 2A) (Verhey and Hammond, 2009). The molecular mechanism of kinesin- 1 autoinhibition has been studied extensively and it was found that in the inactive state the tail region folds back on the motor domain, thereby preventing processive motor movement (Dietrich et al., 2008; Kaan et al., 2011; Coy et al., 1999). Removal of the cargo binding tail domain, results in a constitutively active motor, thereby supporting a regulatory autoinhibition mechanism (Friedman and Vale, 1999 Jacobson et al., 2006). Interaction with the KLC domains provides a second inhibition site and it was found that in its inactive form the $\mathrm{KHC}$ motor domains are pushed apart by the KLC domains. In the active form, when the interaction with the KLC domains is disrupted, the KHC domains are closer together. Thus kinesin-1 activation requires two conformational changes, one that separates the KHC motor and tail domains and one that moves the $\mathrm{KHC}$ motor domains closer together (Cai et al., 2007a). For the kinesin-3 family members an importan role for the CC1 domain in autoinhibition has been suggested. In both KIF1A and KIF13B removal of this domain results in a constitutively active motor, thereby suggesting a role of this domain in autoinhibition of the motor domain (Ren et al., 2018; Hammond et al., 2009). 
To date, less is known about autoinhibition mechanisms in the kinesin- 2 and kinesin- 4 families. For kinesin-2 family member KIF17 a similar autoinhibition mechanism has been suggested, where a non-motor region folds back on the motor and autoinhibition is relieved by cargo binding (Hammond et al., 2010) and KIF3A was found to exist in an autoinhibited state (Chen et al., 2018). An autoinhibition mechanism has also been described for kinesin-4 family member KIF21A (van der Vaart et al., 2013). Furthermore, MT binding by the unconventional KIF7 motor was found to be regulated by autoinhibition (Blasius et al., 2019). To summarize, the specific features of kinesins structure and conformational changes within are important regulatory mechanisms mediating kinesin motor activity (Fig 2A).

\section{Regulation by cargo binding and co-factors}

Relieve of kinesin autoinhibition and subsequent motor activation is generally achieved by competitive binding of a cargo vesicle to the cargo-binding tail domain (Verhey and Hammond, 2009). The kinesin-cargo interaction can be facilitated by additional co-factors, such as scaffolding or adaptor proteins, that bind both the kinesin cargo-binding tail domain and proteins on the cargo membrane and thereby mediate the relieve of autoinhibition (Fig 2B). For kinesin-1 family members it was found that two binding partners, c-Jun N-termina kinase-interacting protein 1 (JIP1) and fasciculation and elongation protein zetal (FEZ1), are required to relieve the double autoinhibited state and activate MT binding and motility (Blasius et al, 2007). In line with this, it was found that cacto binding to KLC mediated by ( JP1 and JiP3 Relieve of autoinhibition by binding to cargo or co-factors was also observed in the kinesin-3 family. Cargo binding to monomeric KIF1A and subsequent relieve of autoinhibition, enables motor dimerization and results in an active motor (Soppina et al., 2014). Later, it has been suggested that binding of calmodulin $(\mathrm{CaM})$ to the KIF1A stalk domain results in a conformational change that is required to enable cargo binding and further motor activation (Stucchi et al., 2018). For dimeric KIF1C it was shown that binding of cargo via the adapto Hook3 as well as binding to protein tyrosine phosphatase N21 (PTPN21), a regulatory protein,

Scaffolding and adaptor proteins bind kinesins and act as binding platforms to mediate protein-protein interactions and facilitate cargo trafficking by regulating motor activity ( $\mathrm{Fu}$ and Holzbaur, 2014; Hirokawa et al., 2009). Next to their role in mediating the kinesincargo interaction, they interact with other adaptor or signaling proteins, such as kinases or GTPases, which allows them to integrate signaling proteins and regulate cargo transport. A coordination model to describe the co-operative dynamics between kinesin and dynein hypothesizes that kinesin and dynein are both bound to the cargo vesicle, and that activity of a specific motor is regulated by scaffolding proteins (Fu and Holzbaur, 2014). For example, this is observed for autophagosomes, which bind to both dynein and kinesin, but in neuron axons only show fast dynein-mediated retrograde thans axom caffolding proten $J$, which was found mechanisms and by binding to the autophagosome adaptor LC3 (Fu et al., 2014; Maday et al., 2012). Adoptor proteins may also provide a way to achieve specificity of certain kinesin for a cargo. For example, it was found that mitochondria are transported by both kinesinfamily KIF5 motors and kinesin-3 family KIF1B $\alpha$ motors. This interaction is regulated by different adaptor proteins, namely Milton and Miro for KIF5 and KBP for KIFIB $\alpha$ (Wang an Schwarz, 2009; Wozniak et al., 2005), thereby suggesting that the specific adaptor determines which kinesin motor is recruited. Interestingly, KBP inhibits other kinesins, including kinesin-3 family member KIF1A, showing that co-factors have different functions in the inhibitory KBP-kinesin interaction will be discussed in Chapter 2. Different cargos have been described for KIF1A, including dense core vesicles (DCVs), lysosomes and synaptic vesicles (SVs) (Okada et al., 1995; Guardia et al., 2016; Lo et al., 2011), as well as various adaptors, such as liprin- $\alpha$, Arl8, and DENN/MADD (Shin et al., 2003; Niwa et al., 2008, 2016).

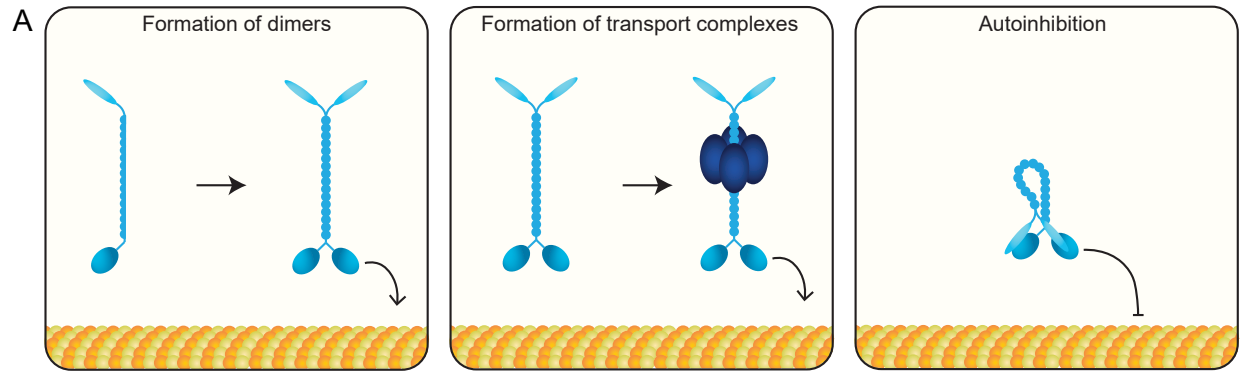

$\mathrm{B}$
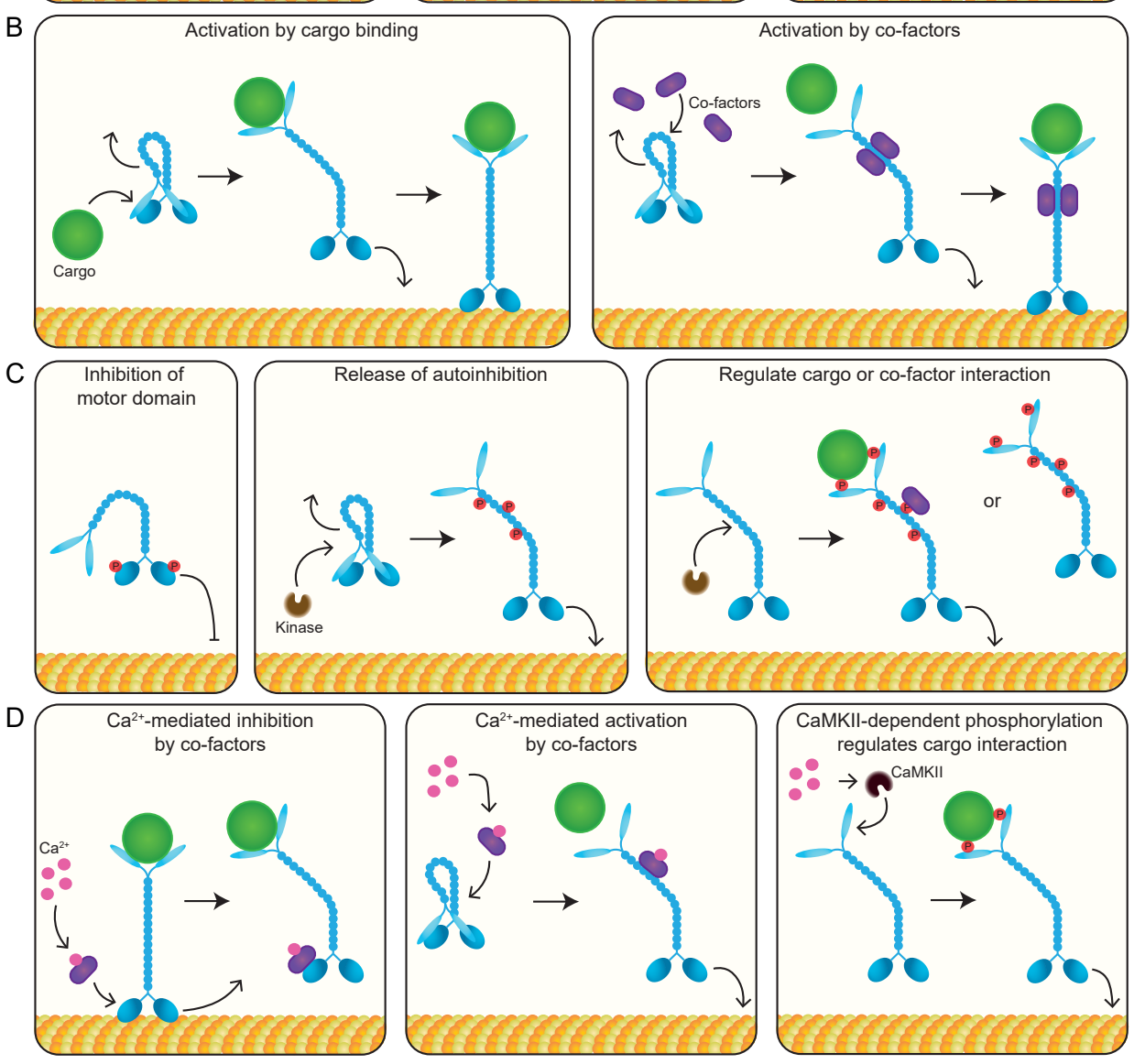

Figure 2. Kinesin regulatory mechanism

(A) Regulation of kinesin motor activity by kinesins dynamic structure. Kinesin dimerization (left) or formation of kinesin transport complexes (middle) can be required for kinesin activity. Kinesins can also exist in an autoinhibited state in which the tail domain is folded back on the motor domain, thereby preventing association with MTs and be relieved by cargo binding to the kinesin cargo-binding domain, thereby allowing MT-based motor movement (left). Binding of co-factors to kinesins stalk domain also releases autoinhibition, thereby enabling the cargo-binding domain to interact with cargo and the motor domain to facilitate MT-based cargo transport. (C) Regulation of kinesin motor activity by phosphorylation. Phosphorylation can directly regulate the kinesin motor domain (left) or regulate the release of autoinhibition (middle). Phosphorylation of the stalk or cargo-binding domain can mediate the interaction with cargo or co-factors (right). (D) Regulation of kinesin motor activity by neuronal activity. Increased calcium levels can regulate co-factors to bind the kinesin motor domain, thereby inhibiting transport (left) Increased calcium levels also activate CaMKII to phosphorylate kinesins and enable cargo transport (right). 
Chapter 4 describes how different adaptors mediate the specificity of KIF1A for a cargo. Adaptor proteins can also integrate cellular signals, thereby regulating the cargo trafficking machinery and influencing neuronal development and function. For example, TRIM46 transport into the future axon has been suggested to be driven by the KIF3A/B/KAP complex. MARK2-dependent phosphorylation of KAP disiopts the interaction with TRIM46, the preventing TRIM46 to enter the somatodenritic area which is under high MARK2 activity and mediates its localization into the future AIS (Ichinose et al., 2019). These examples show that the interaction between kinesin and cargo or other co-factors provides importan regulatory mechanisms for kinesin activity.

\section{Regulation by signaling mechanisms}

Kinesin motor activity is also regulated by protein modifications, including phosphorylation and responses to neuronal activity which is sensed by increased calcium levels. Phosphorylation mechanisms are found at different levels and can directly regulate motor activity or can be involved in the cargo interaction, thereby indirectly regulating motor processivity. Various phospho-regulatory mechanisms have been identified for kinesin-1. For example, it was found that phosphorylation of the motor domain by JNK3 directly affects kinesin motility parameters (DeBerg et al.,2013). Additionally, phosphorylation of KLC mediates inesins cargo-binding prop (h) phosphorylation of KLC by glycogen synthase kinase 3 (GSK3) negatively affects cargo binding and transport, without changing kinesin MT binding and ATPase activities. These observations suggest that phosphorylation provides a mechanism for cargo release in specific subcellular domains with high GSK3 activity (Morfini, 2002). Furthermore, phosphorylatio of a threonine residue, Thr466, in KLC1 disrupts the interaction with JIP1 and was found to impair transport of its cargo, amyloid $\beta$-protein precursor (APP) vesicles (Chiba et al., 2017). Interestingly, phosphorylation of a serine residue, Ser460, in KLC1 is involved in calsyntenin-1 transport (Vagnoni et al., 2011), suggesting that phosphorylation at specific ites might provide a mechanism for cargo selectivity. Additionally, phospho-regulation of chesulation of adaptor proteins the kinesin-1 adaptor FEZ1 is required for its interaction with kinesin and thereby regulate trafficking of syntaxin la (Chua et al., 2012). Phospho-regulatory mechanisms have also been described for cargo trafficking by the kinesin-2 family members. For KIF3A it was shown that phosphorylation of Ser689 induces conformational changes that relieve autoinhibition, thereby directly regulating motor activity (Chen et al., 2018). It was also found that phosphorylation of KIF17 by CaMKII disrupts its interaction with adaptor Mint1 and results in cargo release (Guillaud et al. 2008). Within the kinesin-3 family, little is known about Ser1092 is required for its interaction with the adaptor protein 14-3-3, thereby regulating cargo interactions (Dorner et al., 1999). Furthermore, GSK3 $\beta$ has been implicated in cargo transport by KIF1A. However, this does not involve phosphorylation of the kinesin moto domain and the exact mechanism of regulation needs to be investigated (Gan et al., 2020). In Chapter 4 we describe that phosphorylation of S1665 in the PH domain of KIF1A is critical for the cargo interaction. Thus, phosphorylation can regulate motor activity by directly inhibiting the motor domain, by regulating autoinhibition or by regulating the interaction with cargo and co-factors (Fig 2C).

Neuronal activity is another important signal that regulates cargo trafficking, as this requires specific cargo to be at the right subcellular compartment for proper neuronal signaling, and several calcium-dependent mechanisms for motor activity have been described. For example, mitochondrial transport by kinesin-1 is halted upon increased $\mathrm{Ca}^{2+}$ levels. It was shown that calcium binding to the mitochondrial adaptor Miro permits Miro to interact with the kinesin motor domain, thereby preventing the interaction with MTs and arresting mitochondrial motility (Wang and Schwarz, 2009).
For transport of AMPA receptors by kinesin-1 a different mechanism was found, in which neuronal activity leads to activation of CaMKII, and subsequent phosphorylation of the kinesin motor domain enables cargo trafficking (Hoerndli et al., 2015). A similar mechanism of activity-dependent cargo loading via kinesin motor phosphorylation by CaMKII has been 15). For kinesin-3 family member KIF1A it was fou hat increased calcium levels enable binding of $\mathrm{CaM}$ to the KIF1A stalk domain, thereby relieving autoinhibition and activating cargo transport (Stucchi et al., 2018). In Chapter 4 we propose a mechanism for KIF1A motor activation, where CaM activates CaMKII to phosphorylate the KIF1A PH domain, which enables interaction with a cargo vesicle. KIF21B, a kinesin- 4 family member, was found to execute two distinct functions that are regulated by neuronal activity. First, it is involved in retrograde brain-derived neurotrophic factor (BDNF)TrkB complexes. Second, it regulates MT dynamics via a non-motor MT-binding domain It was shown that increased neuronal activity enhances KIF21B motility at the expense of (t) Thus, responses of kinesin to neuronal activity and related signaling cascades are important for proper regulation of the trafficking machinery (Fig 2D).

\section{Methods to study cargo trafficking}

Cargo trafficking in neurons requires tight regulation of motor proteins with high spatiotemporal control. Structural studies have provided much knowledge into the kinesin structure and into the mechanism of ATP hydrolysis and processive movement (Wang et al. 2015). However, studying the specific regulation mechanisms of each kinesin in the neuronal context has proved to be a challenging task. Over the years, several tools have been developed to investigate the regulation mechanisms of kinesin motor proteins and to gain more insights into the complex neuronal transport machinery.

\section{Studying kinesin motor function}

Single-molecule imaging techniques have been used to study kinesin motor behavior and these in vitro experiments provided detailed information about the kinetics of kinesi translocation (Belyy and Yildiz, 2014; Yildiz et al., 2004; Vale et al., 1996). However, the information gathered from such assays is difficult to translate to kinesin movement in living cells, as kinesins in a cellular environment are facing many additional layers of regulation. Despite recent optimizations of these assays, such as the inclusion of different tubulin PTMs and isoforms (Sirajuddin et al., 2014; Kaul et al., 2014) and analysis in mammalian cells (Cai et al., 2007b, 2009), further research into kinesin motor activity in a neuronal context is required. An efficient method to study kinesin motor behavior in hippocampal neurons involves the generation of constitutively active motors by removal of the autoinhibiting tail involves the generation of constitutively active motors by removal of the autoinhibiting tail
domain. Such assays can provide information about kinesin distribution in specific cellular compartments and the influence of tubulin modifications on motor translocation. In addition, the efficiency of kinesin movement can be assessed, although with less precision then from in vitro assays (Yang et al., 2016). However, as these truncated motors lack the tail domain and are incapable of cargo binding, no further conclusions about cargo distribution should be made. This is well illustrated by the discrepancy in motor translocation found for KIF13 KIF13B motor domain translocated into both axons and dendrites. Expression of full-length motors showed that KIF13Alabeled vesicles are transported into both axons and dendrites, whereas KIF13B labeled vesicles are polarized to dendrites, suggesting additional layers of regulation by the cargo-binding tail domains (Yang et al., 2016; Jenkins et al., 2012). Inducible assays in which a kinesin motor is artificially linked to a cargo, provide another method to study kinesin motor behavior. For example, a rapalog-inducible heterodimerization system was developed to identify kinesin motors that can drive cargo transport in cells and to investigate the polarized trafficking pattern of each transporting kinesin (Lipka et al., 2016) 
A similar system was used to study the inhibitory effect of kinesin-binding protein (KBP) on the motor activity of different kinesins (Kevenaar et al., 2016), showing the potentia of such assays to elucidate kinesin regulatory mechanisms. Further application of this assay is described in Chapter 2, where it is combined with biochemical assays and cryo-

electron microscopy to unravel the interaction modes between KBP and KIF15 or KIF1A.

\section{Investigating kinesin-cargo interactions}

Next to the regulation of motor activity, proper cargo transport depends on the kinesincargo interaction mode. The development of yeast two-hybrid systems has provided a robust method to identify protein-protein interactions (Fields and Song, 1989). Using this technique, several kinesin-cargo interactions have been identified, including the interaction between KIF5 and JIPs, and KIF1B $\alpha$ and KBP (Verhey et al., 2001; Wozniak et al., 2005). Despite optimalization of the yeast two-hybrid system over the years (Brückner et al., 2009), the assay is limited by the absence of a native cellular environment and therefore misses the kinetics, thermodynamics, stoichiometry, and cofactors that might influence the kinesin-cargo interaction. For example, it was found that the interaction between kinesin-1 and JIP1 depends on which protein binds to JIP1 (Satake et al., 2013), and additional techniques are required to further unravel the interactions picked up in yeast two-hybrid screens. Another approach is to disrupt kinesin motor protein function by introducing mutations or RNA interference and examine the changes in cargo proteins. For example, mutation studies were used to identify DCVs and SVs as cargos for KIF1A in flies (Barkus et al., 2008). In addition, RNA interference experiments in hippocampal neurons showed defects in DCV and SV transport upon KIF1A knock down (Stucchi et al., 2018; Kevenaar et al., 2016), confirming the role of KIF1A in transport of these cargos. However, as cargos are often transported by multiple motors, cells might have developed compensatory mechanisms to drive transport and false negatives can easily arise. Recently, a split kinesin method based on the chemically inducible linkage of a kinesin tail domain to the constitutively active, axonal KIF5C motor domain (Jenkins ., 2012). This provides an elegant method to study kinsin-cargo interactions but is limited by its dependence on single cell imaging, making the experiments technically challenging and time consuming. In Chapter 3, we describe the development and optimalization of an engineered motor platform, which was based on the split kinesin approach, to study kinesincargo interactions and to manipulate polarized cargo trafficking (Fig 3). This platform provides a robust tool to study kinesin motor function and has a wide range of future applications.

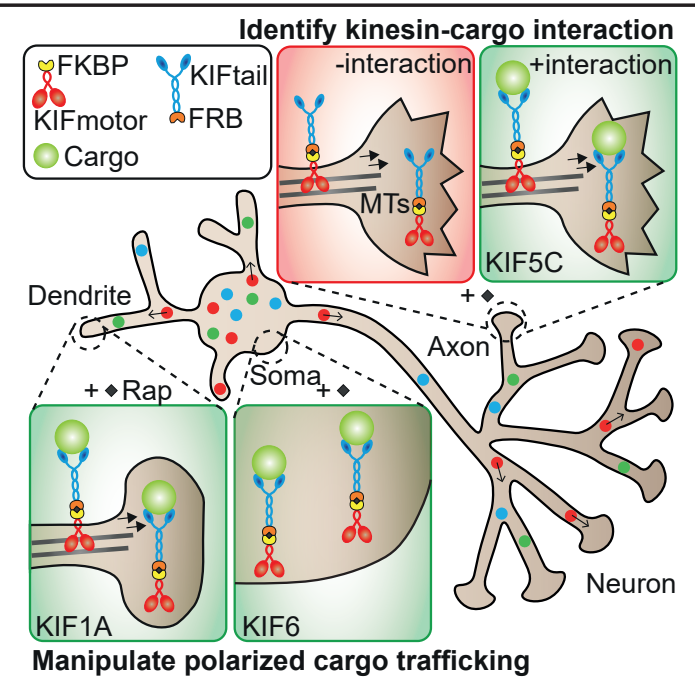

Figure 3. The engineered motor toolbox Schematic representation of the engineered motor toolbox. A kinesin motor domain is fused to a FKBP domain, and a kinesin tal frapalog results in the formation of a fill kinesin. When using the constitutively active $\mathrm{KIF} 5 \mathrm{C}$ motor that translocates into the axon, the toolbox enables investigation of the interaction between a specific kinesin tail domain and a cargo, as a clear re-localization of cargo into the axon will be observed in case of an interaction. The toolbox also allows manipulation of polarized cargo trafficking, by using the motor dinain or KIFlA to transpot caf KIF6 to capture cargo in the cell soin.

Manipulate polarized cargo trafficking

\section{Scope of this thesis}

Kinesin motor proteins facilitate polarized cargo trafficking in neurons, which is require for proper neuronal development and functioning. In this thesis, we developed and used engineered kinesin motors to study and elucidate the molecular mechanisms of cargo trafficking in neurons.

In Chapter 2, we investigated the KBP-KIF15 interaction interface using a combination of crystal structure analysis and cellular assays. Cryo-electron microscopy was used to determine the structure of KBP alone and when complexed with the KIF15 moto domain. Structure analysis revealed several interaction domains in KBP involved in motor binding. The importance of these domains for interaction with KIF15 and KIF1A was further validated using cellular translocation assays and biochemical binding assays.

Chapter 3 describes the development of an engineered motor platform to study kinesin motor proteins. This platform provides a highly adaptable and robust tool to study kinesin-cargo binding as well as to manipulate cargo trafficking. We use our tool to identify all kinesins that interact with DCVs and transferrin receptor (TfR) containing endosomes, to identify cargo for the KIF1A motor, and to determine the minimum binding domain of KIF16B required for cargo binding. Furthermore, we show that the platform can be used to steer cargo specifically into dendrites or axons, thereby manipulating the polarized trafficking machinery.

The subsequent chapters describe how we used our engineered motor platform in combination with other techniques to further study molecular mechanisms of cargo trafficking. In Chapter $\mathbf{4}$ we describe the regulation of cargo transport by different KIF1A domains. We show that the $\mathrm{CC} 1$ and $\mathrm{CC} 2$ domains are involved in motor activation by autoinhibition and dimerization mechanisms. The $\mathrm{CC} 3$ and $\mathrm{PH}$ domains are involved in cargo binding via interactions with specific adaptors and a phosphorylation mechanism. Chapter 5 focuses on TrkB signaling and with the use of engineered motors we show that KIF5C and KIF1A cooperate to regulate axonal transport of TrkB carriers.

In Chapter 6, we summarize the results described in chapters 2 to 5 and discuss them in a broader perspective to highlight remaining questions and future research directions. 
REFERENCES

Akhmanova, A., and Steinmetz, M.O. (2008). Tracking the ends: A dynamic protein network controls the fate of microtubule tips. Nat. Rev. Mol. Cell Biol. 9, 309-322.

Akhmanova, A., and Steinmetz, M.O. (2015). Control of microtubule organization and dynamics: Two ends in the

Ando, D., Mattson, M.K., Xu, J., and Gopinathan, A. (2014). Cooperative protofilament switching emerges from Azevedo, F.A.C., Carvalho, L.R.B., Grinberg, L.T., Farfel, J.M., Ferretti, R.E.L., Leite, R.E.P., Filho, W.J., Lent, R., and Herculano-Houzel, S. (2009). Equal numbers of neuronal and nonneuronal cells make the human brain an isometrically scaled-up primate brain. J. Comp. Neurol. 513, 532-541. Baas, P.W., Deitch, J.S., Black, M.M., and Banker, G.A. (1988). Polarity orientation of microtubules in hippocampal
neurons: Uniformity in the axon and nonuniformity in the dendrite. PNAS 85, 8335-8339. Bär, J., Kobler, O., Van Bommel, B., and Mikhaylova, M. (2016). Periodic F-actin structures shape the neck of dendritic spines. Sci. Rep. 6, 1-9.

Barkus, R. V, Klyachko, O., Horiuchi, D., Dickson, B.J., and Saxton, W.M. (2008). Identification of an axonal kinesin-3 motor for fast anterograde vesicle transport that facilitates retrograde transport of neuropeptides. Mol.

Beeg, J., Klumpp, S., Dimova, R., Gracià, R.S., Unger, E., and Lipowsky, R. (2008). Transport of beads by several

Belyy, V., and Yildiz, A. (2014). Processive cytoskeletal motors studied with single-molecule fluorescence Berg, J.S., Powell, B.C., and Cheney, R.E. (2001). A millennial myosin census. Mol. Biol. Cell 12, 780-794. Berger, S.L., Leo-Macias, A., Yuen, S., Khatri, L., Pfennig, S., Zhang, Y., Agullo-Pascual, E., Caillol, G., Zhu,
M.S., Rothenberg, E., et al. (2018). Localized myosin II activity regulates assembly and plasticity of the axon initial egment. Neuron $97,555-570$

Blanchoin, L., Boujemaa-Paterski, R., Sykes, C., and Plastino, J. (2014). Actin dynamics, architecture, and

Blasius, T.L., Cai, D., Jih, G.T., Toret, C.P., and Verhey, K.J. (2007). Two binding partners cooperate to activate the
molecular motor Kinesin-1. J. Cell Biol. 176, 11-17. Blasius, T.L., Yue, Y., and Verhey, K. (2019). Microtubule binding of the kinesin-4 KIF7 and its regulation by
autoinhibition. BioRxiv.

Bridgman, P.C. (2004). Myosin-dependent transport in neurons. J. Neurobiol. 58, 164-174

Brückner, A., Polge, C., Lentze, N., Auerbach, D., and Schlattner, U. (2009). Yeast two-hybrid, a powerful tool for systems biology. Int. J. Mol. Sci. 10, 2763-2788.

Cai, D., Hoppe, A.D., Swanson, J.A., and Verhey, K.J. (2007a). Kinesin-1 structural organization and conformational
changes revealed by FRET stoichiometry in live cells. J. Cell Biol. 176, 51-63. changes revealed by FRET stoichiometry in live cells. J. Cell Biol. 176, 51-63.

Cai, D., Verhey, K.J., and Meyhöfer, E. (2007b). Tracking single kinesin molecules in the cytoplasm of mammalian

Cai, D., McEwen, D.P., Martens, J.R., Meyhofer, E., and Verhey, K.J. (2009). Single molecule imaging reveals

Chen, J., Kanai, Y., Cowan, N.J., and Hirokawa, N. (1992). Projection domains of MAP2 and tau determine spacings
between microtubules in dendrites and axans. Nature 360, 674-677. Chen, K., Saw, W., Lama, D., Verma, C., Grüber, G., and Koh, C. (2018). Phosphorylation regulates auto-inhibition Chiba, K., Chien, K.Y., Sobu, Y., Hata, S., Kato, S., Nakaya, T., Okada, Y., Nairn, A.C., Kinjo, M., Taru, H., et a (2017). Phosphorylation of KLC1 modifies interaction with JIP1 and abolishes the enhanced fast velocity of APP

transport by kinesin-1. Mol. Biol. Cell 28, 3857-3869.
Chowdary, P.D., Che, D.L., Kaplan, L., Chen, O., Pu, K., Bawendi, M., and Cui, B. (2015). Nanoparticle-assisted optical
tethering of endosomes reveals the cooperative function of dyneins in retrograde axonal transport Sci. Rep. 5, 1-11.

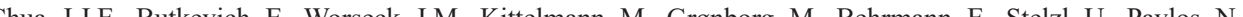
Lalowski, M.M. Eimer, S., et al. (2012). Phosphorylation-regulated axonal dependent transport of syntaxin 1 is mediated by a Kinesin-1 adapter. PNAS 109, 5862-5867. Cockburn, J.J.B., Hesketh, S.J., Mulhair, P., Thomsen, M., O'Connell, M.J., and Way, M. (2018). Insights into
Kinesin-1 Activation from the Crystal Structure of KLC2 Bound to JIP3. Structure 26, 1486-1498.

Coy, D.L., Hancock, W.O., Wagenbach, M., and Howard, J. (1999). Kinesin's tail domain is an inhibitory regulator
of the motor domain. Nat. Cell Biol. 1, 288-292. DeBerg, H.A., Blehm, B.H., Sheung, J., Thompson, A.R., Bookwalter, C.S., Torabi, S.F., Schroer, T.A., Berger, C.L. Lu, Y., Trybus, K.M., et al. (2013). Motor domain phosphorylation modulates kinesin-1 transport. J. Biol. Chem

DeBoer, S.R., You, Y.M., Szodorai, A., Kaminska, A., Pigino, G., Nwabuisi, E., Wang, B., Estrada-Hernandez, homodimers. Biochemistry 47, 4535-4543. Dent, E.W., Gupton, S.L., and Gertler, F.B.
Cold Spring Harb. Perspect. Biol. 3, 1-39.
Dietrich, K.A., Sindelar, C. V., Brewer, P.D., Downing, K.H., Cremo, C.R., and Rice, S.E. (2008). The kinesin-1

Dorner, C., Ullrich, A., Häring, H.U., and Lammers, R. (1999). The kinesin-like motor protein KIF1C occurs

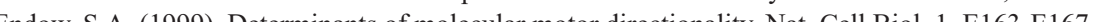

Fields, S., and Song, O.K. (1989). A novel genetic system to detect protein-protein interactions. Nature 340, 245-246. Friedman, D.S., and Vale, R.D. (1999). Single-molecule analysis of kinesin motility reveals regulation by the cargoFriedman, D.S., and Vale, R.D. (1999). Sing-e-n.
binding tail domain. Nat. Cell Biol. 1, 293-297. Fu, M., and Holzbaur, E.L.F. (2014). Integrated regulation of motor-driven organelle transport by scaffolding proteins. Trends Cell Biol. 24, 564-574. Fu, M., Nirschl, J.J., and Holzbaur, E.L.F. (2014). LC3 Binding to the scaffolding protein jip1 regulates processive
dynein-driven transport of autophagosomes. Dev. Cell 29, 577-590. Furuta, K., Furuta, A., and Toyoshima, Y.Y. (2017). Measuring collective transport by defined numbers of processive and nonprocssive K, a

Gan, K.J., Akram, A., Blasius, T.L., Ramser, E.M., Budaitis, B.G., Gabrych, D.R., Verhey, K.J., and Silverman, M.A. (2020). Gsk 33 impairs kifla transport in a cellular model of alzheimer's disease but does not regulate moto

Ghiretti, A.E., Thies, E., Tokito, M.K., Lin, T., Ostap, E.M., Kneussel, M., and Holzbaur, E.L.F. (2016). Activity-
dependent regulation of distinct transport and cytoskeletal remodeling functions of the dendritic kinesin KIF21B. Neuron $92,857-872$.

Grabham, P.W., Seale, G.E., Bennecib, M., Goldberg, D.J., and Vallee, R.B. (2007). Cytoplasmic dynein and LIS1 are
required formicrotubule advance during growth coneremodeling and fast axonal outgrowth. J. Neurosci. 27,5823-5834 Gross, S.P., Vershinin, M., and Shubeita, G.T.T. (2007). Cargo transport: Two motors are sometimes better than one. Curr. Biol. 17, 478-486.

Guardia, C.M., Farías, G.G., Jia, R., Pu, J., and Bonifacino, J.S. (2016). BORC functions upstream of kinesins 1 Guillud, L. Wong R a d Hit phosphorylation: A molecular model of kinesin-cargo release. Nat. Cell Biol. 10, 19-29

Hammond, J.W., Cai, D., Blasius, T.L., Li, Z., Jiang, Y., Jih, G.T., Meyhofer, E., and Verhey, K.J. (2009). Mammalian 7, e1000072.

Hammond, J.W., Blasius, T.L., Soppina, V., Cai, D., and Verhey, K.J. (2010). Autoinhibition of the kinesin-2 motor Han, B., Zhou, R., Xia, C., and Zhuang, X. (2017). Structural organization of the actin-spectrin-based membrane

Hancock, W.O. (2014). Bidirectional cargo transport: Moving beyond tug of war. Nat. Rev. Mol. Cell Biol. 15, 615-628 Hirokawa, N., and Takemura, R. (2005). Molecular motors and mechanisms of directional transport in neurons. Nat.
Rev. Neurosci. 6, 201-214

Hirokawa, N., Noda, Y., Tanaka, Y., and Niwa, S. (2009). Kinesin superfamily motor proteins and intracellular

Hirokawa, N., Niwa, S., and Tanaka, Y. (2010). Molecular motors in neurons: Transport mechanisms and roles in

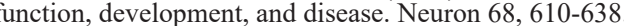

Hoerndli, F.J., Wang, R., Mellem, J.E., Kallarackal, A., Brockie, P.J., Thacker, C., Madsen, D.M., and Maricq, A. V.
(2015). Neuronal activity and CaMKII regulate kinesin-mediated transport of synaptic AMPARs. Neuron 86, 457-474 Hoogenraad, C.C., Wulf, P., Schiefermeier, N., Stepanova, T., Galjart, N., Small, J.V., Grosveld, F., De Zeeuw,
C.II, and Akhmanova, A. (2003). Bicaudal D induces selective dynein-mediated microtubule minus end-directed C.I., and Akhmanova, A. (2003). Bicaudal D induces selective dynein-mediated microtubule minus end-directed
transport. EMBO J. 22, 6004-6015. Hotulainen, P., and Hoogenraad, C.C. (2010). Actin in dendritic spines: Connecting dynamics to function. J. Cell
Biol. 189, 619-629.

Howard, J., and Hyman, A.A. (2009). Growth, fluctuation and switching at microtubule plus ends. Nat. Rev. Mol,
Cell Biol. 10, 569-574. Ichinose, S., Ogawa, T., and Hirokawa, N. (2015). Mechanism of activity-dependent cargo loading via the

chinose, S., Ogawa, T., Jiang, X., and Hirokawa, N. (2019). The spatiotemporal construction of the axon initial segment via KIF3/KAP3/TRIM46 transport under MARK2 signaling. Cell Rep. 28, 2413-2426.

Jacobson, C., Schnapp, B., and Banker, G.A. (2006). A change in the selective translocation of the kinesin-1 moto

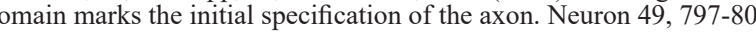

Janke, C. (2014). The tubulin code: Molecular components, readout mechanisms, functions. J. Cell Biol. 206, 461-472 Jenkins, B., Decker, H., Bentley, M., Luisi, J., and Banker, G. (2012). A novel split kinesin assay identifies motor
proteins that interact with distinct vesicle populations. J Cell Biol. 198, 749-761

Jones, S.L., Korobova, F., and Svitkina, T. (2014). Axon initial segment cytoskeleton comprises a multiprotein
submembranous coat containing sparse actin filaments. J. Cell Biol. 205, 67-81. Kaan H. Y Hackney D.D and Kozielski, F. (2011). The structure of the kinesin-1

motor-tail complex reveals the 
Kamei, T., Kakuta, S., and Higuchi, H. (2005). Biased binding of single molecules and continuous movement of

Kapitein, L.C., and Hoogenraad, C.C. (2015). Building the neuronal microtubule cytoskeleton. Neuron 87, 492-506 Kato, Y., Miyakawa, T., and Tanokura, M. (2018). Overview of the mechanism of cytoskeletal motors based on
structure. Biophys. Rev. 10,571-581.

Kaul, N., Soppina, V., and Verhey, K.J. (2014). Effects of $\alpha$-tubulin K40 acetylation and detyrosination on kinesin-1
motility in a purified system. Biophys. J.106, 2636-2 643 . 2636-2643.

Kevenaar, J.T., Bianchi, S., Van Spronsen, M., Olieric, N., Lipka, J., Frias, C.P., Mikhaylova, M., Harterink, M. Keijzer, N., Wulf, P.S., et al. (2016). Kinesin-binding protein controls microtubule dynamics and cargo traffickin by regulating kinesin motor activity. Curr. Biol. 26, 849-861.
Kevin Pfister, K., isher, E.M.C., Gibbons, I.R., Hays, T.S., Holzbaur, E.L.F., Richard McIntosh, J., Porter, M.E.,
Schroer, T.A., Vaughan, K.T., Witman, G.B., et al.(2005).Cytoplasmic dynein nomenclature. J. Cell Biol. 171,411-413. Kneussel, M., and Wagner, W. (2013). Myosin motors at neuronal synapses: Drivers of membrane transport an

Köhler, D., Ruff, C., Meyhöfer, E., and Bähler, M. (2003). Different degrees of lever arm rotation control myosin Lawrence, C.J., Dawe, R.K., Christie, K.R., Cleveland, D.W., Dawson, S.C., Endow, S.A., Goldstein, L.S.B., Lipka, J., Kapitein, L.C., Jaworski, J., and Hoogenraad, C.C. (2016). Microtubule-binding protein doublecortin-like Lo, K.Y., Kuzmin, A., Unger, S.M., Petersen, J.D., and Silverman, M.A. (2011). KIF1 1 i is the primary anterograd motor protein required for the axonal transport of dense-core vesicles in cultured hippocampal neurons. Neurosci.

Lu, W., and Gelfand, V.I. (2017). Moonlighting motors: Kinesin, dynein, and cell polarity. Trends Cell Biol. 27, 505-514. Lu, Q., Li, J., and Zhang, M. (2014). Cargo recognition and cargo-mediated regulation of unconventional myosins

Luo, L. (2002). Actin cytoskeleton regulation in neuronal morphogenesis and structural plasticity. Annu. Rev. Cell

Maday, S., Wallace, K.E., and Holzbaur, E.L.F. (2012). Autophagosomes initiate distally and mature during transport
toward the cell soma in primary neurons. J. Cell Biol. 196, 407-417. Marx, A., Hoenger, A., and Mandelkow, E. (2009). Structures of kinesin motor proteins. Cell Motil. Cytoskeleton
$66,958-966$. McKenney, R.J., Huynh, W., Tanenbaum, M.E., Bhabha, G., and Vale, R.D. (2014). Activation of cytoplasmic dynein motility by dynactin-cargo adapter complexes. Science $345,337-341$

Miki, H., Setou, M., Kaneshiro, K., and Hirokawa, N. (2001). All kinesin superfamily protein, KIF, genes in mouse
and human PNAS $98,7004-7011$, and human. PNAS $98,7004-701$

Morfini, G. (2002). Glycogen synthase kinase 3 phosphorylates kinesin light chains and negatively regulates

Niwa, S., Tanaka, Y., and Hirokawa, N. (2008). KIF1Bß- and KIF1A-mediated axonal transport of presynaptic
regulator Rab3 occurs in a GTP-dependent manner through DENN/MADD. Nat. Cell Biol. 10, 1269-1279.

Niwa, S., Lipton, D.M., Morikawa, M., Zhao, C., Hirokawa, N., Lu, H., and Shen, K. (2016). Autoinhibition of a Nogales, E., and Wang, H.W. (2006). Structural intermediates in microtubule assembly and disassembly: How and
why? Curr. Opin. Cell Biol. 18, 179-184.

Okada, Y., Yamazaki, H., Sekine-Aizawa, Y., and Hirokawa, N. (1995). The neuron-specific kinesin superfamily protein
KIF1 A is a unique monomeric motor for anterograde axonal transport of synaptic vesicle precursors. Cell 81, 769-780 Okada, Y., Higuchi, H., and Hirokawa, N. (2003). Processivity of the single-headed kinesin KIF1A through bindin

Park, J.H., and Roll-Mecak, A. (2018). The tubulin code in neuronal polarity. Curr. Opin. Neurobiol. 51, 95-102. Pollard, T.D., and Borisy, G.G. (2003). Cellular motility driven by assembly and disassembly of actin filaments
Cell $112,453-465$.

Purcell, T.J., Morris, C., Spudich, J.A., and Sweeney, H.L. (2002). Role of the lever arm in the processive stepping
of myosin V. PNAS $99,1459-14164$. Quyen Nguyen, T., Aumont-Nicaise, M., Andreani, J., Velours, C., Chenon, M., Vilela, F., Geneste, C., Varela, P.F., Quyen Nguyen, T., Aumont-Nicaise, M., Andreani, J., Velours, C., Chenon, M., Vilela, F., Geneste, C., Varela, P.F.,
Llinas, P., and Ménétrey, J. (2018). Characterization of the binding mode of JNK-interacting protein 1 (JIP1) to
kinesin-light chain 1 (KLC1) J. Biol. Chem. 293, 13946-13960. Ren, J., Wang, S., Chen, H., Wang, W., Huo, L., and Feng, W. (2018). Coiled-coil 1-mediated fastening of the neck and motor domains for kinesin-3 autoinhibition. PNAS 115, E11933-E11942

Robert-Paganin, J., Pylypenko, O., Kikuti, C., Sweeney, H.L., and Houdusse, A. (2020). Force generation by myosin motors: A structural perpective. Chem. Rev. 120, 5-35.

Roberts, A.J., Kon, T., Knight, P.J., Sutoh, K., and Burgess, S.A. (2013). Functions and mechanics of dynein motor
proteins. Nat. Rev. Mol. Cell Biol. 14, 713-726.

Roossien, D.H., Lamoureux, P., and Miller, K.E. (2014). Cytoplasmic dynein pushes the cytoskeletal meshwork
forward during axonal elongation. J. Cell Sci. 127, 3593-3602. forward during axonal elongation. J. Cell Sci. 127, 3593-3602.
Satake, T., Otsuki, K., Banba, Y., Suenaga, J., Hirano, H., Yamanaka, Y., Ohno, S., and Hirai, S.I. (2013). The interaction of Kinesin-1 with its adaptor protein JIP1 can be regulated via proteins binding to the JIP1-PTB domain.

Schelski, M., and Bradke, F. (2017). Neuronal polarization: From spatiotemporal signaling to cytoskeletal dynamics.

Schimert, K.I., Budaitis, B.G., Reinemann, D.N., Lang, M.J., and Verhey, K.J. (2019). Intracellular cargo transport
by single-headed kinesin motors. PNAS $116,6152-6161$ Scholey, J.M. (2013). Kinesin-2: A family of heterotrimeric and homodimeric motors with diverse intracellular
transport functions. Annu. Rev. Cell Dev. Biol. 29, 443-469. Schroer, T.A. (2004). Dynactin. Annu. Rev. Cell Dev. Biol. 20, 759-779.

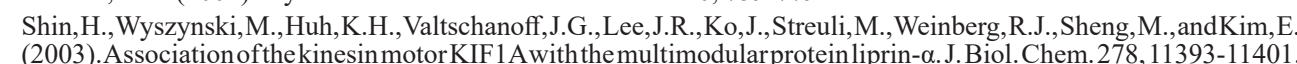
(2003).Association of the kinesinmotor KIF1 A with the multimodularprotein liprin- - .J. Biol.Chem. 278, 11393-11401, Siddiqui, N., Zwetsloot, A.J., Bachmann, A., Roth, D., Hussain, H., Brandt, J., Kaverina, I., and Straube, A. (2019).
PTPN21 and Hook3 relieve KIF1C autoinhibition and activate intracellular transport. Nat. Commun. 10, 1-16. Sirajuddin, M., Rice, L.M., and Vale, R.D. (2014). Regulation of microtubule motors by tubulin isotypes and

Soppina, V., Norris, S.R., Dizaji, A.S., Kortus, M., Veatch, S., Peckham, M., and Verhey, K.J. (2014). Dimerization
of mammalian kinesin-3 motors results in usuperprocessive motion PNAS $111,5562-5567$.

Rev. Neurosci. 15, 683-695.

Stucchi, R., Plucińska, G., Hummel, J.J.A., Zahavi, E.E., Guerra San Juan, I., Klykov, O., Scheltema, R.A., Altelaar, A.F.M., and Hoogenraad, C.C. (2018). Regulation of KIF1A-driven dense core vesicle transport:
DCV binding and Liprin- $\alpha$ /TANC2 recruits DCVs to postsynaptic sites. Cell Rep. 24, 685-700. Tempes, A., Weslawski, J., Brzozowska, A., and Jaworski, J. (2020). Role of dynein-dynactin complex, kinesins,
motor adaptors, and their phosphorylation in dendritogenesis. J. Neurochem. 155, 10-28.

van der Vaart, B., van Riel, W.E., Doodhi, H., Kevenaar, J.T., Katrukha, E.A., Gumy, L., Bouchet, B.P., Grigoriev I., Spangler, S.A., Yu, K. Lou, et al. (2013). CFEOM1-associated kinesin KIF21A is a cortical microtubule growth

Vagnoni, A., Rodriguez, L., Manser, C., De Vos, K.J., and Miller, C.C.J. (2011). Phosphorylation of kinesin light
chain 1 at serine 460 modulates binding and trafficking of calsyntenin-1.J Cell Sci. 124, $1032-1042$ (1996). Direct observation of single kinesin molecules moving along microtubules. Lett. to Nat. 380, 451-453.

Verhey, K.J., and Hammond, J.W. (2009). Traffic control: Regulation of kinesin motors. Nat. Rev. Mol. Cell Biol.

Verhey, K.J., Meyer, D., Deehan, R., Blenis, J., Schnapp, B.J., Rapoport, T.A., and Margolis, B. (2001). Cargo of
kinesin identified as JIP scaffolding proteins and associated signaling molecules. J. Cell Biol. 152, 959-970. Wang, X., and Schwarz, T.L. (2009). The mechanism of $\mathrm{Ca}^{2+}$-dependent regulation of kinesin-mediated mitochondrial

Wang, W., Cao, L., Wang, C., Gigant, B., and Knossow, M. (2015). Kinesin, 30 years later: Recent insights from Watanabe, K., Al-Bassam, S., Miyazaki, Y., Wandless, T.J., Webster, P., and Arnold, D.B. (2012). Networks of polarized actin filaments in the axon initial segment provide a mechanism for sorting axonal and dendritic proteins. Cell Rep. 2, 1546-1553.

Wells, A.L., Lin, A.W., Chen, L.Q., Safer, D., Cain, S.M., Hasson, T., Carragher, B.O., Milligan, R.A., and Sweeney, Welte, M.A., Gross, S.P., Postner, M., Block, S.M., and Wieschaus, E.F. (1998). Developmental regulation of vesicle

Wieczorek, M., Bechstedt, S., Chaaban, S., and Brouhard, G.J. (2015). Microtubule-associated proteins control the

Curr. Opin. Cell Biol. 17, 82-88.

Wozniak, M.J., Milner, R., and Allan, V. (2004). N-terminal kinesins: Many and various. Traffic 5, 400-410.

Wozniak, M.J., Melzer, M., Dorner, C., Haring, H.U., and Lammers, R. (2005). The novel protein KBP regulates mitochondria localization by interaction with a kinesin-like protein. BMC Cell Biol. 6, 1-15.

Xie, P. (2010). Mechanism of processive movement of monomeric and dimeric kinesin molecules. Int. J. Biol. Sci. $\mathrm{Xu}, \mathrm{K}$., Zhong, G., and Zhuang, X. (2013). Actin, spectrin, and associated proteins form a periodic cytoskeletal structure in axons. Science $339,452-456$.

Yang, R. Bentley, M., Huang, C.-F., and Banker, G. (2016). Analyzing kinesin motor domain translocation in
cultured hippocampal neurons. Methods Cell Biol. 131, 217-232

Yau, K.W., Schätzle, P., Tortosa, E., Pagès, S., Holtmaat, A., Kapitein, L.C., and Hoogenraad, C.C. (2016). Dendrites In vitro and In vivo contain microtubules of opposite polarity and axon formation correlates with uniform plus-end-
out microtubule orientation. J. Neurosci. 36, 1071-1085.

Yildiz,A., Tomishige, M., Vale, R.D., and Selvin,P.R. (2004). Kinesinwalkshand-over-hand. Science (80) 303, 676-678 duty ratio mechanism unlike that of two-headed kinesin. Bioch kinesin derivatives move by a nonprocessive, low- 


\section{The mechanism of kinesin inhibition by kinesin-binding protein}

Joseph Atherton ${ }^{1,2}$, Jessica J. A. Hummel ${ }^{3}$, Natacha Olieric ${ }^{4}$, Julia Locke ${ }^{2,5}$, Alejandro Peña ${ }^{2,6}$, Steven S. Rosenfeld ${ }^{7}$, Michel O. Steinmetz ${ }^{4,8}$, Casper C. Hoogenraad ${ }^{3}$, and Carolyn A. Moores ${ }^{2}$

eLife (2020);9:e61481

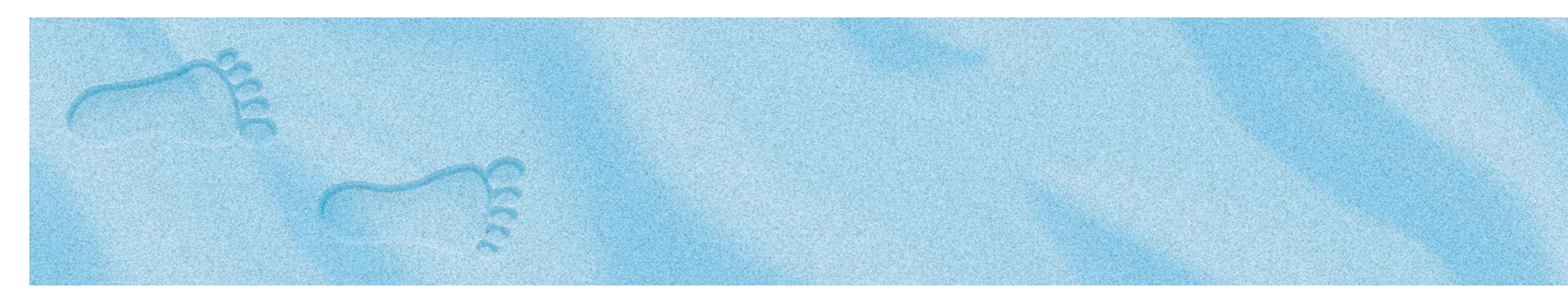

${ }^{1}$ Randall Centre for Cell and Molecular Biophysics, King's College, London, United Kingdom.

${ }^{2}$ Institute of Structural and Molecular Biology, Birkbeck, University of London, London, United Kingdom.

${ }^{3}$ Cell Biology, Neurobiology and Biophysics, Department of Biology, Faculty of Science, Utrecht University, Utrecht, the Netherlands.

${ }_{4}^{4}$ aborecht University, Utrecht, he Nethe ${ }^{4}$ Laboratory of Biomolecular Research, Division of Biology and Chemistry, Paul Scherre Institut, Villigen PSI, Switzerland.

${ }_{5}^{5}$ Current address: Macromolecular Machines Laboratory, The Francis Crick Institute, London, United Kingdom.

${ }^{6}$ Current address: Department of In Silico Drug Discovery, Pharmidex Pharmaceuticals, Hatfield, United Kingdom.

${ }^{7}$ Department of Cancer Biology, Mayo Clinic, Jacksonville, FL, USA.

${ }^{8}$ University of Basel, Biozentrum, CH-4056 Basel, Switzerland. 


\section{ABSTRACT}

Subcellular compartmentalisation is necessary for eukaryotic cell function. Spatial and temporal regulation of kinesin activity is essential for building these local environments via control of intracellular cargo distribution. Kinesin-binding protein (KBP) interacts with a subset of kinesins via their motor domains, inhibits their microtubule (MT) attachment, and blocks their cellular function. However, its mechanisms of inhibition and selectivity have been unclear. Here we use cryo-electron microscopy to reveal the structure of KBP and of a KBP-kinesin motor domain complex. KBP is a tetratricopeptide repeat-containing, right-handed $\alpha$-solenoid that sequesters the kinesin motor domain's tubulin-binding surface, structurally distorting the motor domain and sterically blocking its MT attachment. KBP uses its $\alpha$-solenoid concave face and edge loops to bind the kinesin motor domain, and selected structure-guided mutations disrupt KBP inhibition of kinesin transport in cells. The KBPinteracting motor domain surface contains motifs exclusively conserved in KBP-interacting kinesins, suggesting a basis for kinesin selectivity.

\section{INTRODUCTION}

Kinesins are a superfamily of microtubule (MT)-based molecular motors that play importan roles in cellular functions such as mitosis, cell motility, and intracellular transport (Vale, 2003; Hirokawa et al, 2009; Klinman and Holzbaur, 2018). Kinesins are categorised into 14 sub-classes (kinesin-1 to kinesin-14 [Lawrence et al., 2004]) by motor domain conservation and within these sub-classes individual family members (a total of 45 'KIF' or 'Kif' genes in humans and mice respectively) have a wide range of functional characteristics and biological roles (Vale, 2003; Miki et al., 2001). Dysfunction of kinesin family members has been implicated in a number of pathological conditions (Hirokawa et al., 2010; Mandelkow and Mandelkow, 2002). The kinesin motor domain is the MT-binding engine that drives these activities, converting the chemical energy of ATP binding and hydrolysis into mechanical force. While these mechanical forces are classically used to generate motility in transport kinesins, some kinesin family members drive MT organisation or depolymerisation of MTs.

Kinesins are highly regulated in order to prevent both waste of ATP and to spatially and temporally control kinesin function. This is particularly important in highly polarised and compartmentalised cells such as neurons. Kinesin regulation via inhibition of their motor domains can occur through a number of mechanisms that limit ATPase activity and/o block track binding - these include intramolecular inhibition by kinesin tail domains, posttranslational modification of the motor, or through interactions with regulatory binding partners. Recently, it has been demonstrated that a subset of kinesin superfamily members, including kinesin-2s, $-3 \mathrm{~s},-8 \mathrm{~s}$, and $-12 \mathrm{~s}$, are sequestered by kinesin-binding protein (KBP; KIF1BP, KIAA1279), which inhibits MT track attachment by their motor domains and, thus, blocks their MT-related functions (Wozniak et al, 2005; Kevenaar et al, 2016; Alves et al., 2010).

KBP is expressed in multiple human tissues including brain and heart (Wozniak et al., 2005). Mutations in the KBP have been identified as causing autosomal recessive GoldbergShprintzen syndrome (GOSHS) (Brooks et al., 2005; Dafsari et al., 2015; Valence et al., 2013; Salehpour et al., 2017), which presents as congenital facial dysmorphia, nervous system pathology, and dysfunction and heart defects (Tanaka et al., 1993). In addition, KBP gene copy number has been recently reported as predictive in paediatric neuroblastoma prognosis, prompting its suggestion as a drug target (Suo et al., 2018). KBP was originally identified as a kinesin-3-binding partner that modulated its mitochondrial transport function (Wozniak et al.,2005); however, KBP has since been shown to interact with a subset of other kinesin amily mets growth, and cargo distribution (Alves et al., 2010; Lyons et al., 2008; Drévillon et al., 2013; Drerup et al., 2016; Chang et al., 2019; Hirst et al., 2017)
We do not currently know what the structure of KBP is, nor understand the mechanism of KBP-kinesin inhibition. It is also completely unknown how KBP differentiates between particular kinesin family members. KBP is a $72 \mathrm{kDa}$ protein, is predicted to contain severa tetratricopeptide repeats (TPRs), and to be mainly $\alpha$-helical in secondary structure content structures of KBP alone and of KBP bound to mitotic kinesin KIF15 (a $110 \mathrm{kDa}$ complex). We show that KBP is a TPR-containing, righthanded $\alpha$-solenoid protein composed of nine antiparallel $\alpha$-helix pairs interrupted by a linke region. We also show that KBP's concave face binds KIF 15 via its MT-binding elements an induces a large displacement of the kinesin $\alpha 4$ helix, sterically inhibiting MT association. Finally, we show that KBPs kinesin selectivity is associated with specific kinesin sequences spread across the interaction surface.

\section{RESULTS}

PR-containing, right-handed $\alpha$-solenoid

The 3D structure of the $\sim 72 \mathrm{kDa} \mathrm{KBP}$ at $4.6 \AA$ resolution (Fig 1; Fig 1-S1A,B) was determined using cryo-EM data collected using a Volta phase plate (VPP), and an atomic model was calculated (see Materials and methods Table 1). Our structure revealed that KBP is a right-handed $\alpha$-solenoid protein (Fig 1A,B; Fig 1-S1C-E). Nine pairs of anti-parallel $\alpha$-helices ( $\alpha \mathrm{HP} 1$ [ $\alpha$-helical pair 1] to $\alpha \mathrm{HP9})$ are broken by a single 'linker $\alpha$-helix' $(\mathrm{L} \alpha \mathrm{H})$ and 'linker loop' (LL) in the centre of the fold separating KBP into N-terminal and C-termina subdomains (Fig 1; Fig 1-S1C-E; Fig 1-S2). The four predicted TPR motifs contribute exclusively to $\alpha$-helical pairs in the N-terminal subdomain (Fig 1A,D,E).

The supercoiling $\alpha$-helical pairs form concave and convex faces linked by short and long loops that constitute the two edges of the $\alpha$-solenoid (Fig 1-S1C-E; Fig 1-S2). In contras to the shorter loops, the longer loops (more than seven residues) tend to be partially disordered, show low sequence homology between KBP orthologues in different species, and are mainly found in the N-terminal subdomain (e.g. L2, L6, and L10; Fig 1-S1D,E; Fig 1-S2). The LL is the longest (62 residues) and is thus unique in the KBP structure because it is reasonably conserved and mainly ordered, with visible corresponding density clearly bridging the $\mathrm{N}$ - and C-terminal subdomains(Fig 1A,B; Fig 1-S1D,E· Fig 1-S2; Fig 1-S3). Despite this clear ordered was not modelled due to low homology to available structures and a lack of consensus in secondary structure prediction (see Materials and methods). In spite of this lack of consensus, density in this region suggests that part of this loop may form further $\alpha$-helica structures. Other TPR-containing $\alpha$-solenoid proteins form important regulatory interaction in numerous contexts, and the structure we describe is indicative of similar properties for KBP.

KBP conformationally adapts to bind KIF15's motor domain using both

To elucidate the mechanism of kinesin inhibition by KBP, we determined the structure of KBP in complex with the human KIF15 (kinesin-12) motor domain (KIF15_MD, 1-375). This construct, which has six of its eight cysteine residues mutated to serine (C5S, C50S C162S, C294S, C314S, and C346S) and two additional cysteines were inserted (S250C and $\mathrm{G} 375 \mathrm{C}$ ), has comparable steady-state ATPase activity to previously published report (Klejnot et al., 2014; Fig 2-S1A) and we refer to it as KIF15 MD6S. The overall resolutio (Fig 2-S1B,C). We built a model of the complex via flexible fitting using our KBP model and the KIF15_MD crystal structure (Fig 2A,B; Table 1, Materials and methods). The complex is arranged such that KIF15_MD6S sits in the concave face of the KBP $\alpha$-solenoid, analogous to a baseball enclosed in a baseball glove. The kinesin MD is positioned centrally between the $\mathrm{N}$ - and $\mathrm{C}$-terminal subdomains and contacts the $\mathrm{KBP}$ concave face and loops at the $\alpha$-solenoid edges. 


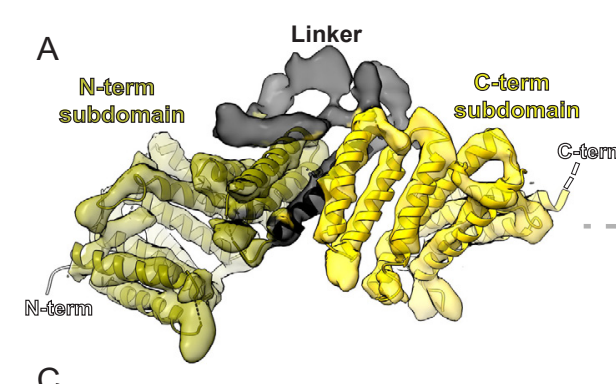

B sulbermm
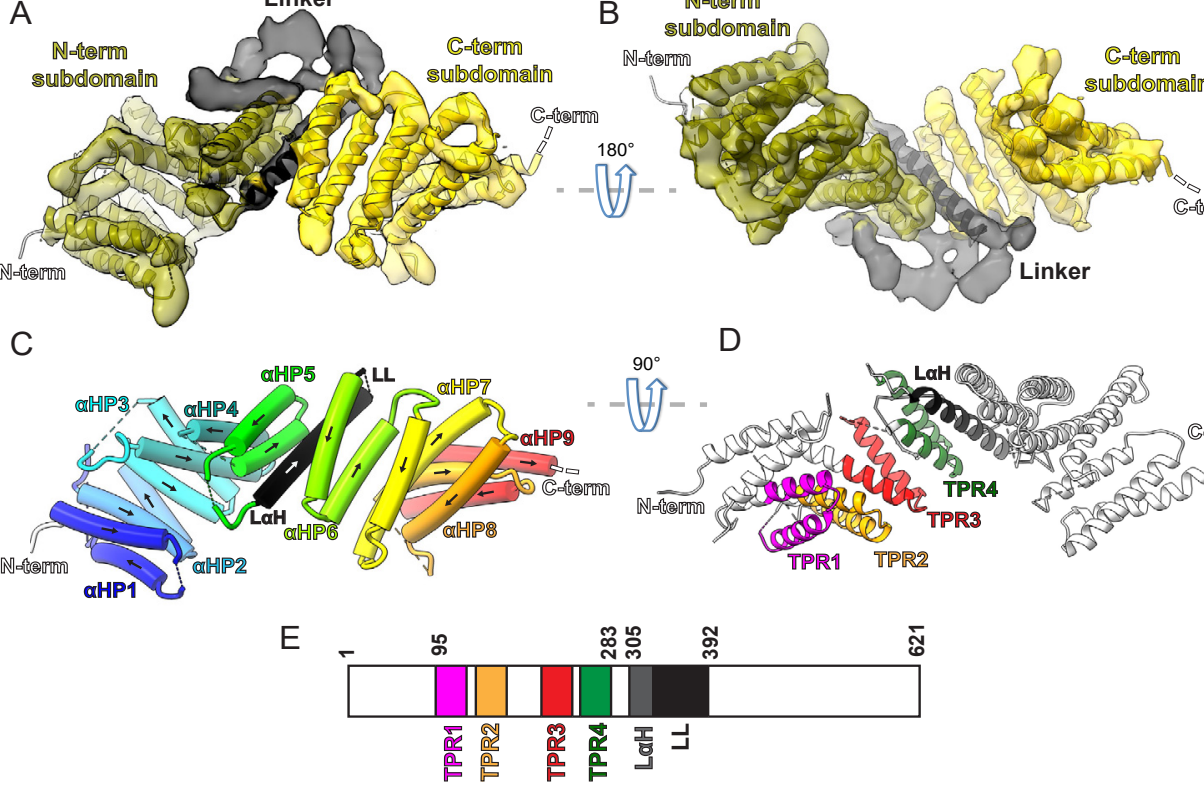

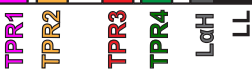

NNotierm subdomalln

Gatarm subdomath

Figure 1. Kinesin-binding protein (KBP) is a tetratricopeptide repeat(TPR)-containing right-handed $\alpha$-solenoid (A) Model of KBP (ribbon representation) displayed in experimental cryo-electron microscopy density. The $\mathrm{N}$-terminal (olive) and C-terminal (gold) subdomains are separated by a linker region (black). Semi-transparen density is coloured regionally as per the fitted model. The $\mathrm{N}$ - and $\mathrm{C}$-termini are shown, with a dotted line representing the disordered C-terminus (not modelled). The linker loop (LL) region was not modelled but its density is shown in semi-transparent black. (B) The same as panel a, but rotated $180^{\circ}$ around the axis indicated. (C) The same view as in panel a, but with the density removed and $\alpha$-helices displayed as pipes with their directionality indicated by arrows (LaH) and LL (dotted line). (D) Ribbon representato of KBP showing the four TPR motifs and the LoH $\alpha$-helix according to the labels. View related to panel c, by a $90^{\circ}$ rotation around the indicated axis. (E) Schematic of the KBP showing the position of the TPR motifs between residue 95 and 283 of the N-terminal subdomain and position of the linker region ( $\mathrm{L} \alpha \mathrm{H}$ and $\mathrm{LL}$ ) between residues 305 and 392.

When the structure of KBP-alone is superimposed onto KBP in the KBP-KIF15 MD6S complex, it is clear that KBP undergoes a conformational change in the presence of its kinesin motor domain-binding partner, with the largest differences resulting from an unfurling motion of its N-terminal subdomain (Fig 2C,D; Video 1). The KBP-alone mode is incompatible with KIF15 MD6S binding, due to clashes with L14 in the C-terminal subdomain and $\alpha \mathrm{HP} 3 \mathrm{a}, \alpha \mathrm{HP} \overline{4} \mathrm{a}$ and L8 in the N-terminal subdomain. The conformational changes in KBP upon KIF15 MD6S binding relieve these clashes in the complex (Video 1). To establish whether the KBP-KIF15 MD6S mode of interaction applied to other kinesins, we also collected data of the complex formed by KBP with the motor domain of the human kinesin-3 KIF1A (KIF1A_MD). Two-dimensional classification of these image revealed a number of classes with an extra-density corresponding to the size of a kinesin motor domain bound to the concave face of KBP, consistent with what was observed in the KBP-KIF15_MD6S dataset (Fig 2-S1C). However, in contrast to the KBP-KIF15 MD6S sample, these KBP-KIF1A_MD 2D classes provided only limited views of the complex (Fig 2-S1C,D), such that a reliable 3D structure could not be calculated. Intriguingly, in addition, the extra kinesin density in the 2D classes appeared to have a somewhat flexible position relative to KBP. However, these data did allow us to confirm that indeed KIF1A MD also interacts with KBP on its concave face in the same way as KIF15_MD6S and suggests a common mechanism of kinesin inhibition by KBP.
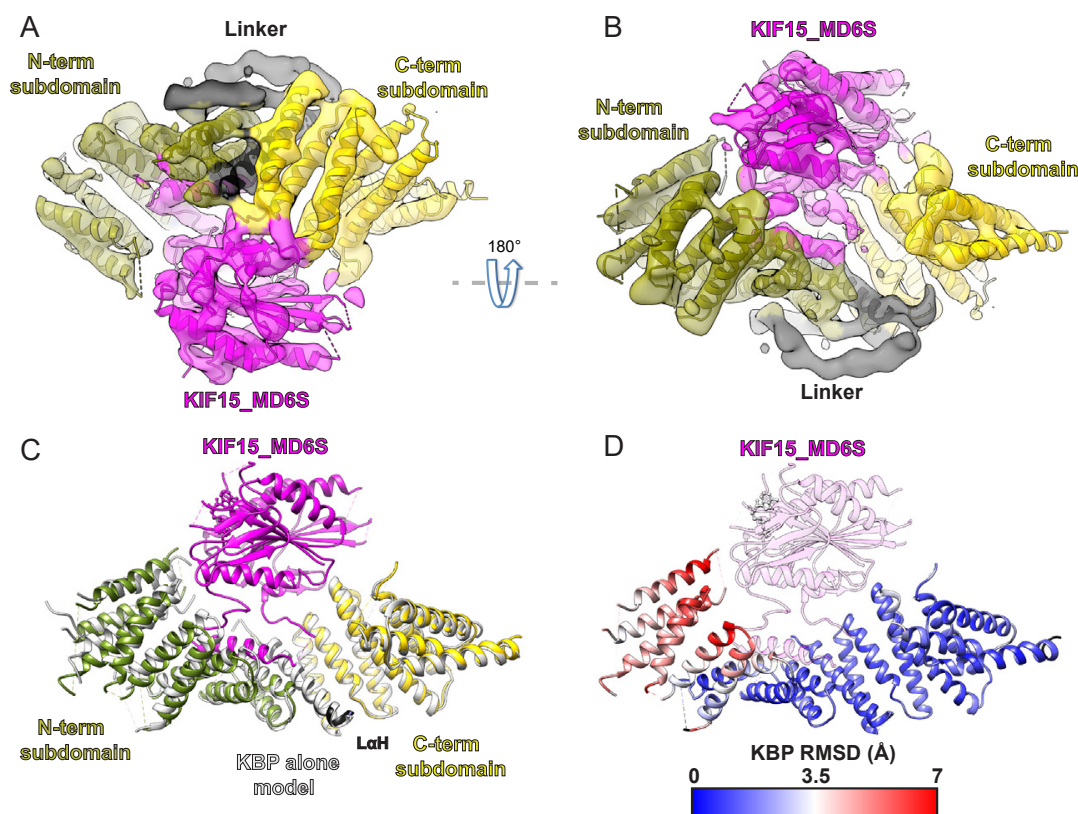

D KIF15_MDES

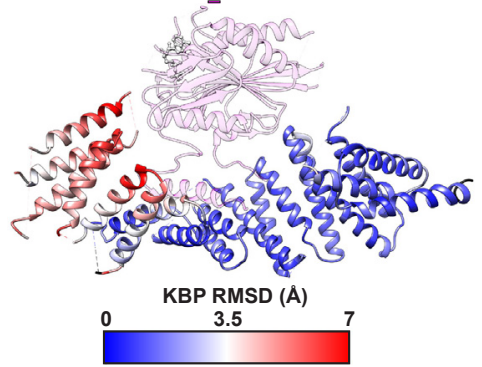

Figure 2. Kinesin-binding protein (KBP) conformationally adapts to bind KIF15's motor domain via both

(A) Model of the KBP-KIF15_MD6S complex (ribbon representation) displayed in experimental cryo-electron microscopy density. The N-terminal (olive) and C-terminal (gold) subdomains and the linker helix (black) are shown in KB, wile kine $180^{\circ}$ around the axis indicated. (C) The KBP-alone model (light grey ribbons) was superimposed on the KBPKIF15 MD6S model (opaque ribbons) using Chimera's matchmaker (Pettersen et al., 2004). Colouring and view as in panel B. (D) RMSD in $\AA$ for KBP comparing KBP-KIF15 MD6S and superimposed KBP-alone models as in panel c, shown on KBP from the KBP-KIF15 MD6S model. Parts of the KBP model coloured black are disordered missing in the KBP alone model. The KIF15_MD6S is shown in transparent magenta.

KIF15_MD6S binds KBP via rearrangement of its tubulin-binding subdomain We examined the effect of KBP binding on the conformation of KIF15_MD6S. Kinesi motor domains can be structurally divided into three distinct subdomains (Shang et al., 2014 Gigant et al., 2013) which undergo coordinated conformational changes during the MT-based kinesin ATPase cycle. MT binding stabilises the tubulin-binding subdomain of the MD while the P-loop and Switch 1/2 subdomains - which contain the conserved nucleotide-coordinating P-loop and Switch 1 and 2 motifs - move relative to each other in response to the nucleotide state of the MD (Shang et al., 2014; Gigant et al., 2013; Atherton et al.,2014). We determined the structure of the MT-bound, AMPPNP state of KIF15 MD6S, which shows that this MD adopts a canonical conformation (Fig 3-S1). Comparison of this conformation with an ADPbound Kif15_MD crystal structure (PDB: 4BN2 Klejnot et al., 2014) illustrates the scale of these MT- and nucleotide-dependent subdomain rearrangements in KIF15, which are similar to those seen in other kinesins MDs (Shang et al., 2014; Atherton et al., 2014; Atherton et al., 2017; Fig 3-S1D,E; Fig 3C,D).

The structure of the KBP-KIF15 MD6S complex revealed that KBP binds the kinesin motor domain via the tubulin-binding subdomain (Fig 3). While the P-loop and Switch 1/2 subdomains of the KIF15 MD crystal structure and associated $\mathrm{Mg}^{2+}$-ADP generally fitted well into density of the KBP-KIF15 MD6S complex, a large portion of the tubulin-binding subdomain did not (Fig 3A; Fig 3-S2A,B). In particular, there is a striking lack of density in the expected position for helix a4 (Fig 3A; Fig 3-S2B). 
Instead, there was a strong density of length and width consistent with helix $\alpha 4$ displaced by $\sim 15 \AA$ into the concave face of KBP, which we modelled as such (Fig 3B,E; Fig 2; Fig 3 -S2B-E). This displacement of helix $\alpha 4$, which lies close to the TPR-repeat region of the $\mathrm{N}$-terminal subdomain of KBP, is accompanied by additional rearrangements of the flanking L11 and L12 in KIF15 MD6S (labelled KL11 and KL12; Fig 3C-E; Fig 3-S2B-E). A number of other TPR-containing $\alpha$-solenoids are known to bind peptide motifs with $\alpha$-helical content within their concave faces (Culurgioni et al., 2011; Wang et al., 2009; Quinaud et al., 2007; Fig 3-S3), and our structure shows that KBP binds helix $\alpha 4$ of KIF15_MD6S in a similar way. The KBP-bound conformation of the KIF15_MD6S tubulin-binding subdomain is also radically different from its MT-bound conformation (Fig 3D,E). The tubulin-binding subdomain forms the majority of the MT-binding surface in the KIF15 MD6S-MT complex (Fig 3D; Fig 3-S1) such that KBP and MTs cannot simultaneously bind KIF15 MD6S due to extensive steric overlap (Fig 3D,E). In summary, KBP sequesters and blocks the MTthat involves significant conformational change within the motor domain.

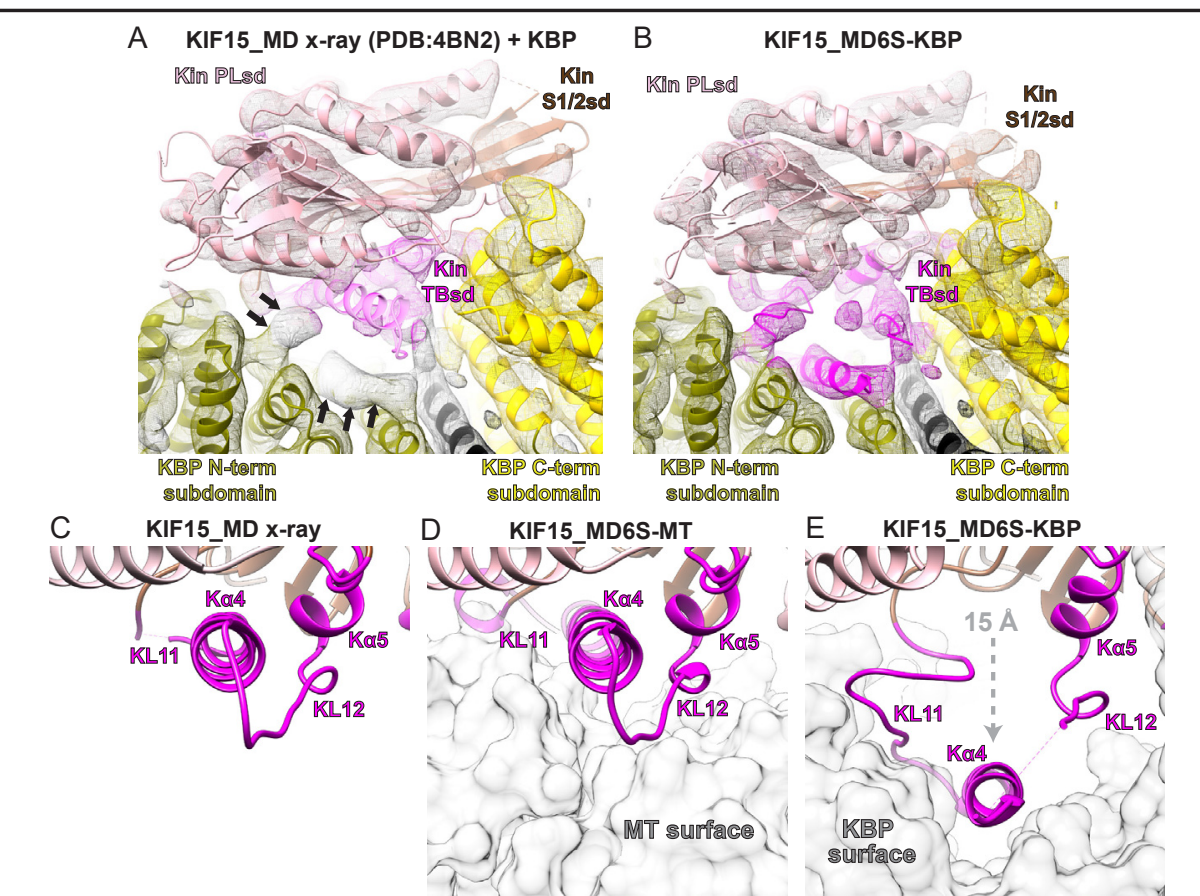

Figure 3. The KIF15 motor domain binds kinesin-binding protein (KBP) via rearrangement of its tubulinbinding subdomain

(A) The crystallographic model of the KIF15_MD alone (PDB: 4BN2 Klejnot et al., 2014) was superimposed on the KIF15 region of the KBP-KIF15_MD6S complex, with the KIF15 part of the KBP-KIF15_MD6S complex model hidden. The KIF15_MD6S Switch 1/2 subdomain (Switch 1/2 subdomain) is coloured sienna, and the P-loo subdomain (Kin-PLsd) is coloured light pink. The TBsd of the KIF15_MD crystallographic model is shown as pale magenta to illustrate poor fit into density. The KBP subdomains are coloured as labelled. Black arrows indicate unaccounted-for cryo-electron microscopy (cryo-EM) density. Individual secondary structure elements in the

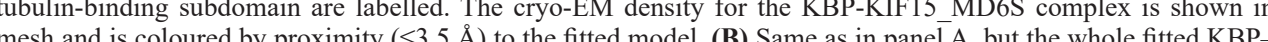
KIF15 MD6S complex model is shown. The KIF15 MD6S tubulin-binding subdomain (TBsd) is now coloured magenta to indicate good fit into density. (C) Zoomed view of just the TBsd (corresponding to the boxed region in Fig 3-S2D), showing just the KIF15 MD-alone crystallographic model. (D) The TBsd in the KIF15 MD6S-MT model, same view as in panel C. The MT is shown in light grey surface representation. (E) The TBsd in the KBP-
KIF15_MD6S model, same view as in panel C. KBP is shown in light grey surface representation and the $\sim 15 \AA$ displacement of helix $\alpha 4$ is indicated by the dashed grey arrow.
KBP binds kinesin motor domains via conserved motifs in the $\alpha$-solenoid edge oops and $\alpha$-helices at the concave face

KBP contacts the KIF15_MD6S both via (1) loops connecting the $\alpha$-solenoid edges and (2) TPR-containing $\alpha$-helices at the concave face (Fig 4; Fig 4-S1; Video 2). At the $\alpha$-solenoid D6S to be involved in closest interaction of these was KBP L12 and L14, which contact both K $\beta 5-K L 8$ and KL12Ka5-KL13 regions of the KIF15 tubulin-binding subdomain (Fig 4). KBP's disordered L lies close to KIF15_MD6S's KL9, while the shorter, ordered L3 and L5 are situated near but not contacting KL11 and Ka6 (Fig 4-S1A,B). KBP's C-terminal L16 and L18 are close enough to KIF15_MD6S that they may interact with the flexible KL12, N-terminus, or necklinker. At the TPR-containing region of the concave face of KBP, $\alpha \mathrm{HP} 4 \mathrm{a}, \alpha \mathrm{HP} 4 \mathrm{~b}$, and $\alpha \mathrm{HP} 5 \mathrm{a}$ contact the K11-Ka4-KL12 region of KIF15 MD6S (Fig 4C,D).

To test the functional significance of this interface, we investigated KBP-kinesin interactions in cells and examined the activities of mutant KBP constructs in which the predicted interacting amino acids within potentially kinesin-contacting loops were substituted for Ala, Gly, or Pro residues (Fig 1-S2; Table 2). Ala-substitutions in the TPR-containing $\alpha$-helices at the KBP concave face were also introduced at particularly inter-species conserve polar residues predicted to interact with the KIF15_MD K11-Ka4-KL12 region (Tyr-213 and Gln-216 in $\alpha \mathrm{HP} 4 \mathrm{a}$, Gln-238 in $\alpha \mathrm{HP} 4 \mathrm{~b}, \mathrm{Thr}-255$ and Gln-258 in $\alpha \mathrm{HP} 5 \mathrm{a}$; Fig 4C, D; Fig 1-S2). All mutant constructs exhibited roughly equivalent expression patterns that were also comparable to WT KBP (Fig 5-S1).

We first used pull-down assays. Mouse Kif15 or Kif1 A constructs consisting of only the motor domain and the first coiled-coil region (Kif15 MDC or Kif1A MDC) were fused to bioGFP and co-expressed with various HA-tagged human KBP constructs in HEK293T cells, followed by pull-down of HA-KBP by the bioGFP-KIF MDC (Kevenaar et al., 2016; Fig 5-S2). Although there are moderate qualitative differences in binding by the two motors, the effects of KBP mutations on motor binding - described in the following - are essentially the same. Ala-substitutions in the TPR-containing $\alpha$-helices at the KBP concave face $(\alpha \mathrm{HP} 4 \mathrm{a}$ and $\alpha \mathrm{HP} 4 \mathrm{~b}$ ), which lie at the heart of the KBP-KIF15 MD6S structural interface, strongly reduced KBP's interaction with both KIF15 MDC and KIF1A MDC. $\alpha$ HP5a mutants had a similar but less pronounced effect (Fig 5-S2B,C). In contrast, mutation of L1, L3, or L 5 in orm directly visualised interactions with KIF15_MD6S in the cryo-EM reconstuction - has no effect on KBP's interaction with either KIF15_MDC or KIF1A_MDC (Fig 5-S2B,C) Mutation of L12 (to some extent) and of L14 (to a greater extent) - which contact both K $\beta 5$ KL8 and KL12-Ka5-KL13 - reduced KBP interaction with KIF15_MDC and KIF1A_MDC (Fig 5-S2B,C). Mutation of L12 + L14 additively disrupted the KBP-motor interaction, consistent with the structural proximity of these two loops in the kinesin-KBP complex. L10 + L12 and L10 + L14 mutants also had weaker interactions with KIF15 MDC/KIF1A MDC (Fig 5-S2B,C), again pointing to the additive contributions of loops in the KBP C-terminal subdomain to kinesin binding. Strikingly, mutation of L18 appears to enhance the interaction between KBP and both KIF15 MDC and KIF1A MDC, suggesting that it may somehow contribute to negative regulation of binding in the context of WT KBP.

We then used a previously described inducible peroxisome translocation assay in COS-7 cells (Kevenaar et al., 2016). In this assay, dimeric mouse Kif15 MDC or Kif1 A MDC constructs with an FRB-tag (Kif15_MDC-FRB or Kif1A_MDC-FR̄B) are expressed together with PEX-mRFP-FKBP, a peroxisome-binding construct, along with the various KBP constructs. Addition of rapalog induces FRB-FKBP heterodimerisation and motordriven peroxisome translocation to the cell periphery, but when the motor is inhibited by KBP, peroxisometranslocationisblocked(Fig 5 A-C) Kinesin-mediated translocationwasme first by quantifying the number of cells in which peroxisome translocation is seen (Fig 5D,E), 


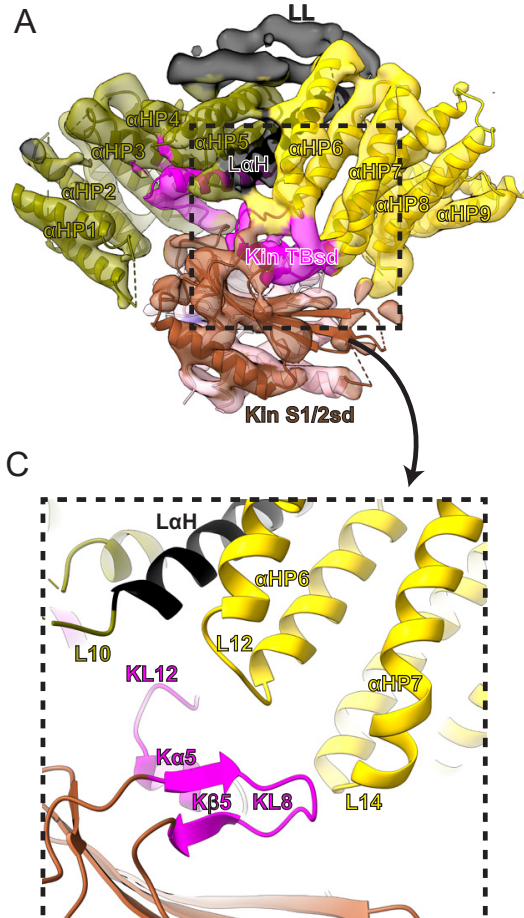

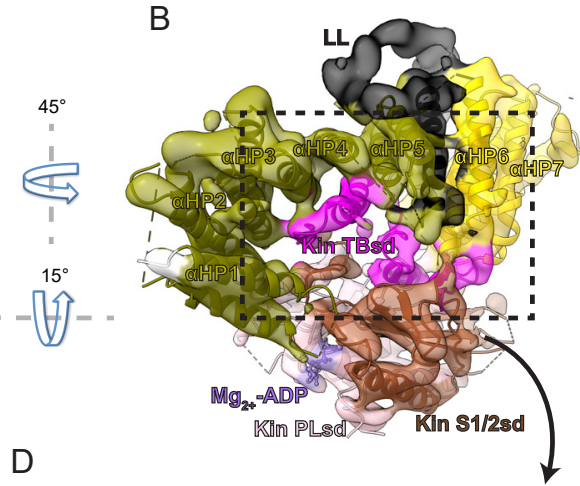

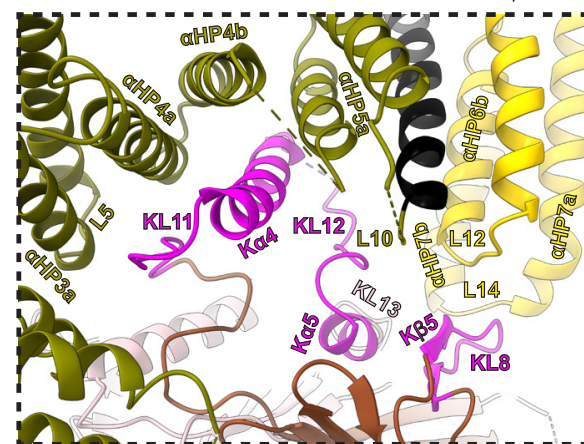

Figure 4. Kinesin-binding protein (KBP) binds kinesin MDs via conserved motifs in the $\alpha$-solenoid edge loops and $\alpha$-helices at the conction

(A) Pseudo-atomic model of the KBP-KIF15_MD6S complex (ribbon representation) displayed in cryo-electron microscopy density, using the same viewpoint as Figure 2a, but with the KIF15_MD6S now coloured by subdomain as in Figure 3. The KIF15_MD6S Switch 1/2 subdomain (Kin S1/2 sd) is coloured sienna, and the P-loop subdomain (Kin-PLsd) is coloured light pink. The KIF15_MD6S tubulin-binding subdomain (TBsd) is coloured magenta. The KBP subdomains are coloured as labelled. The nine helix pairs of KBP are labelled. Semi-transparent density is coloured regionally as per the fitted model and additional density for the linker loop is shown in semi-transpare black. (B) The same as panel A, but rotated $45^{\circ}$ and $15^{\circ}$ respectively around he axes indicated. (C) Zoomed view tements MD6S and KBP secondary structure elements labelled.

and second, by quantifying peroxisome intensities above a threshold value in the cell periphery (Fig 5F,G). Because of observed differences in peroxisome translocation within the time-frame of rapalog treatment, different peroxisome intensity threshold values and peripheral areas were used for KIF1A and KIF15; this is probably due to differences in motor properties (Fig 5-S3).

Intriguingly, while the overall trends in perturbation of KBP inhibition by mutagenesis seen in the pull-down assay are recapitulated in the translocation assay, some differences are also observed. As with the pull-down assay, Ala-substitutions in the TPR containing $\alpha$-helices $\alpha \mathrm{HP} 4 \mathrm{a}$ and $\alpha \mathrm{HP} 4 \mathrm{~b}$ at the KBP concave face, as well as $\alpha \mathrm{HP} 5 \mathrm{a}$, all strongly reduced KBP's inhibition of both KIF15_MDC and KIFIA_MDC peroxisome translocation activity (Fig 5D-G). This can be seen by the extent of peroxisome translocation and in an increase of peroxisomes in the cell periphery after rapalog addition, similar to the control condition without KBP (Fig 5D-G). Also, as observed in the pull-down assay, mutation of L1, L3, or L5 had no effect on KBP's inhibition of KIF15 MDC or KIF1A MDC (Fig 5D-G). This reinforces the conclusion that while these elements are close enough to form contacts with the parts of the kinesin motor domain in our reconstruction, they do no contribute significantly to KBP inhibition of kinesin-mediated translocation in cells.

A
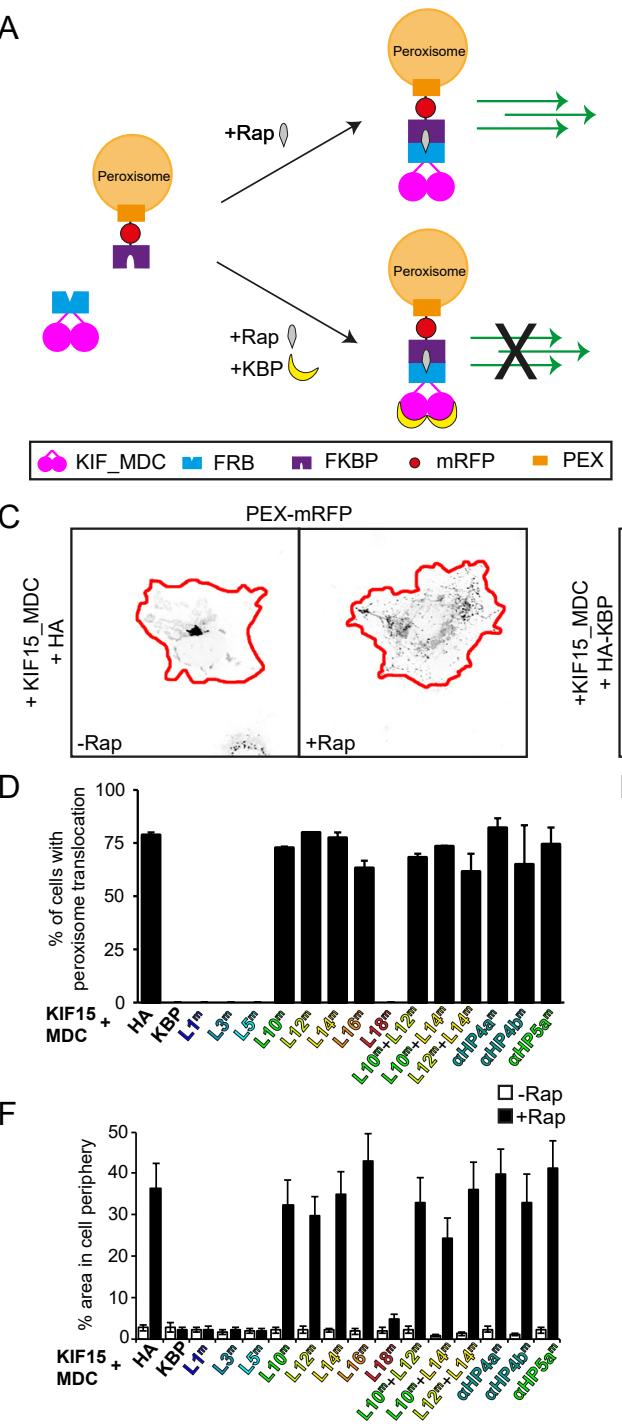
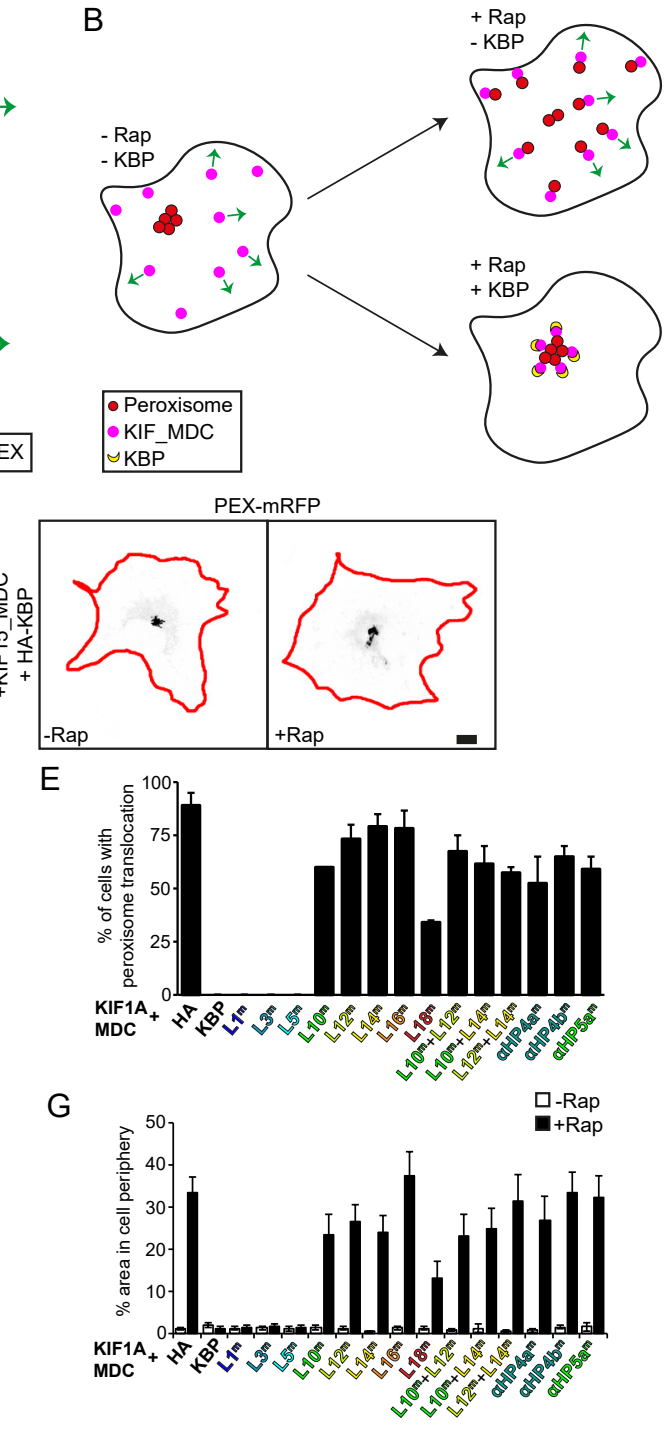

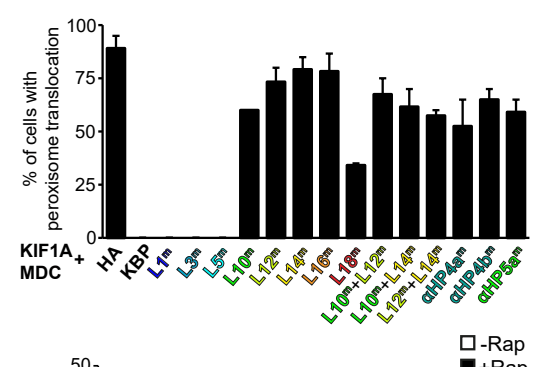

Figure 5. Disruption of cryo-electron microscopy defined kinesin-binding protein (KBP)-kinesin interface perturbs KBP inhibition of KIFL- and KIFIA-mediated cargo translocation in cells

(A) Schematic depiction of the inducible peroxisome motility assay, with the kinesin motor domain fused to an FRB domain and PEX fused to an FKBP domain. Addition of rapalog (Rap) links FRB and FKBP and induces peroxisome translocation by kinesin dimers. Expression of KBP inhibits kinesin movement, such that addition of rapalog canno Without rapor or KBP, peroxisomes localise in the cell centre, whereas kinesin moves towards the cell periphery. Rapalog induces peroxisome translocation into the cell periphery, which is inhibited in the presence of KBP. (C) Representative images of peroxisomes in COS-7 cells expressing KIF15 MDC-FRB, PEX-mRFP-FKBP, and HA (left panels) or HA-KBP (right panels) without and with addition of rapalog. Scale bar: $10 \mu \mathrm{m}$. (D,E) Quantification of the percentage of cells in which peroxisome translocation is observed after rapalog treatment in cells expressing KIF15_MDC-FRB (D) or KIF1A_MDC-FRB (E), PEX-mRFP-FKBP, and HA-KBP constructs including the indicated mutants. Data are displayed as mean \pm s.e.m. (n=28-35 cells from two independent experiments). $(\mathbf{F}, \mathbf{G})$ Quantification of the area above threshold intensity in the outer $5 \mu \mathrm{m}$ (KIFIA_MDC) or $7.5 \mu \mathrm{m}$ (KIF15_MDC) o the cell from the total area above threshold intensity in cells expressing KIFT__MDC-FRB (F) or KIF1A_MDCFRB (G), PEX-mRFP-FKBP, and HA-KBP constructs including the indicated mutants without and with rapalog 
Mutation of either of L12 or L14 strongly abrogated KBP inhibition of KIF15 MDC/KIF1A MDC-based translocation, a more pronounced effect than was seen in the pulldown assay. Similarly, although mutation of L10 and L16 had no effect on KBP-kinesin interaction in the pull-down assay, mutation of these loops disrupted KBP inhibition of KIF1A MDC and KIF15 MDC in the translocation assay (Fig 5D-G). A subset of the above described mutations was also combined to assess additive effects. Here we observed that $\mathrm{KBP}$ constructs containing mutations in both $\mathrm{L} 10+\mathrm{L} 12, \mathrm{~L} 10+\mathrm{L} 14$, or $\mathrm{L} 12+\mathrm{L} 14$ had similar effects to KBP with only one of the loops mutated (Fig 5D-G), suggesting that KBP inhibition is more readily disrupted in the translocation assay.

Interestingly, while all the above described regions affected KBP inhibition of KIF15 MDC and KIF1A MDC equivalently, the L18 KBP mutant inhibited KIF15 MDC equivalent to wild type, but only exhibited partial inhibition of KIF1A_MDC-mediated translocation. It should, however, be noted that fewer cells show peroxisome translocation by KIF1A MDC when L18 is co-expressed (Fig 5E), suggesting that the L 18 KBP mutant also The L18 mutant is the single example of contradictory behaviours between the assays, because it appeared to enhance KBP-kinesin binding in the pull-down assay. Our structural data do not provide a clear rationale for this, and future studies will investigate the role of this region of KBP further and, for example, whether it is subject to post-translational regulation that could regulate KBP's inhibitory activity.

However, taken together, the translocation and pull-down assays both demonstrate the functional importance of the kinesin interaction with the TPR-containing $\alpha$-helices at the KBP concave face and the set of loops in the KBP C-terminal subdomain. The translocation and pull-down assays also reveal differences in the sensitivity of the KBP-kinesin interaction to perturbation, where translocation is more readily disrupted than the interactions detected by pull-down. These differences likely reflect the greater complexity of motor regulation during active translocation and could be a function of FRB-FKBP-mediated motor dimerisation. It might also reflect the fact that cellular MTs in the translocation assay can directly compete might also reflect the fact that cellular MTs in the translocation assay can directly compete
with KBP for kinesin binding. Overall, these mutation studies support the idea that KBP interacts with different kinesin family members in a similar way via an extended interface at KBP's concave face that is composed of TPR-containing $\alpha$-helices and $\alpha$-solenoid edge loops, particularly in the C-terminal subdomain.

\section{Specific sequences in the tubulin-binding subdomain are conserved across}

\section{KBP-binding kinesin family members}

Given that KBP selectively binds and inhibits only a subset of kinesins (Kevenaar et al., 2016), we used our structural data to investigate the basis of this selectivity. Although the resolution of our reconstruction and the flexibility of some loops do not provide a detailed molecular description of the interaction interface, our structure shows that the kinesin tubulin-binding subdomain is the key KBP-interacting region. Analysis of the sequences of this region in KBP-binding and KBP-non-binding kinesins (Fig 6A) revealed patterns of this region in KBP-binding and KBP-non-binding kinesins (Fig 6A) revealed patterns of sequence conservation across the entire subdomain in all KBP-binding kinesins; this included
both the K 35 -KL8 and KL11-K $\alpha 4-\mathrm{KL} 12-\mathrm{K} \alpha 5-\mathrm{KL} 13$ regions. In contrast, the equivalent both the K $\beta 5-K L 8$ and KL11-Ka4-KL12-Ka5-KL13 regions. In contrast, the equivalent
regions are more variable in kinesins that are not inhibited by KBP. The length of KL8, which oins the two K $\beta 5$ strands, was also consistently five residues long in KBP-binding kinesins, while it was variable in KBP-non-binding kinesins. From our KBP-KIF15_MD6S structure, the sensitivity of the KBP interaction to KL8 length makes sense considering the tight fit of this loop between KBP L12 and L14 (Fig 6B). In summary, two consensus motifs in KL11Ka4-KL12-K $\alpha 5-K L 13$ and K $\beta 5-K L 8$ regions of the tubulin-binding subdomain are found in KBP-binding kinesins and these are likely to form the basis of KBP's kinesin family member selectivity. We therefore propose a model where KBP selects and inhibits target kinesins through binding and remodelling a compatible tubulin-binding subdomain, obstructing the kinesin MT-binding surface (Fig 6C).

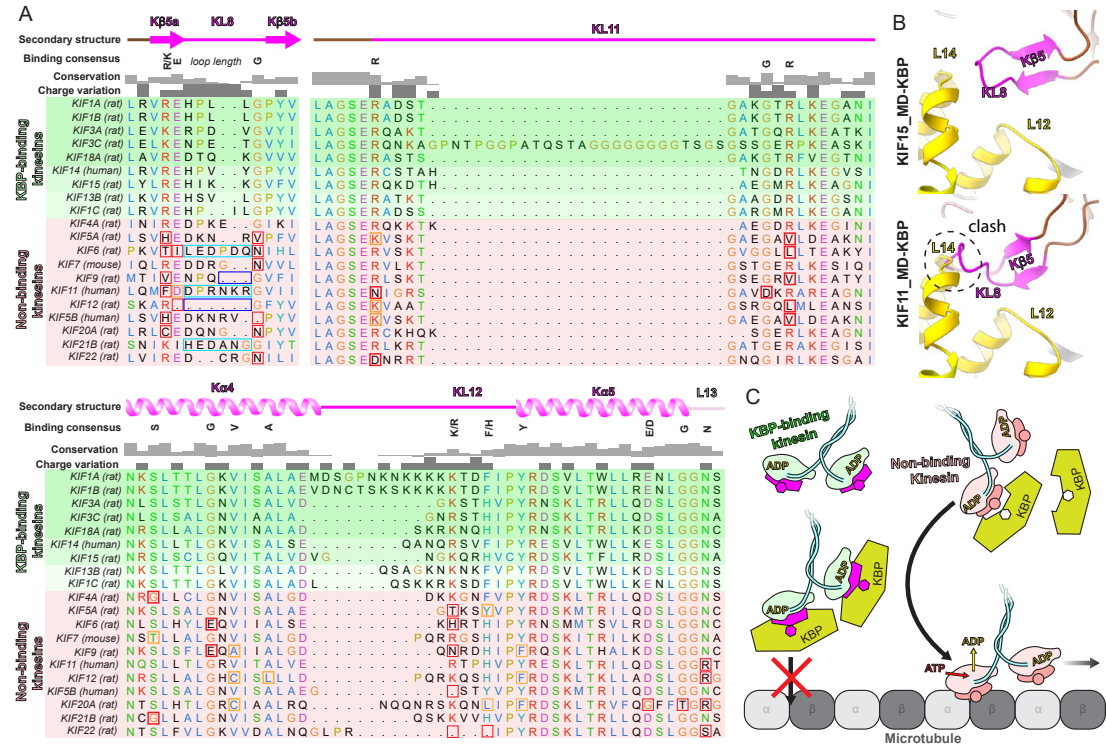

igure 6. Conserved motifs in kinesin-binding protein (KBP)-binding kinesin MDs

(A) Sequence alignment of the tubulin-binding subdomain from kinesin motor domains, made using Clustal Omega multiple sequence alignment (Sievers et al., 2011). Residues are coloured according to standard Clustal X colouring (dependent on residue type and conservation, see http://bioinfolab.unl.edu/emlab/documents/clustalx_doc/clustal. html\#C). Kinesin MD constructs experimentally assessed for KBP interactivity are taken from Kevenaar et al. 2016; strongest interactors are in rows highlighted in darker shades of green, weaker interactors in lighter shades well as a 'binding consensus' column indicating residues/loop length conserved at the interface (according to the KBP-KIF15 MD6S complex) in KBP-binding but not non-binding kinesins are shown above the alignment Nonconservation relative to this consensus is shown in boxed sequence; red boxes, non-conservative substitutions orange boxes, conservative substitutions (general charge/polarity/hydrophobicity retention), cyan boxes, extended loop region, dark blue boxes, truncated loop region. (B) Top panel; view of the K $\beta 4-K L 8$ region of the tubulinbinding subdomain in the KBP-KIF15_MD6S model, coloured as in Fig 4. Bottom panel; as in upper panel, but with the KIF11_MD cryo-electron microscopy model (PDB: 6TA4 [Peña et al., 2020]) superimposed onto the now hidde KBP-KIF15_MD6S. Note steric clash introduced by KIF11_MD's extended KL8. (C) Schematic model of KBP's hypothesised selective kinesin inhibition mechanism. KBP (olive) binds the compatible TBsd of recognised kinesin sterically blocks the TBsd interaction with MTs (grey), preventing activation of kinesin ATPase and motility

\section{DISCUSSION}

In this study, we reveal the TPR-containing right-handed $\alpha$-solenoid structure of the $\sim 72 \mathrm{kDa}$ KBP using VPP cryo-EM. At the time of writing and to our knowledge, structures of only a few macromolecular complexes $<80 \mathrm{kDa}$ have been determined using cryo-EM (Merk et al., 2016; Zhang et al., 2018; Khoshouei et al., 2017; Herzik et al., 2019; Fan et al., 2019). The structure of the KBP-KIF15 MD6S complex shows how KBP binds the KIF15 MD6S via its concave face and undergoes subtle remodelling of its N-terminal domain to accommodate kinesin binding. This further reinforces the idea that the TPR-containing structures are not simply static scaffolds but can flexibly respond to ligand binding (Pernigo et al., 2018). In contrast, the KIF15 motor domain undergoes a radical conformational change in forming a complex with KBP, in which helix $\alpha 4$, the major component of the motor's tubulin-binding subdomain, is displaced from the main MD body by $\sim 15 \AA$ into the KBP concave face. This is consistent with previous observations that this region of the kinesin motor domain being rather malleable and able to move independently of the core structure of kinesin motor domains (Wang et al., 2016a; Scarabelli and Grant, 2013). Our evidence suggests this observation is not due to use of a cysteine-substituted KIF15 motor domain construct because: 
(i) this protein exhibited equivalent MT-stimulated ATPase activity compared to nonsubstituted KIF15 MD (Fig 2-S1A), (ii) it exhibited structurally canonical MT binding and response to nucleotide (Fig 3-S1), and (iii) the substituted residues are not well conserved among KBP-binding kinesins (Fig 6A). The large displacement of helix $\alpha 4$ expands the surface area over which the normally compact tubulin-binding subdomain of the kinesin motor domain can interact with KBP, and it is through the sequence and shape of this interface that the selectivity of KBP for a subset of kinesin motors is presumably defined.

Analysis of the KBP-KIF1A_MD complex (Fig 2-S1C) supports the idea of a conserved mode of interaction between a subset of kinesins and the concave face of KBP. Interestingly, KIF1A_MD exhibited flexibility in its interaction with KBP, which was no observed in the KBP-KIF15_MD6S complex. Whether this reflects a physiological reality, or a result of the EM preparation method is uncertain at present, although we think it unlikely to be due to our use of KIF15 MD6S. However, our 2D classifications combined with mutation studies strongly suggest KIF15 MD6S and KIF1A MD share an overall similar KBPbinding mode.

Targeted mutations to the various kinesin-binding KBP elements reduced complex affinity, yet no single mutation completely disrupted the interaction in our pull-down assays (Fig 5-S2). In contrast, in the context of active MT-based cargo translocation in the cellular environment, KBP inhibitory activity appeared more sensitive to disruption (Fig 5D-G). Although most KBP mutations that have been reported in GOSHS result in total loss of protein (Brooks et al., 2005; Dafsari et al., 2015; Valence et al., 2013; Salehpour et al., 2017), a recent study details missense mutations that only partially reduce protein expression (MacKenzie et al., 2020). Our data illustrate that KBP's activity is additionally sensitive to mutations in key regions that affect its ability to bind kinesins.

Our structure shows that KBP binds exclusively to the tubulin-binding subdomain of KIF15 MD6S, sterically preventing MT attachment. The interaction of kinesin motor domains with the MT surface via its tubulin-binding subdomain stimulates nucleotide exchange and kinesin ATPase activity. In contrast, on interaction with KBP, the displacement of helix $\alpha 4$ from the kinesin nucleotide-binding site, together with the absence of MT-mediated ordering of KL9 and KL11, means that its catalytic site is distorted and the structural changes associated with MT-stimulated ATPase cannot occur. This could be an important facet of the role of KBP in the energy economy of the cell in addition to directly blocking kinesin-MT interactions.

The concave face of TPR-containing $\alpha$-solenoids commonly serve as a recognition platform for specific peptide motifs, including those forming $\alpha$-helical structures (PerezRiba and Itzhaki, 2019). Specificity and affinity for target motifs are determined in part by the shape of the $\alpha$-solenoid concave face, which in turn is defined by the fold's supertwist. (Zeytuni surface or $\alpha$-solenoid edge (Zeytuni and Zarivach, 2012). KBP kinesin specificity and affinity are defined by the interaction of its concave face with the large surface area of the kinesi L11-K $\alpha 4-K L 12-K \alpha 5-K L 13$ region, in addition to binding the kinesin K $\beta 5-K L 8$ region a its $\alpha$-solenoid edge. Interestingly, distal to the N-terminal MT-binding region of kinesin- 1 C-terminally associated kinesin light chain use their unique TPR-containing $\alpha$-solenoid concave face to select cargos via recognition of specific peptide motifs (Cross and Dodding, 2019). Therefore, peptide selectivity by TPR-containing $\alpha$-solenoids is a facet of both kinesin MT-binding and cargo-binding regulatory mechanisms. Such protein-protein interaction may be selectively targeted for disruption (Randall et al., 2017), and the insights arising from our work provide future avenues to disrupt KBP-kinesin interactions and thereby explore $\mathrm{KBP}$ interactions and regulatory roles.

The effective and selective kinesin inhibitory mechanism of KBP revealed by our work may fulfil specific roles in the kinesin regulatory toolbox employed by cells to spatially and temporarily orchestrate kinesin activity. Future studies will be aimed a understanding how KBP interacts with kinesins in dimeric and/or autoinhibited forms.
For example, while KBP does not interact with some target kinesins in autoinhibited conformations (Malaby et al., 2019), others that retain a structurally available tubulinbinding subdomain, such as autoinhibited kinesin-3 monomers (Ren et al., 2018), are feasible binding partners. KBP can bind to constitutively active dimeric kinesin constructs that lack autoinhibitory regions (Kevenaar et al , 2016; Malaby et al , 2019), although the stoichiometry and structural details in this context are unclear. Furthermore, any additional effects of kinesin cargo binding on their susceptibility to KBP inhibition are not well understood. It will also be of key importance to elucidate the mechanisms of KBP activity regulation, for example, by phosphorylation of KBP and/or kinesins (Kevenaar et al., 2016) and KBP acetylation and targeted degradation by the ubiquitin system (Donato et al., 2017). Our structural characterisation of the KBP-kinesin inhibitory interaction provides an important mechanistic platform from which to expand our understanding of KBP's biological roles in neuronal function and cancer.

\section{MATERIALS AND METHODS}

Full length human KBP residues 1-621 in a PSTCm1 expression vector (with kanamycin resistance and a N-terminal (Nombin removed via incubation with thrombin protease overnight at $44^{\circ} \mathrm{C}$. The protein was then subjected to reverse IMAC and further purified using size exclusion chromatography (SEC) into a buffer of $20 \mathrm{mM}$ Tris- $\mathrm{HCl}(\mathrm{pH} 7.4), 150 \mathrm{mM}$ $\mathrm{NaCl}, 2.5 \mathrm{mM} \mathrm{CaCl}$, and $1 \mathrm{mM}$ DTT. Protein was snap-frozen and stored in at $-80^{\circ} \mathrm{C}$.

A human KIF15 motor domain and neck linker construct (residues 1-375) in a pET21a vector with C-terminal $6 \times$ His-tag was generated by chemical synthesis (GenScript, Piscataway, NJ). Six of the eight cysteine residues (C5S, C50S, C162S, C294S, C314S, and C346S) were mutated and two cysteines were inserted (S250C and G375C) KIF15_MD6S was expressed and purified using methods previously described (Rosenfeld et al., 2005), then buffer exchanged into $25 \mathrm{mM}$ HEPES pH 7.5, $100 \mathrm{mM} \mathrm{KCl}, 2 \mathrm{mM} \mathrm{MgCl} 2,1 \mathrm{mM}$ EGTA, $1 \mathrm{mM}$ DTT, $0.1 \mathrm{mM}$ ATP, sna frozen in liquid nitrogen, and stored at $-80^{\circ} \mathrm{C}$.

A human KIF1A motor domain and neck linker construct (KIF1A_MD residues 1-362) in a pFN18a Gold (DE3) cells, as previously described (Athert Halo-tag and a C-terminal $6 \times$ His-tag) was expressed in BL21Halo-tag was removed via incubation overnight with TEV protease at $4^{\circ} \mathrm{C}$. The protein was then isolated from TEV via a second IMAC with Ni-NTA resin and further purified by SEC into a storage buffer of (20 mM HEPES, pH 7 , $150 \mathrm{mM} \mathrm{NaCl}, 5 \mathrm{mM} \mathrm{MgCl} l_{2}, 0.1 \mathrm{mM} \mathrm{ADP}$, and $1 \mathrm{mM} \mathrm{TCEP}$.

KIF15 MD6S or KIF1A MD complexes with KBP were purified via IMAC using the $6 \times$ His-tag on the kinesin constructs. Briefly, His-tagged kinesins were incubated with a 10 times excess of KBP in $20 \mathrm{mM}$ Tris-HC (pH 7.5), $150 \mathrm{mM} \mathrm{NaCl}, 1 \mathrm{mM} \mathrm{MgCl}{ }_{2}, 10 \mathrm{mM}$ midazole, $1 \mathrm{mM}$ DTT, and $0.2 \mathrm{mM}$ ADP for $5 \mathrm{~min}$ at $4^{\circ} \mathrm{C}$. Following IMAC, complexes were eltud from the Ni-NTA resin (Qiagen) by addition of $200 \mathrm{mMM}$ imidazole, then dialysed $4^{\circ} \mathrm{C}$ for $4 \mathrm{hr}$ into $20 \mathrm{mM}$ Tris- $\mathrm{HCl}(\mathrm{pH} 7.5), 150 \mathrm{mM} \mathrm{NaCl}, 1 \mathrm{mM} \mathrm{MgCl}_{2}, 1 \mathrm{mM}$ DTT, and $0.2 \mathrm{mM} \mathrm{AD}$.

Steady-state ATPase assay

ATPase activity of KIF15_MD6S was measured in ATPase buffer (50 mM potassium acetate, $25 \mathrm{mM} \mathrm{HEPES,} 5 \mathrm{mM}$ magnesium acetate, and $1 \mathrm{mM}$ EGTA, $\mathrm{pH} 7.50$ ) by measuring phosphate production in the presence of a minimum of a fivefold molar excess of paclitaxel-stabilised MTs, using a commercially available kit (EnzChek, Molecula

ample preparation for cryo-EM

KBP was prepared for cryo-EM using three different approaches. In the first approach, KBP was diluted to 0.15 glow-discharged C-flat $2 / 2$ holey carbon EM grids (Protochips, Morrisville, NC). For the and $4 \mu \mathrm{l}$ were applied was diluted to $0.3 \mathrm{mg} / \mathrm{ml}$ in $\mathrm{KBP}$ dilution buffer and $4 \mu \mathrm{l}$ were applied to glow-discharged 1.2/1.3 AuFoil gold gride (Quantifoil). For the third approach, glow-discharged C-flat 2/2 holey carbon EM grids were coated with graphencoxide (GO) according to the protocol described by Cheng and colleagues (Cheng et al.,2020) and then 4 $\mathrm{ul}$ of KBP diluted to $0.02 \mathrm{mg} / \mathrm{ml}$ in KBP dilution buffer were added.

Kinesin motor domain-KBP complexes were diluted to $0.03 \mathrm{mg} / \mathrm{ml}$ in KBP-kinesin dilution buffer $(20$ $\mathrm{mM}$ Tris-HCl [pH7.5], $50 \mathrm{mM} \mathrm{NaCl}, 1 \mathrm{mM} \mathrm{MgCl}, 1 \mathrm{mM}$ DTT, and $0.2 \mathrm{mM} \mathrm{ADP}$ ) and $4 \mu \mathrm{l}$ were added to the GOcoated gold grids described above. After a $30 \mathrm{~s}$ incubation of samples on the EM grid in a Vitrobot Mark IV (F, samples were blotted (6-8 s, blot force- 10$)$ and vitinited in liquid ethane. All steps were performed at $4^{\circ} \mathrm{C}$ 
For preparation of the KIF15_MD6S-MT complex, porcine tubulin ( $>99 \%$ pure, Cytoskeleton Inc) was polymerised in MES polymerisation buffer ( $100 \mathrm{mM} \mathrm{MES,} 1 \mathrm{mM} \mathrm{MgCl}_{2}, 1 \mathrm{mM} \mathrm{EGTA}$, and $1 \mathrm{mM}$ DTT, $\mathrm{pH}$ 6.5) with $\mathrm{mM} \mathrm{GTP}$ at $37^{\circ} \mathrm{C}$ and then stabilised with $1 \mathrm{mM}$ paclitaxel. Approximately $70 \mu \mathrm{M} \mathrm{KIF15}$ MD $6 \mathrm{~S}$ was pre-incubated fo $5 \mathrm{~min}$ with $5 \mathrm{mM}$ of AMPPNP in BRB 80 at room temperature, and then mixed with $20 \mu \mathrm{M}$ stabilised MTs. After a furthe incubation of $15 \mathrm{~min}, \mathrm{a} \mu \mathrm{\mu l}$ droplet was applied to a pre-glow discharged holey carbon grid ( $2 / 2 \mathrm{C}$-flat, Protochips Inc),
blotted for $3.5 \mathrm{~s}$, and then vitrified in liquid ethane using a Vitrobot Mark IV at ambient temperature and $80 \%$ humidity. Cryo-EM data collection

Tre (Therm zero-loss imaging mode.

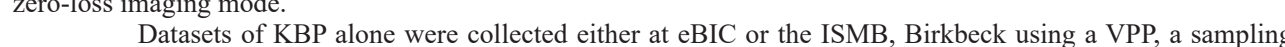
of $\sim 1.05 \AA /$ pixel and a nominal defocus range of $0.5-0.7 \mu \mathrm{m}$. The total dose was $42 \mathrm{e}-/ \AA 2$ over 40 frames, with the detector operating in counting mode at a rate of $\sim 5 \mathrm{e}-/ \mathrm{pixel} / \mathrm{s}$.

Datasets of KBP-kinesin complexes were collected at the ISMB, Birkbeck without a phase plate and a nominal defocus range of 1.5-4 $\mu \mathrm{m}$. KIF1A_MD-KBP complexes were collected at a sampling of $0.85 \AA / \mathrm{pixe}$ whereas KBP-KIF15_MD6S complexes were collected at a sampling of $1.047 \AA /$ pixel. For KIF1A_MD-KB complexes, the total dose was $88 \mathrm{e}-/ \AA 2$ over 36 frames, with the detector operating in counting mode at a rate of $7.1 \mathrm{e}-/ \mathrm{pixel} / \mathrm{s}$. For KBP-KIF15_MD6S complexes, the total dose was $80 \mathrm{e}-/ \AA 2$ over 64 frames, with the detector operating in counting mode at a rate of $5.7 \mathrm{e}-/ \mathrm{pixel} / \mathrm{s}$

The KIF15_MD6S-MT dataset was collected manually on a Tecnai Polara microscope (Thermo Fisher) at the ISMB, Birkbeck, operating at $300 \mathrm{kV}$, with a K2 summit direct electron detector (Gatan, CA, USA) and a quantum post-column energy filter (Gatan) operated in zero-loss imaging mode. A nominal defocus range of 1.0-3. in counting mode at a rate of $6.2 \mathrm{e}-/ \mathrm{pixel} / \mathrm{s}$.

\section{Cryo-EM data processing}

Low-dose movies were motion-corrected using MotionCor2 (Zheng et al., 2017) with a patch size of 5, generating and then dose-weighted sums were used for all further was performed on full-dose sums with gCTF (Zhang, 2016) all micrographs with $\mathrm{gCTF}$ resolutions worse than $4.5 \AA$, as estimated with a custom cross-correlation coefficien cutoff (Python script kindly shared by Radostin Danev), then manually removing micrographs with poor appearance (ice contamination, protuin aggregat Ro, or poor GO coverage) in real or reciprocal space. For KBP alone data, micrographs with calculated phase shifts outside the expected phase shift progression at each plate position wer also excluded.

Particles were first picked using Eman2's neural network picker (Bell et al., 2018), with a 180 pixel box

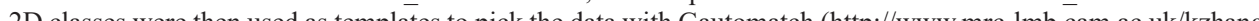
For Eman rounds of 2D classification were performed in RELION v3.0 (Zivanov et al., 2018), cryoSPARC2 (Punjani et al, 2017), or cisTEM (Grant et al., 2018). This resulted in a total of six sets of good 2D classes showing clear secondary structure for each dataset, two produced by each programme for each picking method. For each dataset, these six goo $2 \mathrm{D}$ class sets for each dataset were then combined and duplicate particles removed. At this stage, for each sample (KBPalone, KBP-KIF__MD6S, or KBP-KIF1A_MD) good 2D classes from their constituent datasets were combined. easily combined, being from the same microscope and optical set up. However, KBP-alone data were collected on
different microscopes and had a range of pixel sizes ( $<2 \%$ difference). KBP-alone data therefore was combined at different microscopes and had a range of pixel sizes $(<2 \%$ difference). KBP-alone da
this stage using the optics grouping protocol in RELION v3.1 (Zivanov et al., 2018).

this stage using the optics grouping protocol in RELION v3.1 (Zivanov et al., 2018).
KBP-alone and KBP-KIF15 MD6S data were taken to 3D processing at this stage, while multiple attempts to process KBP-KIF1A MD data in 3D gave no reliable results. For KBP-alone and KBP-KIF15 MD6S data, de novo initial 3D models were created in cryoSPARC2. For KBP-KIF15 MD6S data, a single round of 3D classification was performed in RELION v3.0 and the best class selected and auto-refined. For KBP-alone data, 3D classification in RELION v3.1 or cryoSPARC2 did not reveal different 3D structures or improve reconstructions over sorting only in $2 \mathrm{D}$, therefore, particles selected with $2 \mathrm{D}$ classification were used as direct input for autorefinement. The final KBP-alone map was sharpened with a B-factor of -200 to the gold-standard Fourier she correlation (FSC) 0.143 cutor (4.6 A). The KBP-KIF15_MD6S map was sharpened locally with a B-factor of -495, (of local resolutions determined using RELION v3.1's inbuilt local resolution software.

The KIF15_MD6S-MT dataset was processed using our MT RELION-based pipeline (MiRP) as described previously, using low-pass filtered KIF5B_MD-decorated MTs as references (Atherton et al., 2019; Cook et al., 2020). KIFl5_MD6S 13 -protofilament-MTs were the most common MT architecture and were selected after
supervised 3D classification in MiRP for analysis. The symmetrised asymmetric unit (KIF15_MD6S plus a tubulin dimer) was locally sharpened in UCSF Chimera with a B-factor of - 134 according to local resolutions determined using RELION v3.0's inbuilt local resolution software.
All displayed 3D molecular representations were made in UCSF Chimera or ChimeraX software Pettersen et al., Cryo-EM model building and refinement

Due to low overall homology to available structures in the protein data bank (PDB), structure prediction of KBP produced poor models with little resemblance to the cryo-EM density. KBP was therefore modelled using combination of secondary structure prediction, TPR prediction, fragment homology information, prior knowledg of right-handed alpha-solenoid proteins, and with reference to the cryo-EM density

(Karpenahalli et al., 2007) available in the MPI Bioinformatics Toolkit (Zimmermann et al., 2018). Secondary structure predictions using Raptor X (Wang et al., 2016b), iTasser (Roy et al., 2010), JPred (Drozdetskiy et al., 2015), Spider2 (Yang et al., 2017), PSSpred (Yan et al., 2013), and SOPMA (Geourjon and Deléage, 1995) were then run on the sequence and consensus between these multiple predictions used to assign likely $\alpha$-helical content. To identify regions dispensable species low homology regions in KBP (from early Metazoans to humans) were determined via Clustal Omega multiple sequence alignment (Sievers et al., 2011). Finally, weak homology models for overlapping fragments of the structure were identified using the HHpred (Hildebrand et al., 2009) server in the MPI Bioinformatics Toolkit.

With the information described above a sequence alignment was built with KBP and the followin fragment homology model PDBs; 5OJ8, 4A1S, 3QC1,4NQ0, 4AIF, and 5M $\times 5$. This sequence alignment was used as a basis for multiple rounds of modelling and flexible fitting with Modeller (Sali and Blundell, 1993) and Flex-EM by consistency with the cryo-EM density and secondary structure and TPR predictions described above Finally the structure was refined against the cryo-EM density in real-space with five macro-cycles in Phenix (Afonine et al 2018). All 19 predicted and modelled helices were accounted for by rod-like cryo-EM density in the reconstruction and at $4.6 \AA$ resolution, density was discernible for bulky side chains in the TPR regions (Fig 1-S1A,B), providing a validation of the assigned sequence directionality in the fold.

The KBP-KIF15_MD6S model was built as follows: the final KBP model described above and the KIF15_MD X-ray crystallographic model (PDB code:4BN2 Klejnot et al., 2014) were rigid fitted into the KBPKIF__MD6S density in Chimera. Density for an extended a6-helix and docked neck-linker in KIF15_MD6S were absent; therefore, Modeller was used to model a short a6-helix and the neck-linker removed. A model for the L11, a4-helix, and LI2 region in KIFIS_MD6S were then created using Coot (Emsley and Cowtan, 2004) and Modeller. The model was refined into the cryo-EM density in real-space using Phenix (Afonine et al., 2018) with secondary Tough fit. Following The KIF15 MD6S-MT model was built as follows: the KIF15 MD, KIF11 MD, and KIF5B MDtubulin X-ray crystallographic models (PDB codes:4BN2 Klejnot et al, 2014; Gigant et al, 2013; Parke et al, 2010) were used as homology models in Modeller to build the KIF15 part of the complex. The KIF15 MD6S model and the paclitaxel-MT tubulin dimer model (Kellogg et al., 2017) were then rigid fitted into KIF15 MD6S-MT density, combined then refined in real-space with five macro-cycles in Phenix with peptide backbone restraints.

The following antibodies were used for immunofluorescence staining: mouse anti-HA (1:500, Roche) and goat-antiHA (1:2,000, BioLegend), rabbit anti-GFP (1.10,000, Abcam), goat anti-mouse IRDye800CW (1.15.000, LI-COR), and goat-anti-rabbit IRDye 680LT (1:20,000, LI-COR). A reagent used in this study is rapalog (AP21967, TaKaRa). mRFP-FKBP1, $\beta$-actin-Kif1A MDC-FRB (Kevenaar et al., 2016) (mouse cDNA), BirA coding vecto mRFP-FKBP1, $\beta$-actin-Kif1A_MDC-FRB (Kevenaar et al., 2016) (mouse cDNA), BirA coding vector
(van der Vaart et al., 2013), and pebioGFP (van der Vaart et al., 2013). pGW1-HA-KBP contained a linker (van der Vaart et al., 2013), and pebioGFP (van der Vaart et al., 2013). pGW1-HA-KBP contained a linker based strategies with human KBP cDNA (KIAA1279, IMAGE clone 4550085) as template and ligation into the DGW1-HA backbone. A similar strategy was used to generate the mutated KBP constructs, listed in Table 2 - 3 actinKIF15 MDC-FRB was cloned using a PCR-based Gibson Assembly strategy with mouse KIF15 cDNA as template into the $\beta$-actin-KIF1A MDC-FRB backbone. PebioGFP-KIF1A MDC and pebioGFP-KIF15 MDC were cloned into the pebioGFP backbone using PCR-based strategies with MDC-FRB constructs as templates.

\section{Cell culture, transfection, and immunofluorescence staining}

COS-7 cells were purchased from ATCC and routinely checked for mycoplasma contamination using LT07-518 Mycoalert assay (Lonza). Cells were cultured in 50/50 DMEM (Lonza)/Ham's F10 (Lonza) medium supplemented with $10 \%$ FCS (Sigma) and $1 \%$ penicillin/streptomycin (Sigma). One day before transfection cells were diluted and plated on $18 \mathrm{~mm}$ glass coverslips. COS-7 cells were transfected using FuGENE6 (Roche) following the a (PBS supplemented with 1 buffered saline (PBS) for $10 \mathrm{~min}$ at room temperature, washed three times PBS-CM (PBS supplemented with $1 \mathrm{mM} \mathrm{MgCl}$ and $0.1 \mathrm{mM} \mathrm{CaCl}_{2}$, $0.2 \%$ gelatin for $30 \mathrm{~min}$ at $37^{\circ} \mathrm{C}$, and then with primary antibodies, diluted in $0.2 \%$ gelatin, for 30 minated wit $37^{\circ} \mathrm{C}$ 
After washing three times with PBS-CM, cells were incubated for 30 min at $37^{\circ} \mathrm{C}$ with secondary antibody diluted in $0.2 \%$ gelatin, washed three times in PBS-CM, and finally mounted using Fluoromount (Invitrogen).

Cell biology image analysis and quantification

Fixed cells were imaged on a Carl Zeiss LSM 700 confocal laser scanning microscope running ZEN2011 software, using a Plan-Apochromat $40 \times 1.30$ oil DIC objective and image settings were maintained the same for all images within one experiment. Images were acquired of cells that express similar levels of HA-KBP constructs base on immunostaining (Fig 5-S1). Cells were selected on a first come first served basis. Images were processed an peroxisomes was observed, imaged cells were classified as either translocating, when peroxisomes re-localised inf the cell periphery or not translocating, when peroxisomes remained in the cell centre. For quantification of PEX translocation, an ROI of the cell area was drawn and from this a second ROI at 5 (KIF1A_MDC) or 7.5 (KIF15 MDC) $4 \mathrm{~m}$ from the outer cell area was created. Images were thresholded at 7500 (KIF15 MDC) or 10,000 (KIF1A MDC). Different peripheral areas and threshold values were defined for the two kinesins, due to observed differences in translocation properties between the kinesins (compare Fig 5-S3A-C). For the two selected ROIs, the area with fluorescent intensity above threshold was determined in the RFP channel. From these values the percentage of cell area above threshold in the cell periphery from the total area above threshold was calculated.

HEK293T cells were purchased from ATCC and routinely checked for mycoplasma contamination using LT07-518 Mycoalert assay (Lonza). Cells were cultured in 50/50 DMEM (Lonza)/Ham's F10 (Lonza) medium supplemented with $10 \%$ FCS (Signa) and $1 \%$ penicillin/streptomycin (Sigma). One day before transfection cells were diluted and plated into 6-well plates. Cells were co-transfected with pCl-Neo-BirA, HA-tagged constructs, and bioGFP-tagged constructs using MaxPEI (Polysciences) in a ratio of 3/1 PEI/DNA, according to the manufacturer's protocol. After $\mathrm{mM} \mathrm{NaCl}, 1 \%$ Triton X-100, and protease inhibitors [Roche]) for 30 min on ice. Lysates were cleared by $30 \mathrm{~m}$ centrifugation at $13.2 \mathrm{krpm}$ at $4^{\circ} \mathrm{C}$ and supernatants were incubated with blocked (incubation for 30 min at RT in $50 \mathrm{mM}$ Tris- $\mathrm{HCl} \mathrm{pH} 7.5,150 \mathrm{mM} \mathrm{KCl}$, and $0.2 \mu \mathrm{g} / \mu \mathrm{l}$ chicken egg albumin) Streptavidin Dynabeads M-280 (Invitrogen) for $1.5 \mathrm{hr}$ at $4^{\circ} \mathrm{C}$. Beads were then washed five times with washing buffer $(100 \mathrm{mM}$ Tris- $\mathrm{HCl} \mathrm{pH} 7.5$, $250 \mathrm{mM} \mathrm{NaCl}$, and $0.5 \%$ Triton X-100) and proteins were eluded from the beads by boiling for $10 \mathrm{~min}$ at $95^{\circ} \mathrm{C}$ in $2 \times$ DTT+sample buffer (20\% glycerol, 4\% SDS, $200 \mathrm{mM}$ DTT, $100 \mathrm{mM}$ Tris-HCl pH 6.8, and bromophenol blue) Protein samples were run on 10\% SDS-PAGE gels and transferred to nitrocellulose membranes (Bio-Rad) by semi-dry blotting at $16 \mathrm{~V}$ for $1 \mathrm{hr}$. Membranes were blocked by incubation in $3 \%$ bovine serum albumin (BSA) in PBS (PBS supplemented with 0.02\% Tween20) for 1 hir at room temperature. This was followed by overnight incubatio with primary antibodies in $3 \%$ BSA-PBST. Membranes were washed three times with PBST, incubated with secondary antibody in $3 \%$ BSA-PBST

\section{REFERENCES}

Afonine, P.V. et 7al. 531 . 4 . Real-space refinement in PHENIX for cryo-EM and crystallography. Acta Crystallog

Alves, M.M. et al. (2010). KBP interacts with SCG10, linking Goldberg-Shprintzen syndrome to microtubule therton, J. et a therton, J. et al. (2014). Conserved mechanism,
generation in transport kinesins. Elife 3, e 03680 ,

Atherton,J.etal.(2017).The divergentmitotickinesinMKLP2exhibitsatypical structureandmechanochemistry.Elife6 Atherton, J. et al. (2019). Structural determinants of microtubule minus end preference in CAMSAP CKK domains. Nat. Commun. 10, 5236

Bell, J.M., Chen, M., Durmaz, T., Fluty, A.C. \& Ludtke, S.J. (2018). New software tools in EMAN2 inspired by Biol. 204, 283-290. S.

Brooks, A.S. et al. (2005). Homozygous nonsense mutations in KIAA 1279 are associated with malformations of the

Brouwers, N., Mallol Martinez, N. \& Vernos, I. (2017). Role of Kif15 and its novel mitotic partner KBP in K-fiber

Chang, H.Y., Cheng, H.Y., Tsao, A.N., Liu, C. \& Tsai, J.W. (2019). Multiple Functions of KBP in Neural Development Underlie Brain Anomalies in Goldberg-Shprintzen Syndrome. Front. Mol. Neurosci. 12, 265.

Chen, S. et al. (2013). High-resolution noise substitution to measure overfitting and validate resolution in 3D

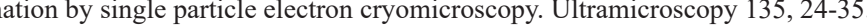

Chen, V.B. et al. (2010). MolProbity: all-atom structure validation for macromolecular crystallography. Acta

Cheng, K., Wilkinson, M., Chaban, Y. \& Wigley, D.B. (2020). A conformational switch in response to Chi converts

Cook, A.D., Manka, S.W., Wang, S., Moores, C.A. \& Atherton, J. (2020). A microtubule RELION-based pipeline for
Cross, J.A. \& Dodding, M.P. (2019). Motor-cargo adaptors at the organelle-cytoskeleton interface. Curr. Opin. Cell

Culurgioni, S., Alfieri, A., Pendolino, V., Laddomada, F. \& Mapelli, M. (2011). Inscuteable and NuMA protein bind competive 1003 .

Dafsari, H.S et al (2015). Goldberg-Shprintzen megacolon syndrome with associated sensory motor axonal neuropathy. Am. J. Med. Genet. A 167, 1300-4.

Donato, V. et al. (2017). The TDH-GCN5L1-Fbxo15-KBP axis limits mitochondrial biogenesis in mouse embryonic stem cells. Nat. Cell Biol. 19, 341-351.

Drerup, C.M., Lusk, S. \& Nechiporuk, A. (2016). KiflB Interacts with KBP to Promote Axon Elongation by

Drevillon, L. et al. (2013). KBP-cytoskeleton interactions underlie developmental anomalies in Goldberg-Shprintzen

Drozdetskiy, A., Cole, C., Procter, J. \& Barton, G.J. (2015). JPred4: a protein secondary structure prediction server.

Emsley, P. \& Cowtan, K. (2004). Coot: model-building tools for molecular graphics. Acta Crystallogr. D Biol. Fan, X. et al. (2019). Single particle cryo-EM reconstruction of $52 \mathrm{kDa}$ streptavidin at 3.2 Angstrom resolution. Nat. Commun. 10,2386

Geourjon, C. \& Deleage, G. (1995). SOPMA: significant improvements in protein secondary structure prediction by consensus prediction from multiple alignments. Comput. Appl. Biosci. 11, 681-4.

Gigant, B. et al. (2013). Structure of a kinesin-tubulin complex and implications for kinesin motility. Nat. Struct. Goddard, T.D. et al. (2018). UCSF ChimeraX: Meeting modern challenges in visualization and analysis. Protein

Grant, T., Rohou, A. \& Grigorieff, N. (2018). cisTEM, user-friendly software for single-particle image processing.

Herzik, M.A., Jr., Wu, M. \& Lander, G.C. (2019). High--1
using conventional cryo-EM. Nat. Commun. 10, 1032 .

. HHpred. Proteins 77, 128-32.

Hirokawa, N., Niwa, S. \& Tanaka, Y. (2010). Molecular motors in neurons: transport mechanisms and roles in brain function, development, and disease. Neuron 68, 610-38.

Hirokawa, N., Noda, Y., Tanaka, Y. \& Niwa, S. (2009). Kinesin superfamily motor proteins and intracellular transport. Nat. Rev. Mol. Cell Biol. 10, 682-96.

Hirst, C.S. et al. (2017). Kiflbp loss in mice leads to defects in the peripheral and central nervous system and

Karpenahalli, M.R., Lupas, A.N. \& Soding, J. (2007). TPRpred: a tool for prediction of TPR-, PPR- and SEL1-like

Kellogg, E.H. et al. (2017). Insights into the distinct mechanisms of action of taxane and non-taxane microtubule

Kevenaar, J.T. et al. (2016). Kinesin-Binding Protein Controls Microtubule Dynamics and Cargo Trafficking by Regulating Kinesin Motor Activity. Curr. Biol. 26, 849-61.

Khoshouei, M., Radjainia, M., Baumeister, W. \& Danev, R. (2017). Cryo-EM structure of haemoglobin at $3.2 \mathrm{~A}$
determined with the Volta phase plate. Nat. Commun. 8, 16099 . Klejnot, M. et al. (2014). The crystal structure and biochemical characterization of Kif15: a bifunctional molecula motor involved in bipolar spinde formation and neuronal development. Acta Crystallogr. D Biol. Crystallogr. 70

Klinman, E. \& Holzbaur, E.L.F. (2018). Walking forward with kinesin. Trends Neurosci 41, 555-556. Lawrence, C.J. et al. (2004) A standardized kinesin nomenclature. J. Cell Biol. 167, 19-22.

Lehti, M.S., Kotaja, N. \& Sironen, A. (2015). KIF1-binding protein interacts with KIF3A in haploid male germ cells.

yons, D.A., Naylor, S.G., Mercurio, S., Dominguez, C. \& Talbot, W.S. (2008). KBP is essential for axonal structure, outgrowth and maintenance
Development $135,599-608$.

Malaby, H.L.H., Dumas, M.E., Ohi, R. \& Stumpff, J. (2019). Kinesin-binding protein ensures accurate chromosome segregation by buffering KIF18A and KIF15. J. Cell Biol. 218, 1218-1234.

Mandelkow, E. \& Mandelkow, E.M. (2002). Kinesin motors and disease. Trends Cell Biol. 12, 585-9 Merk, A. et al. (2016) Breaking cryo-EM resolution barriers to facilitate drug discovery. Cell 165, 1698-1707. Miki, H., Setou, M., Kaneshiro, K. \& Hirokawa, N. (2001). All kinesin superfamily protein, KIF, genes in mouse Parke, C.L., Wojcik, E.J., Kim, S. \& Worthylake, D.K. (2010). ATP hydrolysis in Eg5 kinesin involves a catalytic 
Pena, A., Sweeney, A., Cook, A.D., Topf, M. \& Moores, C.A. (2020). Structure of microtubule-trapped human

Perez-Riba, A. \& Itzhaki, L.S. (2019). The tetratricopeptide-repeat motif is a versatile platform that enables diverse

Pernigo, S. et al. (2018). Structural basis for isoform-specific kinesin-1 recognition of Y-acidic cargo adaptors. Elife

Pettersen, E.F. et al. (2004). UCSF Chimera--a visualization system for exploratory research and analysis. J. Comput. Punjani, A., Rubinstein, J.L., Fleet, D.J. \& Brubaker, M.A. (2017). cryoSPARC: algorithms for rapid unsupervised cryo-EM structure determination. Nat. Methods 14, 290-296.

Quinaud, M. et al. (2007). Structure of the heterotrimeric complex that regulates type III secretion needle formation PNAS $104,7803-8$.

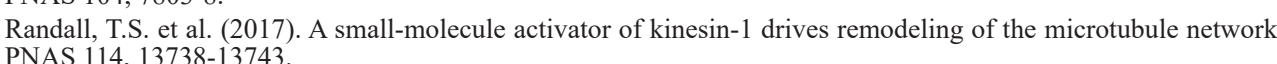

Ren, J. et al. (2018). Coiled-coil 1-mediated fastening of the neck and motor domains for kinesin-3 autoinhibition.
PNAS 115, E11933-E11942.

Rosenfeld, S.S., Xing, J., Jefferson, G.M. \& King, P.H. (2005). Docking and rolling, a model of how the mitotic
motor Eg5 works. J. Biol. Chem. 280 35684-95.

Roy, A., Kucukural, A. \& Zhang, Y. (2010). I-TASSER: a unified platform for automated protein structure and Salehpour, S., Hashemi-Gorii, F., Soltani, Z., Ghafouri-Fard, S. \& Miryounesi, M. (2017). Association of a novel nonsense mutation in KIAA1279 with Goldberg-Shprintzen syndrome. Iran J. Child Neurol. 11, 70-74.

Sali, A. \& Blundell, T.L. (1993). Comparative protein modelling by satisfaction of spatial restraints. J. Mol. Biol

Scarabelli, G. \& Grant, B.J. (2013). Mapping the structural and dynamical features of kinesin motor domains. PLoS Conin,

2012). Fiji: an open-source platform for biological-image analysis. Nat. Methods 9, 676-82. Shang, Z. et al. (2014). High-resolution structures of kinesin on microtubules provide a basis for nucleotide-gated

Sievers, F. et al. (2011). Fast, scalable generation of high-quality protein multiple sequence alignments using Clustal
Omega. Mol. Syst. Biol. 7, 539 .

Suo, C. et al. (2018). Accumulation of poten
neuroblastoma patients. Biol. Direct 13, 14 .

Tanaka, H., Ito, J., Cho, K. \& Mikawa, M. (1993). Hirschsprung disease, unusual face, mental retardation, epilepsy, and congenital heart disease: Goldberg-Shprintzen syndrome. Pediatr. Neurol. 9, 479-81.

Topf, M. et al. (2008). Protein structure fitting and refinement guided by cryo-EM density. Structure 16, 295-307. Vale, R.D. (2003). The molecular motor toolbox for intracellular transport. Cell 112, 467-80.

Valence, S. et al. (2013). Homozygous truncating mutation of the KBP gene, encoding a KIF1B-binding protein, in

van der Vaart, B. et al. (2013). CFEOM1-associated kinesin KIF21A is a cortical microtubule growth inhibitor. Dev. Cent

(2016). Motility and microtubule depolymerization mechanisms of the Kinesin-8 motor,KIF19A. Elife 5. Wang, J., Dye, B.T., Rajashankar, K.R., Kurinov, I \& \& Schulman, B.A. (2009). Insights into anaphase promoting
complex TPR subdomain assembly from a CDC26-APC6 structure. Nat. Struct. Mol Biol, 16, 987-9.

Wang, S., Li, W., Liu, S. \& Xu, J. (2016). RaptorX-Property: a web server for protein structure property prediction Wozniak, M.J., Melzer, M., Dorner, C., Haring, H.U. \& Lammers, R. (2005). The novel protein KBP regulates mitochondria localization by interaction with a kinesin-like protein. BMC Cell Biol. 6, 35.

Yan, R., Xu, D., Yang, J., Walker, S. \& Zhang, Y. (2013). A comparative assessment and analysis of 20 representative sequence alignment methods for protein structure prediction. Sci. Rep. 3, 2619.

Yang, Y. et al. (2017). SPIDER2: A Package to Predict Secondary Structure, Accessible Surface Area, and Main-

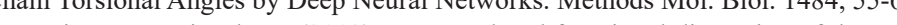

Zeytuni, N. \& Zarivach, R. (2012). Structural and functional discussion of the tetra-trico-peptide repeat, a protein Zhang. K. (2016). Gctf: Real-time CTF detern

determination and correction. J. Struct. Biol. 193, 1-12

Zhang, K. et al. (2018). Structure of the $30 \mathrm{kDa}$ HIV-1 RNA Dimerization Signal by a Hybrid Cryo-EM, NMR, and

Zheng, S.Q. et al. (2017). MotionCor2: anisotropic correction of beam-induced motion for improved cryo-electron microscopy. Nat. Methods 14, 331-332.

Zimmermann, L. et al. (2018). A Completely reimplemented MPI bioinformatics toolkit with a new HHpred derver at its core. J. Mol. Biol. 430, 2237-2243, Zivanov, J. et al. (2018). New tools for automated high-resolution cryo-EM structure determination in RELION-3.
Elife 7, e42166.

\section{SUPPLEMENTAL INFORMATION}
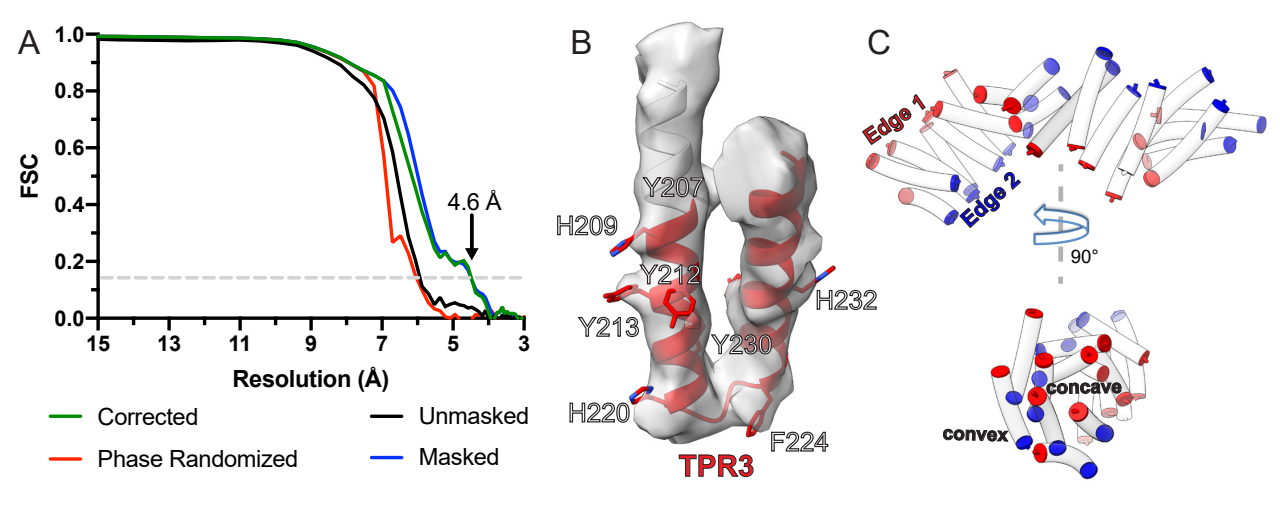

$\mathrm{D}$

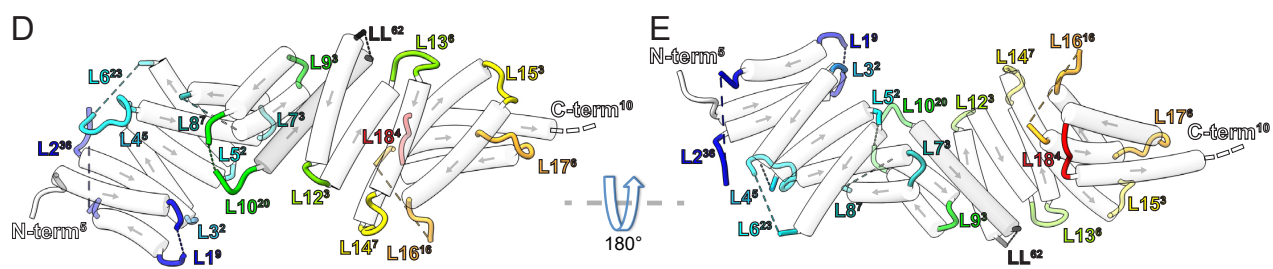

Figure 1-Supplement 1. Kinesin-binding protein (KBP) reconstruction, structure, and loop lengths

(A) Gold-standard Fourier shell correlation (FSC) curves between independent masked, unmasked, phase2018) (4.6 § resolution at the 'gold-standard' 0.143 FSC cutoff). (B) Density and fitted model for TPR3 of KBP, showing exemplar bulky side chain density that guided modelling. (C) Same view as Fig 1C (upper panel), or rotated $90^{\circ}$ around the indicated axis (lower panel), showing only $\alpha$-helices (semi-transparent white tubes) with their terminal residues coloured, illustrating the edges and faces (concave and convex) of the $\alpha$-solenoid respectively. (D) Same view as panel C, but now with loops shown and coloured (semi-transparent tube helices have their directionality represented by arrows). Each loop or terminus label has a superscript number indicating their length. (E) Same as panel D, rotated $180^{\circ}$ around the indicated axis. 


$$
\text { KBP sequence }
$$

Figure 1-Supplement 2. Kinesin-binding protein (KBP) loops, sequence, inter-species conservation, and experimental mutations

The human KBP sequence (numbering above), with residues coloured by intra-species sequence identity as indicated in the key. The following species were included in the Clustal Omega multiple sequence alignment (Sievers et al., 2011); Homo sapiens, Mus musculus Gallus gallus, Xenopus tropicalis, Alligator mississippiensis, Danio rerio, Drosophila melanogaster, Amphinodon queenslandica, Stylophora pistillata, Trichoplax adhaerens, Spizellomyces punctatus, and Salpingoeca rosetta. Above the sequence, secondary structure elements are indicated, coloured to delineate the nine $\alpha$-helical pairs and connecting loops. Mutation sites are indicated within each boxed sequence region, labelled
to coordinate with Fig 5 . Within these boxes, the mutated sequence is shown below the original wild-type sequence.

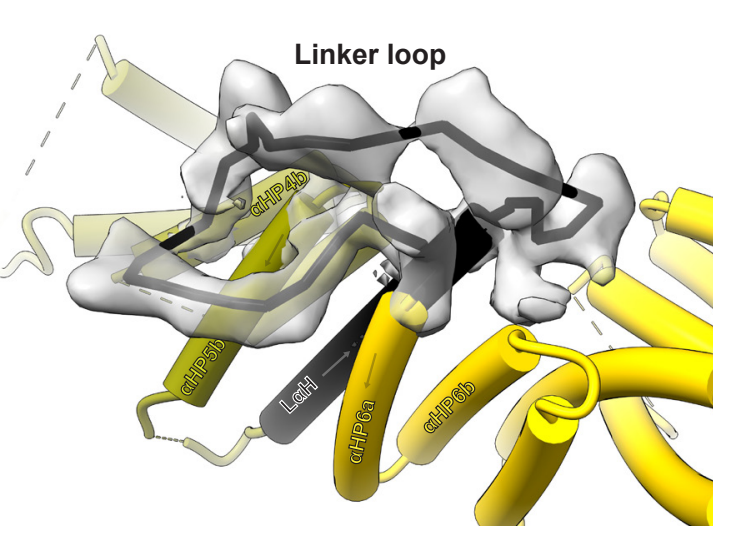

Figure 1-Supplement 3. Approximate path of the kinesin-binding protein (KBP) linker loop (LL) View of KBP"s $S L$, using the same KBP model subdomain colouring and representaton as in Fig IA,B. Only density for the $\mathrm{LL}$ is shown and a rough path for the $\mathrm{LL}$ is indicated with a solid black line. KBP $\alpha$-helices contacting the LL region are labelled.
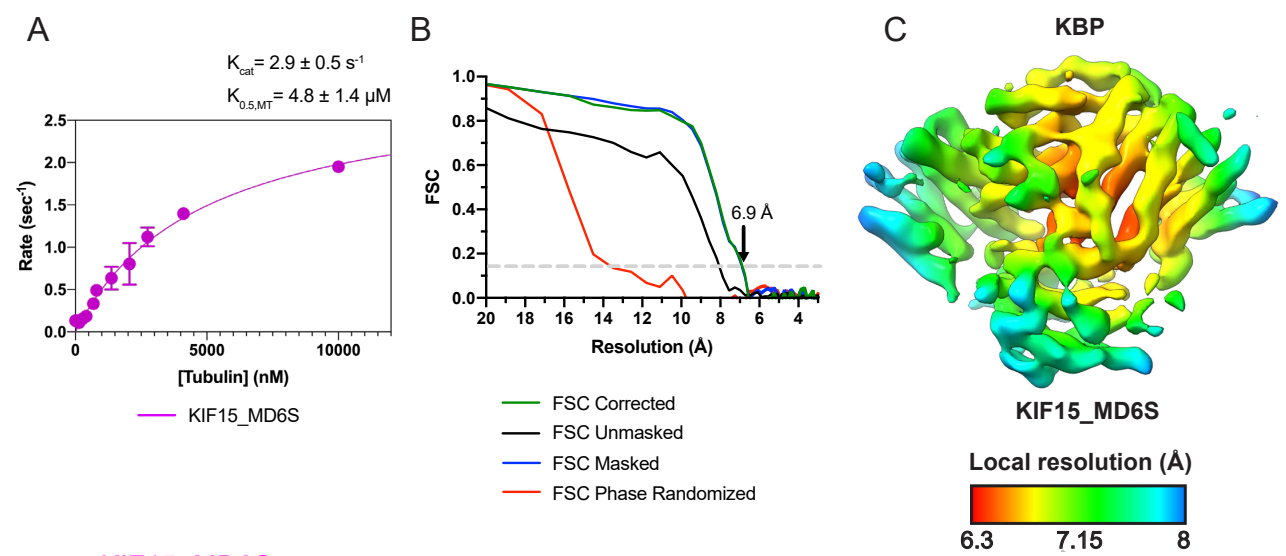

KIF15 MD6S

KIF1A MD - KBP

D

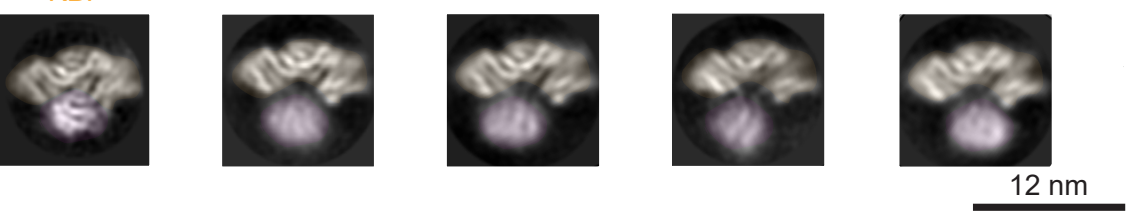

KIF15_MD6S - KBP 2D classes

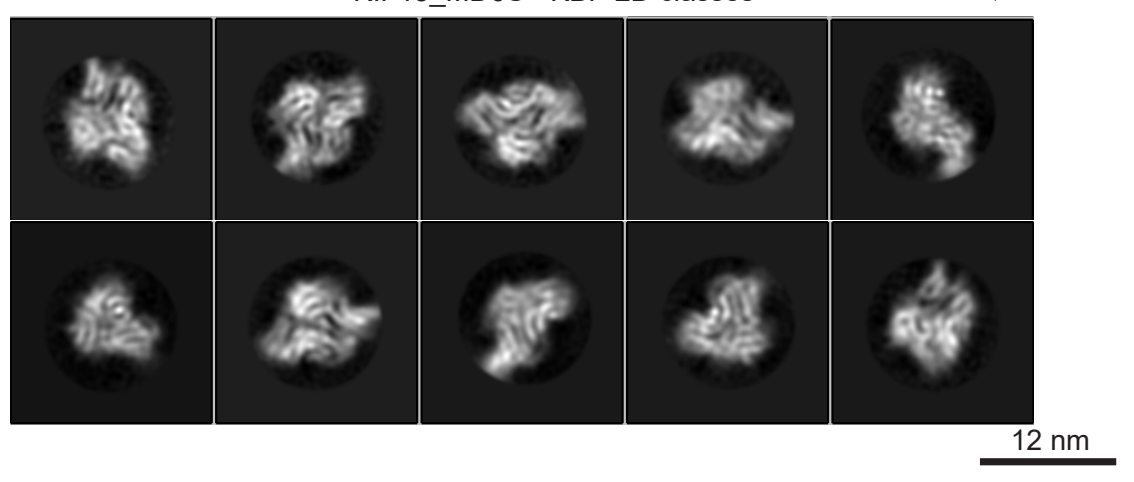

Figure 2-Supplement 1. Kinesin-binding protein (KBP)-KIF15_MD6S reconstruction resolution estimation and 2D class analysis of KBP-KIF1A_MD and KBP-KIF15_MD complexes

(A) KIF15-MD6S MT-activated steady-state ATPase velocity plotted as a function of [MT]. Data were fit to a Michaelis-Menten kinetic (pink curve) yielding values for $\mathrm{kcat}=2.9 \pm 0.5 \mathrm{~s}^{-1}$ and $\mathrm{K} 0.5, \mathrm{MT}=4.8 \pm 1.4 \mu \mathrm{M}$; R 2 M. (Klejnot et al, 2014). (B) Gold-standard Fourier shell correlation ( $\overline{\mathrm{FSC}}$ ) curves between independent maskeld unmakked, chase-romised, and corrected half-maps (Chen et al 2013) of the KBP-KIF15 MD6S complex as calculated by RELION v3.0 (Zivanov et al, 2018). The resolution at the 'gold-standard' 0.143 FSC cutoff is 6.9 $\AA$. (C) Local resolution as calculated by RELION v3.0, shown on the same view as in Fig $2 \mathrm{~A}$ with coloure density corresponding to the local resolutions indicated in the key. (D) Selected RELION v3.0 (Zivanov et al., 2018) 2D classes of KBP-KIF15 MD6S (left) and KIF1A MD-KBP (four to the right). Densities for the kinesin motor domain and $\mathrm{KBP}$ are pseudo-coloured pale magenta and pale orange respectively. Classes have been in-plane rotate KIF1A_MD. (E) A representative subset of KBP-KIF15_MD6S complex 2D classes, showing multiple orientations. 


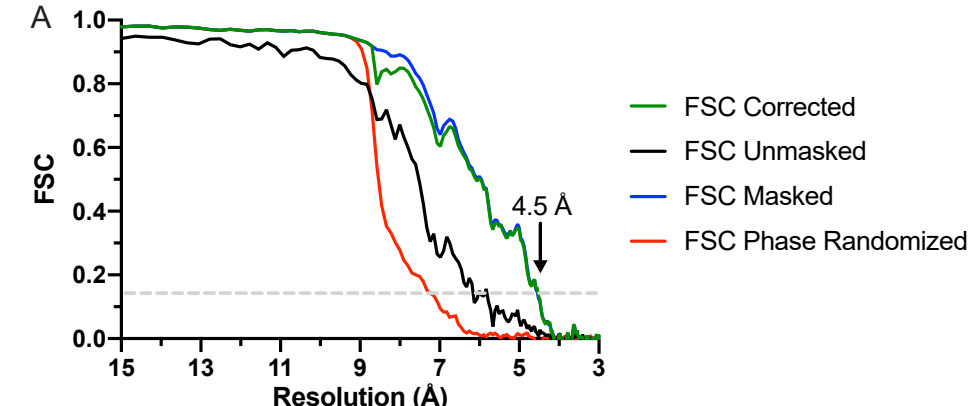

B
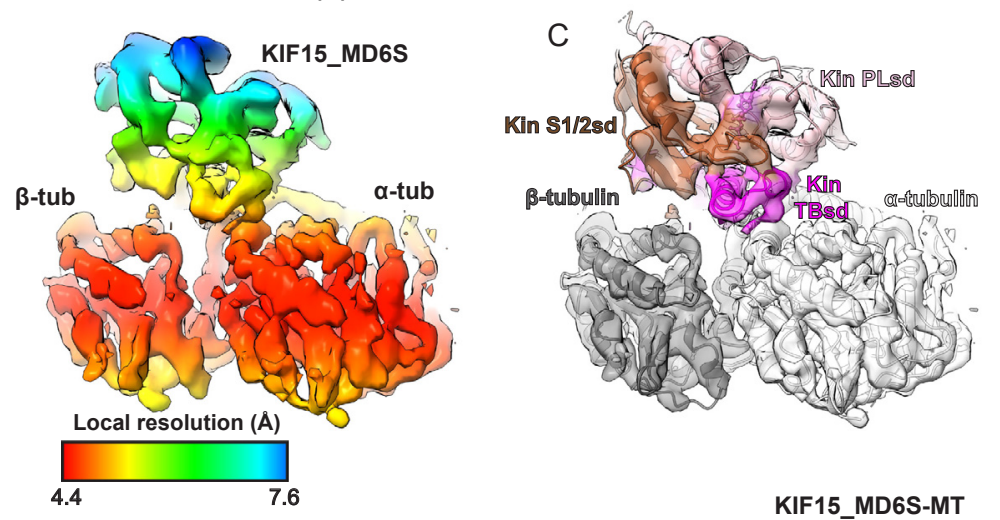

D

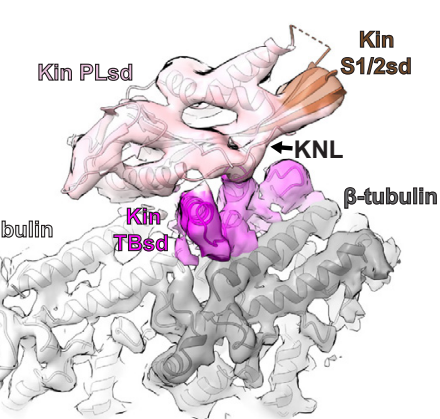

KIF15_MD
KIF15_MD6S-MT

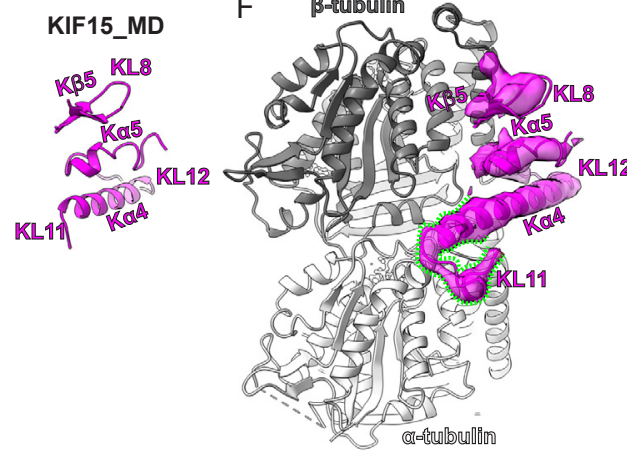

Figure 3-Supplement 1. KIF15_MD6S adopts a canonical MT-bound kinesin conformation

(A) Gold-standard Fourier shell correlation (FSC) curves between independent masked, unmasked, phaserandomised, and corrected half-maps (Chen et al., 2013) of the KIF15_MD6S-MT complex as calculated by RELION v3.0 (Zivanov et al., 2018). The resolution at the 'gold-standard' 0.143 FSC cutoff is $4.5 \AA$. (B) Loca resolution as calched by RELION v3.0, whin coloured density corresponding to the local resolutions indicated in the key. (C) The KIF_MDOS-MT asymmetric unit model in corresponding density. The KIF15_MD6S coloured by subdomain, bound $\mathrm{Mg}^{2}$-AMPPNP coloured lilac, and $\alpha$ - and $\beta$-tubulin are coloured light and dark as in ranel B. (D) A view of the KIF15 MD6S-MT asymmetric unit showing a docked conformation of the view linker (KNL). (D) A l and dest from the crystal structure (PDB code:4BN2 Klejnot et al, 2014). (F) KIF15_MDSS complexed with MTs with only the tubulin-binding subdomain and $\alpha$ - and $\beta$-tubulin shown, along with the tubulin-binding subdomain cryo-EM density (semi-transparent). Ka4 of KIF15 MD6S is extended relative to the crystal structure and KL12 adopts a new conformation on $\alpha$-tubulin, as indicated by the green dashed lines. Panels D and E show the KIF15 MD6S tubulinbinding subdomain from the same viewpoint.

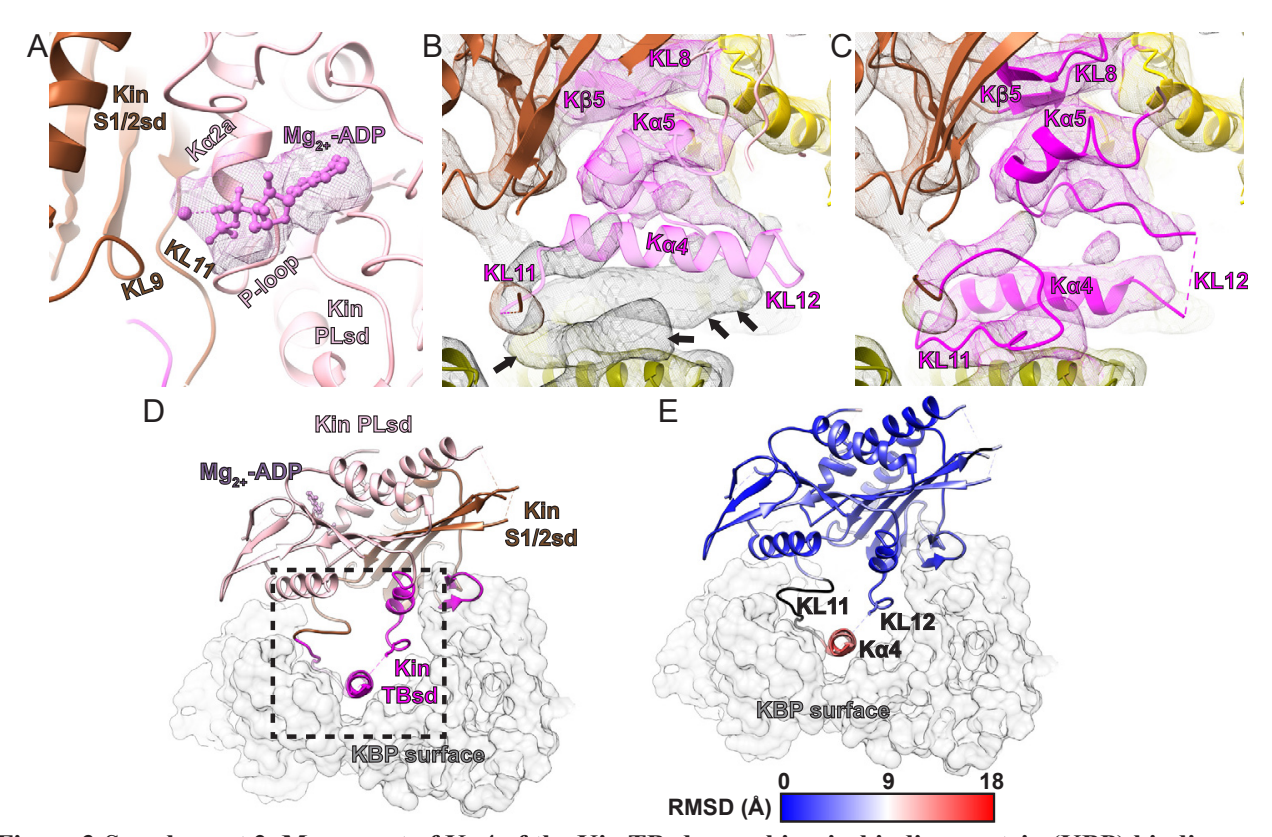

Figure 3-Supplement 2. Movement of Ka4 of the Kin TBsd upon kinesin-binding protein (KBP) binding (A) The KIF15 MD alone crystal structure (PDB code:4BN2 Klejnot et al., 2014) is shown coloured by kinesi subdomain (as in $\mathrm{Fig}$ 3), fitted into the KBP-KIF15_MD6S complex cryo-electron microscopy map, with only nucleotide-binding zoomed on the TBsd (in pale magenta to illustrate poor fit). Black (Brrows indicate unaccounted-for density. (C) As i Fig 3B, with the clipped viewpoint as in panel B of this figure (TBsd now opaque to illustrate good density fit). (D) The KBP-KIF15 MD6S model is shown as opaque ribbons, with kinesin subdomain colouring as in panels A and $\mathrm{B}$ and as labelled. KBP is shown as a transparent light grey surface representation. The boxed region indicates that shown in Fig 3C-E. (E) RMSD in $\AA$ corresponding to the KIF15_MD6S overlay in panel D, shown on the model of KIF15_MD6S in complex with KBP (grey transparent surface). Parts of the model coloured black are disordered/ missing in the KIF15_MD alone crystal structure.

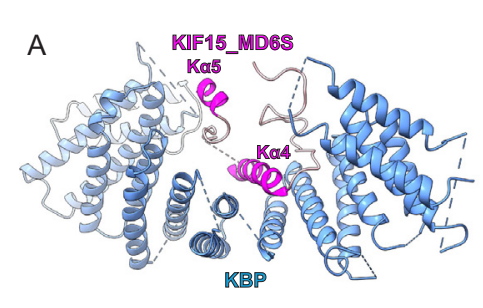

C

$$
\text { CDC26 }
$$

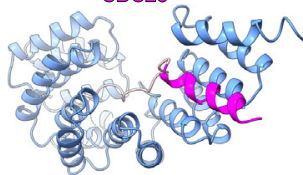

CDC10

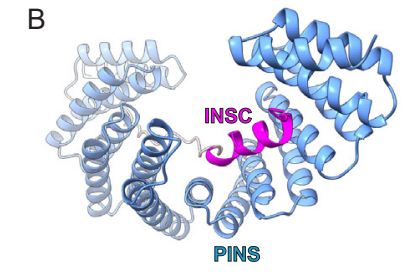

D

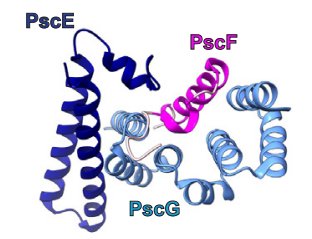

Figure 3-Supplement 3. Examples of tetratricopeptide repeat (TPR)-containing $\alpha$-solenoid proteins binding a-helical SSE ligan

(A-D) Comparison of (A) kinesin-binding protein (KBP)-KIF15_MD6S complex with other TPR-containing $\alpha$-solenoids shown in blue, and binding peptide motifs shown in magenta or pink for helical and random coil regions 2009), and (D) the PscE/PscG-PscF complex (Quinaud et al., 2007). 


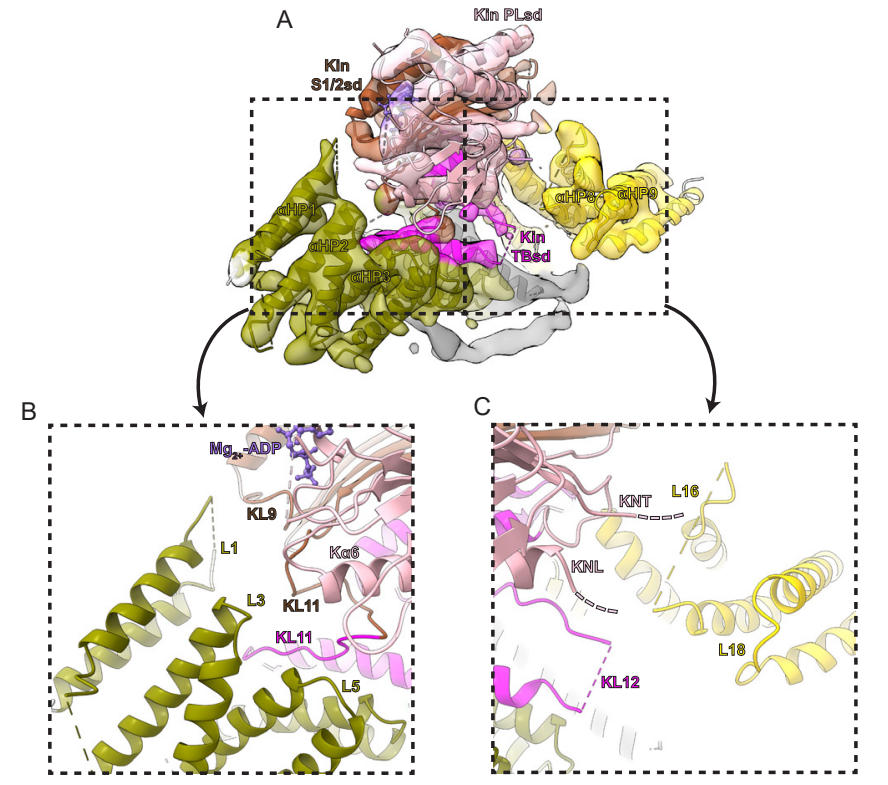

Figure 4-Supplement 1. Additional kinesin-binding protein (KBP) a-solenoid edge loops proximal to KIF15 MD6S

Colouring and representation as in Fig 4. (A) A view showing $\alpha$-helical pairs $\alpha \mathrm{HP} 1, \alpha \mathrm{HP} 2, \alpha \mathrm{HP} 3, \alpha \mathrm{HP} 8$, and $\alpha \mathrm{HP}$ of KBP and the KIF15_MD6S coloured by subdomain as labelled. (B) Left zoomed region in panel A, with density

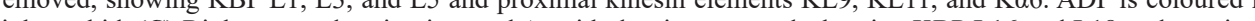

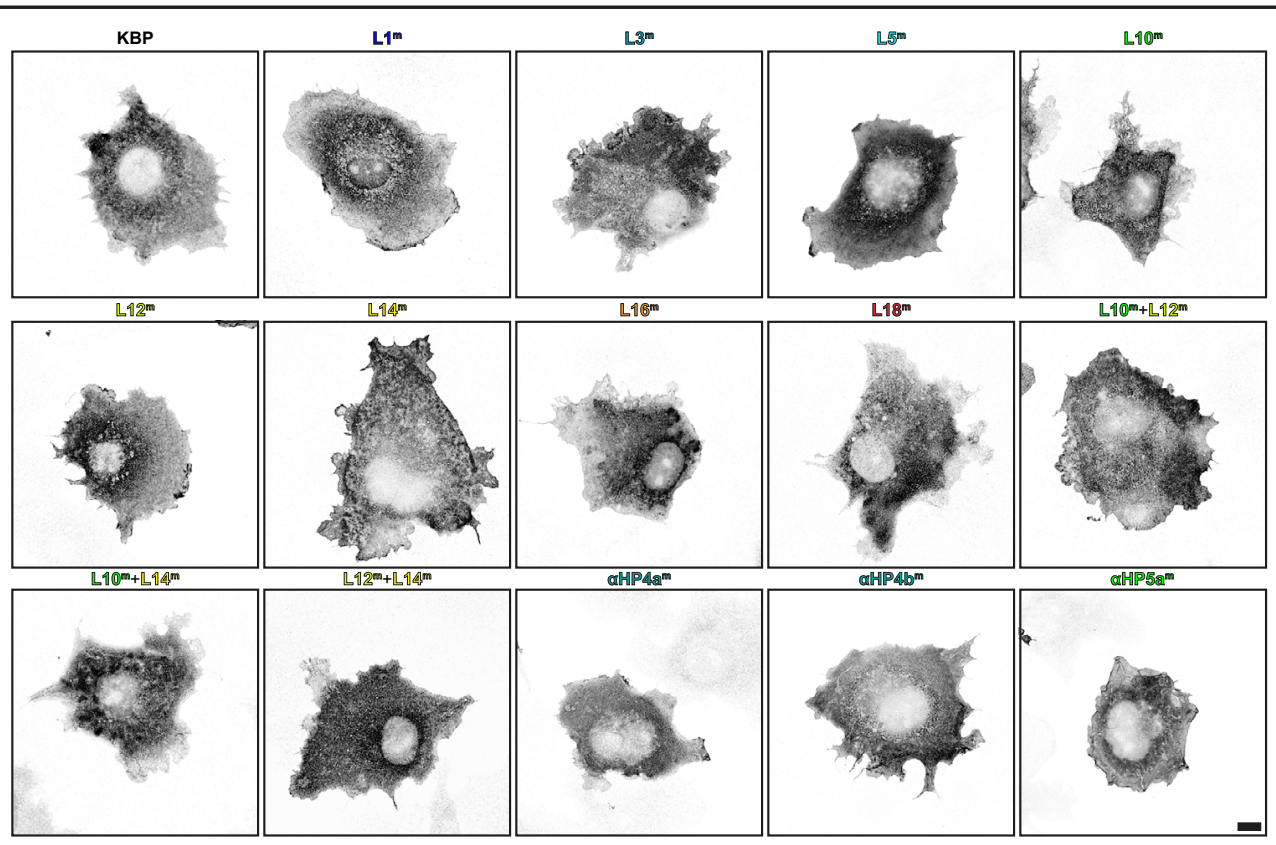

Figure 5-Supplement 1. Kinesin-binding protein (KBP) mutants show similar expression profiles in COS-7 cell Representative images of COS-7 cells expressing HA-KBP mutant constructs. Scale bar: $10 \mu \mathrm{m}$.

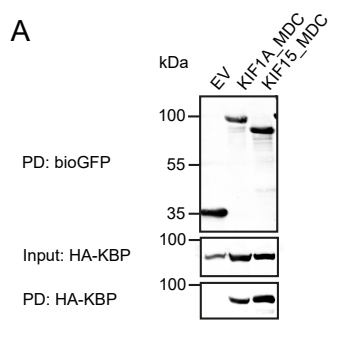

B

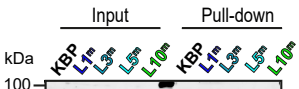

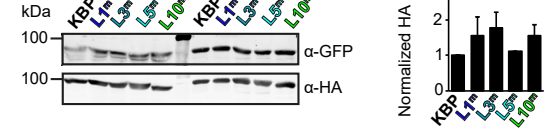
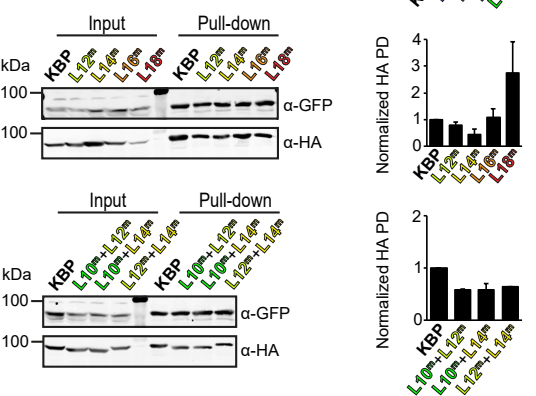

Input Pull-down
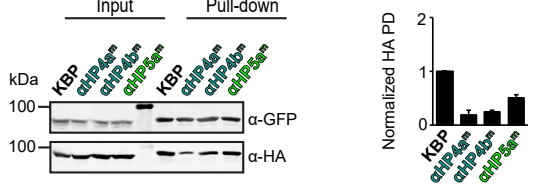

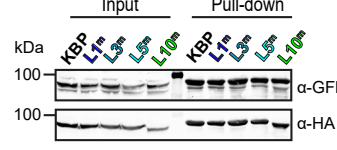
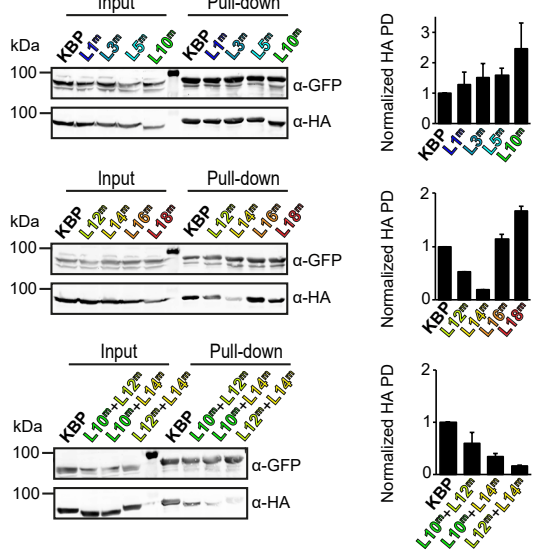

Input
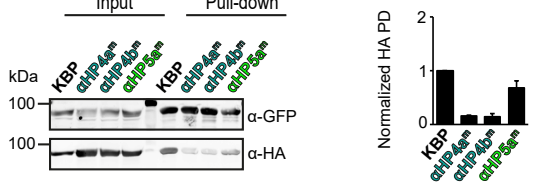

Figure 5-Supplement 2. Pull-down experiments demonstrate the effect of kinesin-binding protein (KBP) mutation on the interaction between KIFI5 and KIFIA

(A) Control pull-down experiment with bioGFP-EV, bioGFP-KIF1A_MDC or bioGFP-KIF15 MDC and HAKBP showing that KBP interacts with KIFIA_MDC and KIF15_MDC, but not with bioGFP-EV. (B,C) Example of pull-down experiments showing the interaction between (B) KIFIS_MDC or (C) KIFIA_MDC and mutated

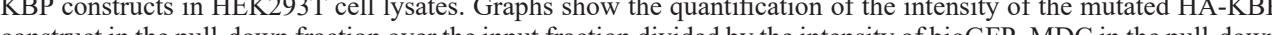
KB: constion fraction and nomalised to HA-KBP. Data ane displayed as meanıs.e.m. (daca from two independent experiments).
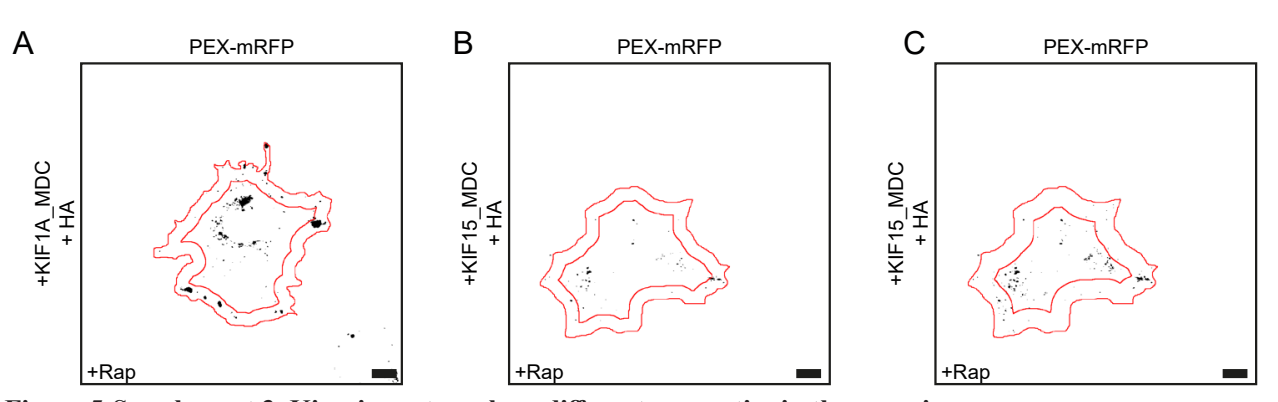

Figure 5-Supplement 3. Kinesin motors show different properties in the peroxisome assay (B,C) PEX-mRFP-FKBP and HA (left panels) with addition of rapalog. Images were thresholded at 10,000 (A,B) or $7500(\mathrm{C})$ and peripheral areas of $5 \mu \mathrm{m}(\mathrm{A}, \mathrm{B})$ or $7.5 \mu \mathrm{m}(\mathrm{C})$ are shown. Scale bars: $10 \mu \mathrm{m}$. 
Table 1. Cryo-electron microscopy reconstruction information and model refinement statistics and model geometry

Data collection, processing, and model refinement information for the kinesin-binding protein (KBP), KBP-KIF15 MD6S, and KIF15_MD6S-MT datasets.

\begin{tabular}{|c|c|c|c|}
\hline & $\begin{array}{l}\text { KBP } \\
\text { (EMDB: EMD-11338, }\end{array}$ & $\begin{array}{l}\text { KBP-KIF15_MD6S } \\
\text { (EMDB: EMD-11339 } \\
\text { PDB: 6PPH) }\end{array}$ & $\begin{array}{l}\text { KIF15_MD6S-MT } \\
\text { (EMDB̈: EMD-11340, } \\
\text { PDB: 6ZPD }\end{array}$ \\
\hline $\begin{array}{l}\text { Data collection and processing } \\
\text { Pixal size }(\S * *\end{array}$ & 10551043001047 & & 10D. (E) (1) \\
\hline $\begin{array}{l}\text { Number of micrographs (collected, final)* } \\
\text { Final particle number }\end{array}$ & $\begin{array}{l}9360,7547 \\
258,04981,628 \text { of which }\end{array}$ & 6497,5138 & 214,202 \\
\hline $\begin{array}{l}\text { Map resolution }(\AA) \\
\text { FSC threshold }{ }^{\dagger}\end{array}$ & $\begin{array}{l}\text { on graphene oxide) } \\
\text { I.6 } \\
0.143\end{array}$ & $\begin{array}{l}6.9 \\
\text { Independent half-map } \\
\text { FSC } 0.143\end{array}$ & $\begin{array}{l}4.5 \\
\text { Independent half-map } \\
\text { FSC } 0.143\end{array}$ \\
\hline Refinement & & & \\
\hline $\begin{array}{l}\text { Refinement resolution }(\AA) \\
\text { CC_mask: }\end{array}$ & $\begin{array}{l}4.6 \\
0.64\end{array}$ & $\begin{array}{l}6.9 \\
0.74\end{array}$ & $\begin{array}{l}6 \\
0.60\end{array}$ \\
\hline Map sharpening $B$-factor $\left(\AA^{2}\right)$ & -200 & -495 & -134 \\
\hline $\begin{array}{l}\text { Model composition } \\
\text { Nonhydrogen atoms }\end{array}$ & 3,808 & 6,232 & 9,420 \\
\hline $\begin{array}{l}\text { Protein residues } \\
\text { Ligands }\end{array}$ & ${ }_{0}^{610}$ & 1948 & ${ }_{4}^{1185}$ \\
\hline $\begin{array}{l}\text { R.m.s. deviations } \$ \\
\text { Bond lenoths }\end{array}$ & 001 & 010 & 000 \\
\hline Bond angles $\left(^{\circ}\right)$ & 0.96 & 1.07 & 0.17 \\
\hline $\begin{array}{l}\text { Valladaton" } \\
\text { MolProbity score }\end{array}$ & 1.66 & 1.84 & 1.95 \\
\hline $\begin{array}{l}\text { Clashscore } \\
\text { Poor rotamers }(0)\end{array}$ & 5.25 & 7.31 & 13.25 \\
\hline $\begin{array}{l}\text { Poor rotamers (\%) } \\
\text { Ramachandran plot" }\end{array}$ & & $0.9 \%$ & $0.1 \%$ \\
\hline Favored (\%) & 94.38 & 93.13 & 95.38 \\
\hline 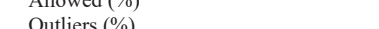 & 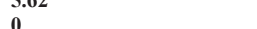 & 0 & \\
\hline
\end{tabular}

*Inclusive of all data collection sessions.

$\dagger$ The resolution value at the gold-standard Fourier Shell Correlation (FSC) 0.143 criterion between independently efined half-maps.

Cross-correlation provided by Phenix real-space refine (Afonine et al., 2018)

§Root-mean-square deviations of bond lengths or angles in the model.

Table 2. Kinesin-binding protein (KBP) mutants used in this study

The original and mutated amino acid (top) and nucleotide sequences (bottom) are shown for each construct.

\begin{tabular}{|c|c|c|}
\hline Construct & Original sequence & Mutated to \\
\hline L1 & EKEPYK & AAAPAA \\
\hline L3 & $\begin{array}{l}\text { gagaaggaaccatacaag } \\
\text { TEE }\end{array}$ & gcagcagcaccagcagca \\
\hline & acggaggag & gcaggagga \\
\hline L5 & $\begin{array}{l}\text { REE } \\
\text { REgagagaa }\end{array}$ & $\begin{array}{l}\text { AGA } \\
\text { gagagaca }\end{array}$ \\
\hline L10 & $\begin{array}{l}\text { agagaagaa } \\
\text { KISATEDTPEAEGEVEPEL } \\
\text { aagattcagccacacagagacactcotgaagctgaaggagaagtgccagagctt }\end{array}$ & $\begin{array}{l}\text { gagagaga } \\
\text { AGAGAGAGAGAGAGAGPGG } \\
\text { gcagagacagagagcagagacagagaccagcagagagcaggagcaggaccaggagag }\end{array}$ \\
\hline L12 & $\begin{array}{l}\text { aagatctcagccacagaagacactctgaagctgaaggagaagtgccagagctt } \\
\text { DGY }\end{array}$ & gcaggagcaggagcaggagcaggaccagcaggagcaggagcaggaccaggagga \\
\hline L14 & $\begin{array}{l}\text { gatggttat } \\
\text { DLNPQY }\end{array}$ & $\begin{array}{l}\text { ggcggggcg } \\
\text { AAGPAA }\end{array}$ \\
\hline L16 & $\begin{array}{l}\text { gacctgaatccacagatat } \\
\text { NKVPEEHIGEDVL }\end{array}$ & $\begin{array}{l}\text { gcagagagaccagcagac } \\
\text { AAGAPAAGAGAA }\end{array}$ \\
\hline L18 & $\begin{array}{l}\text { aataaagtattccetgagcatataggggaagatgttctt } \\
\text { EKHPE }\end{array}$ & $\begin{array}{l}\text { gcagcaggagcaccagcaggagcaggagcaggagcagca } \\
\text { AAGPG }\end{array}$ \\
\hline aHP4a & $\begin{array}{l}\text { gaaaagcatcctgag } \\
\text { YLcté } \\
\text { tacctagctcaa }\end{array}$ & $\begin{array}{l}\text { gagagaggaccagga } \\
\text { ALAAtagctgca } \\
\text { gcacta }\end{array}$ \\
\hline aHP4b & Q & A \\
\hline$\alpha \mathrm{HP} 5 \mathrm{a}$ & $\begin{array}{l}\text { lag } \\
\text { TLSQ } \\
\text { accttgtacag }\end{array}$ & $\begin{array}{l}\text { gca } \\
\text { ALSA } \\
\text { gcgttgtcagcg }\end{array}$ \\
\hline
\end{tabular}




\section{Inducible manipulation of motor-cargo interaction using engineered kinesin motors}

Jessica J. A. Hummel ${ }^{1}$, Casper C. Hoogenraad ${ }^{1,2}$

J. Cell Sci. (2021); 134: jes258776

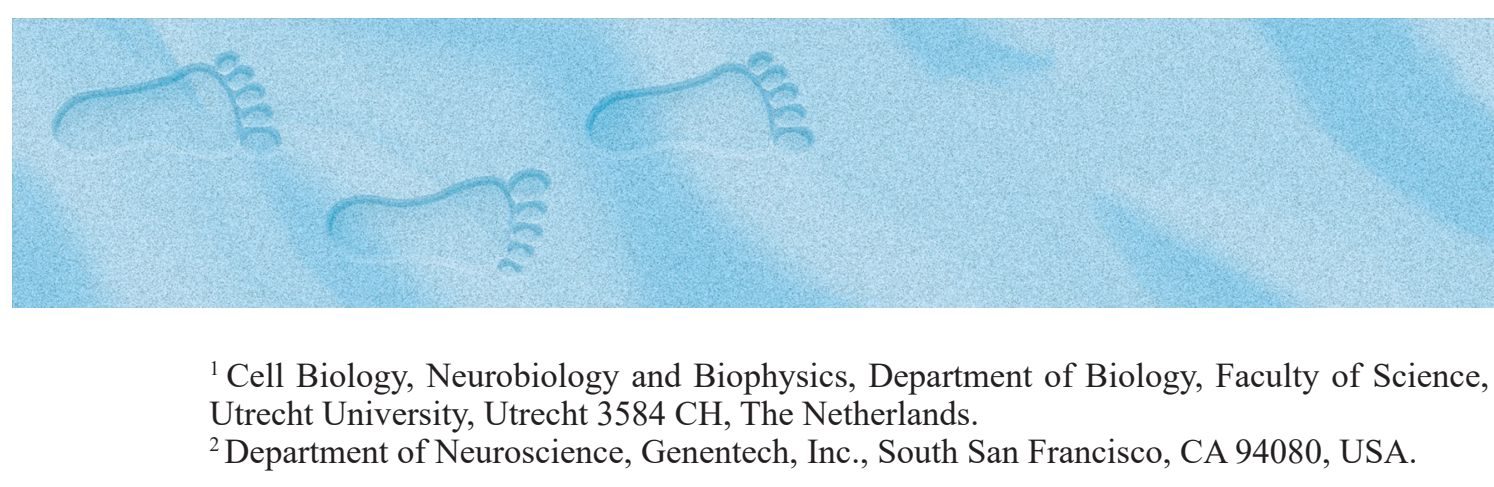




\section{ABSTRACT}

Molecular motors drive long-range intracellular transport of various vesicles and other cargoes within a cell. Identifying which kinesin motors interact with which type of transport

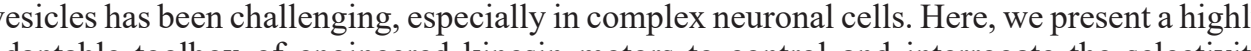
daptable toolbox of engineered kinesin motors to control and interrogate the selectivity and regulation of cargo transport with acute chemical induction. Selectivity of cargo-moto interaction can be addressed by systematic screening of a library of kinesin tails and neurona cargoes. Additionally, our toolbox can be used to study kinesin-cargo regulatory mechanisms, and we found that cargo trafficking by KIF16B is regulated by its PX domain. Furthermore, our toolbox enables acute manipulation of polarized trafficking in living neurons by steering transport into axons or dendrites. Engineering kinesin motors provides a powerful tool to map the specificity of interactions between kinesin and cargoes, manipulate polarized tran and investigate cargo-motor interaction modes.

\section{INTRODUCTION}

Intracellular transport of cargo, including protein complexes, organelles and mRNA, is of great importance for cell development and survival. This becomes especially clear in highly polarized cells, such as neurons, which rely on long-distance transport for their survival. In neurons, newly synthesized cargoes need to be transported from their site of synthesis, the cell body, to their destination in the axon and dendrites. Simultaneously, retrograde transport is required to remove aging proteins and organelles, and to enable retrograde signaling by cargoes such as neurotrophic factors (Maday et al., 2014). Defects in this long-distance cargoes such as neurotrophic factors (Maday et al., 2014). Defects in this long-distance
transport have been linked to many neurodegenerative diseases, including Alzheimer's disease and Parkinson's disease (Millecamps and Julien, 2013; Encalada and Goldstein, 2014). Neurons are highly polarized cells typically containing a single axon and multiple dendrites, which play different roles in electrochemical signaling. Axon and dendrites differ in their molecular composition and precise localization of proteins to the right domains is crucial for neuronal development and neuronal signaling (Bentley and Banker, 2016). din cells. Kinesins and dyneins use the microtubule cytoskeleton to facilitate long-distance transport across the cell, where kinesin motors move toward the microtubule plus-ends and dynein owards the microtubule minus-end (Hirokawa et al., 2010). Kinesin motors typically consis of a motor domain that uses the energy from ATP hydrolysis to walk along the microtubule and a tail domain that interacts with cargo (Hirokawa and Noda, 2008; Kato et al., 2018).So far, 45 different mammalian kinesin genes have been identifiedand classified into 14 families (Miki et al., 2001; Lawrence et al.,2004). The translocation patterns of different kinesins have been identified using constitutively active motors (Huang and Banker,2012; Yang et al., 2016) and by using an inducible dimerization system to link a constitutively active motor to (

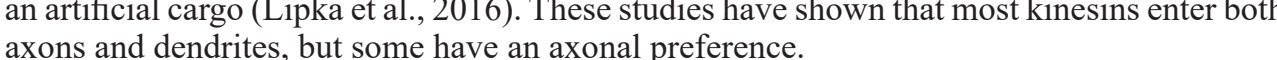
To ensure proper cargo localization, neurons have developed different cargo sorting pathways. Interestingly, it has been shown that dendritic cargo is restricted to dendrites an vesicles do not enter the axon (Burack et al., 2000; Farías et al., 2012). In contrast, axonal proteins are transported into both axons and dendrites, but cargo trafficking is biased to the axon (Burack et al., 2000; Nakataand Hirokawa, 2003). Tools that manipulate polarized cargo trafficking have provided more insights into the importance of proper cargo sorting for neuronal function. For example, by using nanoparticles and magnetic forces it has been shown that reversing endosomal trafficking affects neurite outgrowth and growth cone motility (Steketee et al., 2011). Furthermore, optogenetic tools that control mitochondrial and endosomal trafficking in neurons have shown that local positioning of recycling endosomes contributes to axon outgrowth (Van Bergeijk et al., 2015). However, these nanobiological and optogenetic tools are technically demanding and require optimization.
Therefore, general and easily adaptable tools to study and manipulate polarized cargo trafficking are of interest.

To solve the 'cargo problem', for example, addressing which cargo is transported by which kinesin (Terada and Hirokawa, 2000), different approaches have been used. Yeast two-hybrid and immunoprecipitation experiments have yielded potential kinesin-binding partners and identified adaptors that link kinesin motors and cargoes (Hirokawa and Noda, artners and identified adaptor 2008). However, the major limitation of these binding and biochemical assays is the lack of a native cellular environment. For example, the kinetics, thermodynamics, stoichiometry and cofactors, such as lipid membranes, that may affect the motor-cargo interaction are usually not present in these systems. Another approach is to disrupt kinesin motor function in cells by RNAi approaches and examine changes in cargo proteins, thereby providing information about the kinesin-cargo interaction (Hirokawa et al., 2010). The drawback of this approach has been the redundancy in the intracellular transport system. Often one cargo is transported by miltiple motors and cell drive cargo transport, and therefore false-negative results easily arise using these strategies. Recently, a split kinesin method was developed to identify kinesin motors that interact wit a fluorescently labeled cargo (Jenkins et al., 2012).Although providing an elegant method to identify kinesin-cargo interaction, the assay is limited by its dependence on single-cell imaging making the experiments time consuming and technically challenging.

Here, we used the principles of the split kinesin method (Jenkinset al., 2012) to develop a broad and highly adaptable toolbox of engineered kinesin motors to study motorcargo interactions using chemical induction, and we explored different applications to gain insights into the regulation of polarized transport in neurons. Using a systematic imaging platform in fixed neurons, we demonstrated several applications of the toolbox. First, we screened a library of kinesin tail domains for an interaction with one specific cargo.Second, we screened one specific tail domain for an interaction with different cargoes. Finally, we use the assay to further investigate the KIF16B interaction with both dense core vesicles (DCVs) and endosomal vesicles by using different tail domain fragments, point mutations and kinesin chimera constructs. In addition to the investigation of specific kinesin-cargo interactions, our toolbox enabled the manipulation of polarized trafficking of cargoes in living neurons and, by using the motor domains of KIF5C and KIF1A, we were able to successfully steer cargo into axona and dendritic tips. Our engineered motors provide an efficient and powerful tool for studying kinesin-cargo interactions in living neurons.

\section{RESULTS}

\section{Engineered motors to identify kinesin-cargo interactions}

We based the development of our engineered motor platform on the principle of the split kinesin method (Jenkins et al., 2012). Accordingly, we used the KIF5C motor domain (KIF5Cmd), which in neurons is constitutively active, traffics only into the axon and accumulates in distal axonal tips (Jacobson et al., 2006). Therefore, upon chemical linkage, the kinesin tail domain (KIFtd), which is unable to drive transport on its own, will be translocated into distal axonal tips. Adding a fluorescently labeled cargo to the system allows the identification of interactions between the KIFtd and cargo. In case of an interaction, a marked re-localization of the cargo into the axonal tips will be observed after addition of rapalog (Fig 1A). We of the cargo into the axonal tips will be observed after addition of rapalog (Fig 1A). We
first validated the approach using two previously reported interactions of KIF1A with DCV (Lo et al., 2011) and KIF13B with transferrin receptor (TfR)-containing vesicles (Jenkins et al., 2012). Therefore, we generated 3myc-tagged KIF1Atd and KIF13Btd constructs (Fig 1B) fused to an FKBP12-rapamycin-binding (FRB) domain. We tested the system by expressing KIF5Cmd, which was fused to an FKBP domain and mRFP, and either KIF1Atd two different conditions, one without rapalog and one where rapalog was added to the culture medium directly after transfection. Neurons were fixed 1 day after transfection and visualized by fluorescence microscopy 

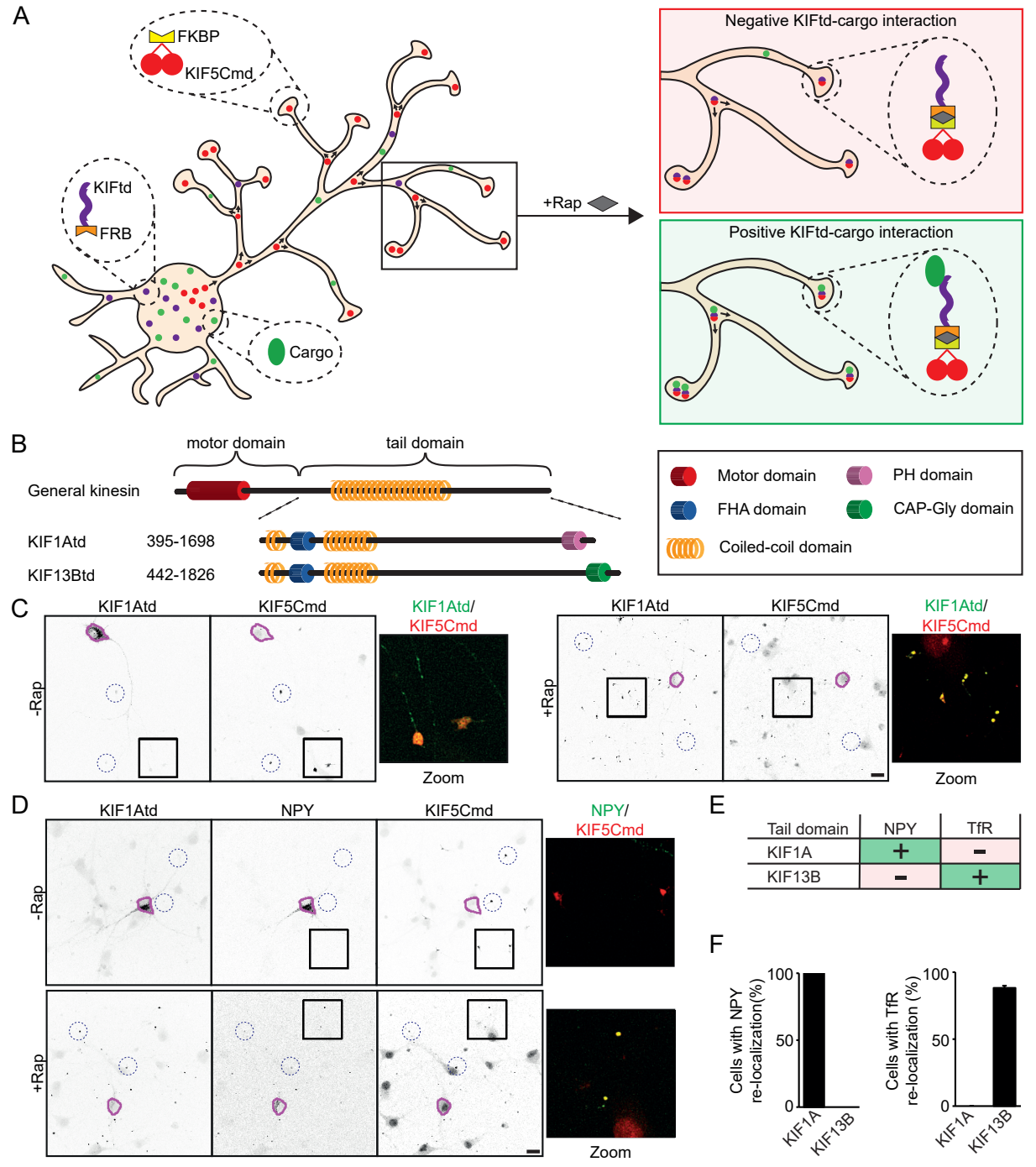

- Motor doma

(100) Coiled-coil dom

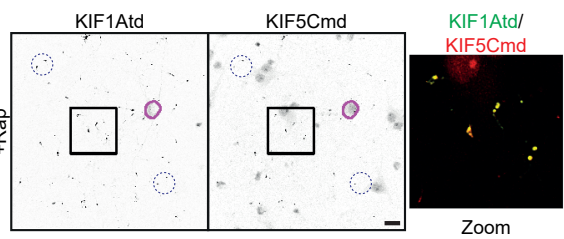

E
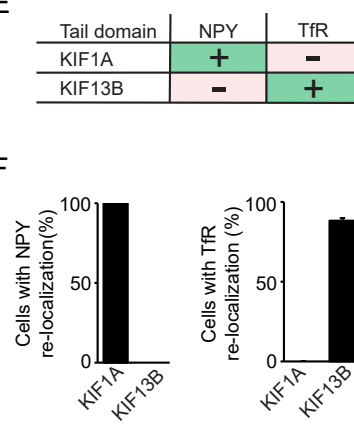

(A) Schematic representation of the engineered kinesin assay. Components of the assay include $\mathrm{KIF} 5 \mathrm{Cmd}$ fused to an FKBP domain, a kinesin tail domain fused to an FRB domain, and a fluorescently labeled cargo. Withou rapalog, the kinesin tail domain and cargo will be diffuse over the cell, whereas the KIF5Cmd will translocate in distal axonal tips. Addition of rapalog induces binding of the FKBP and FRB domains thereby generating a fully functional motor that re-localizes the kinesin tail domain into axonal tips. When there is no interaction between the kinesin tail and the cargo, the cargo will maintain its diffuse localization, whereas in case of an interaction the cargo (C) Representative images of hippocampal neurons co-expressing FKBP-mRFP-KIF5Cmd and FRB-3mycKIF1Atd without (left) or with (right) addition of $1 \mu \mathrm{M}$ rapalog at transfection. The purple line shows the outline of the cell soma and blue dotted circles indicate examples of axonal tips. A merged magnified view of the boxed region is shown. (D) Representative images of hippocampal neurons co-expressing FKBP-mRFP-KIF5Cmd, FRB-3mycKIF1Atd and NPY-GFP without (top) or with (bottom) addition of $1 \mu \mathrm{M}$ rapalog at transfection. The purple line shows the outline of the cell soma and blue dotted circles indicate examples of axonal tips. A merged magnified view of the boxed regions is shown. (E) Overview of the identified interactions between DCVs or endosomal vesicles and KIFAAtd or KIF SBd in the engineered kinesin assay. (F) Quantification of the percentage of cells in whic mean \pm s.e $\mathrm{m}$. ( $\mathrm{N}=2$ independent experiments, $\mathrm{n}=60$ cells). Scale bars: $20 \mu \mathrm{m}$.
In the absence of rapalog, KIF5Cmd clearly accumulated in distal tips, whereas the KIF1A and KIF13B tails were diffuse over the cell. Upon rapalog treatment the KIF tail domain re-localized into distal axonal tips where they colocalized with KIF5Cmd, verifying that rapalog induced binding between KIF5Cmd and KIFtd, thereby generating a fully functional and non-auto-inhibited kinesin motor (Fig 1C; Fig S1A). Next, we verified the interaction of these kinesin tails with GFP-labeled cargo. Cultured hippocampal neurons were transfected with KIF5Cmd, KIFtd, and GFP-tagged Neuropeptide Y (NPY) to label DCVs or GFPtagged TfR to label endosomal vesicles and treated with or without rapalog. Upon rapalog treatment, we observed a clear re-localization of cargo into distal tips, where it colocalized with KIF5Cmd and KIFtd, and we conclude that rapalog induced transport of NPY-positive DCVs by KIF1Atd and transport of TfR containing endosomal vesicles by KIF13Btd (Fig 1D; Fig S1B). In contrast, KIF13Btd was not able to re-localize NPY vesicles and there was no TfR re-localization by KIF1A, thereby providing negative controls (Fig S1C,D). Overall, these data show that the engineered kinesi between kinesins and their cargo in fixed hippocampal neurons.

\section{Evaluation of kinesin-cargo interactions in fixed neurons}

The original split kinesin method used live-imaging as the main approach to quantify kinesin-cargo interaction. As single-cell live imaging is a time consuming and technically challenging process, we explored different ways to quantify kinesin-cargo interaction in fixed neurons. As shown in Fig 1, treating the cells with rapalog directly after transfection and fixation after $24 \mathrm{~h}$ enabled visualization of KIFtd and cargo re-localization in the cases where there was kinesin-cargo interaction. Therefore, one could simply visualize fixed coverslips and classify them as positive for interaction when colocalization of KIF5Cmd, KIFtd and cargo in distal tips is observed in the rapalog-treated condition, or negative for interaction when no re-localization of the cargo is observed after rapalog treatment. This was undertaken for DCV and TfR transport by KIF1 Atd and KIF13Btd (Fig 1E). However, more quantitative measurements of the efficiency of induced kinesin-cargo binding might be required for some research purposes, and therefore we developed two methods to further quantify kinesin-cargo interactions. In the first method, coverslips are screened for neurons that express all three constructs, KIF5Cmd, KIFtd and GFP-cargo. A neuron is classified as positive for interaction when all three constructs colocalize in distal tips or negative when this is not the case. The percentage of cells in which cargo transport by the KIFtd is observed can then be calculated as is shown for DCV and TfR transport by KIF1Atd and KIF13Btd (Fig 1F). A second method for quantification was based on the cargo intensity in distal tips and soma. In case of a positive tail-cargo interaction, cargo is moved out of the soma into neurons expressing KIF5Cmd, KIF1Atd and NPY-GFP, either treated or not treated with rapalog were taken, and five random axonal tips and the cell soma were selected based on the fill (Fig 2A). Next, cargo intensity in these regions of interest was measured and the ratio of cargo intensity in the distal tips over cargo intensity in the soma was calculated (Fig 2B) Normalization to the condition without rapalog treatment, shows that, in the case of kinesincargo binding, treatment with rapalog results in a higher ratio of cargo intensity in distal tips to that in the soma ( $\mathrm{Fig} 2 \mathrm{C}$ ), thereby providing a measure for kinesin-cargo binding. In contrast, neurons expressing changes between the ratio of cargo intensity in distal axonal tips to that in the cell soma (Fig S2A-C), thereby showing that this method quantitatively identifies positive and negative kinesin-cargo interactions. In conclusion, the assay allows both qualitative and quantitative methods to evaluate kinesin-cargo interactions in fixed neurons. 

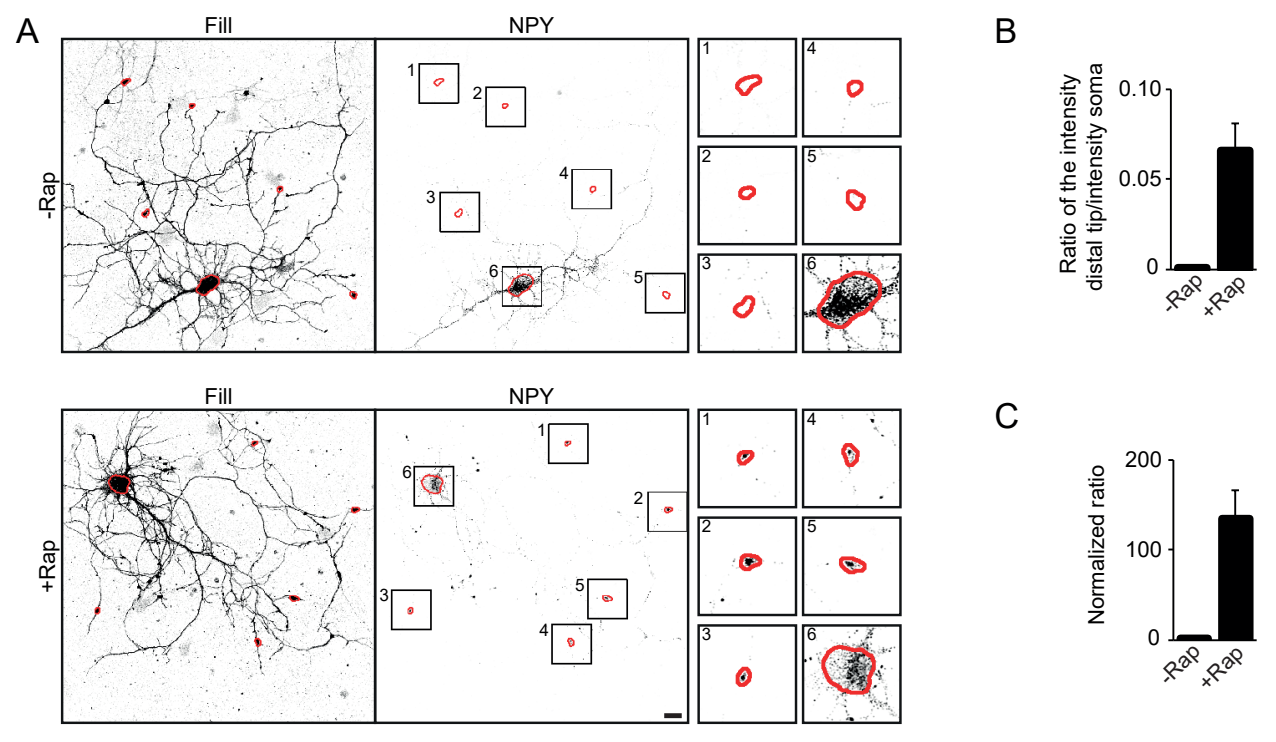

C

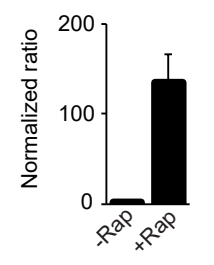

$\mathrm{D}$
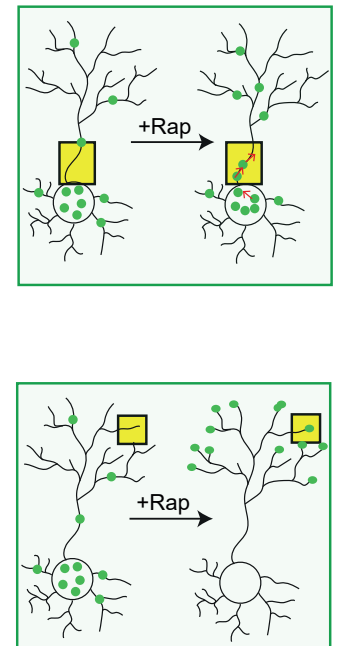

$\mathrm{E}$
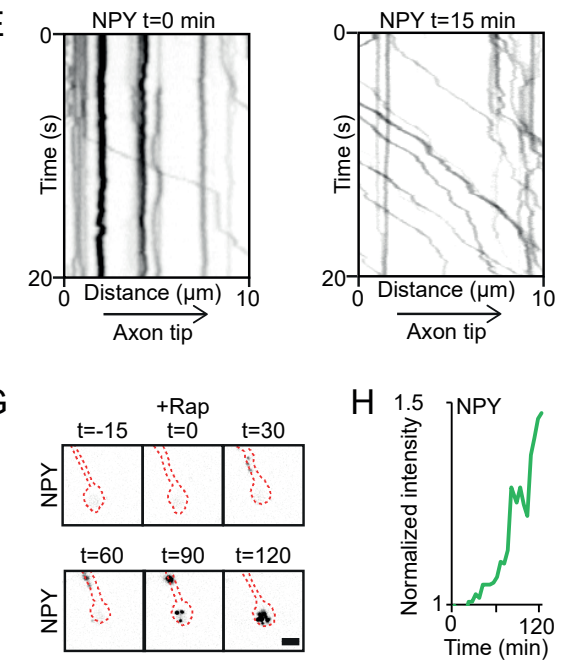

H 1.5 NPY

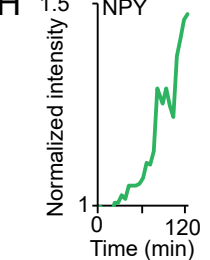

Figure 2. Quantification of kinesin-cargo binding

(A) Representative example of the quantification method of the ratio of cargo intensity in distal tips over the soma in neurons co-expressing FKBP-mRFP-KIF5Cmd, FRB-3myc-KIF1Atd and NPY-GFP. Five random distal tips and the soma are selected from the fill (HA- $\beta$-galoctidase) for both the conditions without (top) or with (bottom) rapalog
(outlined in red). The same regions of interest are then selected in the cargo channel and cargo intensity in these regiog is measured. Magnified views of the regions of interest are shown in the small right panels. (B, C) Quantifications of the ratio of cargo intensity in the distal tips to the intensity in the cell soma (B) and the ratio when normalized to the condition without rapalog (C) of the example neuron depicted in (A). Results are mean \pm s.e.m. ( $\mathrm{n}=1$ cell). (D) Schematic depiction of the readout of the engineered kinesin assay in a neuron. Yellow boxes indicate the localization for live-cell imaging in the axon initial segment. (E) Representative kymographs showing movement of NPY vesicles in the axon initial segment before addition of rapalog (left) and $15 \mathrm{~min}$ after (right) addition of $1 \mu \mathrm{M}$ rapalog in neurons co-expressing FKBP-mRFP-KIF5Cmd, FRB-3myc-KIF1Atd and NPY-GFP. (F) Schematic depiction of the readout of the assay in a neuron. Yellow boxes indicate the localization for live-cell imaging in the growth cone. (G) Stills showing the accumulation of NPY in an axonal tip (dotted red line) over time (in minutes) after addition of rapalog in neurons co-expressing FKBP-mRFP-KIF5Cmd, FRB-3myc-KIF1Atd and NPY-GFP. (H) Graph showing
the intensity profile of NPY in the axonal tip (shown in G) after addition of rapalog. Scale bars: $20 \mu \mathrm{m}(\mathrm{A}), 2 \mu \mathrm{m}(\mathrm{G})$.

Assessment of kinesin-cargo interactions by live-cell imaging

In addition to the evaluation of kinesin-cargo interaction in fixed cells, we were also interested in exploring live-cell imaging approaches. Therefore, we designed two approaches to assess motor-cargo interactions by live-cell imaging and applied these to visualize the interaction between KIF1A and DCVs. In the first approach, which is similar to the approach used in the original split kinesin method, we imaged the axon initial segment. In case of an interaction between kinesin and cargo an increase in vesicle entry into the axon is expected upon addition of rapalog (Fig 2D). In neurons expressing KIF5Cmd, GFP-NPY and KIF1Atd we observed an increased axonal vesicle entry (Fig 2E; Movie 1). In the second approach we imaged an axonal tip over time. In case of association with the KIFtd we expect an accumulation of a labeled vesicle population in the tip (Fig 2F). When imaging hippocampal neurons expressing KIF5Cmd, GFP-NPY and KIF1 Atd we indeed observed accumulation of NPY in the axonal tip $\sim 90$ min after addition of rapalog (Fig 2G H; Movie 2). Together, these data show that live-imaging provides an effective way to visualize kinesin-cargo interactions in hippocampal neurons.

\section{Systematic screening to identify which kinesin binds to a cargo of interest}

After developing a variety of analysis methods, we further demonstrate that our toolbox can be used to gather more insights on kinesin-cargo interactions. First, we screened for kinesin-tail interactions with one specific cargo of interest. We generated a library of kinesin tail domain constructs (fused to FRB and tagged with 3 myc) in which we included the tail domains of the 16 transporting kinesins from the kinesin-1, kinesin-2, kinesin-3 and kinesin-4 families (Fig 3A) (Lipka et al., 2016), thereby expanding on the original split kinesin method. Expression of the kinesin tail domains in neurons showed that most tails have a diffuse localization across the neurons, but some, for example, KIF1Atd, KIF1Ctd and KIF16Btd have a clear vesicular-like expression pattern (Fig S3A). We then tested the rapalog-induced binding for each kinesin tail (examples with KIF5Ctd, KIF17td, KIF16Btd and KIF21Btd are shown in Fig S3B). Some kinesin tails, for example, KIF5Ctd, were found to be slightly enriched in axonal tips without addition of rapalog. However, these tail domains still showed a large diffuse pool across the cell, and addition of rapalog induced a clear change in the tail domain localization out of the cell soma into distal axonal tips (Fig S3B). We next wanted to identify all kinesin tails that can interact with DCVs and TfRcontaining vesicles and screened our library of tail domains in the assay. Analysis in fixed neurons and quantification of the percentage of cells that showed cargo transport by a KIFtd was the most suitable approach for analyzing such large library screens, and we used this method for analysis of all further experiments. Therefore, fixed neurons were visualized by fluorescence microscopy and classified as positive or negative for interaction. Systematic analysis of all tail domains and both cargoes resulted in an overview of all kinesins that associate with DCVs and TfR vesicles. We identified that DCVs associate with KIF1Atd, KIF1Bßtd and KIF16Btd, whereas TfR vesicles are transported by KIF13Atd, KIF13Btd, KIF16Btd and KIF4Btd (Fig 3B). For all positive interactions, we quantified the percentage of cells in which kinesin-cargo interaction was observed. For DCVs, transport by KIF1Atd was seen in all cells, whereas $\sim 80 \%$ of the cells showed re-localization by KIF1B $\beta$ td and KIF16Btd, suggesting a slightly weaker interaction between these kinesin tails and DCVs (Fig 3C). TfR vesicles were very efficiently re-localized by KIF13Btd and KIF16Btd, as, respectively, $90 \%$ and $100 \%$ of cells showed positive interaction. In contrast for KIF13Atd and and KIF two motors have lower binding affinity for TfR vesicles (Fig 3D). These results show that
the assay provides a robust screening platform to identify kinesin interactions with a specific cargo. 

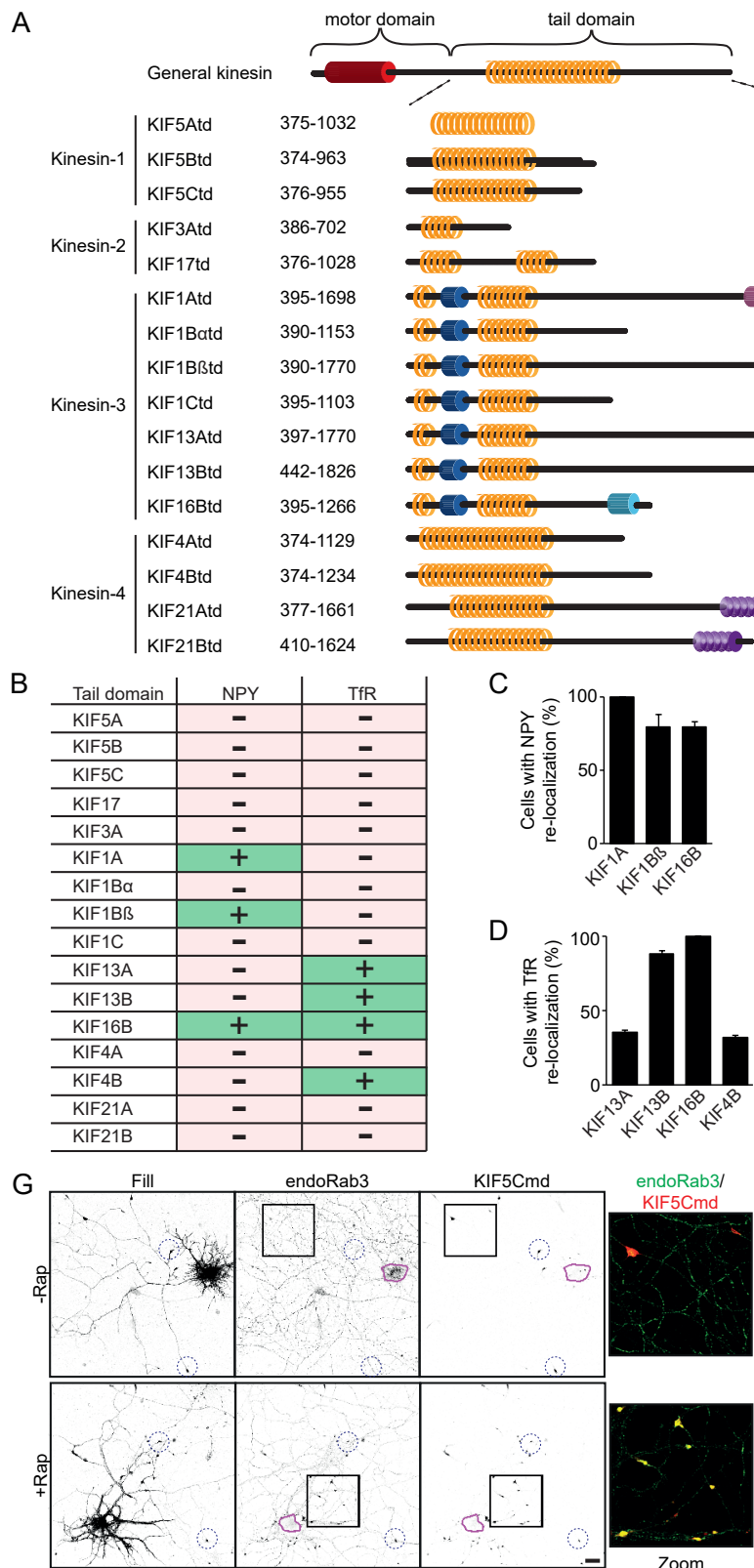

igure 3. Screening applications of the engineered kinesin platform

(A) Overview of the library of kinesin tail domains used to screen for cargo interactions. (B) Overview of the identified kinesins that interact with DCVs and TfR vesicles in the engineered kinesin assay screen. (C,D) Quantification of the percentage of cells in which NPY vesicles $(C)$ or TfR vesicles (D) are re-localized to distal axonal tips in the assay by the
identified interacting motors. Results are mean \pm s.e.m. $(\mathrm{N}=2$ independent experiments, $n=50-60$ cells). (E) Overview of the identified cargoes that interact with KIF1 Atd in the engineered kinesin assay screen. (F) Quantification of the percentage of cells in which cargo vesicles are re-localized to distal axonal tips by KIF1Atd in the assay. Results are mean \pm s.e.m. (error $=0)(\mathrm{N}=2$ independent experiments, $\mathrm{n}=60$ cells). (G) Representative images of a hippocampal neuron co-expressing FKBP-mRFP-KIF5Cmd and FRB-3myc-KIF1Atd and immunostained for Rab3 without (top) or with (bottom) addition of $1 \mu \mathrm{M}$ rapalog. The purple line shows the outline of the cell soma and blue dotted circles indicate examples of axonal tips. A merged magnified view of the boxed regions is shown. Scale bar: $20 \mu \mathrm{m}$.
Systematic screening to identify which kinesin of interest binds to a variety of cargoes

The engineered kinesin motor assay can also be used to screen one kinesin tail domain for an interaction with a variety of organelles, vesicles and other cargoes. Here, we have used TfR-containing vesicles, synaptic vesicles (SVs, visualized using Rab3 A-GFP), lysosomes (visualized using GFP-LAMP1), and mitochondria (visualized using mito-GFP). This screen showed that KIF1Atd interacts with DCVs, SVs and lysosomes, whereas no interaction was observed with TfR vesicles or mitochondria (Fig 3E,F). In addition to using the assay with overexpressed marker proteins, we were also able to visualize the association of KIF1Atd with endogenous SVs by immunostaining for Rab3A (Fig 3G). These results show that the engineered kinesin motor assay is a robust tool to identify motor-cargo interactions.

Identification of the KIF16B PX domain in vesicle association

We next determined whether our engineered motor platform can be used to develop better mechanistic insight into specific kinesin-cargo interactions. Therefore, we turned our attention to KIF16Btd, which, in our screens, was found to interact both with DCVs and TfR-containing endosomal vesicles. Previously, KIF16B has been implicated in endosomal trafficking and it was found that its PX domain, and specifically L1197, is crucial for the interaction of KIF16B with early endosomes (Farkhondeh et al., 2015). To assess the involvement of the PX domain and L1197 in vesicle association, we generated constructs containing truncated and mutated KIF16Btd fragments linked to FRB and 3myc (Fig 4A). Expression of these constructs showed that removal of the PX or introducing the L1197F mutation resulted in loss of the KIF16Btd vesicular expression pattern (Fig S4A). We then screened these KIF16Btd constructs for an interaction with DCVs and TfR endosomal vesicles. Constructs in which the PX domain of KIF16Btd was removed could not associate with either NPY or TfR vesicles, whereas a construct containing only the PX domain was able to interact with both DCVs and endosomal vesicles (Fig 4B-E; Fig S1B). specific residue in the PX domain is crucial for vesicle association (Fig 4B). We further teste whether the PX domain of KIF16B is involved in vesicle binding by generating KIF1A-KIF16B chimeras in which the PH domain of KIF1A and the PX domain of KIF16B are swapped (Fig 4F). Localization of these constructs showed that swapping the PX domain of KIF16B for the PH domain of KIF1A (KIF16B-PH) resulted in loss of the KIF16B vesicular localization. Interestingly, when the PH domain of KIF1A was replaced by the PX domain (KIF1A-PX) the KIF1Atd adopted the localization of the PX domain (Fig S4C). As expected, KIF16B-PH was not able to transport NPY or TfR in the assay. However, KIF1A-PX could transport both DCVs and endosomal vesicles (Fig 4G-J; Fig S4D). These results suggest that the PX domain of KIF16B is sufficient for the interaction with DCVs and endosomal vesicles, and provides strong evidence of how the engineered motor platform can help to identify specific kinesin tail domain regions critical for cargo binding.

\section{Different motor domains transport cargo to axon and dendrites}

Having shown several applications of our engineered motor platform in the identification of kinesin-cargo interaction, we then wondered whether we could use our approach to artificially induce the targeting of cargoes to other neuronal compartments, such as dendrites or the cell body. To do so, we used the motor domains of KIF1A (KIF1Amd), KIF1C (KIF1Cmd) and KIF13B (KIF13Bmd), which move in both axons and dendrites. Additionally, we used the KIF6 motor domain (KIF6md), which is a non-moving motor (Lipka et al., 2016), to investigate whether we could capture a cargo in the cell soma. We fused the motor domain hippocam, KIFIC, KIFl3B and KII tips, although there was also some motor present along dendrites and axons and in the cell body. 
A

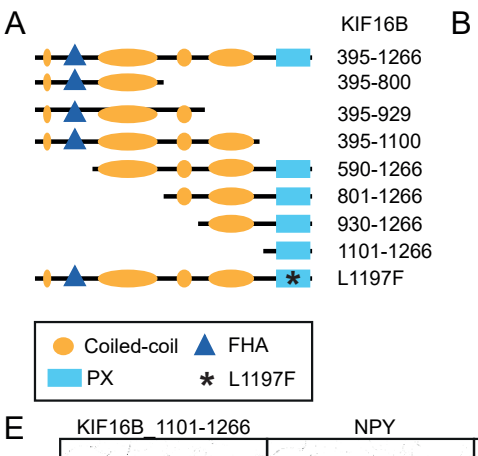

C

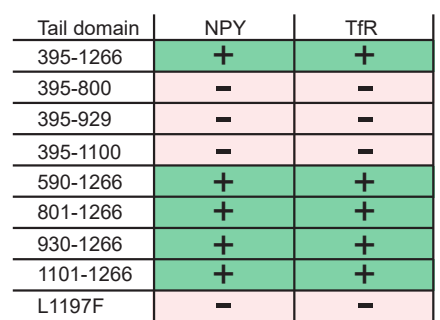

E

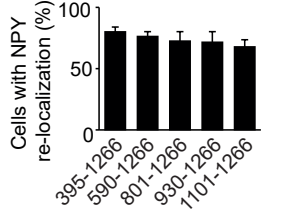

D

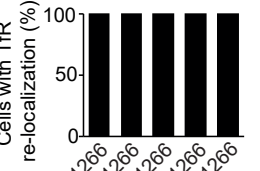

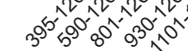

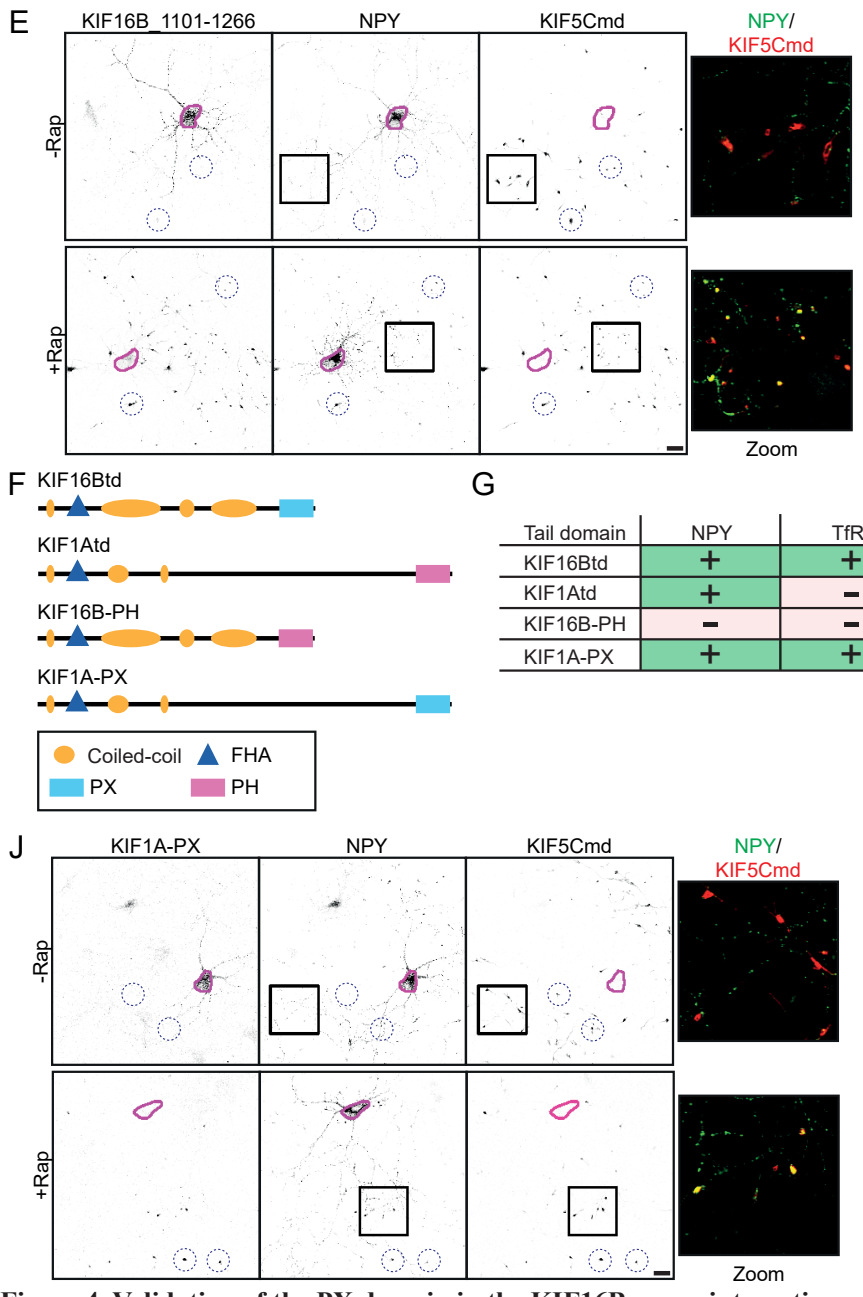

Figure 4. Validation of the PX domain in the KIF16B-cargo interaction

(A) Schematic representation of KIF16Btd, truncated KIF16Btd fragments and mutated KIF16Btd. (B) Overview of the different KIF16Btd constructs and their interaction with DCVs and TfR vesicles in the engineered kinesi assay screen. (C,D) Quantification of the percentage of cells in which NPY vesicles (C) or TfR vesicles (D) are re-localized to distal axonal tips in the assay with the identified interacting KIF16Btd fragments. Results are mean \pm s.e.m. (error $=0$ in $\mathrm{D})(\mathrm{N}=2$ independent experiments, $\mathrm{n}=60$ cells). (E) Representative images of hippocampal neurons co-expressing FKBP-mRFP-KIF5Cmd, FRB-3myc-KIF16B_1101-1266 and NPY-GFP without (top) or with (bottom) addition of $1 \mu \mathrm{M}$ rapalog at transfection. The purple line shows the outline of the cell soma and
blue dotted circles indicate examples of axonal tips. A merged magnified view of the boxed regions is shown

(F) Schematic representation of KIF16Btd, KIF1Atd, KIF16B-PH and KIF1A-PX. (G) Overview of KIF16Btd, KIFIAtd and KIH assay screen. (H,I) Quantification of the percentage of cells in which NPY vesicles (H) or TfR vesicles (I) are re-localized to distal axonal tips in the assay by KIF16Btd, KIF1Atd, and KIF16B-KIF1A chimeras. Results are neurons co-expressing FKBP-mRFP-KIF5Cmd, FRB-3myc-KIF1A-PX and NPY-GFP without (top) or with (bottom) addition of $1 \mu \mathrm{M}$ rapalog at transfection. The purple line shows the outline of the cell soma and blue dotted 作

KIF1Cmd localized in the cell body and along dendrites and axons, but we did not observe clear accumulation of the motor in distal tips. The KIF13Bmd localized in the somatodendritic region, without accumulation of the motor in distal tips. Finally, KIF6m was localized mainly around the cell body (Fig 5A,B). Next, we co-expressed KIF1 Amd, KIF1Cmd, KIF13Bmd and KIF6md together with KIF1Atd and GFP-NPY, with or without rapalog treatment, and visualized NPY re-localization. Using KIF1Amd, we observed DCV re-localization into both axonal and dendritic tips in all cells (Fig 5C; Fig S5B,C). Before addition of rapalog, KIF1Cmd was localized across the cell with no specific accumulation Interestingly, after rapalog addition and generation of a full motor that interacts with DCVs, KIF1Cmd translocated and accumulated into distal axonal and dendritic tips, suggesting that binding to a tail domain and cargo relieves KIF1Cmd autoinhibition (Fig S5A-C). When using KIF13Bmd, no clear NPY re-localization was observed, as the motor domain, tail domain and cargo colocalized, but were spread across the cell. We did observe that KIF13Bm whereas the motor domain was mainly somatodendritic in absence of rapalog (Fig S5D). These observations with KIF1Cmd and KIF13Bmd suggest an additional layer of regulation and/or motor activity when coupled to a kinesin tail and cargo. Using KIF6md in the assay led to a large accumulation of the motor with cargo in the cell soma (Fig S5E). Together, these results show that inducing the coupling of a given cargo to different kinesin motor domains can be used to control its localization in a specific neuronal compartment.

\section{Engineered kinesin motors override polarized cargo trafficking in neurons}

Using the KIF5Cmd we showed that we were able to re-localize dendritic TfR vesicles into the axon (Fig S1B). As the KIF1Amd was able to translocate cargo into both axons and dendrites (Fig 5B), we were wondering whether we could use the engineered kinesin assay to re-localize axonal cargo into dendrites. Therefore, we analyzed the distribution of SVs, which interact with KIF1Atd in our engineered motor assay, and mitochondria, which interact with KIF1B $\alpha$ (Nangaku et al., 1994). We expressed KIF1Amd together with either GFP-Rab3 and KIF1Atd, or mito-GFP and KIF1Batd in hippocampal neurons and subjected these neurons to rapalog treatment. Interestingly, we observed re-localization of both SVs and mitochondria into dendritic tips by KIF1Amd when coupled to KIF1Atd or KIF1Batd, respectively (Fig 5D; Fig S5F,G). Thus, using engineered kinesin motor cargoes can be re-localized into other neuronal compartments and reverse the polarized cargo sorting in cultured neurons.

\section{DISCUSSION}

Engineered kinesins allow rapid screening of motor cargo interactions

Cargo-translocation assays have previously been used to study cargo trafficking in neurons (Jenkins et al., 2012; Lipka et al., 2016). However, these assays are limited by their dependence on live-cell imaging, making the experiments technically challenging and time consuming. Here, we developed several methods to visualize and quantify kinesin-cargo interactions in hippocampal neurons by live-cell imaging as well as in fixed cells. We showed that positive cargo interactions result in a clear re-localization of vesicles into axonal tips, which provides an easily interpretable readout in fixed cells and allows systematic detection of experiments, as were mainly used in the original split kinesin method (Jenkins et al., 2012). 
A
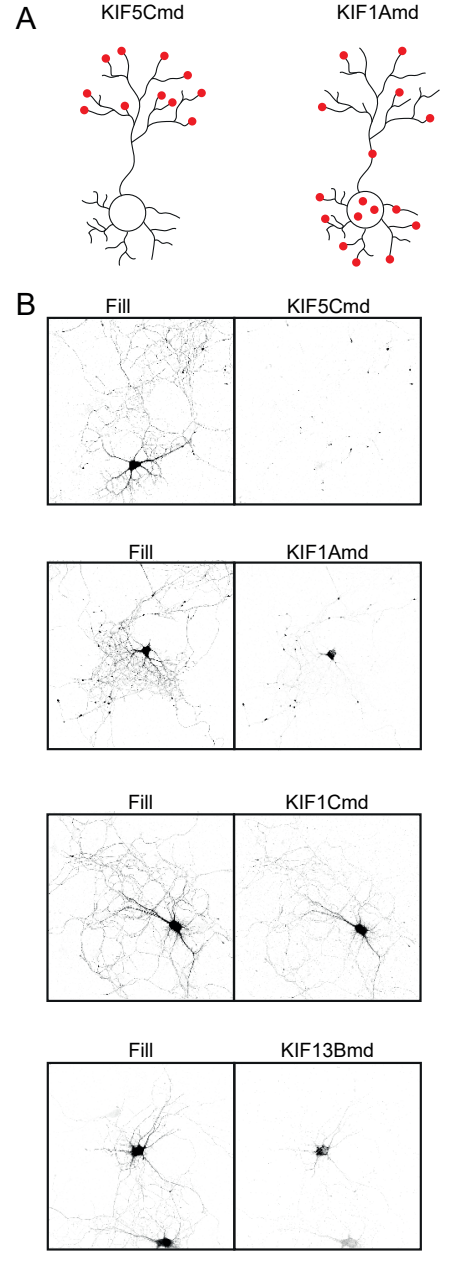

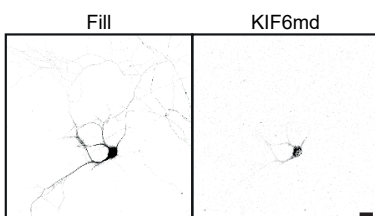

KIF1Cmd

2) 20

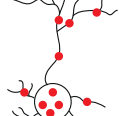

(2)-

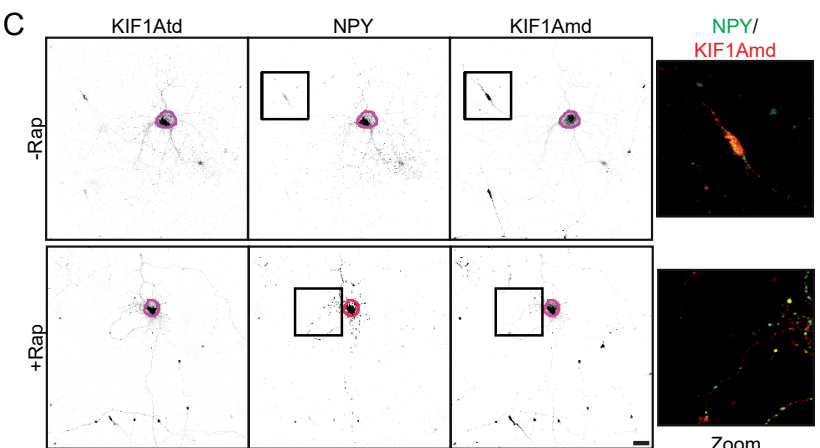

D
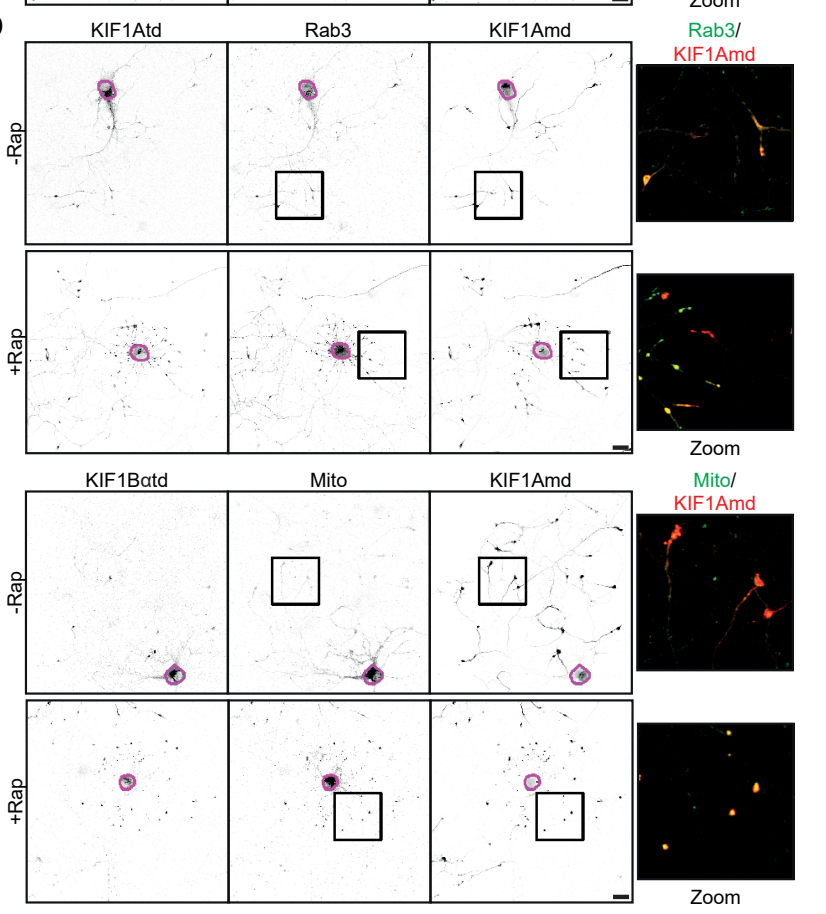

Figure 5. Different kinesin motor domains drive axonal and dendritic transport

(A) Schematic representations of the localization of the different motor domains in neurons. (B) Representative KIF1Amd, FKBP-mRFP-KIF1Cmd, FKBP-mRFP-KIF13Bmd or FKBP-mRFP-KIF6md (C) Representative images of hippocampal neurons co-expressing FKBP-mRFP-KIF1Amd, FRB-3myc-KIF1Atd and NPY-GFP without (top) or with (bottom) addition of $1 \mu \mathrm{M}$ rapalog at transfection. The purple line shows the outline of the cell soma, and a merged magnified view of the boxed regions is shown. (D) Representative images of hippocampal neurons co-expressing FKBP-mRFP-KIF1Amd together with FRB-3myc-KIF1Atd and Rab3-GFP or FRB-3mycKIF1Batd and Mito-GFP without (top) or with (bottom) addition of $1 \mu \mathrm{M}$ rapalog at transfection. The purple line shows the outline of the cell soma, and a merged magnified view of the boxed regions is shown. Scale bars: $20 \mu \mathrm{m}$.
Furthermore, the fixed approach used in the original method to quantify TfR re-localization from dendrites to the axon depends on a cargo being mainly dendritic before rapalog addition, thereby limiting this analysis method to specific cargo. Thus, our optimized analysis methods expand on the original assay and provide an easily adaptable platform to study a wide variety of thinesin-crgo interaction. In this study, we demonstrated several applications of

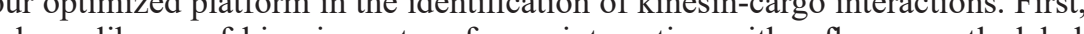
a large library of kinesin motors for an interaction with a fluorescently labeled cargo and identified all kinesins that interact with DCVs and TfR-containing endosomal vesicles. Consistent with previous findings, we found interactions of KIF1A with DCVs (Lo et al., 2011) and KIF13B with TfR-containing endosomal vesicles (Jenkins et al., 2012), thereby confirming that the assay successfully identifies known kinesin-cargo associations. Within our laboratory we have also successfully used the platform to identify kinesin interactions with the TrkB receptor (Zahavi et al., 2021). Furthermore, we showed that the engineered kinesin assay can be used to identify interactions of a specific tail domain and a library of For inster. we idrif or instance, we identifed DCVs, SVs and lysosomes as cargo for KIFlA, whereas no interaction was found with TfR-containing endosomes or mitochondria. We were also able to visualize the interaction of KIF1Atd with endogenous SVs by immunostaining with antiRab3 antibodies. In our screening, we found that KIF16B associates with both DCVs and TfR vesicles, and we further investigated the KIF16B-cargo interaction using different truncated and mutated KIF16B fragments and KIF1A-KIF16B chimeras in the engineered kinesin assay. This revealed that the PX domain, and specifically L1197 within the PX domain, is required for cargo binding, which is in line wit Overall, we show that the engineered kinesin assay provides a robust tool to identify, quantify and study kinesin-cargo interactions.

\section{Re-localizing polarized cargo into specific neuronal compartments}

Precise delivery of proteins into the right neuronal compartment is crucial for neuronal development and function (Bentley and Banker, 2016). Manipulation of the polarized trafficking machinery using nanobiology or optogenetic tools has provided insights into the role of endosomal trafficking in neuronal function (Steketee et al., 2011; Van Bergeijk et al., 2015). However, such techniques require specific technology and optimization, making them difficult to implement. Here, we show that our engineered motor platform enables the selective transport of cargo vesicles upon chemical induction. Our engineered kinesins can re-localize cargo into other neuronal compartments, overriding their endogenous polarized sorting machinery. We show that dendritic TfR-containing vesicles could be re-localized into the KIF1A motor domain Therefore, engineered motors provide an efficient and easy approach to manipulate polarized cargo trafficking. In addition, our toolbox also provides a platform to further study motor function. For example, we observed that the KIF1C motor domain accumulated in distal axonal and dendritic tips only upon chemically induced formation of a full functional motor that interacts with DCVs, suggesting that cargo binding provides an additional layer of regulation of motor activity, potentially by relieving auto-inhibition. Furthermore, the KIF13B motor domain translocated into the axon upon chemically induced linkage to the ound to dendritically localized cargo (Jenkins et al., 2012). Our data suggest that the KIF13B tail domain contributes to its polarized localization. The engineered kinesin toolbox provides an excellent platform to explore such hypotheses. 


\section{Future applications of the engineered motor proteins}

We believe that the toolbox of engineered kinesin motors could be widely used to investigate motor-cargo interactions and we foresee many different applications. The platform could be used to identify an interaction between cargo and any protein of interest. For example, coupling the vesicle-binding regions of the retrograde dynein motor or actin-based myosin motors will allow researchers to identify subunits that bind specific cargoes. Other interesting candidates to be used in the assay are adaptor proteins, which are known to play significant roles in the motor-cargo interaction (Akhmanova and Hammer, 2010; Fu and Holzbaur, 2014). Using adaptor proteins or truncated adaptor fragments in the platform will enable the identification of new adaptors and specific binding regions within an adaptor involved in motor-cargo interaction. Furthermore, the toolbox can be used to identify regulatory mechanisms that are involved in controlling motor-cargo binding, such as $\mathrm{Ca}^{2+}$ regulation or phosphorylation by specific kinases. For example, $\mathrm{Ca}^{2+}$ levels are proposed to mediate of KIF13B by cyc of KIF13B by cyclin-dependent kinase 5 mediates the KIF13B association with transien receptor potential vanilloid 1 (TRPV1) (Xing et al., 2012). Performing the assay in low o high $\mathrm{Ca}^{2+}$ environments or in the presence of kinase or phosphatase inhibitors could provide new insights into such regulatory mechanisms. A different application might be to use the assay to assess motor strength. It is known that one vesicle can bind multiple motors, which together regulate cargo transport (Gross et al., 2007). By using an additional motor domain, one could induce the generation of a double motor and assess cargo transport. Future studies should investigate motor preference in different cellular environments and stressors to identify regulatory mechanisms in cargo transport by cooperating motors.

\section{Limitations}

The engineered motor platform provides an efficient tool to study kinesin-cargo interactions. The assay depends on re-localization of cargo into distal tips and positive interactions between cargo and kinesin tail domain are efficiently detected. However, a disadvantage of the assay is the possibility to obtain false-negative results. These can arise when the expression level of either the motor or tail domain is low, resulting in only few motors capable of cargo transport. Furthermore, clear re-localization of cargo depends on a tight interaction with the kinesin tail and weaker or transient interactions might not be detected. To decrease the likelihood of obtaining false negatives, we use long-term rapalog treatment. This enables the motor and tail domain to interact with each other directly after protein synthesis and allows enough time for fully functional motors to re-localize cargo into axonal tips, thereby increasing the ability to pick up interactions even at lower expression levels or with weaker interactions. In addition, our quantitative approaches provide a method to pick up interactions even when only few motors are present or in case of weaker binding affinity.

In conclusion, we have developed a highly adaptable toolbox of engineered kinesin motors that allows for the selective transport of specific cargo with chemical induction to study kinesin-cargo interactions. We demonstrate that the assay is robust and has an easily interpretable readout, which enables identification of kinesin-cargo interactions with high specificity and has a wide-range of future applications, thereby providing an excellent toolbox to study kinesin-cargo interactions in living cells.

\section{AUTHOR CONTRIBUTIONS}

Conceptualization: J.J.A.H.; Methodology: J.J.A.H.; Validation: J.J.A.H.; Formal analysis: J.J.A.H.; Investigation: J.J.A.H.; Data curation: J.J.A.H.; Writing - original draft: J.J.A.H.; Writing - review \& editing: C.C.H.; Visualization: J.J.A.H.; Supervision: C.C.H.; Project administration: C.C.H.; Funding acquisition: C.C.H.

\section{MATERIALS AND METHODS}

Animal experiments were approved by the Dutch Animal Experiments Committee (DEC) and performed according to guidelines of Utrecht University, Dutch law (Wet op de Dierproeven, 1996) and European regulations (Guideline 86/609/EEC). Hippocampal neurons used in this study were obtained from embryonic day 18 (E18) stage embryos of both genders from female pregnant Wistar rats (Janvier) being at least 10 weeks old and were not involved in previous experiments. Rats were housed with a companion in transparent Plexiglas cages with wood-chip
bedding and paper tissue. They were kept in a $12 \mathrm{~h}$ light-dark cycle with a temperature of $22 \pm 1^{\circ} \mathrm{C}$ and provided with
unrestricted access to food and water.

Primary hippocampal neuron cultures and transfection

Primary hippocampal neuron cultures were prepared from E18 rat brains following protocols described previously (Kapitein et al., 2010a). Neurons were plated in a 12-well plate on coverslips coated with poly-L-lysine (37.5 $\mu \mathrm{g} /$ (NB) and $1 \%$ penicillin/streptomycin (Gibco) at $37^{\circ} \mathrm{C}$ and $5 \% \mathrm{CO}_{2}$.

Hippocampal neurons were transfected at day in vitro 7 (DIV7) using Lipofectamine 2000 (Invitrogen). DNA $(1.8 \mu \mathrm{g} /$ well $)$ was mixed with Lipofectamine $2000(3.3 \mu \mathrm{l} /$ well $)$ in $200 \mu \mathrm{l} \mathrm{NB}$ medium and incubated for 30 $\mathrm{min}$. The DNA/lipofectamine mixture was added to the neurons in transfection medium (NB medium supplemented with $0.5 \mathrm{mM}$ glutamine) and incubated for $45 \mathrm{~min}$ at $37^{\circ} \mathrm{C}$ and $5 \% \mathrm{CO}_{2}$. Neurons were then washed with NB medium and transferred to their original medium at $37^{\circ} \mathrm{C}$ and $5 \% \mathrm{CO}_{2}$ until fixation at DIV8. Rapalog (final concentration of $1 \mu \mathrm{M})$ was added directly after transfection

DNA and ShRNA constructs

The following DNA constructs used in this study were as described previously: $\beta \beta$-actin-HA- $\beta$-galactosidase (Hoogenraad et al., 2005), pGW2-TagBFP (Lipka et al., 2016), pGW2-NPY-GFP (Schlager et al., 2010), TfR-GF (Burack et al., 2000), GFP-LAMP1 (Farías et al., 2017), GFP-Rab3A (van Vlijmen et al., 2008), and Mito-GFP (Hoogenraad et al., 2003). PCR-based strategies were used to clone GW1-KIF5C_1-559-mRFP-(FKBP)2 using (KBa-Kif5 $C_{-}$- 559 -GFP (Addgene plasmid $\# 45059$ ) as a template and ligating it into a GW1-PEX-mRFP-(FKBP) 2 (NM 001294149.1) GW1-KIF1C 1-496-mRFP-(FKBP)2 2NM 145877.2), GW1-KIF13B 1-444-mRFP-(FKBP)2 (NM-015254.4), and GW1-KIF6 1 -500-mRFP-(FKBP)2 (XM-006244480.1). PCR-based strategies were used to create a GW1-FRB-3myc backbone from the GW1-HA expression vector and GW1-GFP-FRB (Kapitein et al, 2010c). Different KIFtd fragments (Table S1) were cloned into this backbone to generate GW1-FRB-3myc-KIFtd constructs. Truncated KIF16Btd fragments and mutated KIF16Btd were generated using PCR-based strategies with GW1-FRB-3myc-KIF16Btd as template and ligated into the GW1-FRB-3myc backbone. PCR-based strategies with GW1-FRB-3myc-KIF16Btd and GW1-FRB-3myc-KIF1Atd as templates were used to generate KIF1A_395-1559KIF16B_1101-1266 (KIF1A-PX) and KIF16B_395-1100-KIF1A_1560-1698 (KIF16B-PH), which were cloned into the GW1-FRB-3myc backbone.

Antibodies and reagents

The following antibodies and dilutions were used in this study for immunofluorescence experiments: mouse anti-Myc (1:200, Bio Connect), chicken anti- $\beta$-galactosidase (1:2500, Aveslab), mouse anti-Rab3A (1:200, BD Biosciences), goat anti-chicken-IgY conjugated to Alexa Fluor 405 (1:400, Abcam), goat anti-mouse-IgG conjugated to Alexa Fisher Scientific), Thermo Fisher Scientific), goat anti-mouse-lgG conjugated to Alexa Fluor 488 (1:400, Thermo imaging analysis NF-CF555 (Farias et al., 2016) was used. A reagent used in this study is rapalog (AP21967, TaKaRa).

\section{Immunofluorescence staining}

Transfected neurons were fixed at DIV8 with $4 \%$ formaldehyde and $4 \%$ sucrose in phosphate-buffered saline (PBS) at room temperature for $10 \mathrm{~min}$. Cells were then washed three times in PBS-CM (PBS, $1 \mathrm{mM} \mathrm{MgCl}, 0.1 \mathrm{mM}$ $\mathrm{CaCl}_{2}$ ), permeabilized with $0.2 \%$ Triton X-100 for $15 \mathrm{~min}$, and washed one time with PBS-CM, before incubation with $0.2 \%$ gelatin for $30 \mathrm{~min}$ at $37^{\circ} \mathrm{C}$. Next, neurons were incubated with primary antibodies diluted in $0.2 \%$ gelatin for $30 \mathrm{~min}$ at $37^{\circ} \mathrm{C}$, and washed three times in PBS-CM. This was followed by incubation with secondary antibody diluted in $0.2 \%$ gelatin for $30 \mathrm{~min}$ at $37^{\circ} \mathrm{C}$, and washing three times in PBS-CM. Finally, coverslips were mounted in Fluoromount (Invitrogen).

Fixed cells were imaged on: (1) a Nikon Eclipse 80i upright widefield fluorescence microscope, equipped with a Photometrics CoolSNAP HQ2 CCD camera and Nikon NIS Br software, using a Plan Fluor 40× N.A/1.30 oil objective; or (2) a Carl Zeiss LSM 700 confocal laser scanning microscope running ZEN2011 software, using
Plan-Apochromat $40 \times 11.30$ oil DIC objective. 


\section{Image analysis and quantification}

Classification of kinesin-cargo interaction. To determine which kinesins interact with a cargo, hippocampal neurons transfected with KIF5Cmd, FRB-KIFtd, and GFP-labeled cargo were visualized on a widefield fluorescence microscope. Coverslips were scanned for cells containing all three constructs. If the condition where rapalog was as kinesin-cargo interaction. If this was not the case, it was classified as no interaction between kinesin and cargo: Quantification of the percentage of cells with cargo transport. Once a kinesin-cargo interaction was identified, neurons expressing KIF5Cmd, FRB-KIFtd and GFP-labeled cargo were visualized on a widefield fluorescence microscope. Coverslips for both the condition without rapalog and the condition with addition of rapalog were scanned for cells containing all three constructs on a first-come-first-serve basis. Cells were classified as transporting if all three constructs colocalized in the distal tips of the cell and, from this, the percentage of cells showing cargo transport was calculated.

Quantification of the ratio of cargo intensity in growth cone to that in soma. For quantification of the ratio of cargo intensity in the growth cone to the intensity in the soma, confocal images of neurons transfected with KIF5Cmd, FRB-KIFtd and GFP-tagged cargo were acquired. The fluorescence intensity of the cargo was measure in five growth cones and the cell soma of a hippocampal neuron. This allowed calculation of the ratio of fluorescen intensity in a growth cone to that in the cell soma in the conditions without and with addition of rapalog. The ratio foun Live-cell imaging and analysis. Live-cell imaging experiments were performed on an inverted Niko Eclipse Ti-E confocal microscope equipped with a perfect focus system (Nikon), a CSU-X1-A1 Spinning Disc un (Yokogawa), a Photometrics Evolve 512 EMCCD camera (Roper Scientific) and a Plan Apo VC 100×N.A.1.40 oil objective. Coverslips were mounted in a Ludin chamber (life imaging services) and maintained in culture medium at $37^{\circ} \mathrm{C}$ and $5 \% \mathrm{CO}_{2}$ in a stage incubator (Tokai Hit) during image acquisition.

Growth cone imaging. For live-imaging of cargo accumulation in the growth cone, neurons expressing KIF5Cmd, KIF1Atd and GFP-NPY were visualized, and growth cones of different neurons were selected. Images of growth cones were acquired every $5 \mathrm{~min}$ for a total of $3 \mathrm{~h}$. After four acquisitions $(15 \mathrm{~min}, \mathrm{t}=0$ ) rapalog was added to a final concentration of $1 \mu \mathrm{M}$. Movies were processed using Fiji (Schindelin et al., 2012) and NPY intensity in the distal tip (determined from KIF5Cmd localization) at different time points was measured.

Imaging of axonal vesicle entry. Neurons expressing KIF5Cmd, KIF1Atd and GFP-NPY, were incubated for $30 \mathrm{~min}$ with NF-CF555, before imaging. Axons were identified from NF-CF555 staining, and movies in the of rapalog and $15 \mathrm{~min}$ after addition of rapalog $(1 \mu \mathrm{M}$ final concentration). Kymographs were generated using the Kymoreslicewide (GitHub: https://github.com/ekatrukha/KymoResliceWide) plugin for Fiji.

\section{REFERENCES}

Akhmanova, A., and Hammer, J.A. (2010). Linking molecular motors to membrane cargo. Curr. Opin. Cell Biol. Bentley, M., and Banker, G. (2016). The cellular mechanisms that maintain neuronal polarity. Nat. Rev. Neurosci.
17, 611-622.

Van Bergeijk, P., Adrian, M., Hoogenraad, C.C., and Kapitein, L.C. (2015). Optogenetic control of organelle
transport and positioning. Nature 518,111-114

Burack, M.A., Silverman, M.A., and Banker, G. (2000). The role of selective transport in neuronal protein sorting.

Encalada, S.E., and Goldstein, L.S.B. (2014). Biophysical challenges to axonal transport: Motor-cargo deficiencies
and neurodegeneration. Annu. Rev. Biophys, $43,141-169$

Farias, G.G., Britt, D.J., and Bonifacino, J.S. (2016). Imaging the polarized sorting of proteins from the golgi Farías, G.G., Cuitino, L., Guo, X., Ren, X., Jarnik, M., Mattera, R., and Bonifacino, J.S. (2012). Signal-mediated AP-1/Clathrin-dependent Sorting of Transmembrane Receptors to the Somatodendritic Domain of Hippocampa

Farías, G.G., Guardia, C.M., De Pace, R., Britt, D.J., and Bonifacino, J.S. (2017). BORC/kinesin-1 ensemble drives

arkhondeh, A., Niwa, S., Takei, Y., and Hirokawa, N. (2015). Characterizing KIF16B in neurons reveals a novel localization of early endosomes. J. Neurosci. 35, 5067-5086. Fu, M. meng, and Holzbaur, E.L.F. (2014). Integrated regulation of motor-driven organelle transport by scaffolding Gross, S.P., Vershinin, M., and Shubeita, G.T.T. (2007). Cargo transport: Two motors are sometimes better than one. Curr. Biol. $17,478-486$

Hirokawa, N., and Noda, Y. (2008). Intracellular transport and kinesin superfamily proteins, KIFs: Structure,

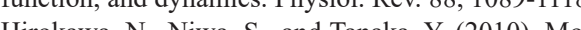
Hirokawa, N., Niwa, S., and Tanaka, Y. (2010). Molecular motors in neurons: Transport mechanisms and roles in
brain function, development, and disease. Neuron 68, 610-638.
Hoogenraad, C.C., Wulf, P., Schiefermeier, N., Stepanova, T., Galjart, N., Small, J.V., Grosveld, F., De Zeeuw,
C.I., and Akhmanova, A. (2003). Bicaudal D induces selective dynein-mediated microtubule minus end-directed C.I., and Akhmanova, A. (2003).
transport. EMBO J. 22, 6004-6015.

Hoogenraad, C.C., Milstein, A.D., Ethell, I.M., Henkemeyer, M., and Sheng, M. (2005). GRIP1 controls dendrite

Huang, C.F., and Banker, G. (2012). The translocation selectivity of the kinesins that mediate neuronal organelle transport. Traffic 13, 549-564.

Jacobson, C., Schnapp, B., and Banker, G.A. (2006). A change in the selective translocation of the kinesin-1 motor C.

Jenkins, B., Decker, H., Bentley, M., Luisi, J., and Banker, G. (2012). A novel split kinesin assay identifies motor Kapitein, L.C., Yau, K.W., and Hoogenraad, C.C. (2010a). Microtubule Dynamics in Dendritic Spines (Elsevier Inc.). Kapitein, L.C., Schlager, M.A., Kuijpers, M., Wulf, P.S., van Spronsen, M., MacKintosh, F.C., and Hoogenraad, Kapitein, L.C., Schlager, M.A., Van Der Zwan, W.A., Wulf, P.S., Keijzer, N., and Hoogenraad, C.C. (2010c). Kato, Y., Miyakawa, T., and Tanokura, M. (2018). Overview of the mechanism of cytoskeletal motors based on Kato, Y., Miyakawa, T., and Tanokura, M. (2018). Overview of the mechanism of cytoskeletal motors based on
structure. Biophys. Rev. 10, 571-581. Lawrence, C.J., Dawe, R.K., Christie, K.R., Cleveland, D.W., Dawson, S.C., Endow, S.A., Goldstein, L.S.B.,
Goodson, H.V.,Hirokawa, N., Howard, J., et al. (2004). A standardized kinesin nomenclature. J. Cell Biol. 167, 19-22. Lipka, J., Kapitein, L.C., Jaworski, J., and Hoogenraad, C.C. (2016). Microtubule-binding protein doublecortin-like kinase 1 (DCLK1) guides kinesin-3-mediated cargo transport to dendrites. Embo J. 1, 1-17.

Lo, K.Y., Kuzmin, A., Unger, S.M., Petersen, J.D., and Silverman, M.A. (2011). KIF1A is the primary anterograde motor protein required for the axonal transport of dense-core vesicles in cultured hippocampal neurons. Neurosci.

Maday, S., Twelvetrees, A.E., Moughamian, A.J., and Holzbaur, E.L.F. (2014). Axonal transport: cargo-specific
mechanisms of motility and tegulation. Neuron 84, 292-309.

Miki, H., Setou, M., Kaneshiro, K., and Hirokawa, N. (2001). All kinesin superfamily protein, KIF, genes in mouse Millecamps, S., and Julien, J.P. (2013). Axonal transport deficits and neurodegenerative diseases. Nat. Rev. Neurosci. $14,161-176$.

Nakata, T., and Hirokawa, N. (2003). Microtubules provide directional cues for polarized axonal transport through

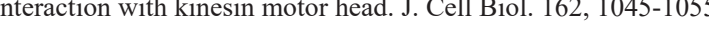

Nangaku, M., Sato-Yoshitake, R., Okada, Y., Noda, Y., Takemura, R., Yamazaki, H., and Hirokawa, N. (1994). KIF1B, Schindelin, J., Arganda-Carreras, I., Frise, E., Kaynig, V., Longair, M., Pietzsch, T., Preibisch, S., Rueden, C., Saalfeld,
S., Schmid, B., et al. (2012). Fiji: An open-source platform for biological-image analysis. Nat. Methods 9, $676-682$. Schlager, M.A., Kapitein, L.C., Grigoriev, I., Burzynski, G.M., Wulf, P.S., Keijzer, N., De Graaff, E., Fukuda, M.,

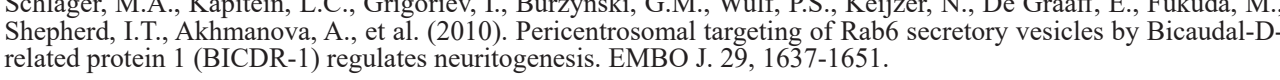

Steketee, M.B., Moysidis, S.N., Jin, X.L., Weinstein, J.E., Pita-Thomas, W., Raju, H.B., Iqbal, S., and Goldberg, J.L.
(2011). Nanoparticle-mediated signaling endosome localization regulates growth cone motility and neurite growth. NAS 108, 19042-19047.

Terada, S., and Hirokawa, N. (2000). Moving on to the cargo problem of microtubule-dependent motors in neurons.

van Vlijmen, T., Vleugel, M., Evers, M., Mohammed, S., Wulf, P.S., Heck, A.J.R., Hoogenraad, C.C., and van der Lett. 582, 2838-2842.

Wang, X., and Schwarz, T.L. (2009). The mechanism of $\mathrm{Ca}^{2+}$-dependent regulation of kinesin-mediated mitochondrial
motility. Cell 136, 163-174. Xing, B.M., Yang, Y.R., Du, J.X., Chen, H.J., Qi, C., Huang, Z.H., Zhang, Y., and Wang, Y. (2012). Cyclin-dependen kinase 5 controls TRPV1 membrane trafficking and the heat sensitivity of nociceptors through KIF13B. J. Neurosc

Yang, R., Bentley, M., Huang, C.-F., and Banker, G. (2016). Analyzing kinesin motor domain translocation in

Somi, R., Altelaar, M., and Hoogenraad, C.C. (2021). Combined knesin-1 and kinesin-3 activity drives axonal trafficking of TrkB receptors in Rab6 carriers. Dev. Cell 1-15. 


\section{SUPPLEMENTAL INFORMATION}

\section{Table S1. Overview of kinesin tail domains used in this study}

\begin{tabular}{|l|l|l|l|l|}
\hline Family & Kinesin & Tail domain & Accession number & Specie \\
\hline Kinesin-1 & KIF5A & $375-1032$ & NM_004984.4 & Human \\
\cline { 2 - 5 } & KIF5B & $374-963$ & NM_004521.3 & Human \\
\cline { 2 - 5 } & KIF5C & $376-955$ & NM_001107730.1 & Rat \\
\hline Kinesin-2 & KIF3A & $386-702$ & NM_001300792.2 & Human \\
\cline { 2 - 5 } & KIF17 & $376-1028$ & NM_001122819.3 & Human \\
\hline Kinesin-3 & KIF1A & $395-1698$ & NM_001294149.1 & Mouse \\
\cline { 2 - 5 } & KIF1B $\alpha$ & $390-1153$ & NM_001365953.1 & Human \\
\cline { 2 - 5 } & KIF1B $\beta$ & $390-1770$ & NM_015074.3 & Human \\
\cline { 2 - 5 } & KIF1C & $395-1103$ & NM_006612.6 & Human \\
\cline { 2 - 5 } & KIF13A & $397-1770$ & NM_001105566.3 & Human \\
\cline { 2 - 5 } & KIF13B & $443-1826$ & XM_006518620.4 & Mouse \\
\cline { 2 - 5 } & KIF16B & $395-1266$ & NM_001199865.2 & Human \\
\hline Kinesin-4 & KIF4A & $374-1129$ & IMAGE: 4538604 & Human \\
\cline { 2 - 5 } & KIF4B & $374-1234$ & NM_001099293.3 & Human \\
\cline { 2 - 5 } & KIF21A & $377-1661$ & NM_017641.4 & Human \\
\cline { 2 - 5 } & KIF21B & $410-1624$ & NM_017596.4 & Human \\
\hline
\end{tabular}
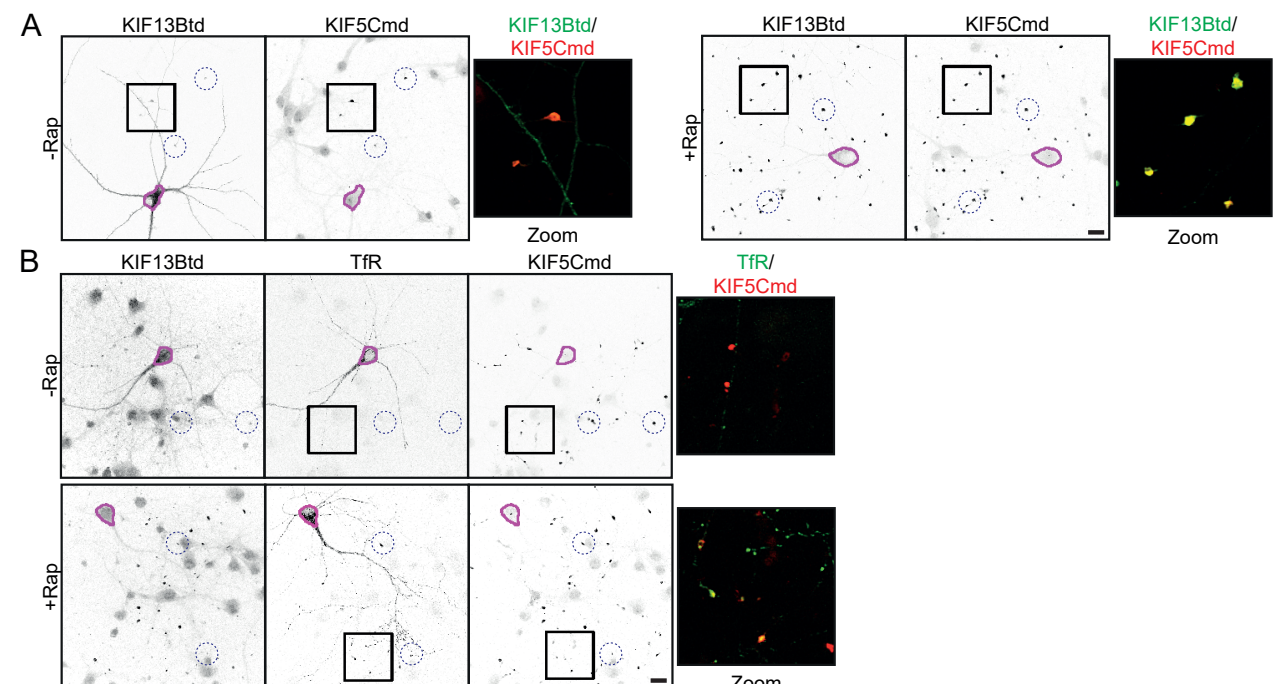

TiRM
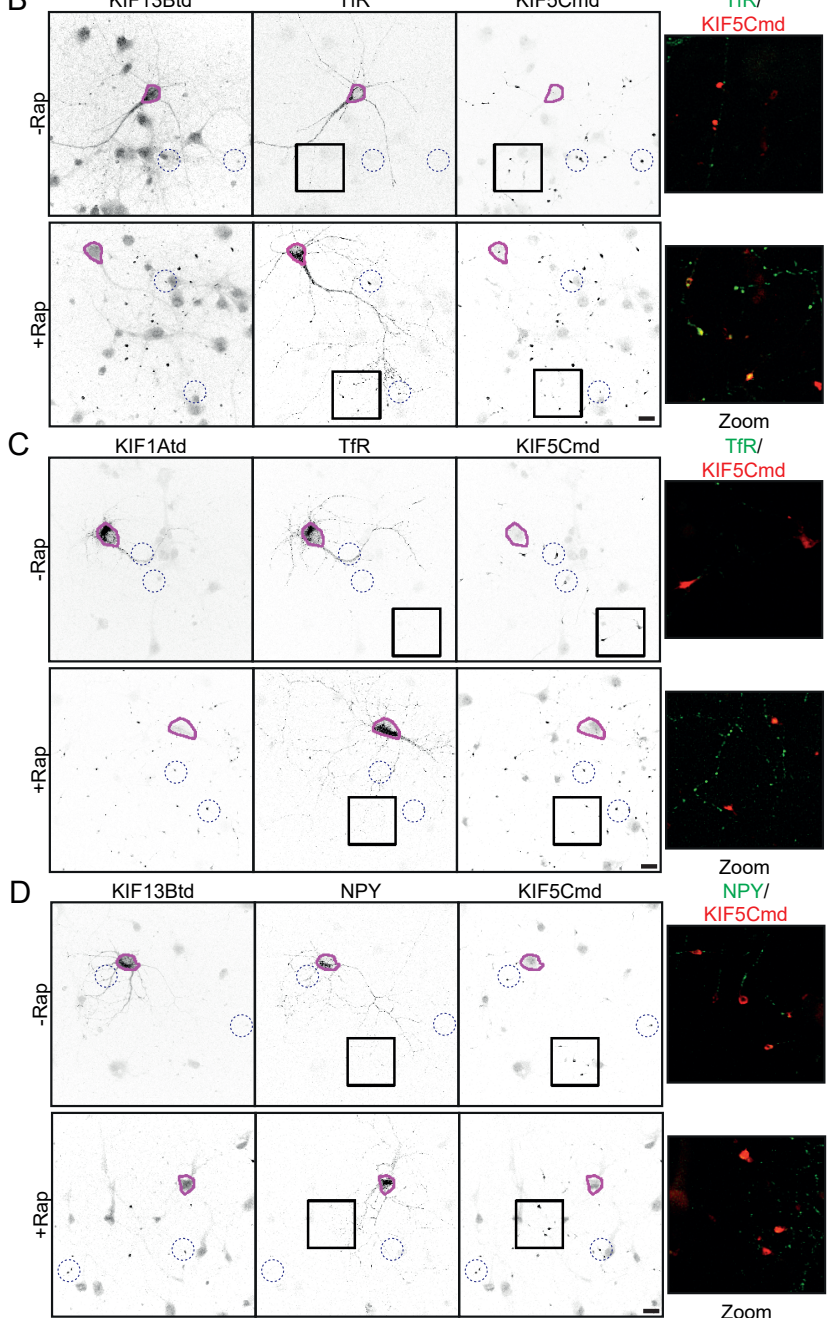

Figure S1. Negative interactions in the engineered kinesin assay

(A) Representative images of hippocampal neurons co-expressing FKBP-mRFP-KIF5Cmd and FRB-3mycIf $13 \mathrm{Btd}$ without (left) or with (right) addition of $1 \mu \mathrm{M}$ rapalog at transfection. The purple line shows the outline regions is shown. (B) Representative images of hippocampal neurons coexpressing FKBP-mRFP-KIF5Cmd, FRB3 myc-KIF13Btd and TfR-GFP without (top) or with (bottom) addition of $1 \mu \mathrm{M}$ rapalog at transfection. The purple line shows the outline of the cell soma and blue dotted circles indicate examples of axonal tips. A merged magnified view of the boxed regions is shown. (C,D) Representative images of hippocampal neurons co-expressing FKBPmRFP-KIF5Cmd, FRB-3myc-KIF1Atd and TfR-GFP(C) or FRB-3myc-KIF13Btd and NPY-GFP(D) without (top) or with (bottom) addition of $1 \mu \mathrm{M}$ rapalog at transfection. The puple line shows the oulline of the cell soma and Sue doted circles indicate examples of axonal tips. A merged magnified view of the boxed regions is shown 

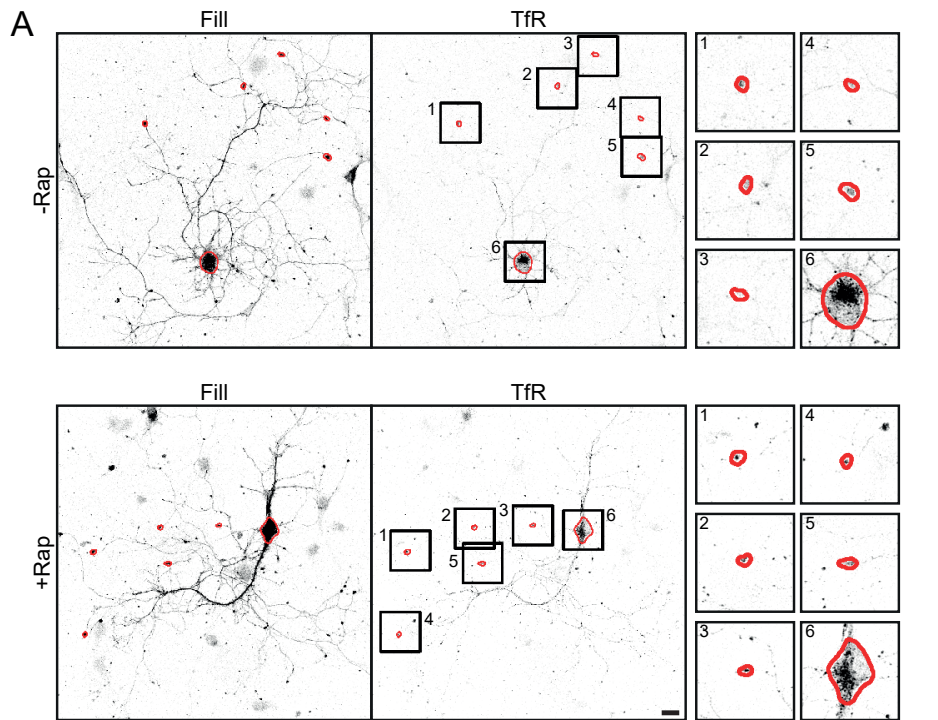

Figure S2. Quantification of negative kinesin-cargo interaction

(A) Representative example of the quantification method of the ratio of cargo intensity in distal tips over the som in neurons co-expressing FKBP-mRFP-KIF5Cmd, FRB-3myc-KIF1Atd and TfR-GFP. Distal tips and the soma are selected from the fill (HA- $\beta$-galoctidase) for both the conditions without (top) or with (bottom) rapalog (outlined in red). Cargo intensity in these regions of interest is then measured in the cargo channel. Magnified views of the regions of interst are shown in the small $(\mathrm{Bght}$ pants. $(\mathrm{B}, \mathrm{C})$ Quantifications of the ratio of cargo intensity in the

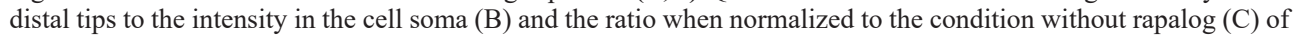
the example neurons depicted in (A). Results are mean \pm s.e.m. ( $\mathrm{n}=1$ cell). Scale bar: $20 \mu \mathrm{m}$.
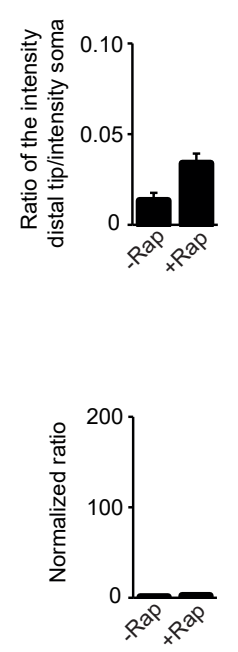
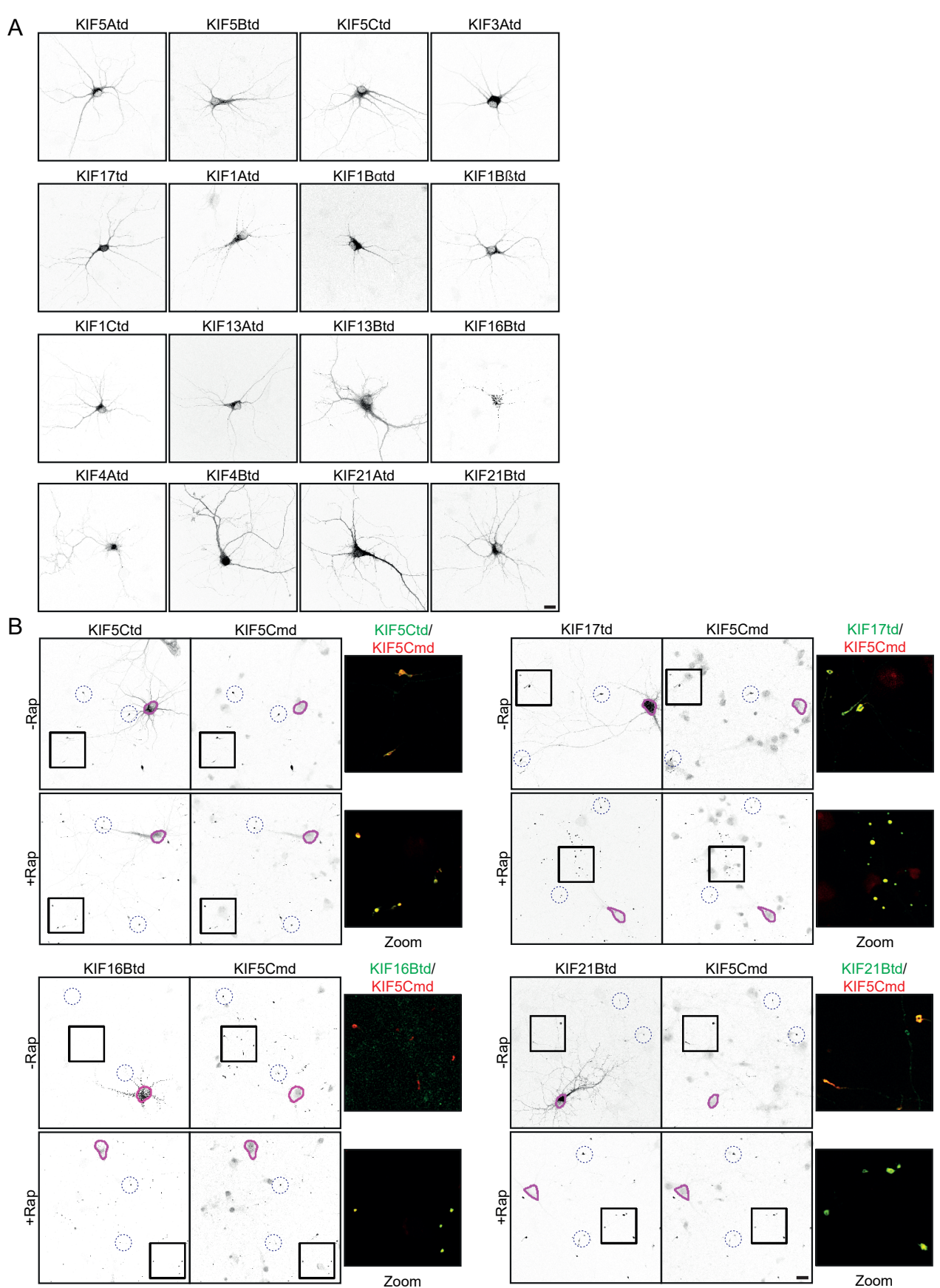

Figure S3. Localization of kinesin tail domains and validation of rapalog-induced binding

(A) Representative images of hippocampal neurons expressing FRB-3myc-KIFtd constructs. (B) Representative mages of hippocampal neurons co-expressing FKBP-mRFP-KIF5Cmd and FRB-3myc-KIF5Ctd, FRB-3mycKIF 17td, FRB-3myc-KIF16Btd, or FRB-3myc-KIF21Btd without (top) or with (bottom) addition of $1 \mu \mathrm{M}$ rapalog at transfection. The purple line shows the outline of the cell soma and blue dotted circles indicate examples of axonal tips. A merged magnified view of the boxed regions is shown. Scale bars: $20 \mu \mathrm{m}$ 


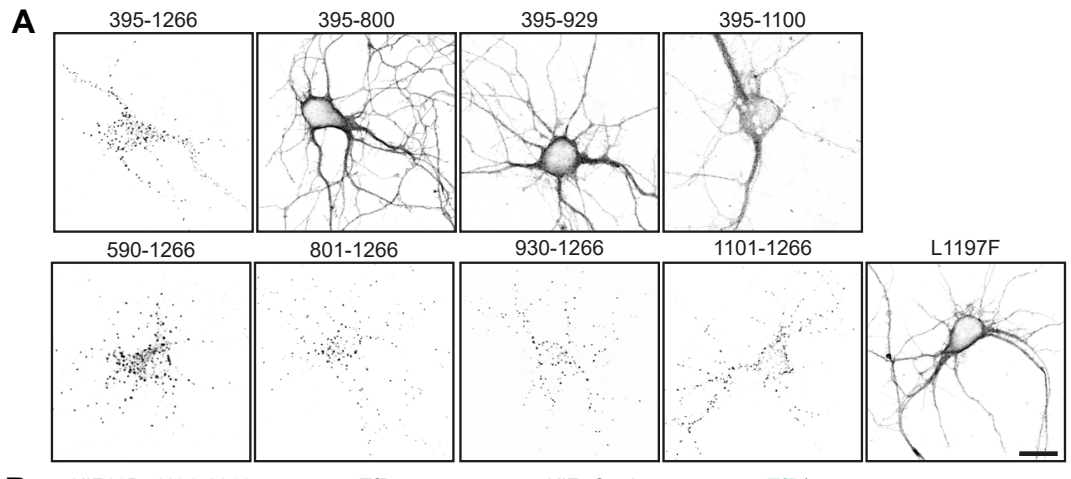

B
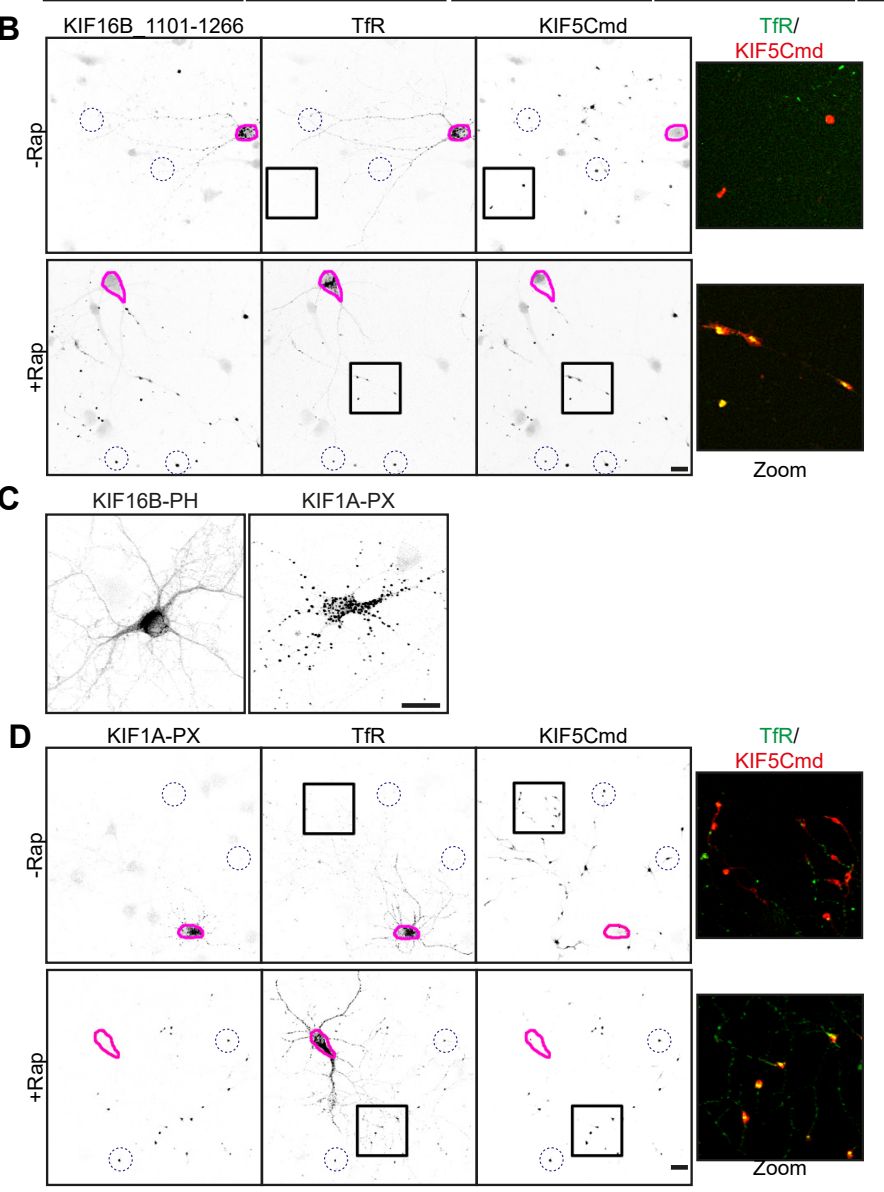

Figure S4. Localization of fragmented, mutated, and chimeric KIF16B constructs
(A) Representative images of hippocampal neurons expressing FRB-3myc-KIF16Btd fragments. (B) Representative images of hippocampal neurons co-expressing FKBP-mRFPKIF5Cmd, FRB-3myc-KIF16B 1101-1266 and TfRGFP without (top) or with (bottom) addition of $1 \mu \mathrm{M}$ rapalog at transfection. The purple line shows the outline of the cell soma and blue dotted circles indicate examples of axonal tips. A merged magnified view of the boxed regions is shown. (C) Representative images of hippocampal neurons expressing FRB-3myc-KIF16B-PH and FRBmyc-KIFIA-PX chimera constructs. (D) Representative images of hippocampal neurons coexpressing FKBPmRFP-KIF5Cmd, FRB-3myc-KIFIA-PX and TfR-GFP without (top) or with (bottom) addition of $1 \mu \mathrm{M}$ rapalog transfection. The purple line shows the outhne of he cell sona and blue dotted circles indicate examples of axona ips. A merged magnified view on
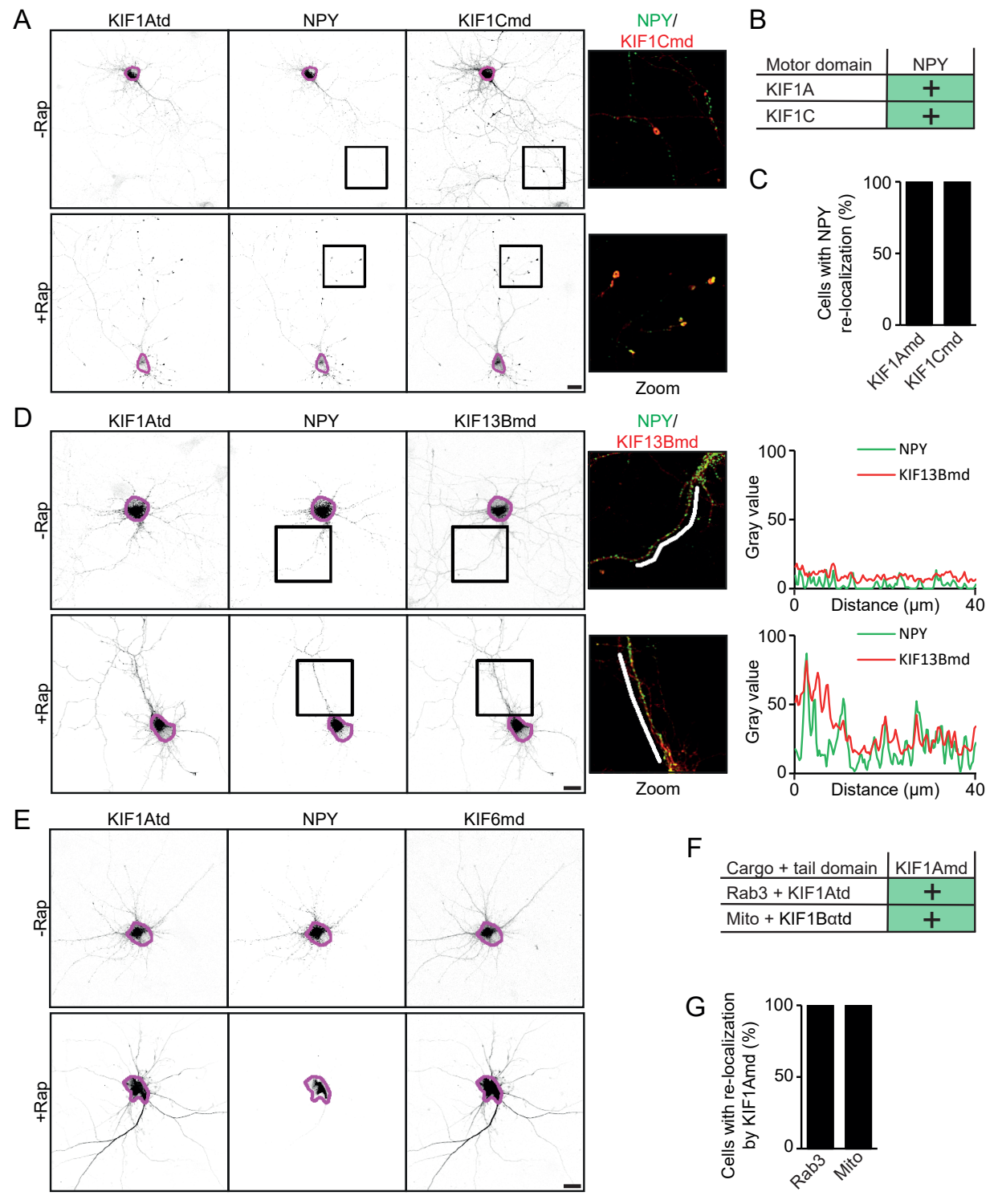

Cargo + tail domain $\mid$ KIF1Amd \begin{tabular}{l|c|} 
Rab3 + KIF1Atd & + \\
\hline Mito + KIF1Batd & + \\
\hline
\end{tabular}

$\mathrm{G} \cong$

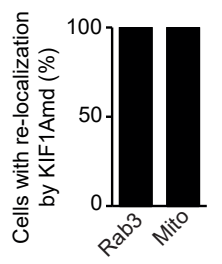

Figure S5. NPY re-localization by KIF13Bmd and KIF6md

(A) Representative images of hippocampal neurons co-expressing FKBP-mRFP-KIF1Cmd, FRB-3myc-KIF1Atd, and NPY-GF without (top) or with (bottom) addition of $1 \mu \mathrm{M}$ rapalog at transfection. The purple line shows the outline of the cell soma, and a merged magnified view of the boxed regions is shown. (B) Overview of the KIF motor domain constructs a dhir abity to transport DCV motor domains. Results are mean $\pm \mathrm{s}$. $\mathrm{m}$. ( $\mathrm{N}=2$ independent experiments, $\mathrm{n}=60$ cells). (D) Representative im $\mathrm{K}$ hippocampl neurons co-expressing FKBP-mRFP-KIF13Bmd, FRB-3myc-KIF1Atd, and NPY-GFP withous (top) or with (bottom) addition of $1 \mu \mathrm{M}$ rapalog at transfection. A merged magnified view of the boxed regions is shown. Graphs show the NPY (green) and KIF13Bmd (red) intensity along the line marked in the zooms. (E) Representative images of hippocampal neurons coexpressing FKBP-mRFP-KIF6md, FRB-3myc-KIF1Atd, and NPY-GFP without (top) or with (bottom) addition of $1 \mu \mathrm{M}$ rapalog at transfection. (F) Overview of axonal cargo for which dendritic re-localization is observed by KIF IAmd in the engineered kinesin assay screen. (G) Quantification of the percentage of cells in which cargo vesicles are re-localized into dendrites in the assay by KIF1Amd. Results are meants.e.m. $(\mathrm{N}=2$ independent experiments, $\mathrm{n}=60$ cells). Scale bars: $20 \mu \mathrm{m}$. 


\section{Specific KIF1A-adaptor interactions control selective cargo recognition}

Jessica J. A. Hummel ${ }^{1}$, Casper C. Hoogenraad ${ }^{1,2}$

J Cell Biol (2021);220:e202105011

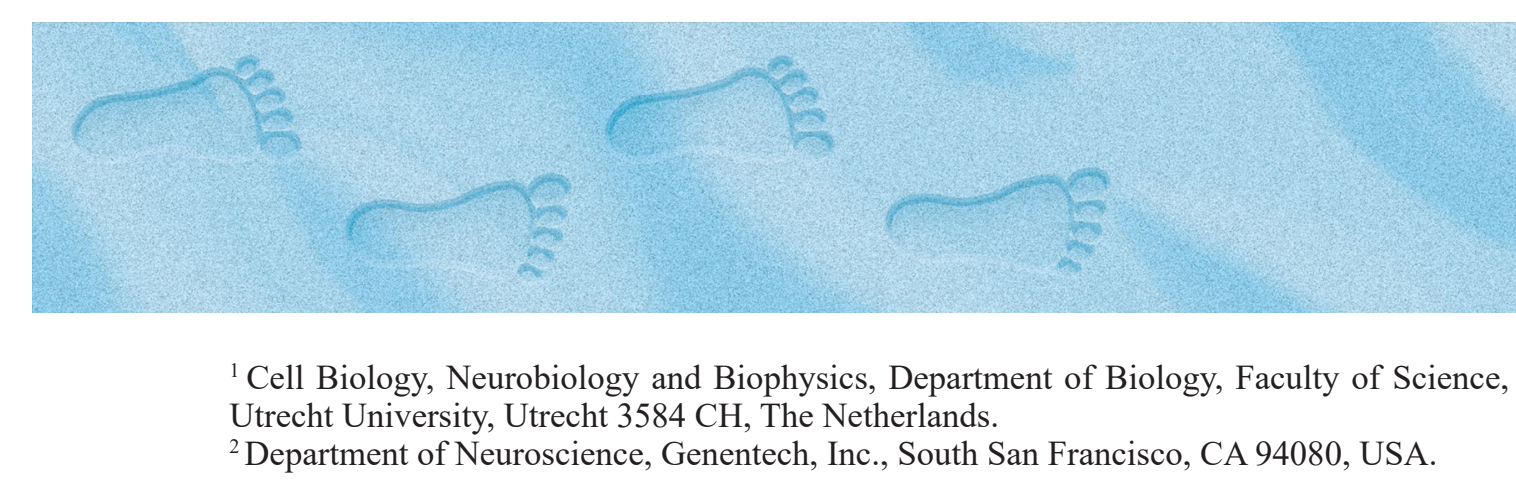




\section{ABSTRACT}

Intracellular transport in neurons is driven by molecular motors that carry many different cargos along cytoskeletal tracks in axons and dendrites. Identifying how motors interact with specific types of transport vesicles has been challenging. Here, we use engineered motors and cargo adaptors to systematically investigate the selectivity and regulation of kinesin-3 family member KIF1A-driven transport of dense core vesicles (DCVs), lysosomes, and synaptic vesicles (SVs). We dissect the role of KIF1A domains in motor activity and show that CC regulates autoinhibition, $\mathrm{CC} 2$ regulates motor dimerization, and $\mathrm{CC} 3$ and $\mathrm{PH}$ mediate cargo binding. Furthermore, we identify that phosphorylation of KIF1A is critical for binding to vesicles. Cargo specificity is achieved by specific KIF1A adaptors; MADD/Rab3GEP links KIF1A to SVs, and Arf-like GTPase Arl8A mediates interactions with DCVs and lysosomes. We propose a model where motor dimerization, posttranslational modifications, and specific adaptors regulate selective KIF1A cargo trafficking.

\section{INTRODUCTION}

Molecular motors use the cellular cytoskeleton to transport cargos throughout the cell. Transport of cargos and other building blocks is crucial for many cellular functions and is especially important in highly complex cells such as neurons. In neurons, cargos (such as protein complexes, membranous vesicles, organelles, and mRNA) are often generated in the cell body and transported over long distances into the correct compartments (Bentley and Banker, 2016). Kinesin, dynein, and myosin are molecular motors that transport these cargo into the axon and dendrites. Tight regulation of the transport machinery is critically important to ensure that cargo is picked up from and delivered to the right place at the right time (Hirokawa et al., 2010; van den Berg and Hoogenraad, 2012). Disruption of the transport machinery leads to neuronal disfunction, and genetic mutations in motor proteins have been linked to various neurological diseases in humans (Millecamps and Julien, 2013; Franker and Hoogenraad, 2013). For example, mutations in the molecular motor KIF1A, a kinesin-3 family member, have been reported to be involved in hereditary spastic paraplegias (Gabrych et al., 2019). KIF1A is a dimeric motor that drives transport to both axons and dendrites. In neurons, KIF1 A exists in an inactive, autoinhibited state, in which the C-terminal tail domain folds back on the N-terminal motor domain (Hammond et al., 2009). Autoinhibition is a common regulation mechanism for kinesin motor proteins (Verhey and Hammond, 2009). Several mechanisms have been found to release autoinhibition, among which are binding of cargo vesicles (Coy et al., 1999; Soppina et al., 2014) or binding to adaptor proteins (Siddiqui et al., 2019; Fu and Holzbaur, 2013). KIF1A and UNC104, its orthologue in Caenorhabditis elegans and Drosophila, were originally identified as the primary motors for axonal transport of synaptic vesicles (SVs; Hall and Hedgecock, 1991; Okada et al., 1995). This was supported

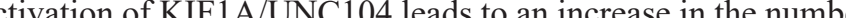
S SVs at the synapse, whereas d

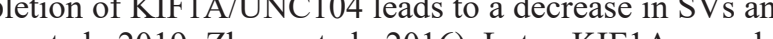
(Chiba et al., 2019; Zhang et al., 2016). Later, KIF1A was als found to be the primary anterograde motor for transport of dense core vesicles (DCVs; Zahn et al., 2004; Lo et al., 2011). Unlike SVs, DCVs are transported from the Golgi apparatu to the plasma membrane in both axons and dendrites (de Wit et al., 2006). Furthermore, in nonneuronal cells, KIF1A has been implicated in transport of organelles, such as lysosomes (Guardia et al., 2016). While KIF1A drives trafficking of several selective vesicle types, it remains unclear how KIF1A specificity is achieved for each cargo.

The interaction between motor protein and cargo is often mediated by adaptor proteins (Hirokawa et al., 2010). Different adaptor proteins have been proposed to mediate the KIF1A-cargo interaction. For example, liprin- $\alpha$ has long been suggested as an adaptor protein for KIF1A-mediated SV transport (Shin et al., 2003; Miller et al., 2005). In addition, tetratricopeptide repeat, ankyrin repeat and coiled-coil containing 2 (TANC2) was found to be a KIF1A interacting partner. However, it was recently shown that liprin- $\alpha$ and TANC2 might not function as classic adaptors, but rather as signposts recruiting KIF1A-driven cargo to dendritic synapses (Stucchi et al., 2018). The Arf-like small G protein, Arl8, has also been implicated in transport of SVs, and its loss of function decreases axonal trafficking of SVs and accumulates the cargo in the proximal axon (Klassen et al, 2010). GTP-Arl8 bound to SVs releases UNC-104 autoinhibition, thereby promoting cargo transport (Niwa et al., 2016). Mammalian Arl8 has two isoforms, Arl8A and Arl8B, which in nonneuronal cells localize on lysosomes (Hofmann and Munro, 2006). Furthermore, Arl8B was found to mediate lysosomal transport by kinesin-1 family motors in both nonneuronal and neuronal cells (Rosa-Ferreira and Munro, 2011; Farías et al., 2017).

Differentially expressed in normal and neoplastic cells/MAP kinase activating death domain (DENN/MADD or Rab3-GEP, further referenced as MADD) is a protein that has multiple roles, for example in neurotransmission (Miyoshi and Takai, 2004) and SV trafficking (Tanaka et al., 2001). It was also found to be an essential factor in transport of Rab3-containing SVs by KIF1B $\beta$ and KIF1A (Niwa et al, 2008). Thus, several KIF1A adaptor proteins are candidates for selective cargo bin how KIF1A-adaptor interactions control selective cargo recognition is currently lacking. To better understand how KIF1A interacts with different types of cargos, we used engineered motors to identify the mechanism of the KIF1A interaction with DCVs, lysosomes, and SVs. Using an inducible assay, we mapped the region in the KIF1A tail domain that is involved in the association with different cargos and found that the KIF1APH and CC3 domain are required for interaction with all three vesicle types. These findings were further validated using KIF1A depletion and rescue experiments. We also show that KIF1A motor activity is regulated by autoinhibition via $\mathrm{CC} 1$ as well as motor dimerization via $\mathrm{CC} 2$. In addition, we ind that KIF1A-cargo binding depends on phosphorylation events by calmodulin-dependent protein kinase II (CaMKII). Finally, we show that Arl8A acts as an adaptor for KIF1A mediating the interaction with DCVs and lysosomes, whereas MADD is an adaptor for SVs, suggesting a model in which KIF1A-cargo selectivity is achieved by specific adaptors

\section{RESULTS}

\section{Engineering motors to identify cargo for KIF1A in neurons}

Engineered motors can be used to identify kinesin-cargo interactions. Here, we used the motor domain of the kinesin-1 family member KIF5C (KIF5Cmd) fused to a FKBP domain and the tail domain of KIF1A (KIF1 Atd) to a FKBP12-rapamycin-binding (FRB) domain (Fig 1A,B). Addition of the chemical dimerizer AP21968, a rapamycin analogue (rapalog), leads to linkage of the FKBP and FRB domain (Hoogenraad et al., 2003; Kapitein et al., 2010a), thereby generating a full kinesin protein with motor and cargo binding functionalities. The constitutively active KIF5Cmd traffics selectively into the axon (Jacobson et al., 2006), which provides a clear readout of cargo relocalization into axonal tips after addition of rapalog in case of an interaction between KIF1Atd and cargo (Fig 1A). Validation of the assay was done by coexpressing FKBP-mRFP-KIF5Cmd and FRB-3myc-KIF1Atd constructs in hippo by immunostaining with myc antibodies. Addition of rapalog leads to a clear relocalization of KIF1Atd into axonal tips, where it colocalizes with KIF5Cmd (Fig SIA). This was quantified by the ratio of KIF1Atd intensity in a distal tip over KIF1Atd intensity in the soma (Fig 1C). Next, we used these engineered motors to screen for an interaction of different vesicl populations with KIF1A. Previously, DCVs, lysosomes and SVs have been described as cargo for KIF1A (Lo et al., 2011; Guardia et al., 2016; Hall and Hedgecock, 1991). For each vesicle type a GFP-tagged marker protein was used to label the vesicle population: neuropeptide $Y$ NPY) forDCVs (Kwinteretal , 2009), lysosomal-associated membrane protein 1(LAMP1) for lysosomes (Chen etal., 1985), and Rab3 for SVs (Fischer von Mollard et al., 1990). To identify an assocition between these vesicles and KIF1A hippocol with FKBP-mRFP-KIF5Cmd, FRB-3myc-KIF1Atd, and GFP-tagged marker proteins. 


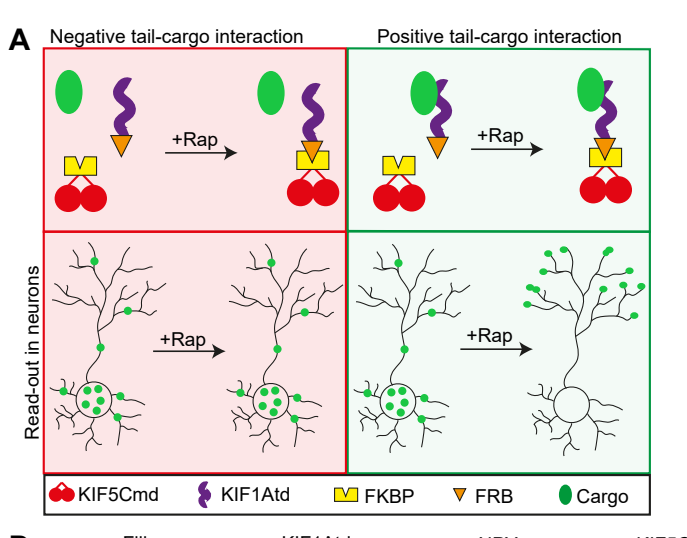

B

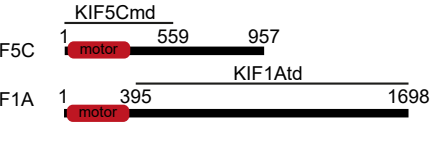

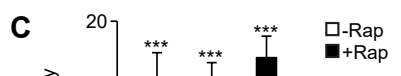
illi.
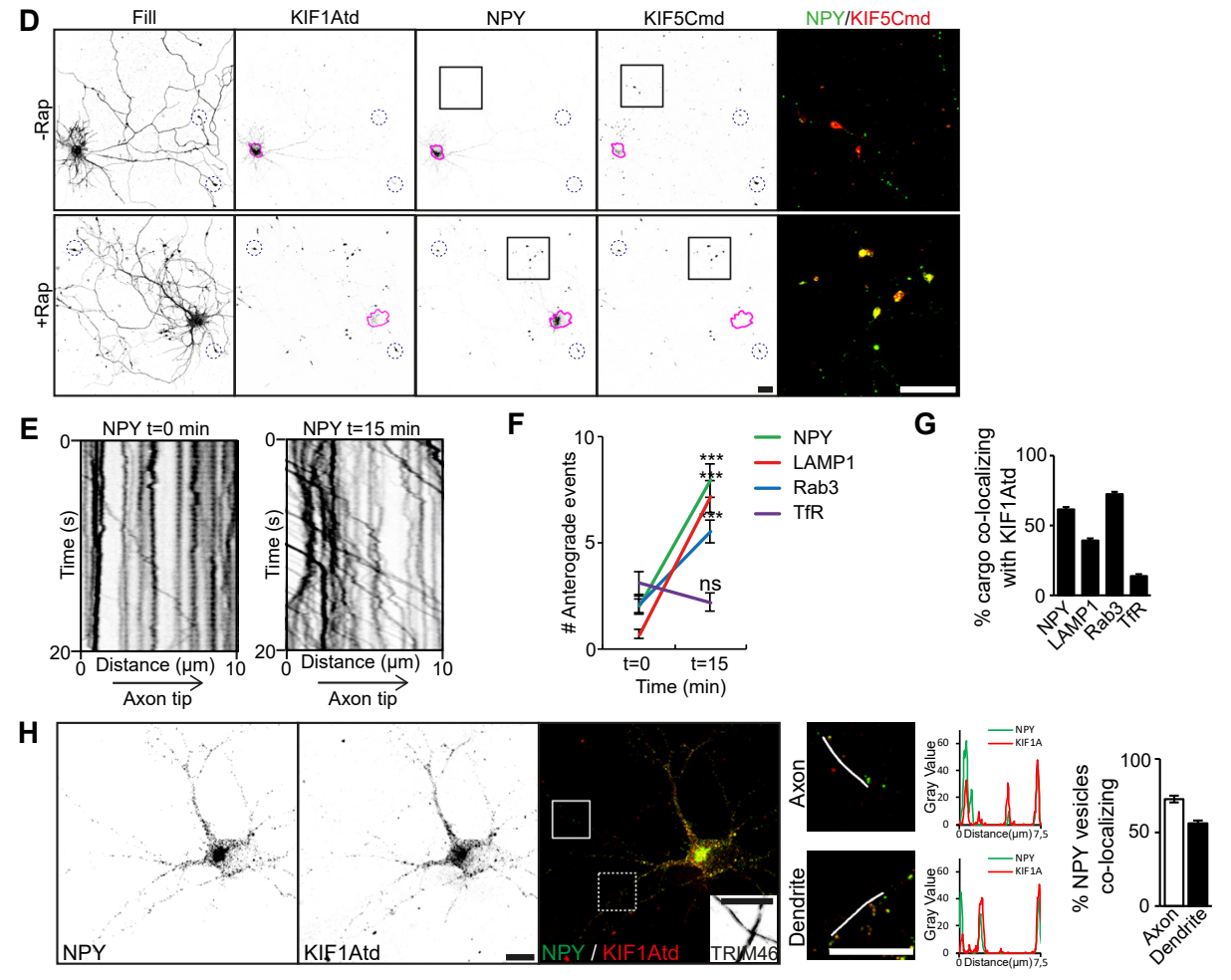

igure 1. Identification of KIF1 $\mathrm{A}$ cargo in neurons

Figure 1. Identification of KIF1A cargo in neurons
Identification of KIF1A cargos using engineered motors and evaluation of KIF1A-cargo interaction in neuronal compartments. (A) Schematic depiction of the engineered motor assay in case of a negative (left) or positive in a neuron. (B) Schematic depiction of the structure of KIF5C and KIF1A motors. (C) Quantification of the normalized ratio of cargo intensity in axonal tips to that of the soma of hippocampal neurons coexpressing FRB-3myc-KIF1Atd, cargo-GFP, and FKBP-mRFP-KIF5Cmd without rapalog or with addition of $1 \mu \mathrm{M}$ rapalog $(\mathrm{N}=1, \mathrm{n}=15)$. (D) Representative images of hippocampal neurons coexpressing FRB-3myc-KIF1Atd, NPY-GFP, and FKBP-mRFP-KIF5Cmd without (upper panels) or with (lower panels) addition of $1 \mu \mathrm{M}$ rapalog. Pink lines mark the cell soma. Blue dotted circles indicate examples of axonal tips. Zooms of the boxed regions are shown as a merge on the right. (E) Representative kymographs showing movement of NPY vesicles in the AIS before (left) and $15 \mathrm{~min}$ after (right) addition of $1 \mu \mathrm{M}$ rapalog. (F) Quantification of the axonal entries of cargo befor and $15 \mathrm{~min}$ after addition of $1 \mu \mathrm{M}$ rapalog in neurons expressing FRB-3myc-KIF1Atd, cargo-GFP, and FKBPKIFP-KIF
White boxes on the merged image mark the axon (continuous line) and a dendrite (dotted line). TRIM46 staining in the axon is shown in the merged image. Merged zooms of the boxes are depicted on the right. Graphs show the NPY (green) and KIFA (red) intente Deta a displaye as neentSEM. Man-Whincy U

Addition of rapalog led to relocalization of all three cargos into axonal tips (Fig 1D; Fig S1B,D). Relocalization was not observed for endosomal vesicles present in the somatodendritic region, which were labeled using GFP-tagged transferrin receptor (TfR Fig S1F; Burack et al., 2000), indicating that these vesicles do not interact with KIF1A. Relocalization of cargos was clear from quantifications, which show that the ratio of marker intensity in the axonal tip to that of the soma increases $\sim 15$-fold for DCVs and lysosomes and $\sim 10$-fold for SVs (Fig 1C). We further verified these interactions by live-cell imaging. For this, we imaged the axon initial segment (AIS), where in case of an interaction between of rapalog. Kymographs show that already $15 \mathrm{~min}$ after rapalog addition, axonal vesicle entry increased for DCVs (Fig 1E), lysosomes (Fig S1C), and SVs (Fig S1E), but not dendritic vesicles (Fig S1G), which was confirmed by quantifications (Fig 1F). Together, these data show that in hippocampal neurons, KIF1A interacts with DCVs, lysosomes, and SVs, but not with TfR-positive endosomal vesicles.

Association of KIF1A with different cargo is not restricted to neuronal compartments

KIF1A interacts with three distinct vesicle populations in hippocampal neurons, raisin the question of how specificity for a cargo is achieved. One possibility is that KIF1A associates with different vesicle populations in different neuronal compartments. First, we investigated the colocalization of our marker proteins on vesicles. We found that there was almost no colocalization between LAMP1-containing lysosomes and either NPY or Rab3 vesicles. However, we did observe that $50 \%$ of the Rab3 vesicles also contained PYY in both axonal and dendritic compartments, suggesting that there is some overlap between these vesicle types (Fig S2A). Next, we looked at colocalization of KIF1Atd with different vesicle types in specific neuronal compartments. We found that KIF1Atd colocalizes with DCVs, lysosomes and SVs in both the axon and dendrites (Fig 1G,H; Fig S2B). Further analysis revealed that DCVs and SVs show a slightly higher colocalization in the axon, whereas lysosomes colocalize slightly more with KIFlAtd in dendrites (Fig $1 \mathrm{H}$; Fig S2B). However, there is no indication of any preference for KIF1A colocalization with a specific cargo in a specific compartment. Low colocalization was observed for S2B). These resuls demonstrate that KIF1A specificity for a cargo is not restricted to axons or dendrites.

\section{KIF1A interacts with vesicles via its $\mathrm{PH}$ and $\mathrm{CC} 3$ domains}

As specificity of KIF1A for a cargo is not achieved by localization in different neuronal compartments, we wondered whether different vesicles interact with different domains of the KIF1A motor as a mechanism to achieve selectivity. The KIF1A tail domain consists of three coiled-coil (CC) domains with a forkhead-associated (FHA) domain between $\mathrm{CC} 1$ and $\mathrm{CC} 2$ at the $\mathrm{N}$-terminal side and a PH domain at the C-terminal side (Fig 2A). We generated severa fragments that lack the N-terminus of KIF1 Atd (Fig 2A). These were expressed in hippocampal neurons to visualize their localization pattern and subjected to our engineered motor assay to identify cargo interactions. Expression of KIF1A 657-1698 and KIF1A_1106-1698 in hippocampal neurons showed a punctate more axonal localization comparable to KIF1Atd, whereas KIF1A 1560-1698 showed a diffuse localization that was more dendritic (Fig 2B,C; Fig S3A). This suggests that KIF1A 657-1698 and KIF1A 1106-1698 associate with vesicles, but the $\mathrm{PH}$ domain alone is not sufficient for cargo interaction. 
Each fragment was then screened in our motor assay for an interaction with DCVs, lysosomes, and SVs. We used the following baseline criterion to identify positive cargo interactions: the ratio of cargo in distal tips over the soma is $>5 \%$ of the KIF1 Atd signal. This showed that KIF1A 657-1698, lacking only the $\mathrm{CC} 1$ and FHA domain, was able to bin all three cargos. The ratio of NPY intensity in the axonal tips to that of the soma increased for KIF1A 657-1698 compared with KIF1Atd, whereas for LAMP1 and Rab3, the ratio decreased for KIF1A 657-1698 with respect to KIF1Atd (Fig 2D). KIF1A 1106-1698, having a punctate localization pattern, was found to interact with DCVs, lysosomes, and SVs, although the association with each cargo was decreased strongly when compared with KIF1Atd (Fig 2D). The shortest fragment, KIF1A_1560-1698, containing only the PH domain, was unable to bind any of the cargo (Fig 2D). This was expected from its diffuse distribution and confirms that the $\mathrm{PH}$ domain alone is not sufficient for cargo interaction.

To further identify the minimum binding domain of the KIF1A tail that interacts with cargo, we then examined truncations of KIF1Atd lacking the PH domain. KIF1Atd was truncated from the $\mathrm{C}$-terminus or from both the $\mathrm{N}$ - and $\mathrm{C}$-termini to generate five KIF1A fragments: KIF1A 395-752, KIF1A 395-1105, KIF1A-395-1559, KIF1A 657-1559, and KIF1A 1106-1559 (Fig 2E). The distribution pattern of these constructs was diffuse, with the latter three constructs having a more dendritic localization compared with KIF1Atd (Fig 2F,G; Fig S3A). The screen from our motor assay shows that none of these construct can associate with DCVs or lysosomes. For SVs, positive interactions were detected with KIF1A 395-1105 and KIF1A_657-1559 (Fig 2H), suggesting that two sites might be involved in the interaction, one site in KIF1A 395-1105 and one site in KIF1A 1106-1698. Lastly, two constructs lacking either $\mathrm{CC} 2$ or $\mathrm{CC} 3$ (td $\triangle \mathrm{CC} 2$ and td $\triangle \mathrm{CC} 3$; Fig 2I) were generated. Whereas KIF1Atd $\triangle C C 2$ showed a similar distribution pattern as KIF1Atd, KIF1Atd $\triangle \mathrm{CC} 3$ exhibited a diffuse somatodendritic localization (Fig 2J,K; Fig S3A). The engineere motor assay shows that KIF1Atd $\triangle \mathrm{CC} 2$ associates with all three vesicle populations, where an interaction comparable to KIF1Atd was observed for DCVs and SVs and a slightly weaker binding for lysosomes. In contrast, KIF1Atd_ $\triangle \mathrm{CC} 3$ could not interact with any of the cargos (Fig 2L). In summary, these results suggest that both the $\mathrm{PH}$ and $\mathrm{CC} 3$ domain of KIF1Atd are necessary for interaction with DCVs, lysosomes, and SVs (Fig 2M,N)

\section{The KIF1A PH and CC3 domains are required for cargo trafficking}

To verify the importance of the $\mathrm{PH}$ and $\mathrm{CC} 3$ domains of KIF1A for cargo interaction, we performed KIF1A knockdown (KD) and rescue experiments. First, we generated KIF1A deletion constructs lacking the $\mathrm{PH}, \mathrm{CC} 1, \mathrm{CC} 2$, or CC3 domain (KIF1A_ $\Delta \mathrm{PH}, \mathrm{KIF} 1 \mathrm{~A} \_\Delta \mathrm{CC} 1$, KIF1A_ $\Delta$ CC2, and KIF1A_ $\triangle \mathrm{CC} 3$; Fig 3A).Comparison of the localization and polarity indices of these deletion mutants to full-length KIF1A (KIF1A FL), showed that KIF1A $\triangle \mathrm{CC} 1$ and KIF1A $\triangle \mathrm{CC} 2$ have a similar vesicular-like pattern, whereas KIF1A $\Delta \mathrm{PH}$, and KIF $1 \mathrm{~A} \_\Delta \mathrm{CC} 3$ have a diffuse unpolarized pattern (Fig 3B,C; Fig S4A), suggesting that the latter two are not associated with vesicles. KD of KIF1A in hippocampal neurons gave different phenotypes for each vesicle population. Depletion of KIF1A resulted in decreased axonal motility of DCVs (Fig 3D). Quantifications show that there is an increase in static traces and a decrease in both anterograde and retrograde traces (Fig 3E). The decreased vesicle motility phenotype was rescued by KIF1A_FL and KIF1A_ $\Delta C C 1$, but not by KIF1A_ $\Delta$ PH, KIF1A_ $\Delta C C 2$, or KIF1A $\triangle$ CC3 (Fig 3E). Lysosomes showed a similar decreased axonal motility phenotype upon KIF1A depletion (Fig 3F,G). This phenotype was rescued by KIF1A FL, KIFIA $\Delta \mathrm{CC} 1$, and KIF1A $\Delta \mathrm{CC} 2$, but not by KIF1A $\Delta \mathrm{PH}$ or KIF1A $\Delta \mathrm{CC} 3$ (Fig 3G). For SVs, we found two phenotypes upon depletion of KIF1A. SVs, visualized by overexpression of marke 3ab3, accumute in hecels soma in KIF1AKD (Fig 3H, This was rescoed by KIF1A FL, KIF1A $\triangle \mathrm{CC1}$, and KIF1A $\triangle \mathrm{CC} 2$, but KIF1A_ $\Delta C C 1$, and KIFlA_ $\Delta C C 2$, but not by KIF1A__PH or KIF1A_ $\Delta$ CC3 (Fig 3H,J). Accordingly, endogenous Rab3 vesicles were less transported along the axon upon KIF1A depletion (Fig 3I). The ratio of Rab3 intensity in the distal over the proximal axon indicates decrease in SVs distributed in the distal axon in KIF1A KD compared with control (Fig 3K)

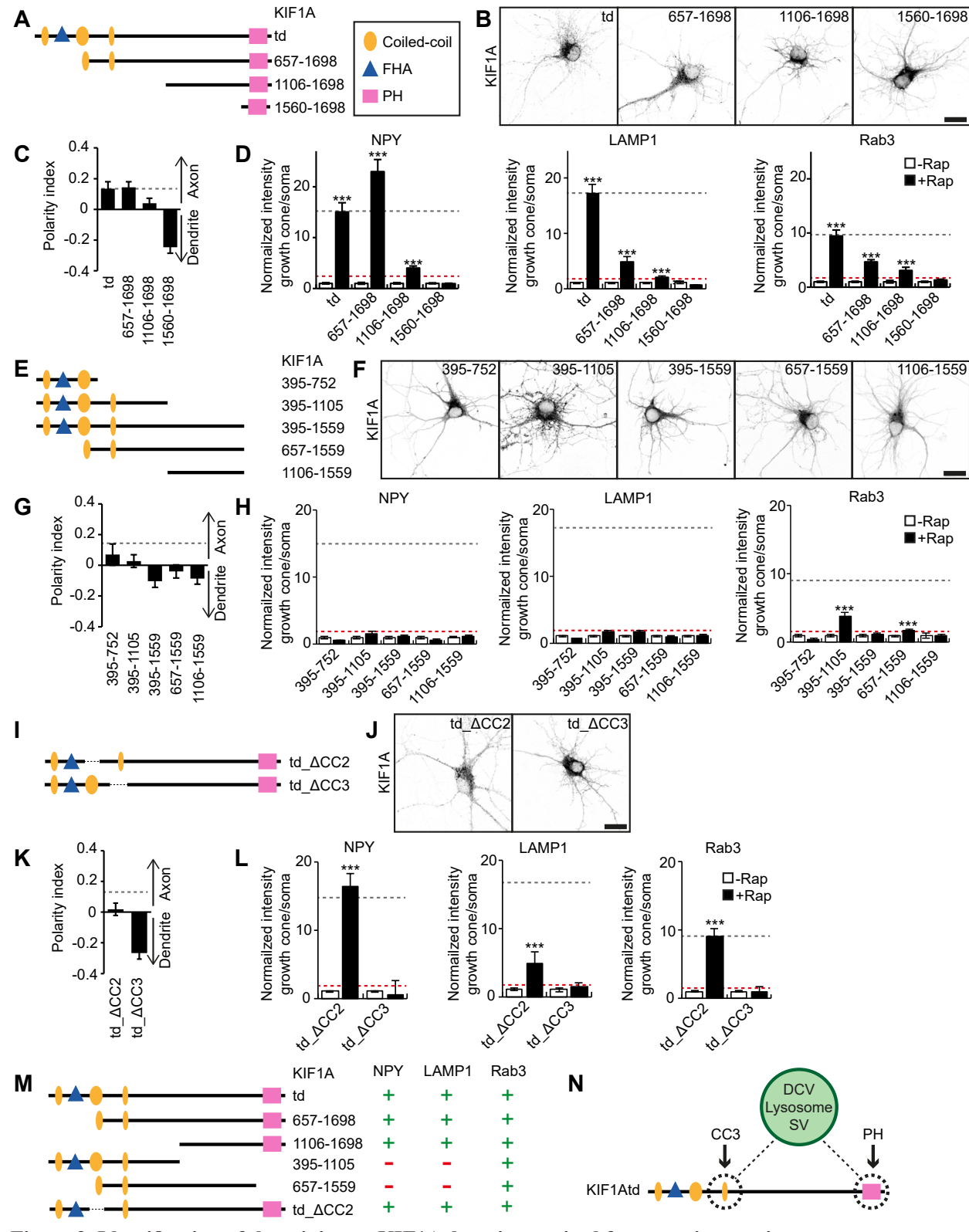

Figure 2. Identification of the minimum KIF1A domain required for cargo interaction Overview of different KIF1Atd fragments, their localization, and interactions with cargo. (A,E,I) Schematic expressing KIF1 Atd truncations (depicted in A, E, and I, respectively). (C, G,K) Quantification of the polarity index of KIF1 Atd truncations (depicted in A, E, and I, respectively). Dotted gray line marks the polarity index of KIF1 Atd $(\mathrm{N}=3, \mathrm{n}=32-40)$. (D, H,L) Quantification of the normalized ratio of cargo intensity in axonal tips to that of the som of hippocampal neurons coexpressing FRB-3myc-fused truncated KIF1Atd constructs (depicted in A, E, and I, respectively), marker-GFP, and FKBP-mRFP-KIF5Cmd, without or with addition of $1 \mu \mathrm{M}$ rapalog. Dotted gray line marks the normalized intensity for KIF1Atd with rapalog treatment. Dotted red line marks the baseline of $5 \%$ of the KIF1Atd signal $(\mathrm{N}=1, \mathrm{n}=7-15)$. (M) Overview of the identified interactions between truncated KIF1Atd and cargo. (N) Schematic depiction showing the interaction between KIFIACC3 and PH domain with DCVs, lysosomes, and SVs. Data are displayed as mean \pm SEM. Mann-Whitney U test; ***, $\mathrm{P}<0.001$. Scale bars: $20 \mu \mathrm{m}$. 

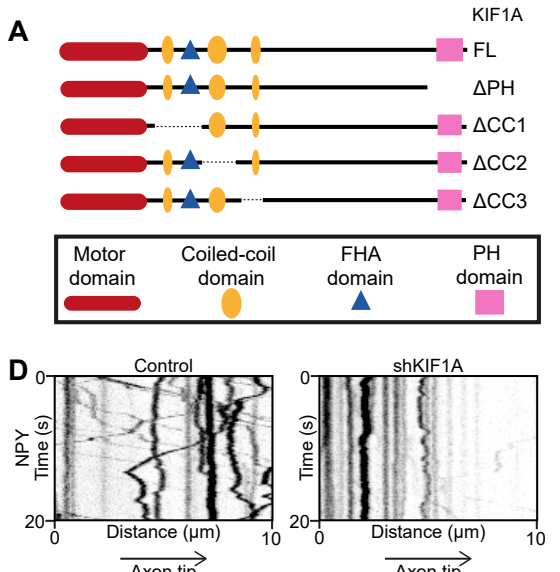

E ${ }_{100 \%}$

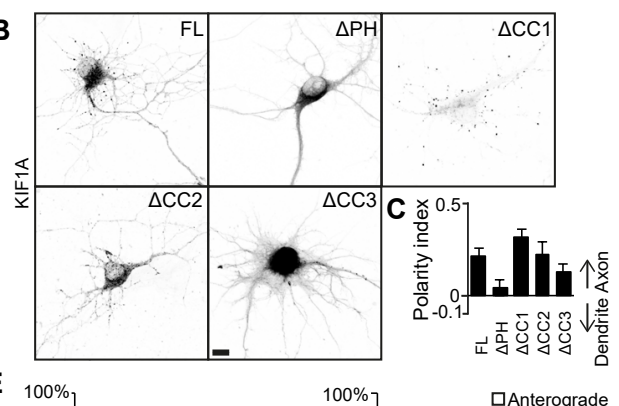

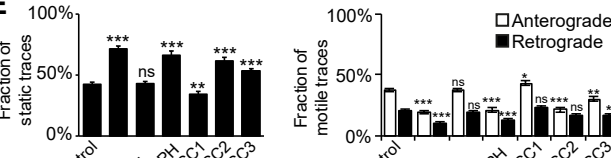

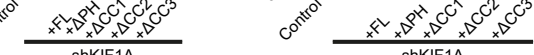
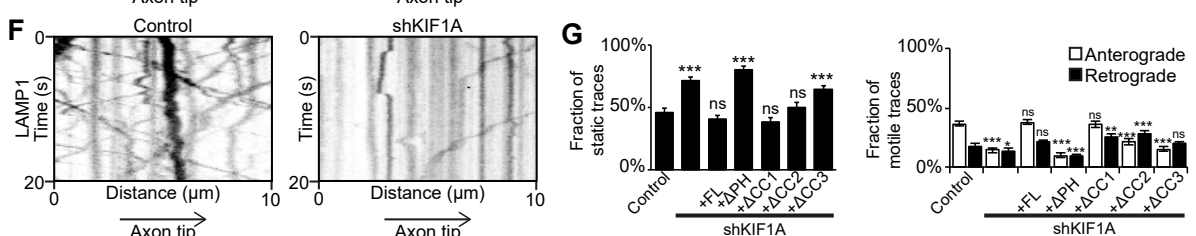

.
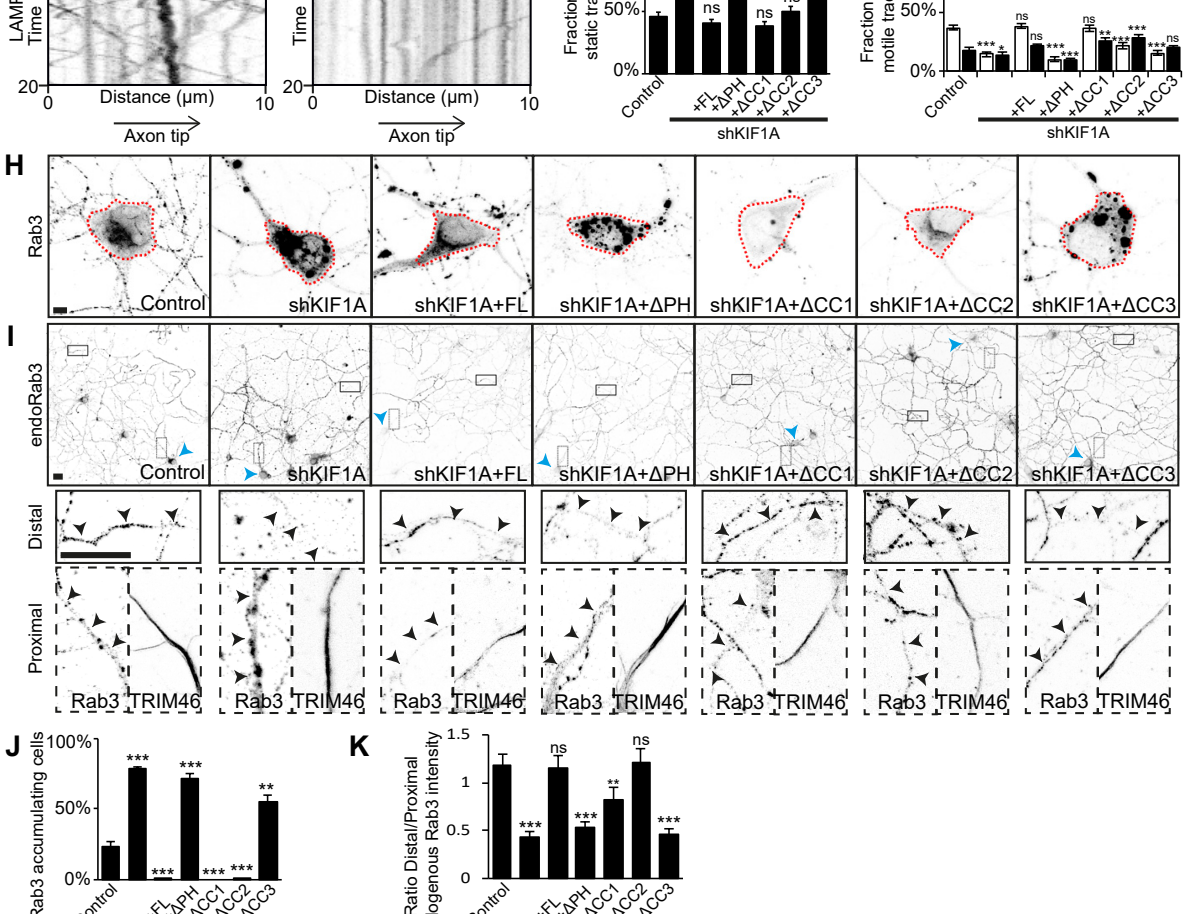

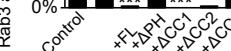

Figure 3.Validation of the KIF1A CC3 and PH domains as minimal domains for cargo interaction KIF1A KD and rescue experiments to verify the requirement of KIF1A CC3 and PH domains in cargo association (A) Schens expressing KIF1 A deletion constructs. (C) Quantification of the polarity index of KIF1 A deletion constructs $(\mathrm{N}=3, \mathrm{n}=39-45)$. (D,F) Representative kymographs showing movement of NPY (D) or LAMP1 (F) vesicles in the AIS in control or when transfected with KIF1A shRNA. (E,G) Quantification of the fraction of static, anterograde, and retrograde NPY (E) or LAMP1 (G) traces in control, KIF1A KD, and rescue with KIF1A deletion constructs (N=3, $\mathrm{n}=40-45$; except $\mathrm{NPY}$ in $\mathrm{KD}+\Delta \mathrm{PH}, \mathrm{N}=2, \mathrm{n}=25$ ). (H) Representative images of the soma of hippocampal neuron expressing GFP-Rab3 in control, cotransfected with KIF1A shRNA, or with KIF1A shRNA and KIF1A deletion constructs. Dotted red line marks the cell soma. (I) Representative images of hippocampal neurons immunostained for Rab3 in control situation, when transfected with KIF1A shRNA, or with KIF1A shRNA and KIF1Adeletion constructs.
Blue arrowheads indicate the soma of a transfected cell, closed boxes mark part of the distal axon, and dotted boxes mark the proximal axon. Zooms of the boxes are shown underneath, including TRIM46 staining in the proxima axon. Black arrowheads indicate the axon of the transfected neuron. (J) Quantification of the number of cells with 400 ). (K) Quntifition of the ratio of endogenous Rab3 intensity in the dista axon to that of the control, KIF1A KD, and rescue with KIF1A deletion constructs $(\mathrm{N}=3, \mathrm{n}=38-40)$. Data are displayed as mean $+\mathrm{SEM}$. Unpaired $\mathrm{t}$ test (E), Mann-Whitney $\mathrm{U}$ test $(\mathrm{G}, \mathrm{J}, \mathrm{K})$; *, $\mathrm{P}<0.05$ ***, $\mathrm{P}<0.01$ ***, $\mathrm{P}<0.001$. Scale bars: $10 \mu \mathrm{m}$ (B), $5 \mu \mathrm{m}(\mathrm{H}), 20 \mu \mathrm{m}(\mathrm{I})$.

The axonal transport of endogenous Rab3 vesicles was rescued by KIF1A_FL, KIF1A $\Delta \mathrm{CC} 1$, and KIF1A $\Delta \mathrm{CC} 2$, but not by KIF1A $\Delta \mathrm{PH}$ or KIF1A $\Delta \mathrm{CC} 3$ (Fig I,K). Thus, the $\mathrm{PH}$ and $\mathrm{CC} 3$ domains are required for KIF1A-mediated trafficking of DCVs, lysosomes, and SVs.

Interaction of KIF1A with cargo via $\mathrm{PH}$ and $\mathrm{CC} 3$ is needed for motor activity To further show the role of the $\mathrm{PH}$ and $\mathrm{CC} 3$ domain of KIF1A for vesicle interaction, we investigated live movement of KIF1A deletion constructs together with DCVs. KIF1A_FL colocalizes and moves together with NPY-containing DCVs (Fig 4A), and similar comovement was observed for KIF1A $\triangle \mathrm{CC} 2$ (Fig 4C). During live imaging, $\sim 50-60 \%$ of NPY vesicles colocalized with KIF1A FL and KIF1A $\triangle \mathrm{CC} 2$ motors (Fig S4B). In contrast, KIF1A $\Delta$ PH, and KIF1A $\triangle \mathrm{CC} 3$ showed diffuse localization and did not colocalize with DCVs. Interestingly, we observed that upon their expression, DCV movements were abolished, indicating that they might act as dominant negatives (Fig 4B,D). As the CC1 is involved in regulation of motor activity, removal of $\mathrm{CC} 1$ results in a constitutively active kinesin motor, which accumulates in distal tips, making it challenging to live image its movement (Huo et al., 2012). We did observe that NPY vesicles are still motile when coexpressed with KIF1A $\Delta \mathrm{CC} 1$ Furthermore, DCVs, lysosomes, and SVs accumulate and colocalize with KIF1A $\triangle C C 1$ i distal tips, indicating an interaction between the cargos and KIF1A $\triangle \mathrm{CC} 1$ (Fig 4E; $\overline{\text { Fig S4D). }}$ When we expressed KIF1A deletion constructs in COS7 cells, we observed that KIF1A FL, KIF1A_ $\triangle \mathrm{CC} 1$, and KIF1A_ $\triangle \mathrm{CC} 2$ translocate into the cell periphery, indicating that they are active motors, which is consistent with our live-imaging experiments in neurons. For KIF1A_ $\triangle \mathrm{CC} 1$, we observed a large accumulation of the motor in the cell periphery, confirming its high motor activity. In contrast, KIF1A_ $\Delta \mathrm{PH}$ and KIF1A_ $\Delta \mathrm{CC} 3$ showed a diffuse localization pattern (Fig S4C). Together, these results suggest that interaction of the KIF1A PH and CC3 domains with cargo is crucial for KIF1A motor activity.

Dimerization of KIF1A via its CC2 domain is important for cargo transport Previously, the $\mathrm{CC} 2$ domain of KIF1A has been implicated in motor dimerization (Hammond et al., 2009). In our KD and rescue experiments, we observed that KIF1A $\Delta C C 2$ was not able to rescue the reduced NPY motility in KIF1A KD, and we speculated that this might be due to a reduced ability of monomer KIF1A to interact with vesicles. To investigate the involvement of different KIF1A domains in dimerization, we expressed GFP-fused domain constructs in HEK293T cells and analyzed cell lysates by normal and native SDS-PAGE. Results show an additional band for the CC2 domain, which is twice its normal size on native gel, suggesting that the domain is dimerized (Fig S4E,F). This was not observed for any of the other domains, suggesting that the $\mathrm{CC} 2$ domain of KIF $1 \mathrm{~A}$ is responsible for motor dimerization. Next, we generated a construct in which we replaced the $\mathrm{CC} 2$ domain with a GCN4 leucine zipper domain (O'Shea et al., 1991), thereby artificially dimerizing the motor (Fig 4F). Expression of the artificially dimerized KIF1A motor in neurons showed that it accumulates in distal tips, while maintaining a slightly axonal polarity comparable to KIF1 A FL (Fig 4H,I; Fig S4H). When we expessed KIF1A $\triangle \mathrm{CC} 2 \mathrm{CCN} 4$ toger wer that the motor colocalized with vesicles in distal tips, suggesting that the motor interacts with DCVs, lysosomes, and SVs (Fig 4G; Fig S4G). Next, we performed KIF1A KD experiments and found that dimerized KIF1A could rescue the NPY motility phenotype (Fig 4J). These data show that KIF1A dimerization by CC2 is important for the regulation of cargo transport. 


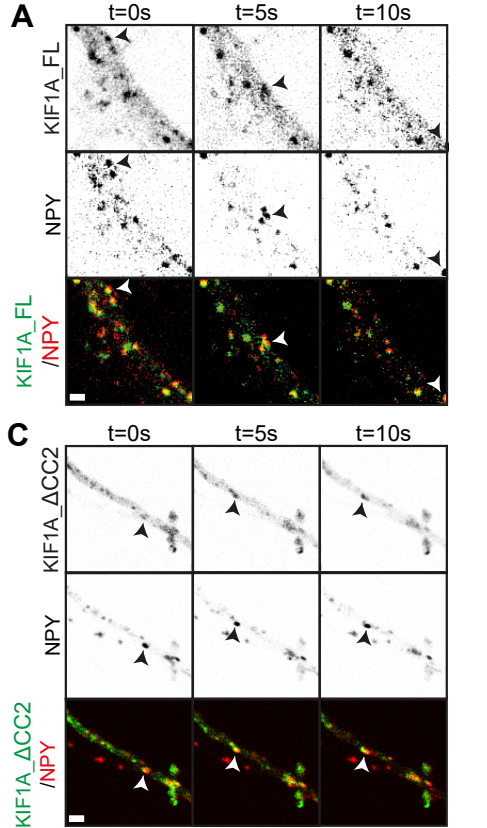

B

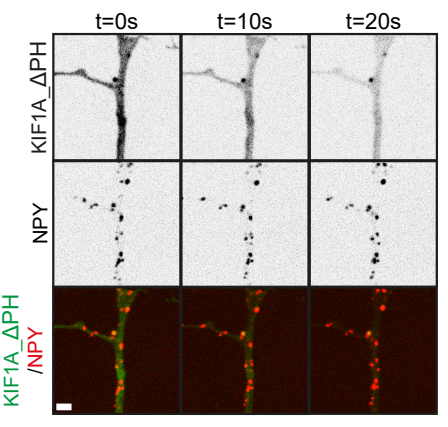

D
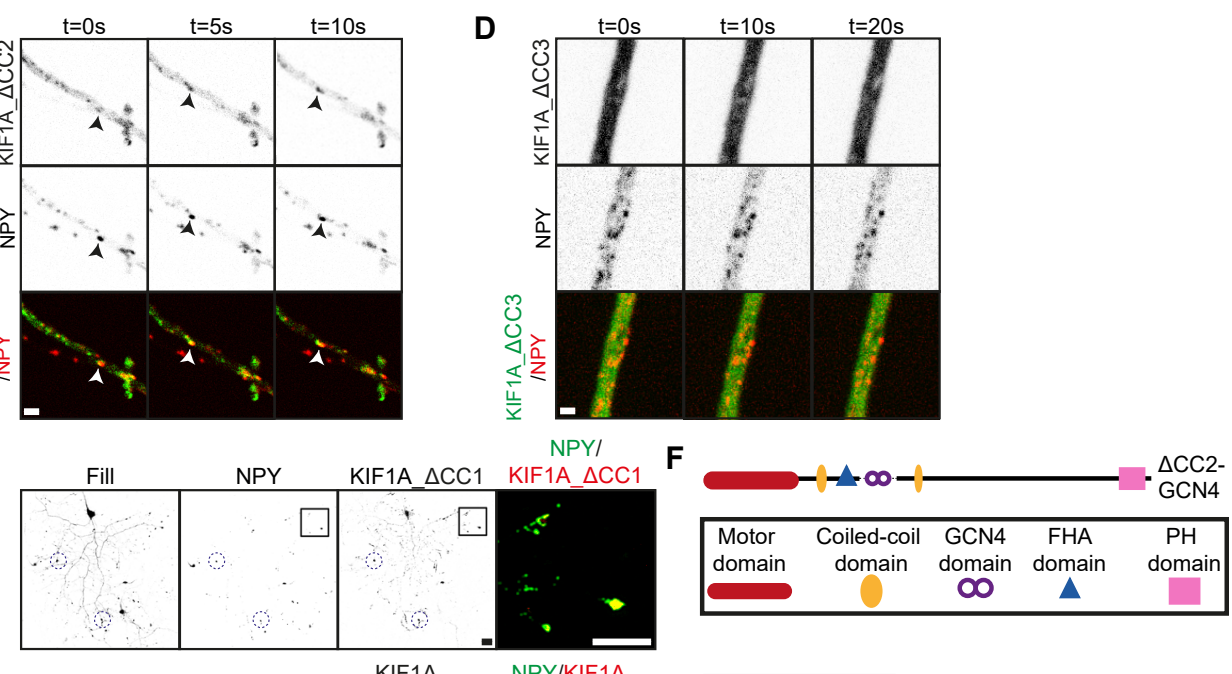

G
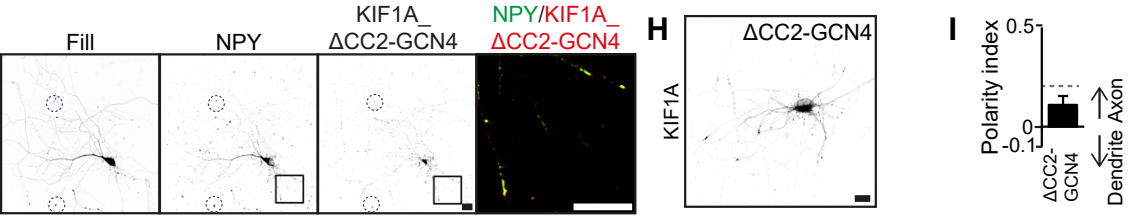

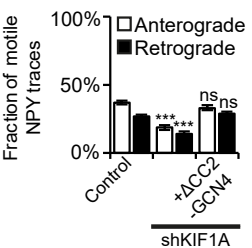

ShKIF1A

$$
\overline{\text { ShKIFIA }}
$$

Figure 4. KIF1A vesicle association and motor dimerization are required for vesicle transport Comovement of KIF1A with cargo and investigation of the involvement of KIF1A dimerization in cargo transport. (A-D) Stills showing movement of KIFIA_FL (A), KIF1A_ $\Delta$ PH (B), KIF1A_ $\Delta C C 2$ (C), or KIF1A $\Delta$ CC
(D) and NPY vesicles in the axon. Arrowheads mark a moving NPY vesicle colocalizing with KIF1A FL (A) or KIF1A $\triangle \mathrm{CC} 2$ (C). (E) Representative image of a hippocampal neuron coexpressing KIF1A $\triangle \mathrm{CC} 1$ and NPY. Blue dotted circles indicate examples of distal tips. Merged image of the boxed region is shown on the right. (F) Schematic depiction of the structure of the KIF1A_ $\Delta$ CC2-GCN4 motor. (G) Representative image of a hippocampal neuron coexpressing KIF1A_ $\triangle \mathrm{CC} 2-\mathrm{GCN} 4$ and NPY. Blue dotted circles indicate examples of distal tips. Merged image of the boxed region is shown on the right. (H) Representative image of a hippocampal neuron expressing KIF1A_ $\triangle \mathrm{CC} 2-\mathrm{GCN} 4$. (I) Quantification of the polarity index of KIF1A_ $\triangle \mathrm{CC} 2-\mathrm{GCN} 4$ motor $(\mathrm{N}=3, \mathrm{n}=38)$. (J) Quantification of the fraction of static, anterograde, and retrograde NPY traces in control, KIF1A KD, and rescue with $\mathrm{KIF} A \mathrm{~A}-\overline{\mathrm{A}} \mathrm{CC}-\mathrm{GCN}(\mathrm{N}=3, \mathrm{n}=35)$. Data are displayed as mean \pm SEM. Unpaired $t$ test, ${ }^{* *}, \mathrm{P}<0.001$. Scalc

KIF1A-cargo binding is regulated by phosphorylation

KIF1A-associated neurological disorder is a neurodegenerative disorder characterized by symptoms including spastic paraplegia, ataxia, and intellectual disability. Several mutation in the KIF1A gene were found to underlie the neurodegenerative symptoms, most of which are found in the KIF1 A motor domain. Five mutations in the human KIF1 Atd have been described, one of them being the mutation of serine 1758 to glutamine, which localizes in the PH domain (Gabrych et al., 2019; Rivière et al., 2011). We reasoned that this mutation might disrupt the KIF1A-cargo interaction. Human S1758 corresponds to S1665 in our mouse cDNA, and we therefore mutated S1665 to Q1665 in KIF1A_1106-1698, the minimal KIF1A fragment that associates with cargo (Fig 5A). The interaction of KIF1A_S1665Q with DCVs and SVs was then assessed in our motor assay, and we found that it was unable to transport DCV or SVs, indicating that this mutation indeed interferes with KIF1A-cargo binding (Fig 5B). As the mutation involved the change from a serine, a potential phosphorylation site, to a glutamine, phon inveriont role in the KIF1A-cargo interaction. Therefore, we generated KIF1A S1665D, containing a phosphomimetic aspartic acid residue. Using this construct, we observed that transport of DCVs and SVs was restored to levels comparable to KIF1A 1106-1698, suggesting that phosphorylation of serine 1665 is required for an interaction between KIF1A and cargo. Previously, it was shown that KIF1A has a calmodulin (CaM) binding site and that activation of the motor is calcium dependent (Stucchi et al., 2018). Therefore, we hypothesized that binding of $\mathrm{CaM}$ activates $\mathrm{Ca}^{2+}$ CaMKII, which then phosphorylates KIF1A. To test this, we generated KIF1A $5 *$ Ala (Fig 5A), which contains five alanine substitutions in the CaMbinding domain of KIF1A 657-1698, thereby preventing the interaction of KIF1A with CaM (Stucchi et al., 2018). In our motor assay, KIF1A $5 *$ Ala was unable to interact with DCVs (Fig $5 \mathrm{C}$ ), suggesting that the interaction of KIF1A with $\mathrm{CaM}$ is needed for vesicle association. We then investigated NPY axonal vesicle entry in our motor assay in the presence of KN-93, a specific CaMKII inhibitor. When cells were treated with KN-93, there was only a small increase in axonal NPY entry after addition of rapalog. In contrast, the negative control, treated with KN-92, behaved like the untreated control condition (Fig 5D). Altogether, these results suggest that phosphorylation of KIF1A by CaMKII, potentially in the PH domain, is an important step in vesicle association.

\section{Identification of adaptors for specific cargo types}

Next, we turned our focus to different KIF1A adaptors. The main adaptors described for KIF1A are liprin- $\alpha$, TANC2, Arl8A/B, and MADD, and they have been implicated in transport of DCVs, lysosomes, and SVs. Using our enginered motor platform, we generated FRB-3mycadaptor constructs and expressed these in neurons together with FKBP-mRFP-KIF5Cmd and GFP-tagged markers. This allowed assessment of the interaction of each adaptor with DCVs, lysosomes, and SVs. Results show that Arl8A and Arl8B both interact with DCVs and lysosomes, whereas MADD clearly interacts only with SVs (Fig 5E). Liprin- $\alpha$ and TANC2 did not interact with any of the vesicle types in our motor assay (Fig 5E).

We further focused our attention on Arl8A/B and MADD and depleted these adaptors in hippocampal neurons. Arl8A and Arl8B shRNA constructs were validated using immunofluorescence (Fig S5A-D), and we used shArl8A-1 and shArl8B-2 for furthe experiments. When coexpressing these shRNA constructs together with LAMP1, we observed that LAMP1 is prevented from entry into the axon and accumulates in the proximal axon (Fig $\mathrm{S} 5 \mathrm{E})$. To circumvent potential redundancy between the two Arl8 proteins, we then performed an Arl8A/B double KD and found a decrease in both NPY and LAMP1 motility (Fig 5F-I), supporting a role for Arl8 in transport of these vesicles. When we knocked down MADD using a previously validated shRNA (Niwa et al., 2008), we observed changes in the localization pattern of overexpressed Rab3-GFP. In control situations, it has a punctate pattern, but it becomes diffuse upon MADD depletion, suggesting loss of its vesicle association (Fig 5J) 

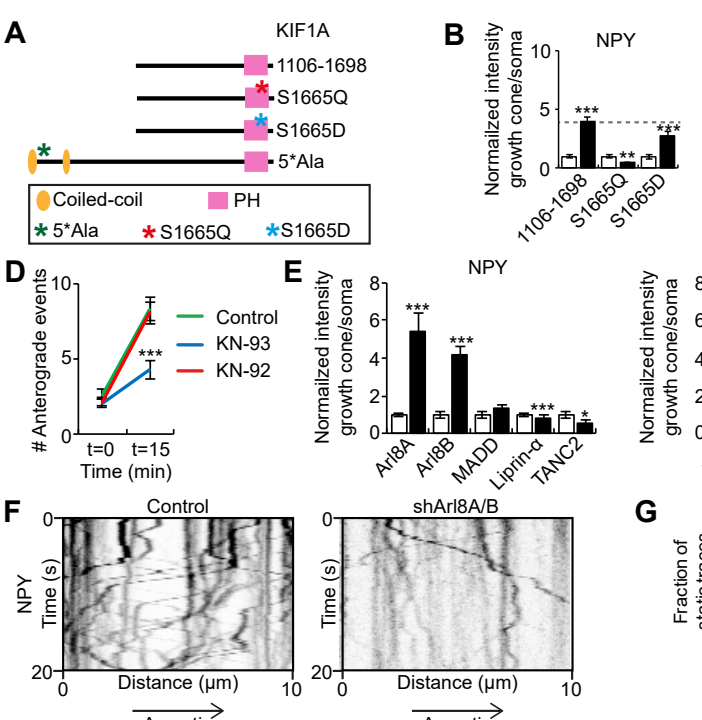

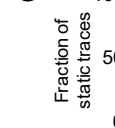

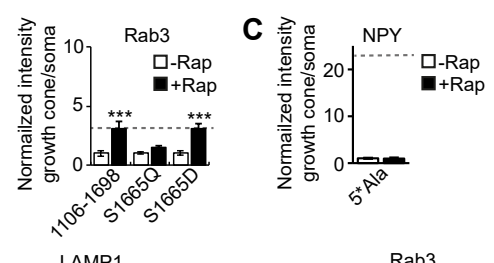

LAMP1
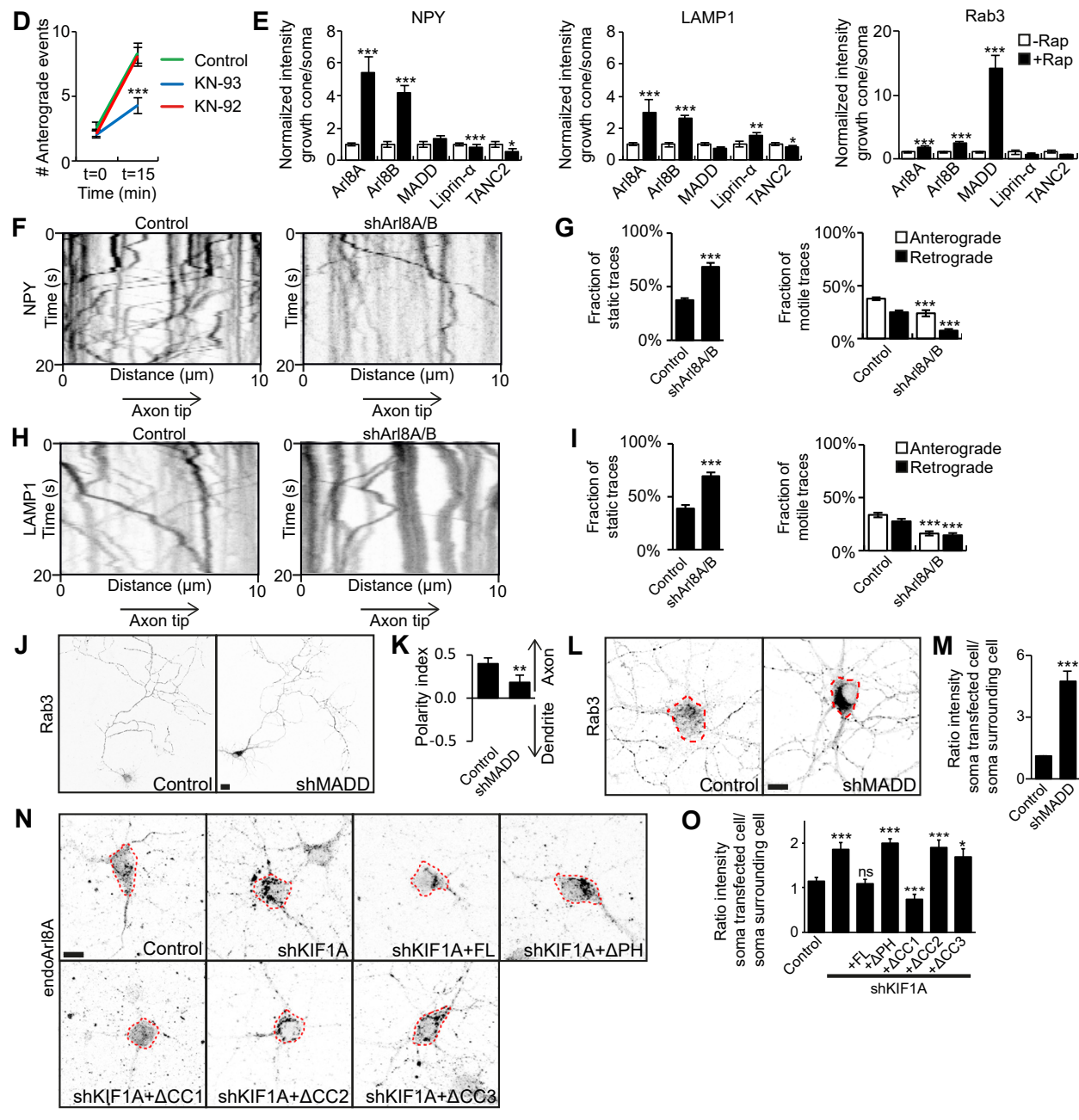

0 言 $\overline{\mathrm{o}}$

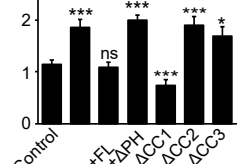

shKIF $1 A+\Delta C C 1$ shKiF $1 A+\triangle C C 2$ sh́KIF $1 A+\Delta C C_{3}$

Figure 5. Identification of KIF1A adaptors for specific cargo transport

Identification of a phosphorylation mechanism in KIF1A-cargo binding and Arl8A and MADD as adaptors regulating KIFIA specificity for DCVs and yysosomes or SV, respectively. (A) Schematic depiction of the structur of KIFIA_1106-1698 mutated constructs and KIFIA_5*Ala. (B,C) Quantification of the normalized ratio of cargo intensity in axonal tips to that of the soma of hippocampal neurons coexpressing FRB-3myc-fused mutated $1 \mu \mathrm{M}$ rapar with rapalog treatment $(\mathrm{N}=1, \mathrm{n}=15)$. (D) Quantification of the axonal entries of $\mathrm{NPY}$ vesicles before and 15 min after addition of $1 \mu \mathrm{M}$ rapalog in neurons expressing FRB-3myc-KIF1Atd, NPY-GFP, and FKBP-mRFP-KIF5Cmd in control or after treatment with $1 \mu \mathrm{M} \mathrm{KN}-93$ or $1 \mu \mathrm{M} \mathrm{KN}-92$ for $20 \mathrm{~h}(\mathrm{~N}=3, \mathrm{n}=30)$. (E) Quantification of the normalize ratio of cargo intensity in axonal tips to that of the soma of hippocampal neurons coexpressing FRB-3myc-fuse adaptors, cargo-GFP, and FKBP-mRFP-KIF5Cmd, without or with addition of $1 \mu \mathrm{M}$ rapalog $(\mathrm{N}=1, \mathrm{n}=10-15)$. (F,H) Representative kymographs showing movement of NPY (F) or LAMP1 (H) vesicles in the AIS in control or when transfected with Arl8A/B shRNA. (G,I) Quantification of the fraction of static, anterograde, and retrograde NPY (G)

or LAMP1 (I) traces in control or when transfected with Arl8A/B shRNA ( $\mathrm{N}=3, \mathrm{n}=29-30)$. (J) Representative images of hippocampal neurons expressing GFP-Rab3 in control or when cotransfected with MADD shRNA. (K) Quantification of the polarity index of GFP-Rab3 in control or MADD KD ( N=3, n=29-30). (L) Representative images of the soma of hippocant the cell som. (M) Quntifeation of the ratio of whold transfected cell to that of a surrounding cell in control or MADD KD ( $=3, n=29-30)$. (N) Representative in of the soma of hippocampal neurons immunostained for Ar18A in control, when cotransfected with KIF1 A shRNA or with KIF1A shRNA and KIF1A deletion constructs. Dotted red lines mark the cell soma. (O) Quantification of the ratio of endogenous Arl8A intensity in a transfected cell to that of a surrounding cell in control, KIF1A KD, and rescue with KIF1A deletion constructs ( $N=3, n=26-30$ ). Data are displayed as mean \pm SEM. Unpaired test $(\mathrm{G}, \mathrm{I}, \mathrm{K})$ and Mann-Whitney U test (B-E,M,O), *, P $<0.05$; **, $\mathrm{P}<0.01$; ***, P<0.001. Scale bars: $10 \mu \mathrm{m}(\mathrm{L}, \mathrm{N}), 20 \mu \mathrm{m}$ (J). In addition, polarity index quantifications show that Rab3-GFP localizes more somatodendritically when MADD is depleted (Fig $5 \mathrm{~K}$ ). We also looked at endogenous Rab3 and found that it accumulates in the cell soma upon MADD KD (Fig 5L,M). These findings show that Arl8A/B are adaptors for DCVs and lysosomes, and MADD is an adaptor for SVs.

\section{Arl8A and MADD mediate the interaction between KIF1ACC 3 and specific cargo} We further explored the interaction between the adaptors and KIF1A. First, we expressed Arl8A and Arl8B in COS7 cells and found that Arl8A is diffuse over the cell, whereas Arl8B is mainly diffuse but also has some accumulation in the cell periphery (Fig S5F). When we coexpressed these adaptors together with KIF1A_FL, a clear relocalization of Arl8A into the cell periphery was observed, where it colocalized with KIF1A. As Arl8B already localized in the cell periphery, we could not confirm that the observed colocalization with KIF1A was due to an interaction with the motor (Fig S5G). To further investigate the interaction between KIF1A and adaptors, we performed pull-down experiments where we used the adaptors to pull down KIF1A 395-1105. Western blot analysis confirmed an interaction of ( $395-1105$ with Arl8A and MADD, whereas no interaction was seen with Arl8B (Fig $\mathrm{S} 5 \mathrm{H}, \mathrm{I})$ These data point to a role for the $\mathrm{CC} 3$ domain in the interaction with adaptors. To confirm the observation that Arl8A, but not Arl8B, is an adaptor for KIF1A-mediated DCV and lysosome transport, the localization of Arl8A was analyzed in KIF1A-depleted neurons, and we observed that endogenous Arl8A accumulates in the cell body in KIF1A KD. Rescue experiments using different KIF1A deletion constructs (Fig 3A) show that this phenotype was rescued by KIF1A_FL and KIF1A_ $\Delta \mathrm{CC} 1$, but not by KIF1A_ $\Delta \mathrm{PH}$, KIF1A_ $\Delta \mathrm{CC} 2$, or KIF1A $\triangle \mathrm{CC} 3$ (Fig 5N,O). This effect resembles the pattern observed in rescue experiments of the NPY motility phenotype (Fig 3E), suggesting that Arl8A is an adaptor for KIF1Amediated DCV transport. In addition, when LAMP1 was coexpressed in KIF1A-depleted neurons, we observed that the Arl8A accumulation colocalizes with LAMP1-containing lysosomes, supporting a role for Ar18A in lysosomal transport by KIF1 A (Fig S5J). In contrast, no changes in endogenous Arl8B localization were observed upon KIF1A depletion (Fig S5K,L). Altogether, these data suggest that Arl8A and MADD interact with KIF1A via its CC3 domain and that Arl8A specifically is involved in the association between KIF1A and DCVs and lysosomes, whereas MADD specifically mediates the interaction between KIFIA and SVs.

\section{DISCUSSION}

The three KIF1A CC domains play distinct roles in regulating motor activity Kinesin motors contain different structural domains that contribute to their regulation and function. The stalk domain of KIF1A contains three CC domains and an FHA domain between $\mathrm{CC} 1$ and $\mathrm{CC} 2$. Previous studies have described different roles for these domains in motor regulation. For instance, $\mathrm{CC} 1$ was found to regulate motor activity by inhibition of the motor domain (Ren et al., 2018; Huo et al., 2012). In addition, both CC1 and CC2 have been implicated in motor dimerization (Huo et al., 2012; Hammond et al., 2009). Also, the FHA domain was found to be involved in the regulation of motor activity, suggesting that the central CC1-FHA-CC2 region might function as a negative regulatory center for KIF1A motor activity (Lee et al., 2004; Huo et al., 2012). 
Here, we found that removal of $\mathrm{CC} 1$ results in a very processive motor, which in neurons accumulates in distal tips. This localization is comparable to the localization of only the KIF1A motor domain (Huang and Banker, 2012), thereby supporting a role for CC1 in motor inhibition. Furthermore, we demonstrate that $\mathrm{CC} 2$ is involved in motor dimerization. Although removal of $\mathrm{CC} 2$ does not completely abolish cargo trafficking, we artificially dimerized KIF1A accumulates rapidly in distal tips, suggesting that dimerization of KIF1A is important for its activity. Lastly, our results show that the KIF1A CC3 is required for vesicle interaction. This is in line with previous work, which has suggested that the stalk region of KIF1A is critically important for cargo interaction (Klopfenstein and Vale, 2004; Xue et al., 2010; Guardia et al., 2016). Interestingly, removal of the CC3, thereby abolishing cargo interaction, resulted in a nonprocessive motor, suggesting that kinesin-cargo interactions could be required for motor activity. Overall, our data point to a role for all three $\mathrm{CC}$ domains in motor activity, however via various mechanisms.

KIF1A PH domain binding to vesicular cargo is regulated by phosphorylation The C-terminal PH domain is a unique feature of KIF1A and KIF1B $\beta$ motors and has been implicated in linking these motors to cargo. The PH domain of KIF1A has been shown to bind to PIP2 on vesicle membranes and mediates efficient vesicle transport in different cell types (Klopfensteir confirm that the PH domain of KIF1A associates with DCVs, lysosomes, and SVs in living neurons, indicating that cargo binding to the $\mathrm{PH}$ domain is a conserved mechanism across cell types. We identified S1665 in the PH domain as a crucial residue for cargo interaction, an by using phosphodeficient S1665Q and phosphomimetic S1665D mutants, we showed tha phosphorylation of this residue is essential. One could speculate that phosphorylation of serine residue enhances binding with phosphoinositides on the cargo membrane. We also foun that cargo interaction is dependent on CaMKII activity. Interestingly, $\mathrm{Ca}^{2+} / \mathrm{CaM}$-regulated kinesin motors have been engineered, and they revealed that $\mathrm{Ca}^{2+}$ levels regulate kinesin motor activity (Shishido and Maruta, 2012). In addition, phosphorylation-dependent cargo loading and trafficking has been described for other kinesin motors, such as KIF3A (Ichinose et al., 2015), and a $\mathrm{Ca}^{2+}$ regulation mechanism was found for mitochondrial trafficking by kinesin-1 (Wang and Schwarz, 2009). Furthermore, it has been shown that KIF1A activity is kinesin-1 (Wang and Schwarz, 2009). Furthermore, it has been shown that KIF1A activity is of DCVs (Stucchi et al., 2018). It is tempting to speculate on a phosphorylation-dependen mechanism for regulating KIF1A-cargo transport, where binding of CaM to KIF1A activate CaMKII to phosphorylate the PH domain and allow cargo interaction. This model fits well regulating KIF1A cargo trafficking.

Cargo binding is stabilized by interaction with specific adaptors

Cargo-adaptor proteins are known to regulate kinesin-cargo interactions (Hirokawa et

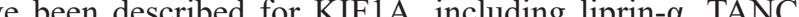

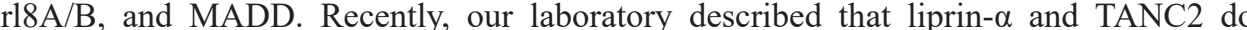
not act as classic motor-cargo adaptors but rather as signposts to recruit KIF1A-cargo complexes at synaptic sites (Stucchi et al., 2018). Using engineered motors, we show that liprin- $\alpha$ and TANC2 do not interact with vesicles, thereby supporting their role as signposts rather than classic cargo adaptors. Arl8A and Arl8B have been implicated in transport of lysosomes and SVs across different cell types (Niwa et al., 2016; Farías et al., 2017; Rosa-Ferreira and Munro, 2011). Furthermore, a direct interaction between the KIF1A CC3 domain and Arl8A was established by affinity chromatography and yeast twohybrid assays (Wu et al. 2013). MADD has been described as an adaptor for KIFIA- and KIF1B - mediated transport of SVs, and it was shown to interact with the KIF1B $\beta$ stalk (N) support a role of the stalk domain in the interaction between KIF1A and its adaptors.
Interestingly, KIF1A_657-1105 was also identified as the binding region for the nonclassic adaptors liprin- $\alpha$ and TANC2 (Stucchi et al., 2018), suggesting that adaptors with different functions associate with the same region in KIF1A. Based on our data and previous literature, we propose a general mechanism for KIF1A-mediated cargo trafficking. In its autoinhibited state, inactive KIF1A exists as a dimer (Hammond et al., 2009). This dimeric conformation is established by the $\mathrm{CC} 2$ domain. An increase in the local $\mathrm{Ca}^{2+}$ level activates $\mathrm{CaM}$ and induces its binding to KIF1A, thereby diminishing KIF1A autoinhibition by $\mathrm{CC} 1$ and opening the motor domain. In parallel, CaM activates CaMKII, which phosphorylates the PH domain of active KIF1A and enables cargo binding. The KIF1A-cargo association is stabilized by specific adaptors, including Arl8A and MADD, which interact with both the cargo and the KIF1A CC3 domain. KIF1A transports the cargo until it reaches an adaptor signpost, such as liprin- $\alpha$ and TANC2, which outcompetes the classic adaptor for binding with the KIF1A stalk domain. This decreases the strength of the KIF1A-cargo interaction and results in release of the vesicle in the designated area.

In summary, we have dissected the role of different $\mathrm{CC}$ domains in KIF1A motor activity and show that $\mathrm{CC} 1$ regulates autoinhibition, $\mathrm{CC} 2$ regulates motor dimerization, and CC3 is involved in cargo interaction. Furthermore, we propose a two-step model for KIF1Acargo binding and transport in neuronal cells. First, phosphorylation of S1665 in the KIF1A $\mathrm{PH}$ domain may enable binding to any cargo vesicle, whether it is DCVs, lysosomes, or SVs. Specificity for a cargo is achieved in a second step in which a specific adaptor stabilizes the interaction of KIF1A with a certain cargo. The adaptor interacts with both the cargo and the CC3 domain of KIF1A. We identified Arl8A as specific adaptor for DCVs an lysosomes and MADD as a specific adaptor for SVs. This mechanism fits well in the model for specific KIF1A-mediated cargo transport and delivery. As many similarities have been found in KIF1A vesicle transport in different cell types and between KIF1A and KIF1B $\beta$, the described mechanism may be a generic model for KIF1-mediated cargo trafficking.

\section{AUTHOR CONTRIBUTIONS}

J.J.A. Hummel designed, performed, and analyzed the experiments, processed data for representation in the figures, and wrote the manuscript. C.C. Hoogenraad supervised the research, coordinated the study, and edited the manuscript.

\section{MATERIALS AND METHODS}

All animal experiments were approved by the Dutch Animal Experiments Committee and were performed in line with guidelines of Utrecht University, Dutch law (Wet op de Dierproeven, 1996), and European regulation (Guideline 86/609/EEC). In this study, neurons obtained from embryonic day 18 stage embryos of both genders from female pregnant Wistar rats (Janvier) were used. Pregnant rats were at least $10 \mathrm{wk}$ old and not involved in previous experiments. Animals were housed in transparent Plexiglas cages with wood-chip bedding and paper tissue and provided with unrestricted access to food and water. Adult rats were kept with a companion and kept in a 12 light-dark cycle with a temperature of $22 \pm 1^{\circ} \mathrm{C}$

Primary hippocampal neuron cultures and transfections

Primary hippocampal neuron cultures were prepared from embryonic day 18 rat brains according to protocols described previously (Kapitein et al., 2010c). Cells were plated at a density of $100 \mathrm{~K}$ neurons per well in a 12 -well plate on coverslips coated with poly-L-lysine ( $37.5 \mu \mathrm{g} / \mathrm{ml}$; Sigma-Aldrich) and laminin $(1.25 \mu \mathrm{g} / \mathrm{ml}$; Roche) an gM lutamate (Sigma-Aldrich), and $1 \%$ penicillin/streptomycin (Gibco) at $37^{\circ} \mathrm{C}$ and $5 \% \mathrm{CO}$

Hippocampal neurons were transfected at the indicated time points using Lipofectamine 2000 (Invitrogen).

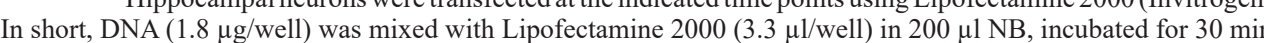
and added to neurons in transfection medium (NB supplemented with $0.5 \mathrm{mM}$ glutamine) for $45 \mathrm{~min}$ at $37^{\circ} \mathrm{C}$ and $5 \%$ $\mathrm{CO}_{2}$. Neurons were then washed with NB and transferred to their original medium at $37^{\circ} \mathrm{C}$ and $5 \% \mathrm{CO}_{2}$ until fixation at indicated time points. For engineered motor experiments, neurons were transfected at 7 days in vitro (DIV7) When used for analysis in fixed cells, rapalog (final concentration of $1 \mu \mathrm{M}$ ) was added directly after transfection, an neurons were fixed on DIV8. When used for live-imaging experiments, neurons were imaged on DIV8. For KIFIAcargo or cargo-cargo colocalization experiments, neurons were transfected on DIV3 and fixed or imaged on DIV4. 
To calculate the polarity indices, neurons were transfected on DIV7 and fixed on DIV8. For KD experiments, neurons were transfected on DIV4 and fixed or imaged on DIV8.

Cultured cells and transfections

HEK293T and COS7 cells were cultured in 50/50 DMEM/Ham's F10 (Lonza) medium supplemented with 10\% FCS (Sigma-Aldrich) and 1\% penicillin/streptomycin (Sigma-Aldrich). HEK293T cells were transfected using MaxPEI (Polysciences) in a ratio of 3:1 PEI:DNA, according to the manufacturer's protocol. COS7 cells were plate on 18-mm glass coverslips 1d before transfection with DNA constructs using FuGENE6 (Roche) and following the manufacturer's protocol.

\section{DNA and shRNA constructs}

The following DNA constructs used in this study were described before: $p \beta$-actin-HA- $\beta$-galactosidase (Hoogenraad et al, 2017), GFP-Rab3C (van Vlijmen et al 2008), TfR-GFP (Buck et al, 2000), and BirA coding vector and pebioGFP (van der Vart ( 2013). GW1-KIF5C 1-559-mRFP-(FKBP)2 was ), and Bir coding vector and from pBa-Kif5C 1-559-GFP (plasmid 45059; Addgene) using GW1-PEX-mRFP-(FKBP)2 (Kapitein et al., 2010a) as backbone. GW 1 -FRB-3myc-KIF5A 375-1032 was cloned using a PCR-based Gibson Assembly strategy with
the GW1-HA expression vector as backbone and GW1-GFP-FRB (Kapitein et al., 2010b) and GW1-GFP-KIF5A to generate the GW1-FRB-3myc backbone. GW1-FRB-3myc-KIF1A 395-1698 (KIF1Atd) was cloned using GW1-HA-KIF1A (Kevenaar et al,, 2016) as PCR template into the GW1-FRB-3myc backbone. The following KIF1Atd fragments were generated by PCR using GW1-FRB-3myc-KIF1A_395-1698 as template and were cloned
into the GW1-FRB-3myc backbone: KIF1A 657-1698, KIF1A 1106-1698, KIF1A 1560-1698, KIF1A_395-752, into the GW1-FRB-3myc backbone: KIF1A_657-1698, KIF1A_1106-1698, KIF1A_1560-1698, KIF1A_395-752, KIF1A_395-1106, KIF1A_395-1559, KIF1A_657-1559, KIF1A_1106-1559, KIF1A 395-600 701-1698 (KIF1A (KIF1A $\triangle P H, K I F 1 A \triangle C C 1, K I F 1 A \triangle C C 2$, KIF1A $\triangle C C 3$, and KIF1A $\triangle C C 2$-GCN4) were cloned with PCR-

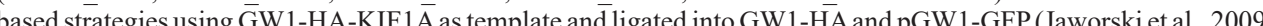
expression vectors. GFP-KIF1 A domain constructs ( $\mathrm{CC1}$, 410-480; FHA, 500-580; $\mathrm{CC} 2,600-700$; and CC3, 781-850) were generated using PCR-based strategies with GW1-HA-KIF1 A as template and pGW1-GFP as backbone. Adaptor were cloned into the GW1-FRB-3myc, bioGFP, and pGW1-GFP backbone using PCR-based strategies with Arl8 A (plasmid 67403; Addgene), Arl8B (plasmid 67404; Addgene), and MADD (IMAGE clone 5271798) as templates For KD experiments, shRNAs were inserted into the pSuper vector (Brummelkamp et al., 2002), which contains a scrambled sequence. The shRNA for KIF1A has been described before (Kevenaar et al., 2016). For Arl8A and Arl8B shRNAs, the following targeting sequences were used: shArr8A-1, 5'-GCGTCAGGACAGTTCAATGshArl8A-2, 5'-TCEACAACCTGCTAGACAA-3'; sh-Arl8A-3, 5-ACC-1, 5'-GGGACTGCTGCTA-3; shArl8B-1 5'-ACCGAGAGATCTGCTGCTA-3'; shArl8B-2, 5'-AGAGGCGTCAATGCAATTG-3'; and shArl8B-3, 5-GAA CCTGTCTGCTATCAA-3. The sequence used for shMADD (5-ATCCTGCCAGAACCGGAAT-3') has bee

\section{Antibodies and reagents}

The following antibodies and dilutions were used in this study for immunofluorescence experiments: mouse anti(1:200, 610379; BD Biosciences), rabbit anti-Arl8 A (1:200, 17060-1-AP; Proteintech), rabbit anti-ArlsB (1:200, 13049-1-AP; Proteintech), rabbit anti-TRIM46 serum (1:500, described before [van Beuningen et al, 2015]), go anti-chicken Alexa Fluor 405 (1:400, ab175675; Abcam), goat anti-rabbit Alexa Fluor 405 (1:400, A31556; Therm Fisher Scientific), goat anti-mouse Alexa Fluor 488 (1:400, A11029; Thermo Fisher Scientific), goat anti-rabb Alexa Fluor 488 (1:400, A11034; Thermo Fisher Scientific), goat anti-mouse Alexa Fluor 568 (1:400, A11031; Thermo Fisher Scientific), goat anti-chicken Alexa Fluor 568 (1:400, A11041, Thermo Fisher Scientific), and goa anti-mouse Alexa Fluor 647 (1.400, A21236, Thermo Fisher Scienticic). For live-imaging analysis, NF-CF555; (Farias et al., 2016) was used. For Western blot, the following antibodies were used. mouse anti-myc (1.200, SC-40; BoR) COR), and goat-anti-rabbit IRDye680LT (1:20,000, 926-68021; LI-COR). Reagents used in this study are rapalo (AP21967, 635056; TaKaRa), KN-93, and KN-92.

\section{Immunofluorescence staining and imaging}

作 RT. Cells were washed three times in PBS-CM ( $\mathrm{PBS}, 1 \mathrm{mM} \mathrm{MgCl} 2$, and $0.1 \mathrm{mM} \mathrm{CaCl}$ ), permeabilized in $0.2 \%$ antibodies were diluted in $0.2 \%$ gelatin and incubated for $30 \mathrm{~min}$ at $37^{\circ} \mathrm{C}$. Next, coverslips were washed three timy in PBS-CM, incubated with secondary antibody diluted in $0.2 \%$ gelatin for 30 min at $37^{\circ} \mathrm{C}$, washed three times in PBS-CM, and mounted in Fluoromount (Invitrogen).

Fixed cells were imaged on (1) a Carl Zeiss LSM 700 confocal laser scanning microscope running ZEN2011 software, using a Plan-Apochromat 40×/1.30 oil differential interference contrast objective or a PlanApochromat $63 \times / 1.40$ oil differential interference contrast objective; or (2) a Nikon Eclipse 80i upright widefiel fluorescence microscope, equipped with a Photometrics CoolSNAP HQ2 charge-coupled device camera and Nikon NIS Br software, using a Plan Fluor $40 \times \mathrm{NA} / 1.30$ oil objective. For quantitative experiments, image settings were kept identical for all images within one experiment.

\section{Pull-down experiments and Western blotting}

or pull-down experiments, HEK293T cells were diluted and plated into six-well plates $1 \mathrm{~d}$ before transfection with with pCl-Neo-BirA, GWI-FRB-3myc-KIF1A_395-1105, and bioGFP-tagged constructs. $24 \mathrm{~h}$ after transfection, X-100, and protease inhibitors [Roche]) on ice for 30 min.er (100 mM Tris HCl, pH 7.5, $150 \mathrm{mM} \mathrm{NaCl}, 1 \%$ Trito krpm at $4^{\circ} \mathrm{C}$. For pull-down experiments, supernatants. were incubated for $1.5 \mathrm{~h}$ at $4^{\circ} \mathrm{C}$ with blocked (incubation for 30 min at $\mathrm{RT}$ in $50 \mathrm{mM}$ Tris $\mathrm{HCl}, \mathrm{pH} 75,150 \mathrm{mM} \mathrm{KCl}$ and $0.2 \mu \mathrm{g} / \mathrm{\mu l}$ chicken egg albumin) Streptavidi Dynabeads M-280 (Invitrogen), after which beads were washed five times with washing buffer (100 $\mathrm{mM}$ Tris $\mathrm{HCl}$ $\mathrm{pH} 7.5,250 \mathrm{mM} \mathrm{NaCl}$, and $0.5 \%$ Triton X-100). Proteins were eluted from beads by boiling for $10 \mathrm{~min}$ at $95^{\circ} \mathrm{C}$ in $2 \times$ DTT+ sample buffer ( $20 \%$ glycerol, 4\% SDS, $200 \mathrm{mM}$ DTT, $100 \mathrm{mM}$ Tris HCl, pH 6.8, and bromophenol blue), and protein samples were run on 10\% SDS-PAGE gels. For SDS-PAGE experiments, lysates from HEK293 cells expressing GFP-KIFIA domain constructs were made as described above. Lysates were either boiled at $95^{\circ} \mathrm{C}$ in $1 \times$ DTT + sample buffer and run on $12 \%$ SDS-PAGE gels or run on 12\% mini-protean TGX precast protein gel (Bio-Rad) without boiling in $1 \times$ DTh- sample buffer. Proteins were then transferred from the gel to nitrocellulose membranes (Bio-Rad) by semidry blotting at $16 \mathrm{~V}$ for $1 \mathrm{~h}$, and membranes were blocked by incubation for $1 \mathrm{~h}$ a RT in $3 \%$ BSA in PBST (PBS supplemented with $0.02 \%$ Tween 20 ). Next, membranes were incubated overnigh secondary antibody in $3 \%$ BSA-PBST for $1 \mathrm{~h}$ at RT, and washed three times with PBST. Membranes were scanned using an Odyssey Infrared Imaging system (LI-COR Biosciences), and blots were acquired at 680 and $800 \mathrm{~nm}$.

\section{Image analysis and quantification}

ntification of transport in the engineered motor assay, confocal images of neuron

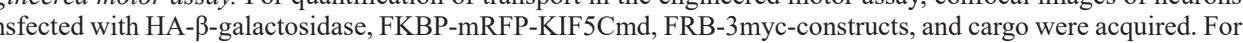
each cell, fluorescence intensity of the cargo was measured in five growth cones and the cell soma (regions of interest [ROIs] determined from $\beta$-galactosidase staining). The ratio of fluorescence intensity in a growth cone to that of the cell soma was calculated and normalized to the ratio of the condition without rapalog.

Vesicle colocalization. To investigate cargo colocalization or KIF1Atd-cargo colocalization, confoca a each cell, a line of 20-30 $\mu \mathrm{m}$ length was drawn in the axon (identified from TRIM46 staining) and two dendrites. For cargo colocalization, first the total amount of puncta of one cargo along a line was counted and then the amount of For KIF1Atd-cargo colocalization, the total amount of KIF1Atd puncta along a line was counted. Next, the amount
Folizing of colocalizing cargo particles was counted, and the percentage of colocalization was calculated.

Polarity indices. For calculation of the polarity index, neurons expessing HA-p-galctosidase and different KIF1A constructs were imaged on the upright fluorescent microscope. A line of 5- $\mu$ m width and $40-90$ m length was drawn in the axon (identifed from TRIM46 staining) and in three dendrites (ROIs determined fron $\beta$-galactosidase staining). The same ROIs were positioned in a near area where no neurons were present, and this background intensity was subtracted from raw measurements. The intensity in the axon, Ia, and the average intensity in three dendrites, Id, were then used to calculate the polarity index with the formula PI $=(\mathrm{Ia}-\mathrm{Id}) /(\mathrm{Ia}+\mathrm{Id})$.

Rab3 accumulation. For calculation of Rab3-accumulating cells, neurons expressing Rab3-GFP, shKIF1A, and indicated rescue constructs were visualized on the upright fluorescent microscope. For each condition, $\leq 100$ neurons were counted and classified as Rab3 accumulating or not Rab3 accumulating, which enabled quantificatio of the percentage of Rab3-accumulating cells.

Quantification of fluorescence intensity ratio in distal to proximal axons. To quantify axonal fluorescent Rab3 intensity, confocal images of neurons expressing indicated shRNAs and rescue constructs, stained for Rab3 from TRIM46 staining), and the same ROIs were positioned in a near area where no neurons were present. The same was done in a distal part of the proximal axon. Background intensities were subtracted from raw measurements, and the ratio of fluorescence intensity in distal to proximal axons was calculated.

Quantification of fluorescence intensity in cell soma. To quantify the fluorescence intensity in the cell soma, confocal images of neurons expressing HA- $\beta$-galactosidase and the indicated shRNAs and stained for the protein of interest were acquired. An ROI was drawn around the soma of a transfected cell (determined from $\beta$-galactosidase staining) and then positioned in a near area where no neurons were present. The same was done for two surrounding untransfected cells. Background intensities were subtracted from raw measurements, and the ratio of fluorescence ins

Eclipse Ti-E confocal microscope equipped with a perfect focus system (Nikon), a CSU-X1-A1 Spinning Disc uni (Yokogawa) and a Photometrics Evolve 512 electron-multiplying charge-coupled device camera (Roper Scientific) with a Plan Apo VC $100 \times$ NA 1.40 oil objective. For imaging, coverslips were mounted in a Ludin chamber (Life C image acquisition. 
Imaging of axonal vesicle entry in the engineered motor assay. Neurons expressing FKBP-mRFPKIF5Cmd, FRB-3myc-KIF1Atd, and GFP-tagged markers were incubated for 30 min with NF-CF555 before imaging. Neurons were then visualized in the axon (identified by NF-CF555 staining), and videos in the GFP channel were acquired as stream acquisitions at 10 frames/s for $20 \mathrm{~s}$. Videos were taken before addition of rapalog

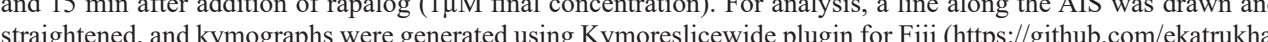
KymoResliceWide). An area of $10 \mu \mathrm{M}$ on the kymograph was selected, and all anterograde events were traced and counted for quantification of axonal vesicle entry.

Imaging of cargo motility. Neurons expressing indicated shRNAs, rescue constructs, and GFP-tagged markers were incubated for $30 \mathrm{~min}$ with NF-CF555 before imaging. Neurons were then visualized in the axon (identified by NF-CF555), and videos were acquired in the GFP channel as stream acquisitions at 10 frames/s for 20 s. For analysis, a line along the AIS was drawn, and kymographs were generated. An area of $10 \mu \mathrm{m}$ on the kymograph was selected, and all static, anterograde, and retrograde events were traced and counted for quantification

Imaging of comovement of motor and cargo. Neurons expressing the indicated GFP-tagged KIF1A constructs and NPY-RFP were visualized, and axons were determined based on morphology. Videos in the dista axon were acquired in the GFP and RFP channels as stream acquisitions at 10 frames $/ \mathrm{s}$ for $20 \mathrm{~s}$. Videos were $5 \mathrm{~s}$ were made. In these stills, the total amount of KIF1A puncta and colocalizing NPY particles were counted in the imaged axon, and the percentage of colocalization was calculated

Statistical analysis. Statistical analyses were performed using GraphPad 5. Data distribution was tested for normality using a D'Agostino and Pearson omnibus normality test. Normally distributed datasets were compare using unpaired $t$ tests. Datasets that did not have a normal distribution were compared using Mann-Whitney $U$ tests. Tests were two-tailed. Statistical tests used for each experiment are detailed in the figure legends. All error bars are displayed as mean $\pm \mathrm{SEM}$. The following $\mathrm{P}$ value conventions are used throughout the paper: $\mathrm{ns}, \mathrm{P}>0.05 ;{ }^{*}, \mathrm{P}<0.05$; **, $\mathrm{P}<0.01$; and ***, $\mathrm{P}<0.001$

\section{REFERENCES}

. (2016). The cellular mechanisms that maintain neuronal polarity. Nat. Rev. Neurosci. van den Berg, R., and Hoogenraad, C.C. (2012). Molecular motors in cargo trafficking and synapse assembly. In p. 173-196.

Van Beuningen, S.F.B., Will, L., Harterink, M., Chazeau, A., Van Battum, E.Y., Frias, C.P., Franker, M.A.M. driving the formation of parallel microtubule arrays. Neuron 88, 1208-1226.

Brummelkamp, T.R., Bernards, R., and Agami, R. (2002). Stable suppression of tumorigenicity by virus-mediate

Burack, M.A., Silverman, M.A., and Banker, G. (2000). The role of selective transport in neuronal protein sorting

Chen, J.W., Murphy, T.L., Willingham, M.C., Pastan, I., and August, J.T. (1985). Identification of two lysosomal . (2019). Disease-associated mutations hyperactivate KIF1A motility and anterograde axonal transport of synaptic vesicle precursors. PNAS 116, 18429-18434.

Coy, D.L., Hancock, W.O., Wagenbach, M., and Howard, J. (1999). Kinesin's tail domain is an inhibitory regulator of the motor domain. Nat. Cell Biol. 1, 288-292.

Farias, G.G. Britt, D.J., and Bonifacino, J.S. (2016). Imaging the polarized sorting of proteins from the golgi
complex in living neurons. Methods Mol. Biol. 1496, 13-30.

Farías, G.G., Guardia, C.M., De Pace, R., Britt, D.J., and Bonifacino, J.S. (2017). BORC/kinesin-1 ensemble drives

Fischer, G., Mignery, G.A., Baumert, M., Perin, M.S., Hanson, T.J., Burger, P.M., Jahn, R., and Sudhof, T.C. (1990)

Franker, M.A.M., and Hoogenraad, C.C. (2013). Microtubule-based transport - basic mechanisms, traffic rules and

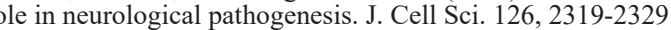

Fu, M.M., and Holzbaur, E.L.F. (2013). JIP1 regulates the directionality of APP axonal transport by coordinating
kinesin and dynein motors. J. Cell Biol. 202, 495-508. .

Gabrych, D.R., Lau, V.Z., Niwa, S., and Silverman, M.A. (2019). Going too far is the same as falling short: kinesin-3 family menbers in herediary spastic paraplegia. Fon. Cell. Neurosci. 13, 1-24.

Guardia, C.M., Farías, G.G., Jia, R., Pu, J., and Bonifacino, J.S. (2016). BORC functions upstream of kinesins Hall, D.H., and Hedgecock, E.M. (1991). Kinesin-related gene unc-104 is required for axonal transport of synaptic
vesicles in C. elegans. Cell $65,837-847$.
Hammond, J.W., Cai, D., Blasius, T.L., Li, Z., Jiang, Y., Jih, G.T., Meyhofer, E., and Verhey, K.J. (2009). Mammalian
Kinesin-3 motors are dimeric in vivo and move by processive motility upon release of autoinhibition. PLoS Biol. Kinesin-3 mo
$7,0650-0663$

Hirokawa, N., Niwa, S., and Tanaka, Y. (2010). Molecular motors in neurons: Transport mechanisms and roles in

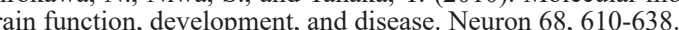

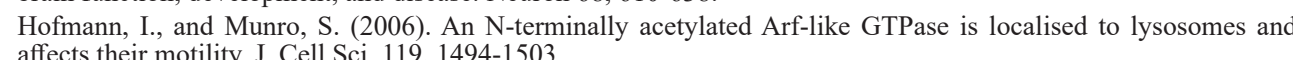
Hoogenraad, C.C. Wulf, P Schiefermeier N Stepanova, T, Galjart, N Small, J.V., Grosveld, F, De Zeeuw, C.I., and Akhmanova, A. (2003). Bicaudal D induces selective dynein-mediated microtubule minus end-directed Cansport. EMBO J. 22, 6004-6015.

Hoogenraad, C.C., Milstein, A.D., Ethell, I.M., Henkemeyer, M., and Sheng, M. (2005). GRIP1 controls dendrite ing EphB receptor trafficking. Nat. Neurosci. 8, 906-915.

Huang, C.F., and Banker, G. (2012). The translocation selectivity of the kinesins that mediate neuronal organelle

Huo, L., Yue, Y., Ren, J., Yu, J., Liu, J., Yu, Y., Ye, F, Xu, T., Zhang, M., and Feng, W. (2012). The CC1-FHA tandem
as a central hub for controlling the dimerization and activation of kinesin-3 KIF1A. Structure 20, 1550-1561 Ichinose, S., Ogawa, T., and Hirokawa, N. (2015). Mechanism of Activity-Dependent Cargo Loading via the
Phosphorylation of KIF3A by PKA and CaMKIIa. Neuron 87, 1022-1035.

Jacobson, C., Schnapp, B., and Banker, G.A. (2006). A change in the selective translocation of the kinesin-1 motor domain marks the initial specification of the axon. Neuron 49, 797-804.

Jaworski, J., Kapitein, L.C., Gouveia, S.M., Dortland, B.R., Wulf, P.S., Grigoriev, I., Camera, P., Spangler, S.A

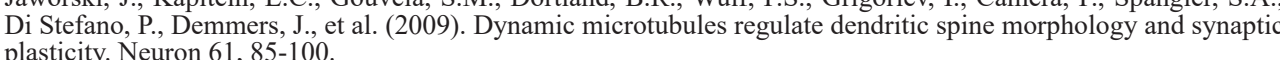

Kapitein, L.C., Schlager, M.A., Kuijpers, M., Wulf, P.S., van Spronsen, M., MacKintosh, F.C., and Hoogenraad,
C.C. (2010a). Mixed microtubules steer dynein-driven cargo transport into dendrites. Curr. Biol. 20, 290-299 Kapitein L C. Yau, K. . and Hoogenrad, C.C. (2010b). Microtubule dynamics in dendritic spines (Elsevier Inc.). Kapitein, L.C., Schlager, M.A., Van Der Zwan, W.A., Wulf, P.S., Keijzer, N., and Hoogenraad, C.C. (2010c).
Probing intracellular motor protein activity using an inducible cargo trafficking assay. Biophys. J. 99, 2143-2152. Kevenaar, J.T., Bianchi, S., Van Spronsen, M., Olieric, N., Lipka, J., Frias, C.P., Mikhaylova, M., Harterink, M., Keijzer, N., Wulf, P.S., et al. (2016). Kinesin-binding protein controls microtubule dynamics and cargo trafficking by regulating kinesin motor activity. Curr. Biol. 26, 849-861.

Klassen, M.P., Wu, Y.E., Maeder, C.I., Nakae, I., Cueva, J.G., Lehrman, E.K., Tada, M., Gengyo-Ando, K., Wang, G.J., Goodman, M., et al. (2010). An Arf-like small G protein, ARL-8, promotes the axonal transport of presynaptic

Klopfenstein, D.R., and Vale, R.D. (2004). The lipid binding pleckstrin homology domain in UNC-104 kinesin is
necessary for synaptic vesicle transport in Caenorhabditis elegans. Mol Biol Cell 15, 3729-3739. Klopfenstein, D.R., Tomishige, M., Stuurman, N., and Vale, R.D. (2002). Role of phosphatidylinositol(4,5) bisphosphate organization in membrane transport by the Unc104 kinesin motor. Cell 109, $347-358$

Kwinter, D.M., Lo, K., Mafi, P., and Silverman, M.A. (2009). Dynactin regulates bidirectional transport of densecore vesicles in the axon and dendrites of cultured hippocampal neurons. Neuroscience 162, 1001-1010. Lee, J.R., Shin, H., Choi, J., Ko, J., Kim, S., Lee, H.W., Kim, K., Rho, S.H., Lee, J.H., Song, H.E., et al. (2004). A intramolecular interaction between the FHA domain and a coiled coil negatively regulates the kinesin motor KIF $1 \mathrm{~A}$.

Lo, K.Y., Kuzmin, A., Unger, S.M., Petersen, J.D., and Silverman, M.A. (2011). KIF1A is the primary anterograde motor protein requi

Millecamps, S., and Julien, J.P. (2013). Axonal transport deficits and neurodegenerative diseases. Nat. Rev. Neurosci.

Miller, K.E., DeProto, J., Kaufmann, N., Patel, B.N., Duckworth, A., and Van Vactor, D. (2005). Direct observation

Miyoshi, J., and Takai, Y. (2004). Dual role of DENN/MADD (Rab3GEP) in neurotransmission and neuroprotection.

Niwa, S., Tanaka, Y., and Hirokawa, N. (2008). KIF1Bß- and KIF1A-mediated axonal transport of presynaptic Niwa, S., Lipton, D.M., Morikawa, M., Zhao, C., Hirokawa, N., Lu, H., and Shen, K. (2016). Autoinhibition of a neuronal kinesin UNC-104/KIF1A regulates the size and density of synapses. Cell Rep. 16, 2129-2141.

O'Shea, E., Klemm, J., Kim, P., and Alber, T. (1991). X-ray structure of the GCN4 leucine zipper, a two-stranded, Okada Y Y Yail. Science (80-.). 245, 539-544. Hirokawa, N. (1995). The neuron-specific kinesin superfamily protein KIF1A is a unique monomeric motor for anterograde axonal transport of synaptic vesicle precursors. Ce Ren, J., Wang, S., Chen, H., Wang, W., Huo, L., and Feng, W. (2018). Coiled-coil 1-mediated fastening of the neck
and motor domains for kinesin-3 autoinhibition. PNAS 115, E11933-E11942. 
Rivire, J.B., Ramalingam, S., Lavastre, V., Shekarabi, M., Holbert, S., Lafontaine, J., Srour, M., Merner, N.,
Rochefort, D., Hince, P., et al. (2011). KIF1A, an axonal transporter of synaptic vesicles, is mutated in hereditary Rochefort, D., Hince, P., et al. (2011). KIF1A, an axonal transporter of synaptic vesicles, is mutated in hereditary

Rosa-Ferreira, C., and Munro, S. (2011). Arl8 and SKIP act together to link lysosomes to kinesin-1. Dev. Cell 21,

Schlager, M.A., Kapitein, L.C., Grigoriev, I., Burzynski, G.M., Wulf, P.S., Keijzer, N., De Graaff, E., Fukuda, M., Shepherd, I.T., Akhmanova, A., et al. (2010). Pericentrosomal targeting of Rab6
related protein 1 (BICDR-1) regulates neuritogenesis. EMBO J. 29, 1637-1651.

Shin, H., Wyszynski, M., Huh, K.H., Valtschanoff, J.G., Lee, J.R., Ko, J., Streuli, M. Weinerg, R J., Sheng M., and Kim, E. (2003). Association of the kinesin motor KIF 1A with the multimodular protein liprin- $\alpha$. J., Biol. Chem.

Shishido, H., and Maruta, S. (2012). Engineering of a novel $\mathrm{Ca}^{2+}$-regulated kinesin molecular motor using a

Siddiqui, N., Zwetsloot, A.J., Bachmann, A., Roth, D., Hussain, H., Brandt, J., Kaverina, I., and Straube, A. (2019). Soppina, V.. Norris S S R Dizail A autoinhibition and activate intracellular transport. Nat. Commun. 10, 1-16. of mammalian kinesin-3 motors results in superprocessive motion. PNAS 111, $5562-5567$.

Stucchi, R., Plucińska, G., Hummel, J.J.A., Zahavi, E.E., Guerra San Juan, I., Klykov, O., Scheltema, R.A., Altelaa DCV binding and Lipun

Tanaka, M., Miyoshi, J., Ishizaki, H., Togawa, A., Ohnishi, K., Endo, K., Matsubara, K., Mizoguchi, A., Nagano, T., Sato, M., et al. (2001). Role of Rab3 GDP/GTP exchange protein in synaptic vesicle trafficking at the mouse an der Vaart, B, van. Riel, W.E., Ceodhi, H. Kevenar, J.T., Katrukha, E.A., Gumy, L., Bouchet, B.P., Grigoriev, 1., Spangler, S.A., Yu, K. Lou, et al. (2013). CFEOM1-associated kinesin KIF21A is a cortical microtubule growth

Verhey, K.J., and Hammond, J.W. (2009). Traffic control: Regulation of kinesin motors. Nat. Rev. Mol. Cell Biol. van Vlijmen, T., Vleugel, M., Evers, M., Mohammed, S., Wulf, P.S., Heck, A.J.R., Hoogenraad, C.C., and van der Sluijs, P. (2008). A unique residue in rab3c determines the interaction with novel binding protein Zwint-1. FEBS Lett. 582, 2838-2842. Wang, X., and Schwarz, T.L.

de Wit, J., Toonen, R.F., Verhaagen, J., and Verhage, M. (2006). Vesicular trafficking of semaphorin 3A is activitydependent and dincers between axons and dendrites. Traffic $7,1060-1077$.

Wu, Y.E., Huo, L., Maeder, C.I., Feng, W., and Shen, K. (2013). The balance between capture and dissociation of

Xue, X., Jaulin, F., Espenel, C., and Kreitzer, G. (2010). PH-domain-dependent selective transport of p75 by

Zahn, T.R., Angleson, J.K., MacMorris, M.A., Domke, E., Hutton, J.F., Schwartz, C., and Hutton, J.C. (2004). Dense in Caenorhabditis elegans neurons and the role of kinesin UNC-104. Traffic 5, 544-559. homolog unc-104 is important for site-specific synapse maturation. Front. Cell. Neurosci. 10, 1-14.

\section{SUPPLEMENTAL INFORMATION}
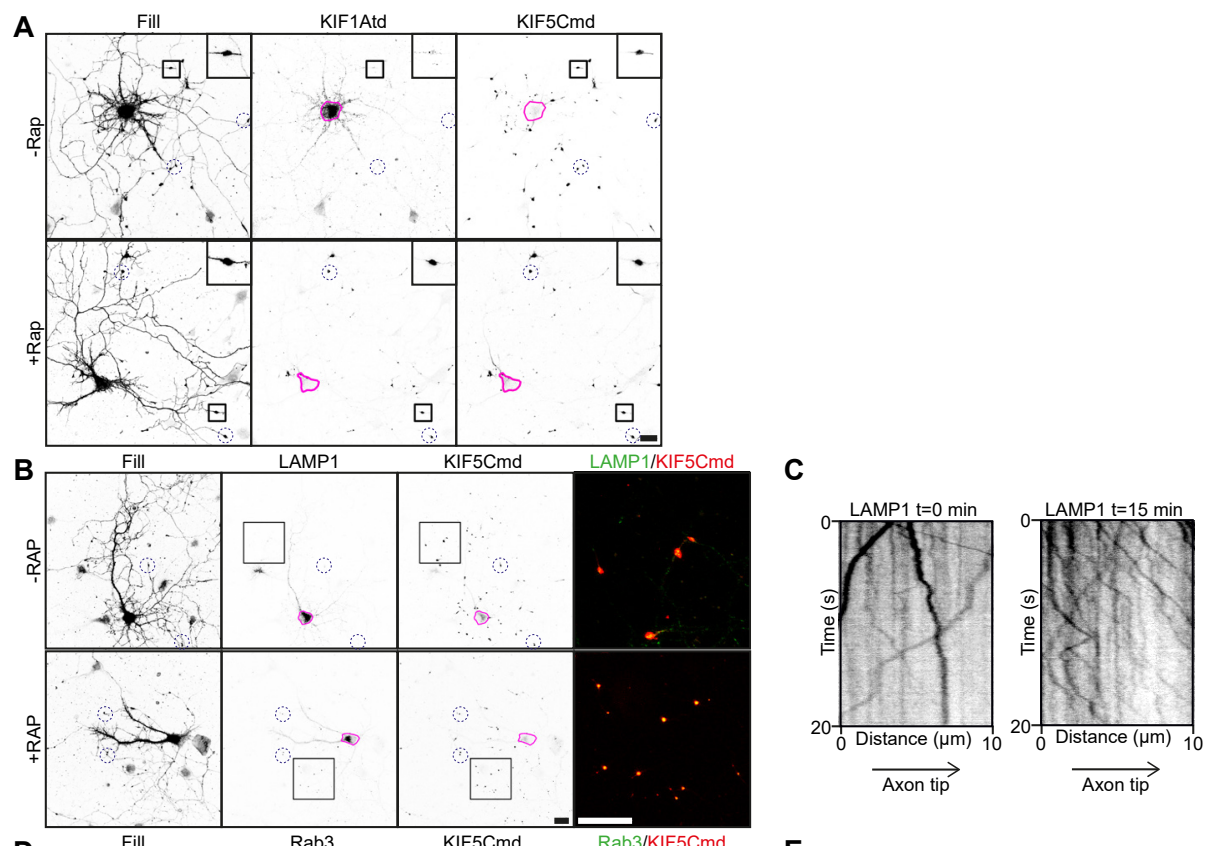

E
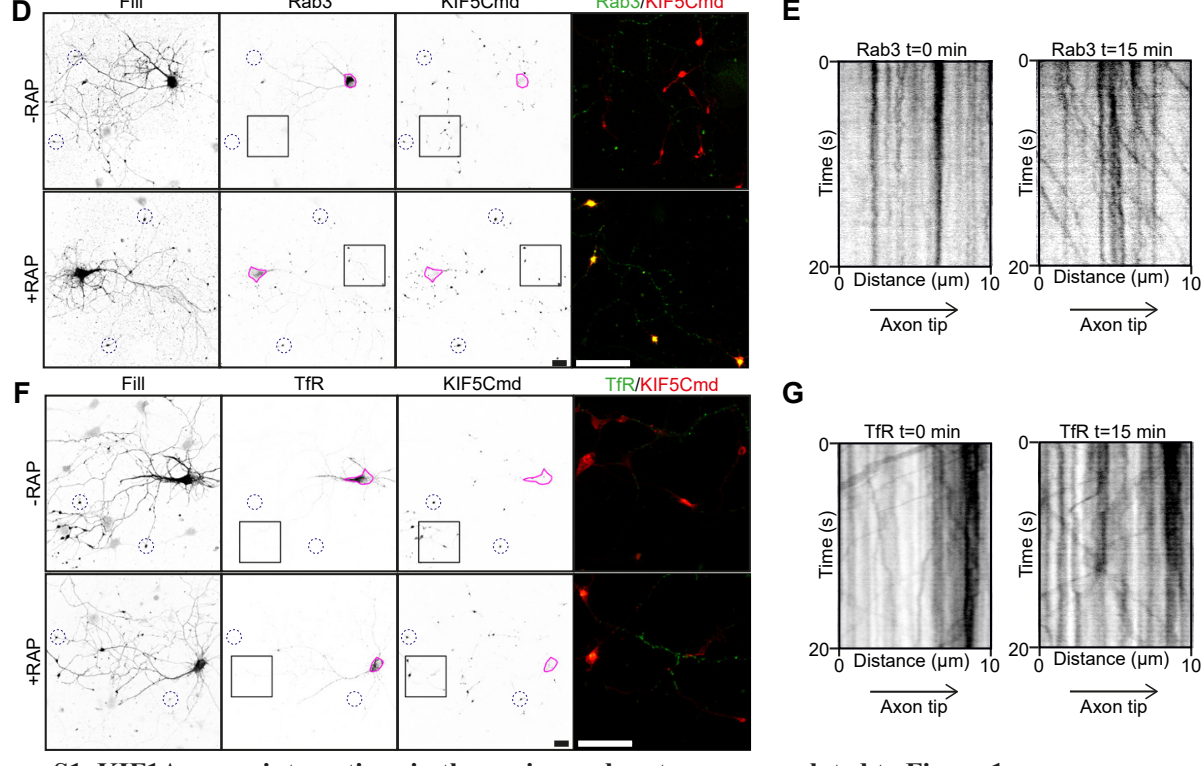

G

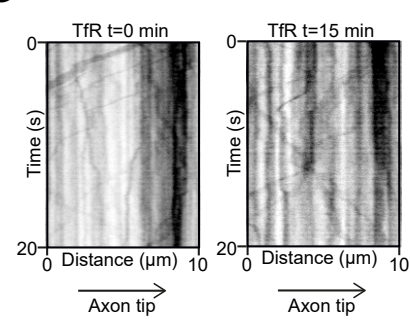

ssay - related to Figure 1

Images validating the engineered motor assay and showing lysosome, SV, and endosomal vesicle localization and motility in the assay. (A) Representative images of hippocampal neurons coexpressing FRB-3myc-KIFlAtd an the cell soma. Blue dotted circles indicate examples of axonal tips. Zooms of the boxed regions are shown in the top right of each image (B,D,F) Representative images of hippocampal neurons coexpressing FRB-3myc-KIF1 Atd FKBP-mRFP-KIF5Cmd, and LAMP1-GFP (B), Rab3-GFP (D), or TfR-GFP (F) without (upper panels) or with (lower panels) addition of $1 \mu \mathrm{M}$ rapalog. Pink lines mark the cell soma. Blue dotted circles indicate examples of axonal tips. Zooms of the boxed regions are shown as a merge on the right. $(\mathbf{C}, \mathbf{E}, \mathbf{G})$ Representative kymographs showing movement of LAMP1 (C), Rab3 (E), or TfR (G) vesicles in the AIS before (left) and 15 min after (right) addition of $1 \mu \mathrm{M}$ rapalog. Scale bars: $20 \mu \mathrm{m}$ 


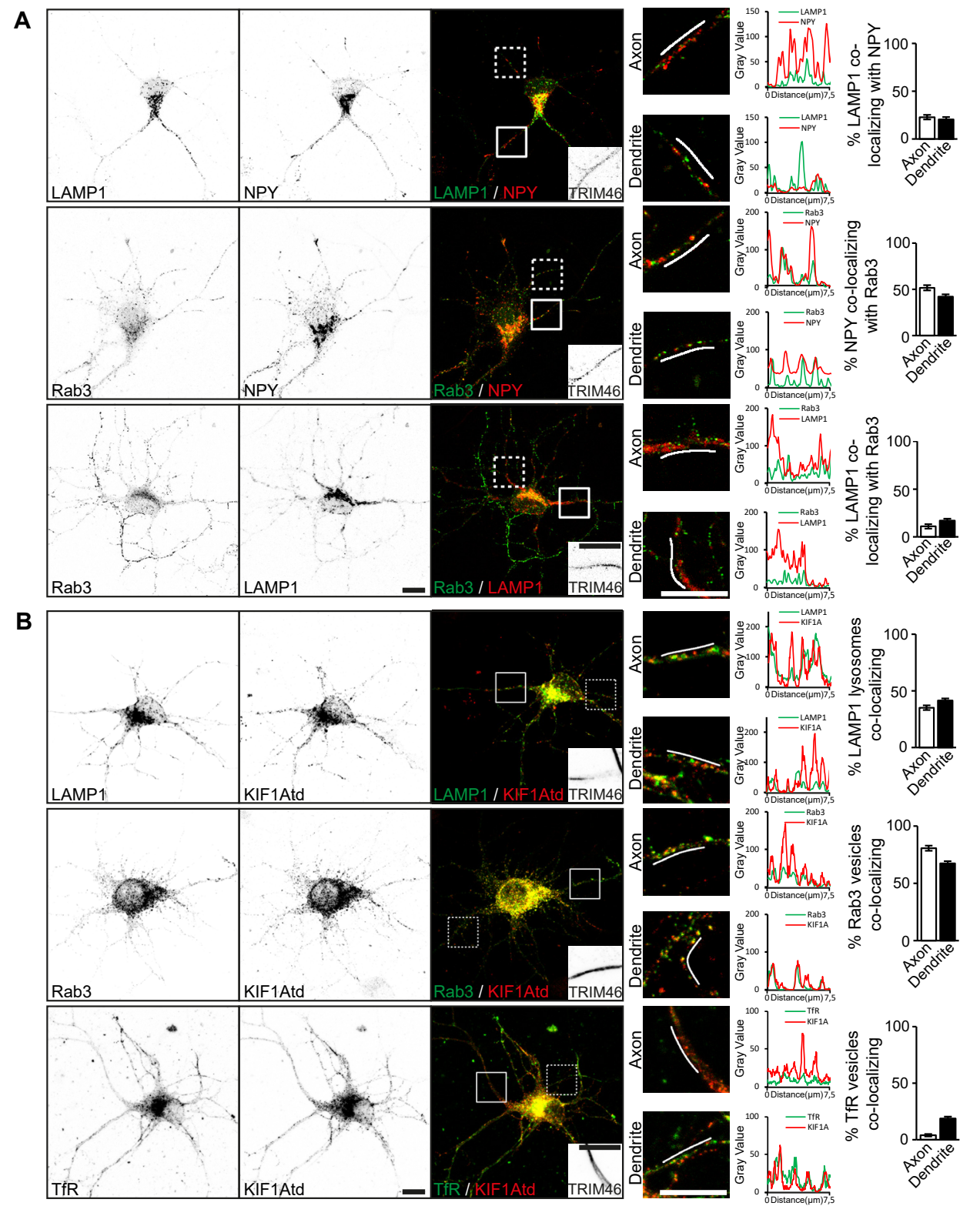

Figure S2. Colocalization of cargo vesicles and KIF1A - related to Figure 1

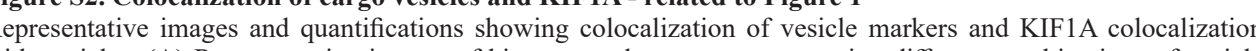
warkers. White boxes on the merged image mark the axon (continuous line) and a dendrite (dinations of vesicle taining in the axon is shown in the merged image. Merged zooms of the boxes are depicted on the right Graph show the intensity of cargo along the line marked in the zooms. Quantifications of the percentage of vesicle markers colocalizing with each other in the axon and dendrites are shown on the right $(\mathrm{N}=3, \mathrm{n}=29-33$ axons and 87-99 dendrites). (B) Representative images of hippocampal neurons coexpressing KIF1Atd and GFP-tagged LAMP1, Rab3, or TfR. White boxes on the merged image mark the axon (continuous line) and a dendrite (dotted line). TRIM46 staining in the axon is shown in the merged image. Merged zooms of the boxes are depicted on the right. Graphs show the cargo (green) and KIFIA (red) intensity along the line marked in the zooms. Quantifications of the percentage of vesicle markers colocalizing with KIFIA in the axon and dendrites are shown on the right $(\mathrm{N}=3$,

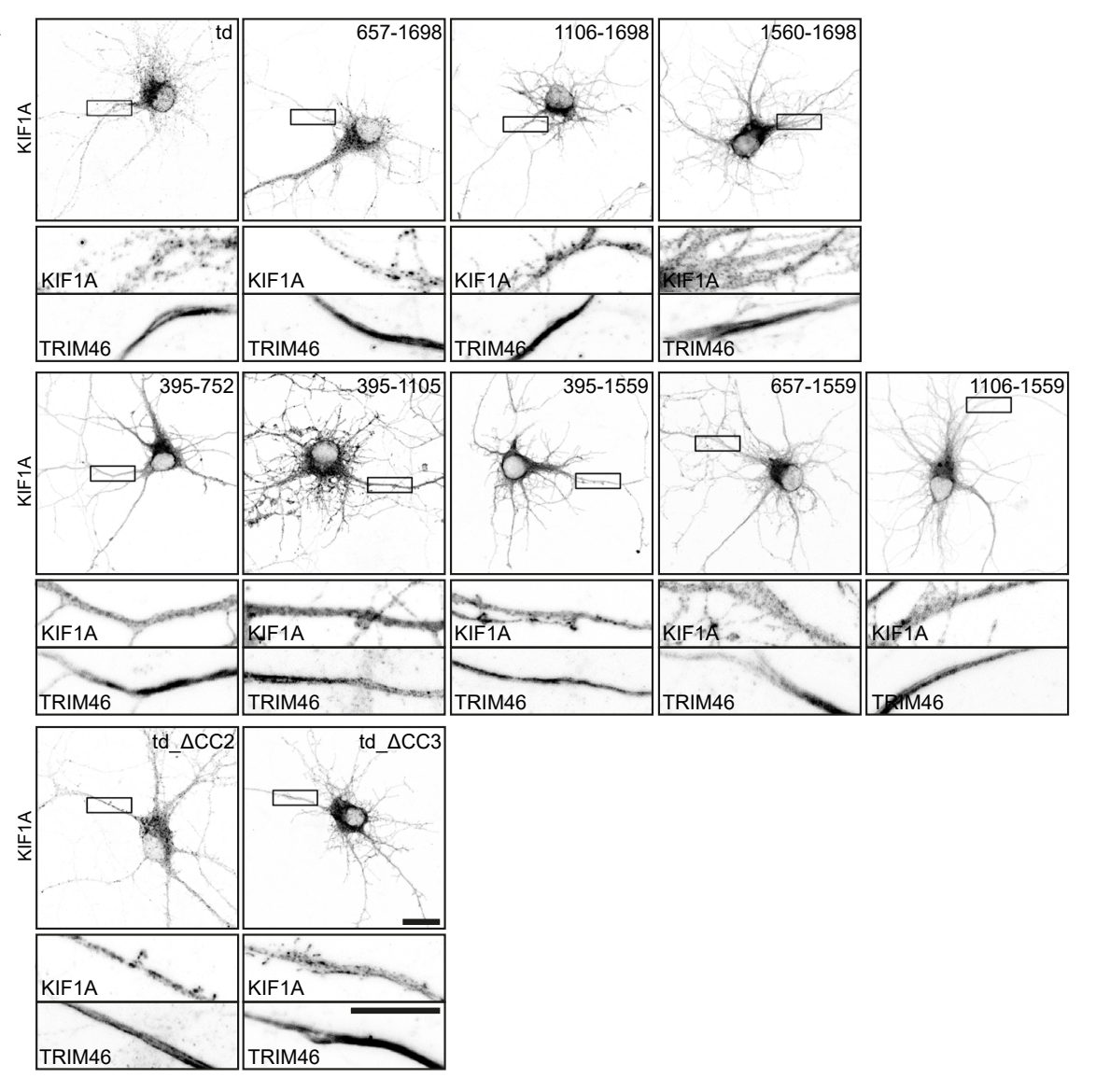

Figure S3. Expression of KIF1Atd truncation constructs in hippocampal neurons - related to Figure 2 (A) Representative images of hippocampal neurons expressing KIF1A deletion constructs (shown in Fig 2B,F,J) below. Scale bars: 20 um (upper images), $10 \mu \mathrm{m}$ (lower zooms). 
A
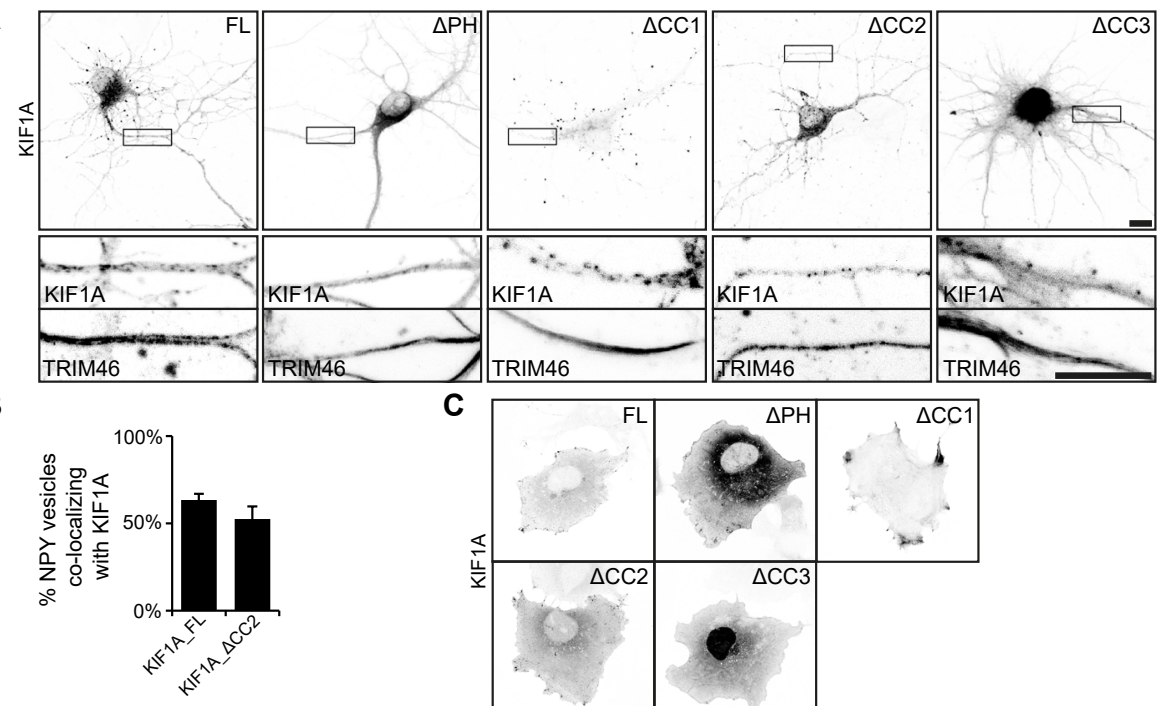

D
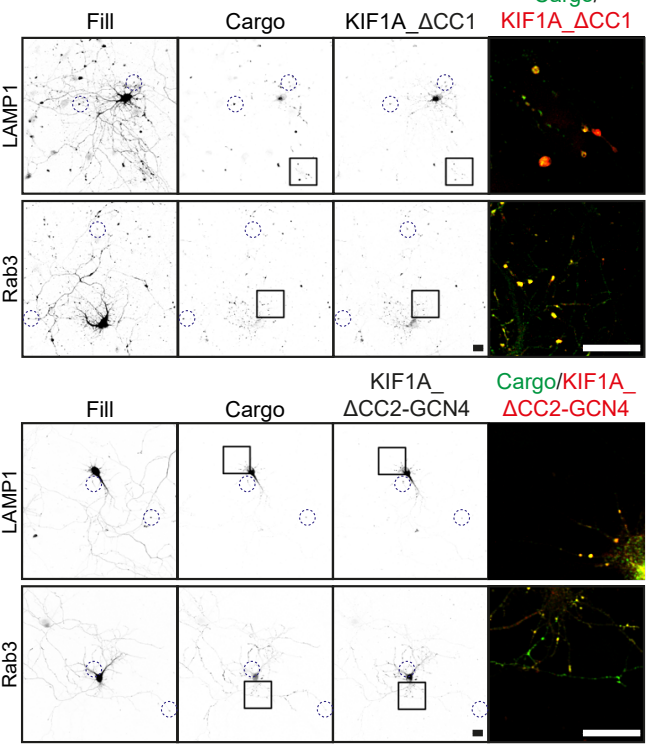

$\mathbf{F}$

E

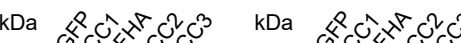

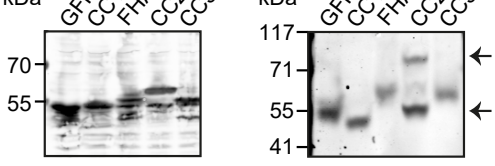

$\Delta \mathrm{CCC}-\mathrm{GCN}$

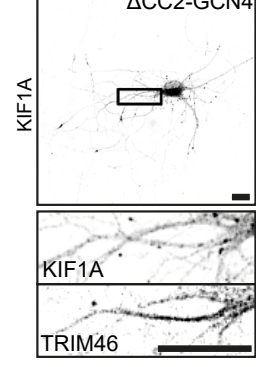

gure S4. Expression of KIFIA constructs and colocalization with cargo - related to Figure 3 and 4 Example images of cells expressing KIFIA constructs in neurons and in COS7 cells or together with cargo in neurons and dimerization analysis on Westen blot. (A) Representative inages of hippocantpal neurons expressing KIF1A deletion constructs (shown in Fig 3B) including zooms of the axon, marked by the boxes. TRIM46 staining and the KIFIA channel in the axon are shown below. (B) Quantification of the percentage of NPY vesicles $\triangle C$ C2). (C) Representative images of COS7 cells expressing different KIF1A constructs. (D) Representative im of hippocampal neurons coexpressing KIF1A_ACC1 and LAMP1 or Rab3. Blue dotted circles indicate examples of distal tips. Merged image of the boxed region is shown on the right. (E.F) Normal (E) and native (F) SDSPAGE analysis of GFP-fused KIF1A domains expressed in HEK293T cell lysates. Arrows (F) mark the bands of monomeric and dimeric CC2 domain. (G) Representative images of hippocampal neurons coexpressing KIF1A $\triangle \mathrm{CC} 2$-GCN4 and LAMP1 or Rab3. Blue dotted circles indicate examples of distal tips. Merged image of the boxed region is shown on the right. (H) Representative image of a hippocampal neuron expressing KIFIA_ $\triangle \mathrm{CC} 2-\mathrm{GCN}$ (shown in Fig 4H) including zoom of the axon, marked by the box. TRIM46 staining and the KIF1A channel in the axon are shown below. Scale bars: $10 \mu \mathrm{m}(\mathrm{A}, \mathrm{C}, \mathrm{D}, \mathrm{G}), 20 \mu \mathrm{m}(\mathrm{H})$

\section{A $\overline{\overline{\bar{c}}}$

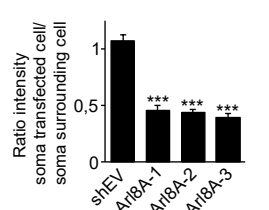

C
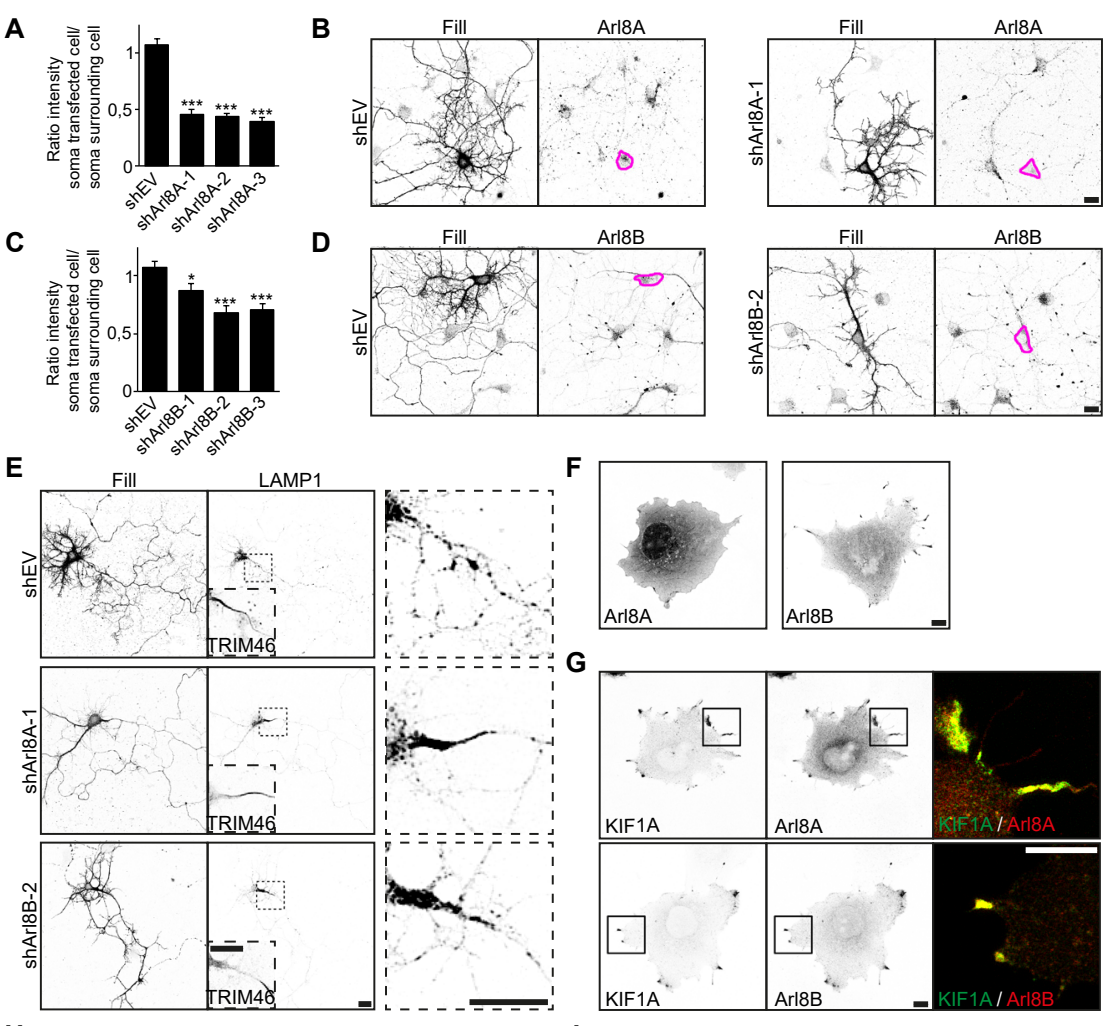

H.
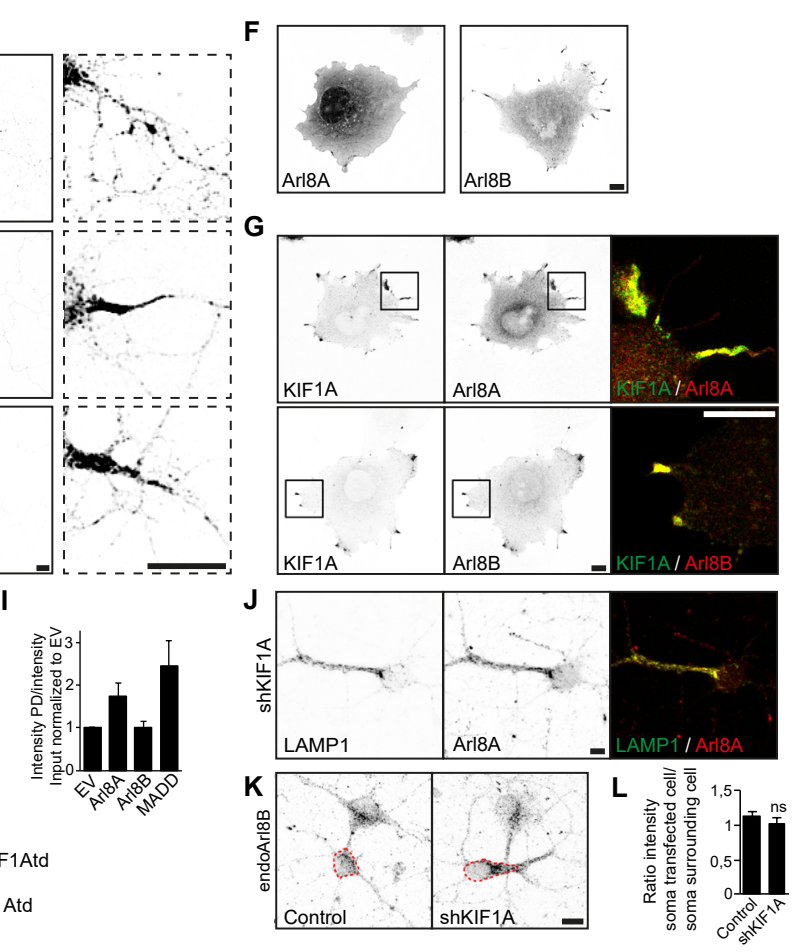

Figure S5. Identification of Arl8A and MADD as specific KIF1A adaptors - related to Figure 5 Validation of Arl8 shRNAs and investigation of Arl8A and MADD as specific KIF1A adaptors. (A,C) Quantification of the ratio of endogenous Arl8A (A) or Arl8B (C) intensity in a transfected cell to that of a surrounding cell in control and with different shRNAs against $\operatorname{Arl} 8 \mathrm{~A}$ ( $\mathrm{A}$ ) or $\operatorname{Arl} 8 \mathrm{~B}(\mathrm{C} ; \mathrm{N}=4, \mathrm{n}=40-35)$. (B,D) Representative images of hippocampal neurons expressing shEV, shArl8A-1 (B), or shArl8B-2 (D), immunostained for Arl8A (B) or Arl8B (D). Pink lines mark the cell soma of the transfected cell. (E) Representative images of hippocampal neuron coexpressing LAMF together with shEV, shArl8A-1, or shArr8B-2. TRIM46 staining in the dotted box is shown COS7 cells expressing Arl A or Arl8B. (G) Representative images of COS7 cells coexpressing KIF1A together of Arl8A or Arl8B. Merged image of the boxed region is shown on the right (H) Pull-down experiment showing the interaction between KIF1A 395-1105 and bioGFP-adaptors in HEK293T cell lysates. (I) Quantification of the ratio of KIF1A 395-1105 intensity in the pull-down fraction to that of the input fraction normalized to bioGFP-EV (N=3). (J) Representative image of a hippocampal neuron coexpressing shKIF1A and LAMP1-GFP and immunostained for Arl8A. (K) Representative images of the soma of hippocampal neurons immunostained for Arl8B in contro or when transfected with KIF1A shRNA. Dotted red lines mark the cell soma. (L) Quantification of the ratio of endogenous Arl8B intensity in a transfected cell to that of a surrounding cell in control or KIFIA KD ( $N=3, n=29$ 30). Data are displayed as means \pm SEM. Unpaired t test (A), Mann-Whitney $\mathrm{U}$ test $(\mathrm{C}, \mathrm{L}),{ }^{*}, \mathrm{P}<0.05 ;{ }^{* * *}, \mathrm{P}<0.001$ scale bars: $20 \mu \mathrm{m}$ (B,D,E), $10 \mu \mathrm{m}(\mathrm{F}, \mathrm{G}, \mathrm{J}, \mathrm{K})$ 


\section{Combined kinesin-1 and kinesin-3 activity drives axonal trafficking of TrkB receptors in Rab6 carriers}

Eitan Erez Zahavi1,4, Jessica J.A. Hummel ${ }^{1}$, Yuhao Han ${ }^{1,5}$, Citlali Bar', Riccardo Stucchi ${ }^{1,2}$, Maarten Altelaar ${ }^{2}$, and Casper C. Hoogenraad ${ }^{1,3}$

Dev. Cell (2021);56:494-508

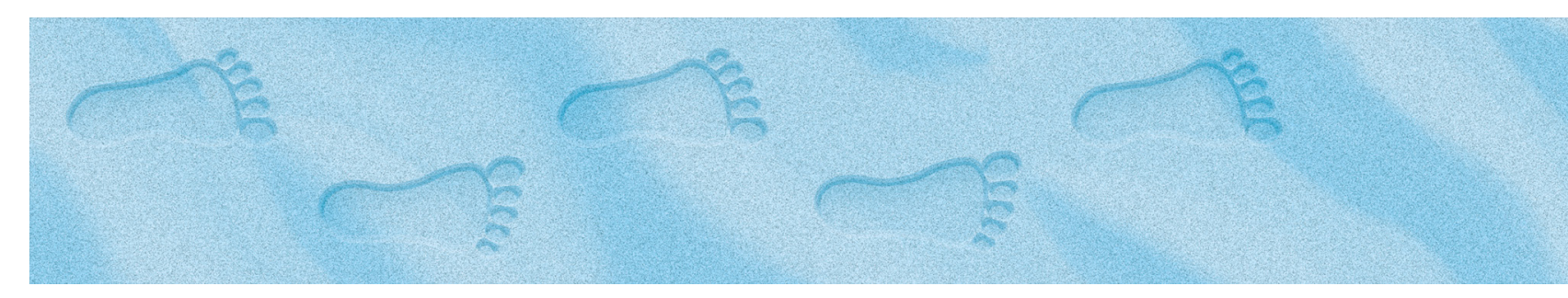

${ }^{1}$ Cell Biology, Neurobiology and Biophysics, Department of Biology, Faculty of Science, Utrecht University, 3584 CH Utrecht, the Netherlands.

${ }^{2}$ Biomolecular Mass Spectrometry and Proteomics, Bijvoet Center for Biomolecular Research and Utrecht Institute for PharmaceuticalSciences, Utrecht University, Padualaan 8, 3584 Utrecht, the Netherlands.

${ }^{3}$ Department of Neuroscience, Genentech, Inc., South San Francisco, CA 94080, USA

${ }^{4}$ Present address: Department of Biomolecular Sciences, Weizmann Institute of Science, Present address: Department of Biomolecular Sciences, Weizmann Institute of Science,
Rehovot 76100, Israel. Rehovot 76100, Israel.

${ }^{5}$ Present address: DFG Emmy Noether Group "Neuronal Protein Transport," Center for Molecular Neurobiology, ZMNH, University Medical Center Hamburg-Eppendorf, 20251 Hamburg, Germany. 


\section{SUMMARY}

Neurons depend on proper localization of neurotrophic receptors in their distal processes for their function. The Trk family of neurotrophin receptors controls neuronal survival, differentiation, and remodeling and are well known to function as retrograde signal carriers transported from the distal axon toward the cell body. However, the mechanism driving anterograde trafficking of Trk receptors into the axon is not well established. We used microfluidic compartmental devices and inducible secretion assay to systematically investigate the retrograde and anterograde trafficking routes of TrkB receptor along the axon in rat hippocampal neurons. We show that newly synthesized TrkB receptors traffic through the secretory pathway and are directly delivered into axon. We found that these TrkB carriers associate and are regulated by Rab6. Furthermore, the combined activity of kinesin-1 and kinesin- 3 is needed for the formation of axon-bound TrkB secretory carriers and their effective entry and processive anterograde transport beyond the proximal axon.

\section{INTRODUCTION}

Neurons are uniquely structured cells with an extreme degree of morphological polarity between their dendritic and axonal domains. This segregation enables the efficient neuronal integration of multiple post-synaptic inputs in the somatodendritic compartment and the transmission of processed information via the axon to the pre-synapse. The function of transport of membrane protein that comprise the pre- and post-synaptic receptor repertoire (Bentley and Banker, 2016). A particularly difficult logistic challenge for the mammalian neuron is the delivery of membrane proteins to the distal axon processes due to its sheer distance from the subcellular sites in which their biosynthesis takes place, namely that in neurons of the central nervous system the rough endoplasmic reticulum (RER) and the Golgi apparatus reside exclusively in the soma and dendritic compartment (Britt et al., 2016).

The family of tropomyosin-related kinases (Trk) receptors, including TrkA, TrkB, and $\mathrm{TrkC}$, provides a prime example of membrane proteins that undergo long-distance axonal trafficking to regulate growth and survival of developing neurons. The cognate ligand of TrkB, brain-derived neurotrophic factor (BDNF), is a predominant neurotrophin in the brain that plays a major role in regulating neuronal development, survival, and remodeling by that plays a major role in regulating neuronal development, survival, and remodeling by
signaling in the pre- and post-synapse in the peripheral and central nervous system (Bothwell, signaling in the pre- and post-synapse in the peripheral and central nervous system (Bothwell,
2016; Park and Poo, 2013). As such, the localization and activity of the TrkB receptor in the distal axon is crucial for both its local and retrograde (axon-to-soma) neurotrophic functions (Harrington and Ginty, 2013; Scott-Solomon and Kuruvilla, 2018). The cellular mechanisms underlying the retrograde delivery of the neurotrophic signal have been described by the established signaling endosome model, by which the internalized receptor-ligand complex is transported along the axon by dynein motors with its downstream signaling intact (Cosker and Segal, 2014). Nevertheless, little is understood about the molecular mechanisms responsible for the delivery Trk receptors into the axon.

Polarized trafficking of membrane proteins to the axon follows one of two main delivery routes: (1) direct path via secretory carriers that fuse with axonal membrane and (2) an indirect route, also termed the transcytosis pathway, in which these carriers are first delivered to somatodendritic membranes, followed by endocytosis re-distribution of the protein to the axon (Lasiecka and Winckler, 2011; Sampo et al., 2003; Wisco et al., 2003). Axonal delivery of TrkA, the receptor for nerve growth factor (NGF), has been shown to follow a transcytosis route from somatodendritic to cervical ganglia (SCG) neurons (Ascaño et al., 2009). The anterograde trafficking of the transcytosed TrkA cargo is regulated by a feedback mechanism induced by retrograde transport of NGF-stimulated TrkA, which activates somatic PTP1B to de-phosphorylate the receptor and re-distributes it from somatodendritic membranes to the axon (Yamashita et al., 2017). Soma-to-axon transcytosis of TrkB has not been reported; however, it is known that its sorting into Rab11 compartments regulates local recycling to the plasma membrane in dendrites and at post-synaptic domains specifically (Huang et al., 2013; Lazo et al., 2013; Song et al., 2015). Efficient anterograde delivery of axon-bound membrane proteins depends primarily on kinesin-1 and kinesin-3 families of motors (Bentley and Banker, 2016). Kinesin- 1 motors enable axon-specific transport owing to their preferential binding to acetylated and detyrosinated microtubules (MTs) that are enriched in the axon, and to the homogeneous detyrosinated microtubules (MTs) that are enriched in the axon, and to the homogeneous
orientation of MT plus ends pointing toward the axon tips, while in dendrites acetylated orientation of MT plus ends pointing toward the axon tips, while in dendrites acetylated
MTs are oriented toward the soma, hence, sequestering kinesin-1 entry (Fourriere et al., 2020). Kinesin-3 KIF1 motors, which drive both dendritic and axon transport of synaptic and dense core vesicles, have special axon delivery roles. In DRG neurons, KIF1A enables cargo to traverse the pre-axonal trafficking barrier for further transport via kinesin-1 (Gumy et al., 2017) and in localized targeting of synaptic vesicles to the pre-synapse in hippocampa neurons (Guedes-Dias et al., 2019). Anterograde axon transport of TrkB was shown to be (Arimura et al., 2009; Tanaka et al., 2016). Both studies postulated these to be secretory vesicles derived from the Golgi apparatus, which requires further examination.

Protein trafficking from the secretory pathway in neurons has focused mainly on dynamics in dendrites, highlighting the interplay between secretory organelle localization, motor proteins, and Rab proteins in regulating local trafficking for specific membrane proteins (Kennedy and Hanus, 2019). Recent studies have shown that upon exiting the secretory pathway, membrane proteins undergo selective transport or retention in order to reach the axon or dendrites in a specific manner; yet, the underlying motor and membrane sorting and trafficking proteins that regulate this selectivity remain largely unknown.

In this study, we set to elucidate the trafficking and transport mechanism that delivers TrkB to the axon in polarized hippocampal neurons. Using a combination of compartmental surface labeling and inducible secretion assays, we dissected its transport dynamics in the endosomal and secretory pathways. Our results show that delivery of TrkB into the axon is driven by direct trafficking and transport from the secretory system in Rab6 carriers and the combined activity of kinesin- 1 and kinesin-3 motors.

\section{RESULTS}

\section{Endocytosed TrkB is coupled to retrograde transport in the axon}

To probe the general transport dynamics of TrkB we performed live imaging of transfected TrkB-GFP in hippocampal neurons axon and dendrites. At steady state, TrkB carriers in the axon are transported toward the distal axon (anterogradely) as well into the som (retrogradely), while in dendrites the receptor mobility is slower and with more frequen directional switches (Fig 1A; Video S1). Both the retrograde and anterograde carriers display occasional pauses between fast and processive mobility. During their processive mobility intervals, axonal TrkB-GFP moves at a mean velocity of 0.85 and $0.61 \mu \mathrm{m} / \mathrm{s}$ in the anterograde and retrograde directions, respectively (Fig 1B). The observed bidirectional transport in the axon and the slow dynamics of dendritic transport fit with established models of Trk receptor axonal transport driven by kinesin and dynein motors (Scott-Solomon and Kuruvilla, 2018). Internalization of plasma membrane Trk receptors and subsequent endosomal sorting is coupled to receptor transport (Cosker and Segal, 2014). To understand how internalized TrkB receptors are trafficked and transported, we transfected neurons with myc-SNAP-tagged TrkB and validated its proper intracellular and surface expression using myc immunolabeling (Fig S1A). We then used the surface-SNAP tag system to follow receptor trafficking and transport in live neurons (Fig 1C). Addition of the TrkB ligand BDNF induced the internalization of surface-labeled SNAP-TrkB and its accumulation in intracellular puncta in the course of 30-60 min (Fig 1D; Fig S1B,C). Internalized Trk is tranper pized in dendrites, which is similar to the difference observed for TrkB-GFP (Fig 1A,E). 

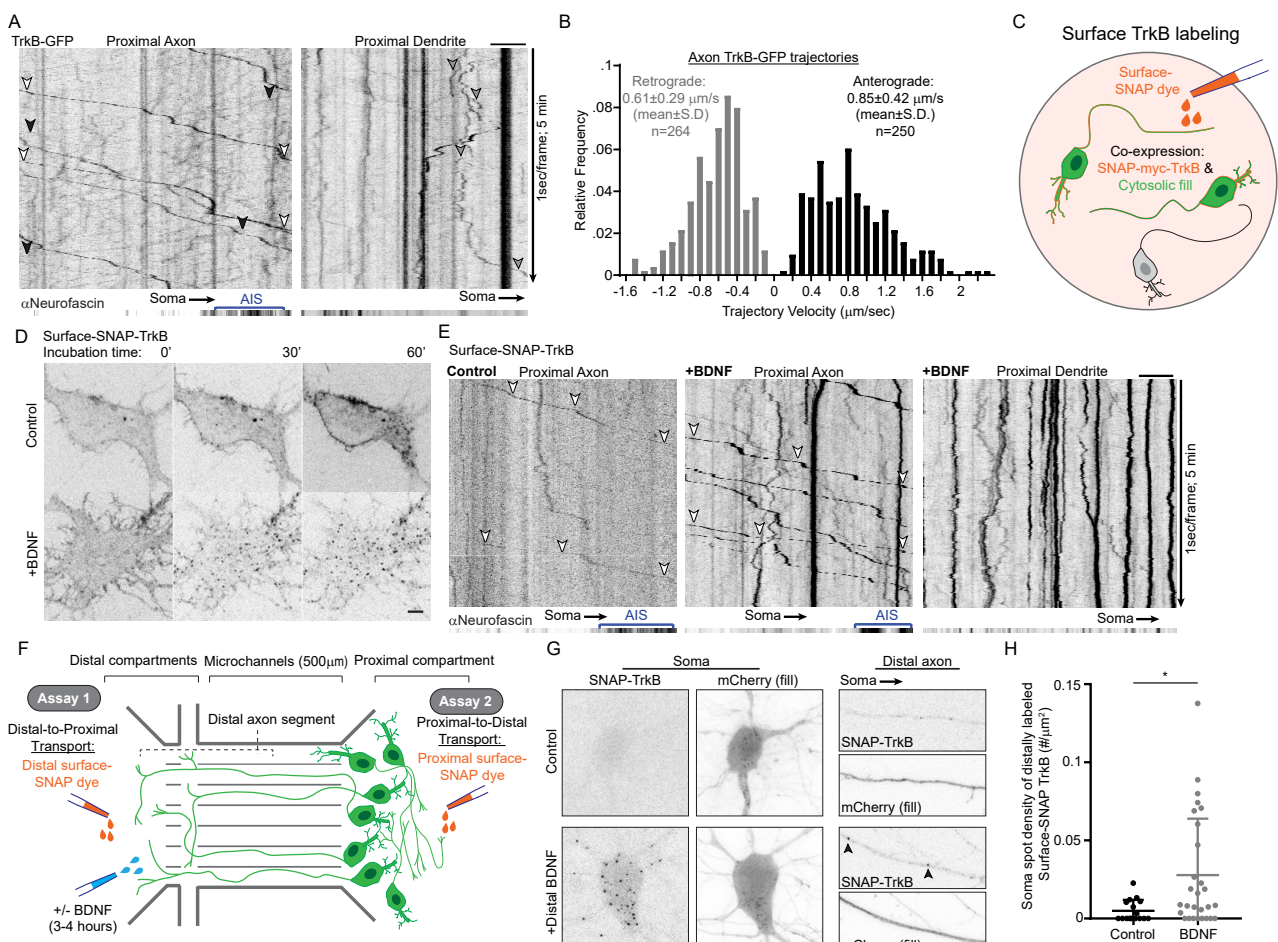

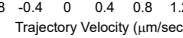
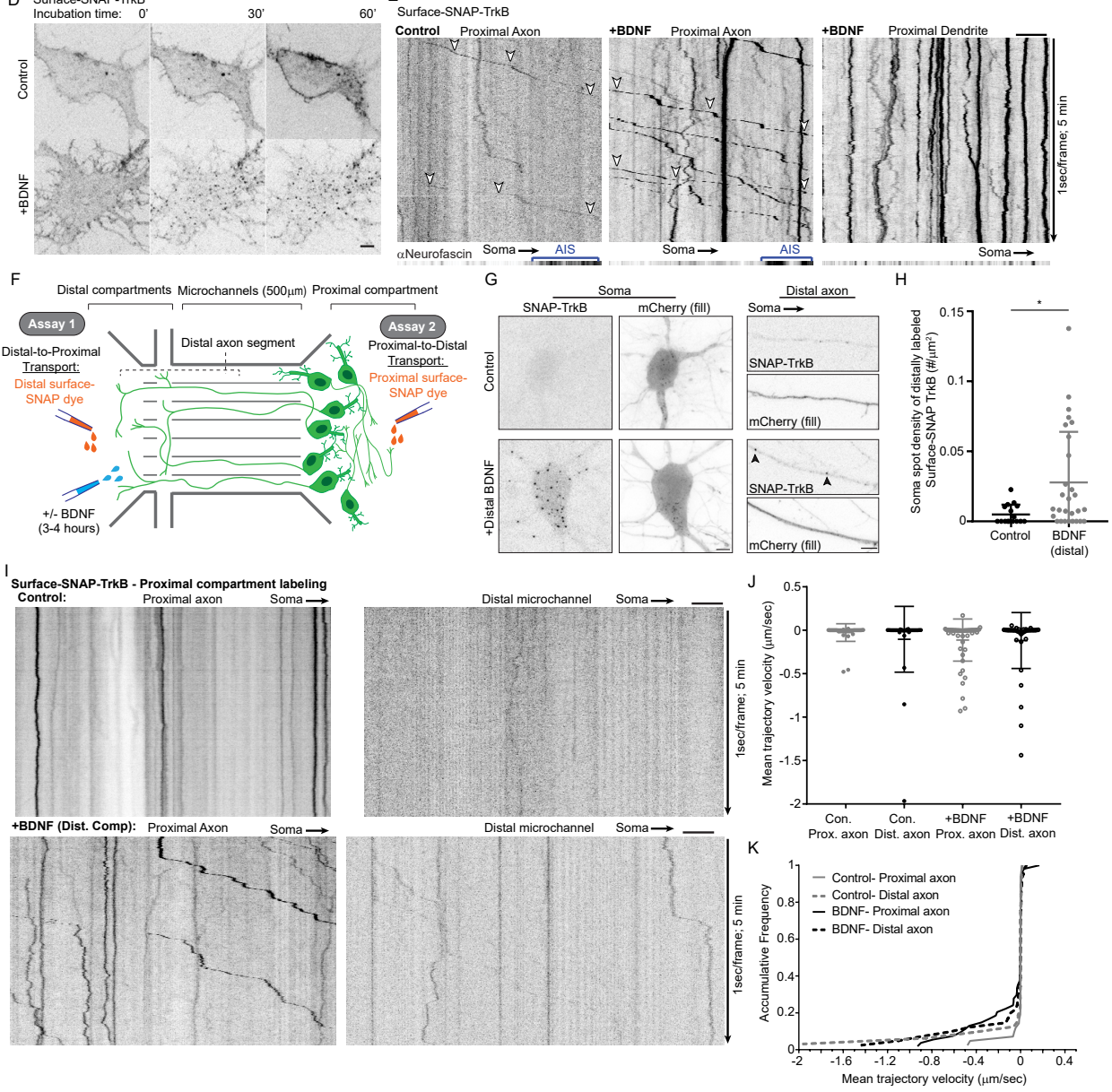

Figure 1. Axonal transport of internalized TrkB receptors is predominantly retrograde
(A) Kymographs depict bidirectional motility of TrkB-GFP in the proximal axon and dendrite. Neurofascin signal (extracellular antibody) was acquired at the first time point and is projected to localize the AIS. TrkB-GFP signal was acquired at $1 \mathrm{~s} /$ frame for $5 \mathrm{~min}$. Examples of processively moving retrograde and anterograde TrkB cargos in the axon are marked by white and black arrowheads, respectively. Example of bidirectional trajectory of TrkB-GFP in the dendrite is marked by gray arrowheads. (B) Histogram of TrkB-GrP trajectory velocities undergoing processive movement based on the analysis or 14 trajectories from 11 axons collected from 2 independent cultures. (C) Schem of surface-labeling setup used in experiments shown in (D) and (E). Neurons expressing SNAP-TrkB were labelec
with surface-SNAP dye to follow the localization and transport of TrkB from the plasma membrane. (D) BDNF induces the internalization and accumulation of cell-surface TrkB in intracellular compartments. Time-lapse images of surface-SNAP-TrkB-labeled cells treated with bath application of control media or media supplemented with BDNF. Images were taken at $\mathrm{t}=0,30$, and
axon and dendrites. Neurons were pulsed labeled with surface-SNAP then treated with BDNF or control media, then imaged at a time window of 45-90 min later. Neurofascin is imaged and presented as in (A). White arrowheads mark retrograde transport of internalized TrkB. (F) Scheme of compartmental microfluidic chamber (MFC) setup used in experiments shown in $(\mathrm{G})$ and $(\mathrm{H})$ depicted under (1) and (I-K) under (2). Neurons were plated in
the proximal compartment, with their axon projecting into the distal compartment. Surface-SNAP labeling was carried specifically in either the proximal or the distal axon compartment to follow axon-to-somAP labeling was transport. Control or BDNF supplemented media was added in the distal compartment. (G) BDNF induces somatic accumulation of retrogradely transported TrkB. Axons of SNAP-TrkB-expressing neurons were surface labeled in the distal axon and treated with either control or BDNF supplemented media for $2 \mathrm{~h}$ in the distal axon then fixed. mCherry was used to label neuron morphology. Arrowheads mark SNAP-TrkB puncta appearing in the distal axon in response to BDNF. (H) Scatter plot of mean \pm SEM of density of SNAP-TrkB spots counted in somas with $\mathrm{axons}$ crossing into the distal compartment after surface-SNAP labeling in the distal compartment. * *Student's t-test, Kymographs of surface-labeled SNAP-TrkB mobility along proximal and distal axon segments. Neurons cultured in MFC and transfected with SNAP-TrkB were surface labeled in the proximal compartment, followed by treatmen with either control or BDNF media in the distal compartment for 2-4 h before live imaging. (J) Scatter plot of mean \pm SEM trajectory velocity measured for individual SNAP-TrkB trajectories. (K) Cumulative frequency plots of mean trajectory velocity. Data in (I-K) are based on a pool of 42, 32, 54, and 41 trajectories from 6, 7, 8, and 10 axon segm 5 .

An important difference is that in contrast to TrkB-GFP, the axonal transport of the internalized SNAP-TrkB is exclusively retrograde. BDNF addition enhanced SNAP-TrkB transport in the axon, doing so without altering its overall retrograde bias (Fig 1E; Video S2).

The selectivity of axonal transport direction prompted us to further investigate the re-distribution of internalized TrkB receptors between the axonal and somatodendritic compartments of the neuron. To do this we utilized microfluidic compartmental chambers (MFCs) to specifically label and manipulate the distal axon and soma compartment. Dissociated hippocampal neurons were plated in the proximal compartment, and due to the length of the microchannels, only axons were able to traverse the distance and reach the distal compartment. We then used the MFC cultures to carry two separate experiment to follow either distal axon-to-soma or soma-to-distal axon re-distribution of internalized TrkB (Fig 1F, assay 1 and assay 2, respectively). As axonal stimulation with neurotrophin is known to activate both axon-to-soma and soma-to-axon transport of Trk receptors (Ascaño et al., 2009; Yamashita et al., 2017), we sought to examine how TrkB re-distribution is affected by application of BDNF specifically to the distal axon. To allow sufficient time for internalization and retrograde transport of axonal TrkB, BDNF incubation in the dista compartment was carried for $2 \mathrm{~h}$ immediately after surface-SNAP labeling in the distal axon. In BDNF treatment condition, SNAP-TrkB in the distal axon formed concentrated puncta along the axon and accumulated in the soma $(\mathrm{Fig} 1 \mathrm{G}, \mathrm{H})$, while in the control condition SNAP-TrkB remained diffused along the axon and did not accumulate in the soma. Thus, we confirm that in hippocampal neurons axonal TrkB undergoes internalization in dista axons, followed by retrograde transport and accumulation in the soma in response to ligand stimulation, in accordance with the retrograde signaling endosome model.

To follow anterograde re-distribution of TrkB into the axon, we carried surface labeling of SNAP-TrkB in the proximal compartment. BDNF treatment or control media was then promptly added to the distal compartment and the neurons were imaged in a time window of 3-4 h post-labeling. To follow SNAP-TrkB dynamics in the distal axon we imaged a segment of the axon that resided either at the distal compartment or in the microchanne within $100 \mu \mathrm{m}$ from its distal opening. For the proximal axon we selected segments that were both at a distance of $100 \mu \mathrm{m}$ from the soma and within the proximal compartment (hence these axonal segments were directly exposed to surface-SNAP labeling). We observed that proximally labeled TrkB receptors are rarely seen in the distal compartment, while in the proxima compartment more puncta can be observed (Fig 11 ). Proximally labeled TrkB puncta their mobility in both their mobility in both the distal and profile of proximally labeled TrkB is mainly retrograde in both proximal and distal segments in either control and in 3-4 h BDNF treatment in the distal axon compartment (Fig 1K) 

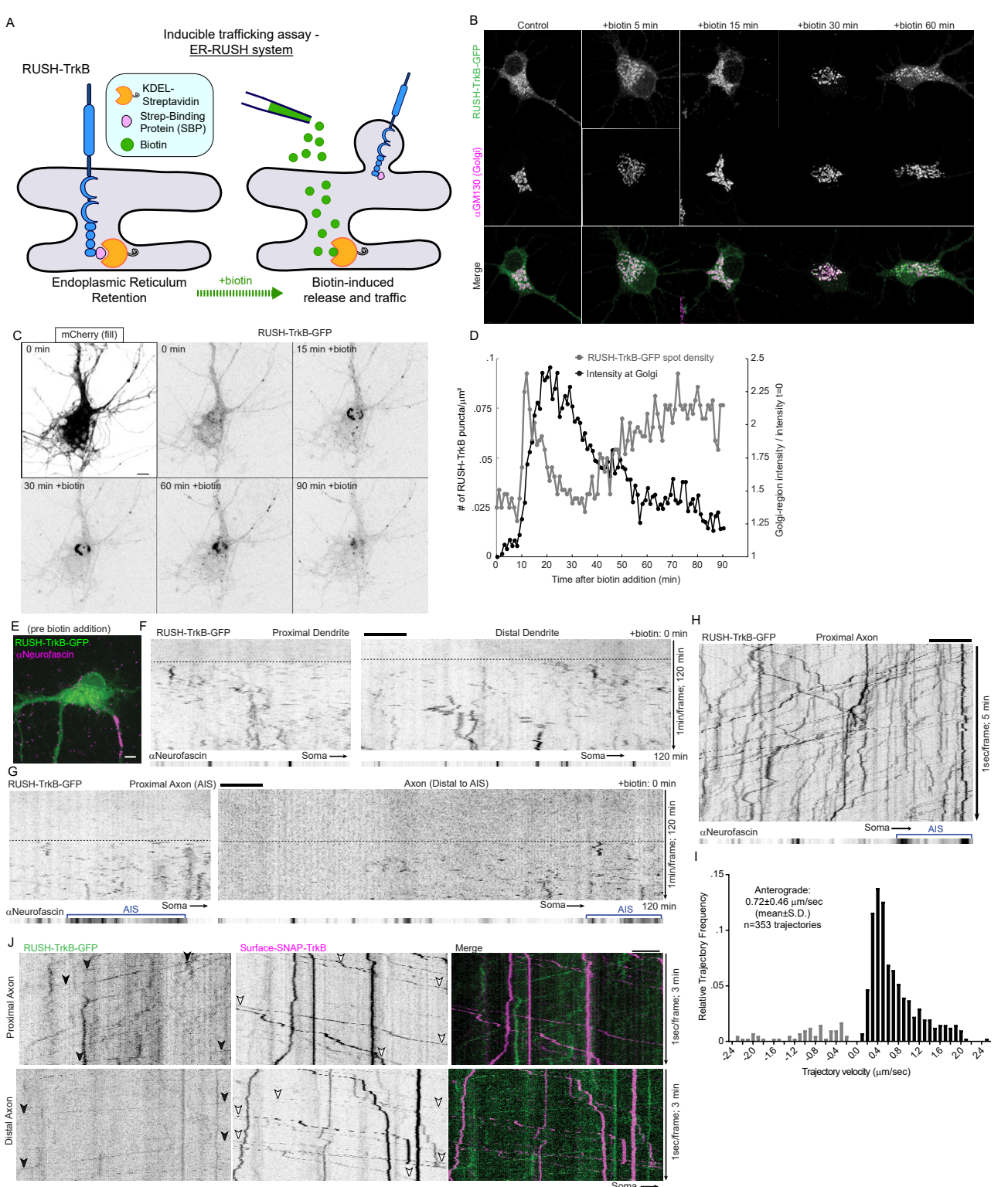

Figure 2. Anterograde transport of TrkB into the axon from the secretory pathway
(A) Scheme of the RUSH-based assay used in the experiments shown in this figure. Neurons were transfected
with RUSH-TrkB construct encoding a bi-cistronic mRNA for KDEL-streptavidin and an SBP-TrkB with tag. Temporal control of tagged TrkB-SBP trafficking from the ER is enabled by the addition of biotin which dislodges TrkB-SBP from KDEL-streptavidin, resulting in the release of TrkB for further trafficking. (B) Images of fixed neurons expressing RUSH-TrkB-GFP and immunostained with GM130 antibody after biotin addition. (C) Live imaging series of neurons co-expressing RUSH-TrkB-GFP and cytosolic mCherry following biotin addition. (D) Mean RUSH-TrkB-GFP signal in the peri-nuclear Golgi area (as ratio signal Soma of neuron expressing RUSH-TrkB and stained for neurofascin (AIS) imaged prior to biotin addition $(\mathbf{F}, \mathbf{G})$ Kymographs of RUSH-TrkB in segments along the axon (F). (G) Kymographs of RUSH-TrkB along a dendritic process. Kymographs in $(\mathrm{F})$ and $(\mathrm{G})$ were based on a single neuron imaged for $2 \mathrm{~h}$ after addition of biotin
at $1 \mathrm{~min} /$ frame. Dashed horizontal bars highlight the onset time in which discrete RUSH-TrkB aggregates appear.
(H) Kymograph of RUSH-TrkB-GFP transport in the proximal axon after biotin-induced release costained with neurofascin. Individual axon segments were imaged at a time window of 45-90 min after biotin for $5 \mathrm{~min}$ at s/frame. (I) Histogram of individual RUSH-TrkB-GFP trajectories velocities in the proximal axon. Data are of
353 trajectories from 20 individual axons from 3 independent cultures. (J) Kymograph of surface-labeled SNAPTrkB and RUSH-TrkB-GFP motilities along the same axon segments. Neurons co-expressing SNAP-TrkB and RUSH-TrkB-GFP were labeled with surface-SNAP then stimulated with BDNF to promote TrkB internalization and transport. Biotin was then added, and neurons were imaged for 3 min between 45-90 min later. Black and white arrowheads mark trajectories of RUSH-TrkB-GFP and SNAP-TrkB, respectively. Scale bars: $5 \mu \mathrm{m}$.

When BDNF treatment was carried for 1-2 $\mathrm{h}$, few processive anterograde transport events of proximally labeled SNAP-TrkB were observed (Fig S1D), possibly reflecting an axon-to-

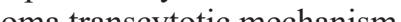
soma trest TrkB internalization, the endosomal TrkB is primarily sorted for retrograde transport in the TrkB internalization, the endosomal TrkB is primarily so

\section{Direct axonal trafficking of TrkB from the secretory pathway}

The predominance of retrograde transport of TrkB endosomes in the axon suggests that active delivery of receptors to the distal axon is via a different membrane trafficking pathway. We therefore turned our focus to the secretory pathway as a likely source for the anterogradely transported TrkB cargos. To specifically probe the trafficking and transport of TrkB originating at its site of biosynthesis, i.e., the ER, we used the inducibly released retention system (RUSH) (Boncompain et al., 2012). Briefly, we incorporated GFP- or SNAP-tagged TrkB into the KDEL-streptavidin-IRES-streptavidin-binding protein (SBP) vector. These constructs encode ER-sequestered KDEL-streptavidin and SBP conjugated TrkB tagged with GFP or SNAP (henceforth termed RUSH-TrkB-GFP and RUSH-SNAP-TrkB). Neurons were maintained with neutravidin to sequester biotin and prevent premature release of RUSHTrkB from the ER, which is controllably induced by the addition of excess biotin (Fig 2A). Incubation with neutravidin for $48 \mathrm{~h}$ and treatment with biotin in naive neurons did not induce detectable changes in ER and Golgi morphologies suggesting their secretory system is intact (Fig S1I). Indeed, addition of biotin to media of RUSH-TrkB-expressing neurons induced a shift of the receptor into the peri-nuclear region and its concentration with the cisGolgi protein GM130 in the soma within 15-30 min after treatment, followed by appearance of smaller TrkB carriers dispersed from the Golgi after 60 min (Fig 2B). Live imaging (a) colgi carriers, fllowed by concentration of RUSH-TrkB-GFP in a peri-nuclear region, normally within 10-30 min, then a dispersal from the Golgi concomitant with formation of post-Golgi carriers occurring within 30-45 min (Fig 2C,D; Video S3). RUSH-TrkB successfully traffics and fuses with the plasma membrane, as we observe an increase in the cell-surface signal of RUSH-TrkB-SNAP after treatment with biotin for $2.5 \mathrm{~h}$ (Fig S1E,F).

To test the functionality of RUSH-TrkB, we transfected human embryonic kidney (HEK) cells that lack endogenous TrkB with RUSH-TrkB-GFP and stimulated them with BDNF to follow downstream extracellular signal-regulated kinases 1\&2 (ERK1/2) signaling. Expression of RUSH-TrkB-GFP induced a BDNF independent ERK $1 / 2$ phosphorylation that is possibly due to ligand-independent kinase activity by overexpressed TrkB, while treatment with BDNF for 30 min activated a significant increase in ERK1/2 signaling in RUSH-TrkBGFP cells pre-treated with biotin for $2 \mathrm{~h}(\mathrm{Fig} \mathrm{S1G}, \mathrm{H})$. Thus, release of ER sequestration by biotin-induced proper RUSH-TrkB trafficking to the plasma membrane with intact BDNF receptor function.

Notably, RUSH-TrkB dynamics differed in axons versus dendrites. Both proximal and distal dendritic segments contained retained RUSH-TrkB before biotin addition and responded with fast re-distribution into discreet spots 20 min after biotin addition (Fig 2E,F, onset time is highlighted by dashed bars). Comparatively, axonal signal is minimal before biotin addition, and the appearance of distinct punctate signal in the proximal and distal axon occurs typically after 45-60 min (Fig $2 \mathrm{G}$, dashed bars). 

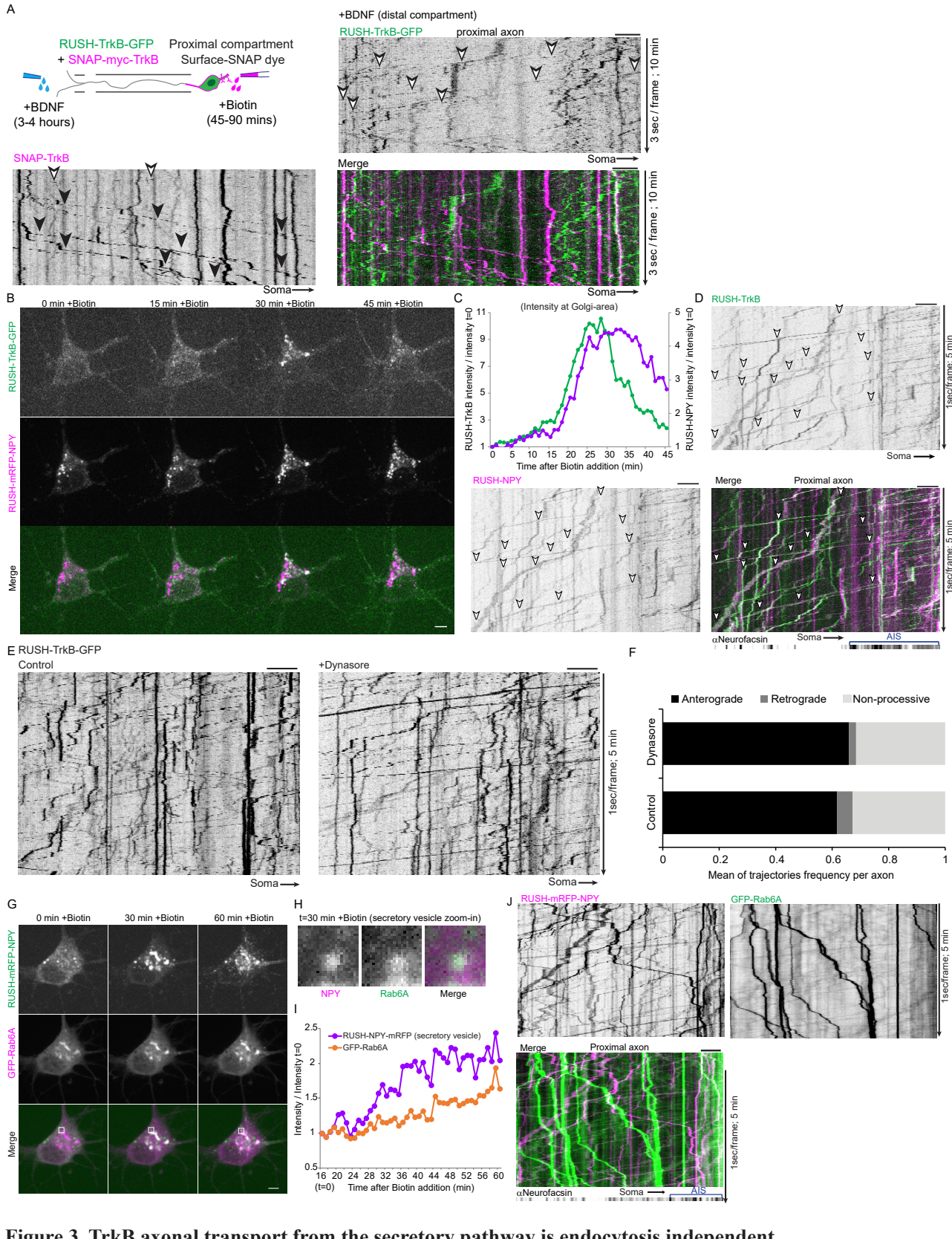

Figure 3. TrkB axonal transport from the secretory pathway is endocytosis independent

(A) RUSH-TrkB and SNAP-TrkB transport dynamics in proximal to distal transport of internalized SNAP-TrkB using microfluidic devices. Neurons were transfected with SNAP-TrkB+RUSH-TrkB-GFP as in Fig 2J, and surfaceSNAP labeling was applied to the proximal compartment, followed by BDNF addition (distal compartment) and then biotin (proximal compartment). Live imaging of axons in the proximal compartment was carried 3-4 $\mathrm{h}$ after BDNF addition at $3 \mathrm{~s} /$ frame for 10 min. Kymographs are representative of 2 independent experiments.
White and black arrowheads mark anterograde and retrograde transport of SNAP-TrkB or RUSH-TrkB-GFP (B,C) Neurons co-expressing RUSH-TrkB-GFP and RUSH-mRFP-NPY were treated with biotin and imaged for 45 min at 1 frame/min. (C) Representative plot of RUSH-NPY and RUSH-TrkB intensities in the peri-nuclear Golo region (normalized to $\mathrm{t}=0$ ) following biotin addition. (D) Secreted TrkB and NPY co-transport along the axon.
Neurons co-transfected as in $(\mathrm{B}, \mathrm{C})$ and were immunostained for neurofascin to label the AIS. RUSH-TrkBGFP and RUSH-mRFP-NPY were live imaged during a time window of 45-90 min after addition of biotin for $5 \mathrm{~min}$ at 1 frame/s. Kymographs of the proximal part of the axon show the co-transport of secreted NPY and or dynasore for 15 min before biotin was added to induce post-ER trafficking. (E) Proximal axons were imaged at a time window of $45-90 \mathrm{~min}$ after biotin for $5 \mathrm{~min}$ at $1 \mathrm{~s} /$ frame. (F) Kymograph trajectories were categorized and counted to measure transport dynamics in individual axons. Data are mean ratios of anterograde, retrograde, and non-processive trajectories per axon and are based on 11 (control) and 12 (dynasore) individual axons from 2 independent experiments. (G) Secreted NPY is trafficked and transported in Rab6 carriers. Neurons were co(H) Zoom-in of a plot of NPY and Rab6A intensities in the puncta in (I) during the course of biotin treatment Scale bars: 5 mime These results suggest that the trafficking of TrkB from the ER is restricted to the somatodendritic compartment and that TrkB is transported from the soma in post-Golgi carriers into the axon. Released RUSH-TrkB is transported almost exclusively in the anterograde direction toward the distalaxonatameanvelocity of $0.72 \mu \mathrm{m} / \mathrm{s}$, fitting withregulatedmotor-basedtransportmechanism (Fig 2H,I; Video S3). We further tested whether endocytosed TrkB can be co-transported with secretory TrkB in the same neuron by co-transfecting SNAP-TrkB and RUSH-TrkBGFP. After Surface-SNAP labeling, BDNF was added for 30 min to induce TrkB endocytosis, followed by biotin addition. Similar to results shown in Figure 1, the processive mobility of SNAP-TrkB was exclusively retrograde, while the RUSH-TrkB was transported separately in the anterograde direction both at the proximal and distal axon (Fig 2J; Video S4). Separate transport was also seen using the soma-to-axon transcytosis experimental paradigm (Fig $3 \mathrm{~A}$ ), further indicating that TrkB endosomal and secretory trafficking are diametrically regulated. Further probing of the TrkB sorting was carried by co-expressing ER-retained TrkB and neuropeptide Y (NPY) as a soluble cargo marker, which is also delivered into the axon. RUSH-TrkB and RUSH-NPY concomitantly accumulate in the Golgiupon trafficking induction and are then transported together toward the distal axon in the same carriers (Fig 3B-D). This affirms that the RUSH-TrkB carriers are sorted directly into the axon without an inter axs of the soluble NPY signal. Indeed, pre-incubation with dynasore, an inhibitor of dynamin-dependent endocytosis, signal. Indeed, pre-incubation with dynasore, an inhibitor of dynamin-dependent endocytosis,
did not affect RUSH-TrkB secretory trafficking and transport in the axon (Fig 3E,F). We therefore conclude that TrkB undergoes direct transport from the secretory system to the axon.

\section{TrkB trafficking to the axon is regulated by the Rab6 GTPase}

Direct trafficking of TrkB from the secretory pathway to the axon requires sorting of the receptor into anterograde-bound membrane compartment. Rab GTPase are master regulators of membranal compartment sorting and trafficking in the endosomal and secretory system (Pfeffer, 2017; Stenmark, 2009). We decided to test the association of secretory TrkB carrier with Rab27B, Rab3C, Rab8A, Rab6A, and Rab11A as markers for membranal compartments that were associated with anterograde axonal transport or with plasma membrane delivery of secretory pathway carriers (Arimura et al., 2009; Ascaño et al., 2009; Koseki et al., 2017; Wakana et al. 2012). For this, we co-transfected RUSH-TrkB-GFP with labeled Rab proteins. While neither Rab27B, Rab3C, Rab8A nor Rab11A co-localized with RUSH-TrkB (Fig 4), we found that RUSH-TrkB and Rab6A co-localized to a high extent in the perinuclear concomitant with RUSH-TrkB accumulation in the Golgi area after biotin addition (Fig 5A; Video S7). Similar co-localization of RUSH-TrkB was observed with endogenous Rab6 (Fig S2A). The spatiotemporal accumulation of RUSH-TrkB in the Golgi region is correlated with the recruitment of wild-type Rab6A, but not with GDP-locked Rab6AT27N mutant (Fig 5B). We found that Rab6A and RUSH-TrkB are accumulating together in discrete puncta also outside the Golgi area (Fig 5C,D, highlighted by yellow box). Accordingly, we found that a major fraction of anterograde RUSH-TrkB carriers were coCA the a the distaxon (Fig 5E, depicted by arrowheads; Video S5), while co-transport of RUSH-TrkB and Rab6A is dependent on the GTP-binding state of Rab6A, 

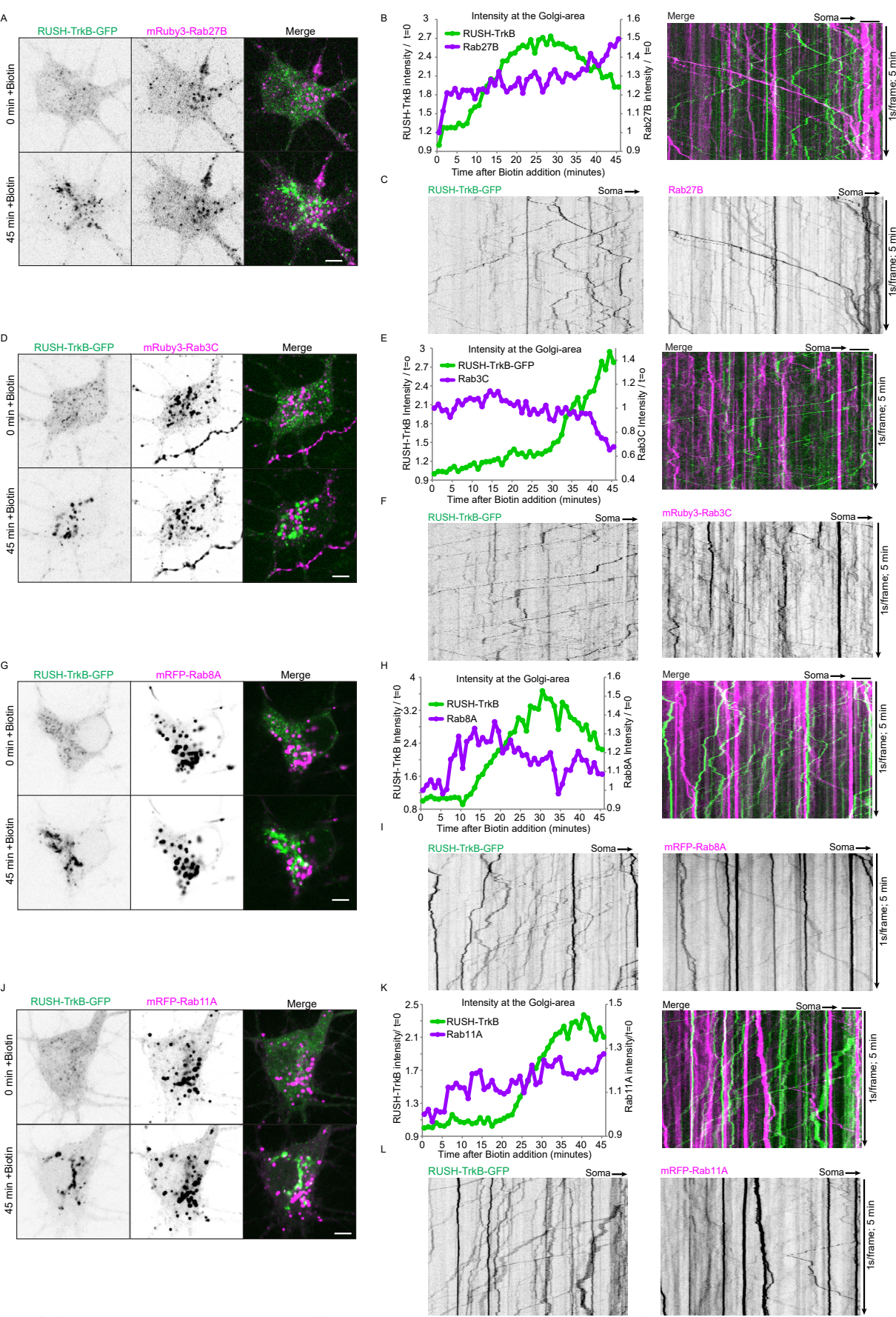

Figure 4.Anterogradely transported RUSH-TrkB carriersare negative for Rab27B, Rab3C,Rab8A, and Rab11A (A-L) Neurons co-expressing RUSH-TrkB-GFP and labeled Rab proteins. (A-C) mRuby3-Rab27B, (D-F)
mRuby3-Rab3C, (G-I) mRFP-Rab8A, and (J-L) mRFP-Rab11A. Neurons were live imaged in the soma are immediately after addition of biotin for $45 \mathrm{~min}$ at 1 frame/min $(\mathrm{A}, \mathrm{D}, \mathrm{G}, \mathrm{J})$, and then we imaged for $5 \mathrm{~min}$ at 1 s/frame at the proximal axon during a time window of 45-90 min after addition of biotin (C,F,I,L). Plots show RUSH-TrkB and Rab proteins intensities in the peri-nuclear Golgi region over time in representative neurons
(B,E,H,K). Kymographs of RUSH-TrkB-GFP and respective Rab proteins motility in individual axons (C,F,I,L). as both wild-type and GTP-locked Rab6A-Q72L mutant co-localized with RUSH-TrkB, while the GDP-locked Rab6A-T27N mutant did not (Fig 5E,F). Rab6A was also recruited to and transported with RUSH-NPY carriers in the proximal axon (Fig 5G-5I), suggesting that Rab6A regulates post-Golgi trafficking and axonal sorting in neurons. Importantly, we observe a distinct co-transport of wt-Rab6 A with anterogradely transported TrkB-GFP, but not with retrograde TrkB-GFP(Fig S2C,D). Thus, Rab6 likely has a specific role in regulating anterograde axonal trafficking of TrkB, as well as confirming that the mechanisms of TrkB axonal trafficking are not altered by the RUSH system.

To effectively disrupt Rab6 functions we depleted both Rab6A and Rab6B proteins in neurons using shRNA (Fig S2A). Depletion of Rab6 disrupted RUSH-TrkB trafficking, causing either a block in the Golgi following the early phase of ER-to-Golgi re-distribution (termed "blocked") or a retrograde flow from condensates at the Golgi back to diffuse ER signal (termed "frustrated") (Fig 5G,H; Video S6). The ratio of neurons displaying successful, frustrated, or blocked secretion process after 45 min of biotin treatment shows that Rab6 (As a previous study suggested the role of Rab27B in anterograde axon transport of TrkB (Arimura et al 2009), we sought to re-examine whether it plays a regulatory role in TrkB trafficking and transport using shRNA against Rab27A and Rab27B. In agreement with our observation that secretory pathway derived TrkB is not associated with Rab27B and in contrast with Rab6 depletion, we found no effect of Rab27 knockdown on secretory trafficking and axon transport of released RUSH-TrkB (Fig S2E,G).

KIF5C and KIF1A regulate formation and axonal transport of TrkB carriers Kinesin-1 and kinesin-3 families are the major drivers of anterograde axonal transport of various cargos (Maday et al., 2014). For a non-biased identification of specific kinesins that interact with TrkB, we applied an imaging-based split-kinesin assay previously described (Jenkins et al 2012). We co-transfected hippocampal neurons with FRB-conjugated kinesin cargo-binding tail domain tagged with Myc as bait (KIF-td-FRB), TrkB-GFP as the probed cargo and an cargo and this assay, assocition between kinsin tall domain and TrkB-GFP results in accumulation of the receptor in axon tips following rapalog-induced FKBP-FRB coupling (Fig 6A,B) We initially tested TrkB-GFP distribution in neurons expressing KIFIA-td-FRB and KIF5C, observing TrkB-GFP enrichment in the axon tips following rapalog addition (Fig 6C). We therefore systematically tested kinesin tail domains interaction with TrkB by quantifying the ratio between mean TrkB-GFP signal at the axon tip and its respective soma for different kinesin tail domains and thus identified significant TrkB interaction for the KIF5C kinesin-1 motor (Fig 6D) and for the kinesin-3 motors: KIF1A, KIF1B $\beta$, and KIF16B (Fig 6E) We also tested several kinesin- 2 and kinesin- 4 family members, observing a positive interaction TrkB-GFP accumulation, we confirmed that upon addition of rapalog all the KIF tail domains examined were successfully recruited to the axon tips (Fig S3B). Notably, while some KIF-td constructs were expressed at lower amounts relative to TrkB-GFP in comparison with others Fig S3C), these differences could not explain positive or negative interaction results with TrkB-GFP-e.g., KIF5A-td and KIF5B-td both were expressed efficiently but did interact with TrkB, while KIF13B-td and KIF1A-td were similarly expressed, however, only KIF1A-td showed clear interaction with TrkB (Fig 6D,E).

To validate the identified TrkB-kinesin interactions we carried a streptavidin pull-down in HEK cells by co-expressing Bio-tagged kinesin tail domain (Bio-Kiftd), BirA biotin ligase, and TrkB-GFP. In accordance with the split-kinesin-positive interaction, Bio-KIF5C-td and Bio-KIF1A-td efficiently interacted with TrkB-GFP (Fig S4A). KIF1B 3 and KIF16B showed weak and barely detectable TrkB binding, respectively, while KIF5A, KIF5B, and KIF21B co-precipitated with TrkB but did not associate with the receptor in the neuronal split-kinesin assay (Fig 6D,E; Fig S4A). 

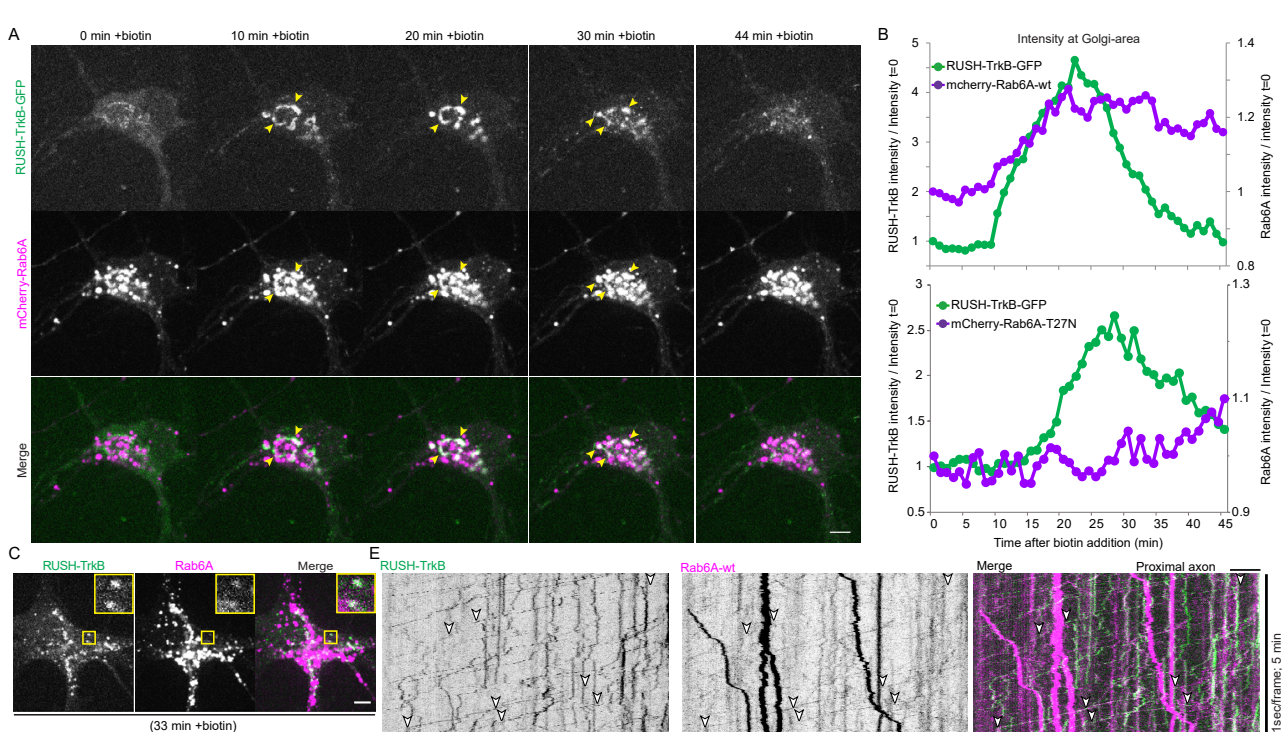

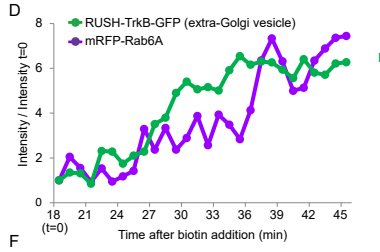
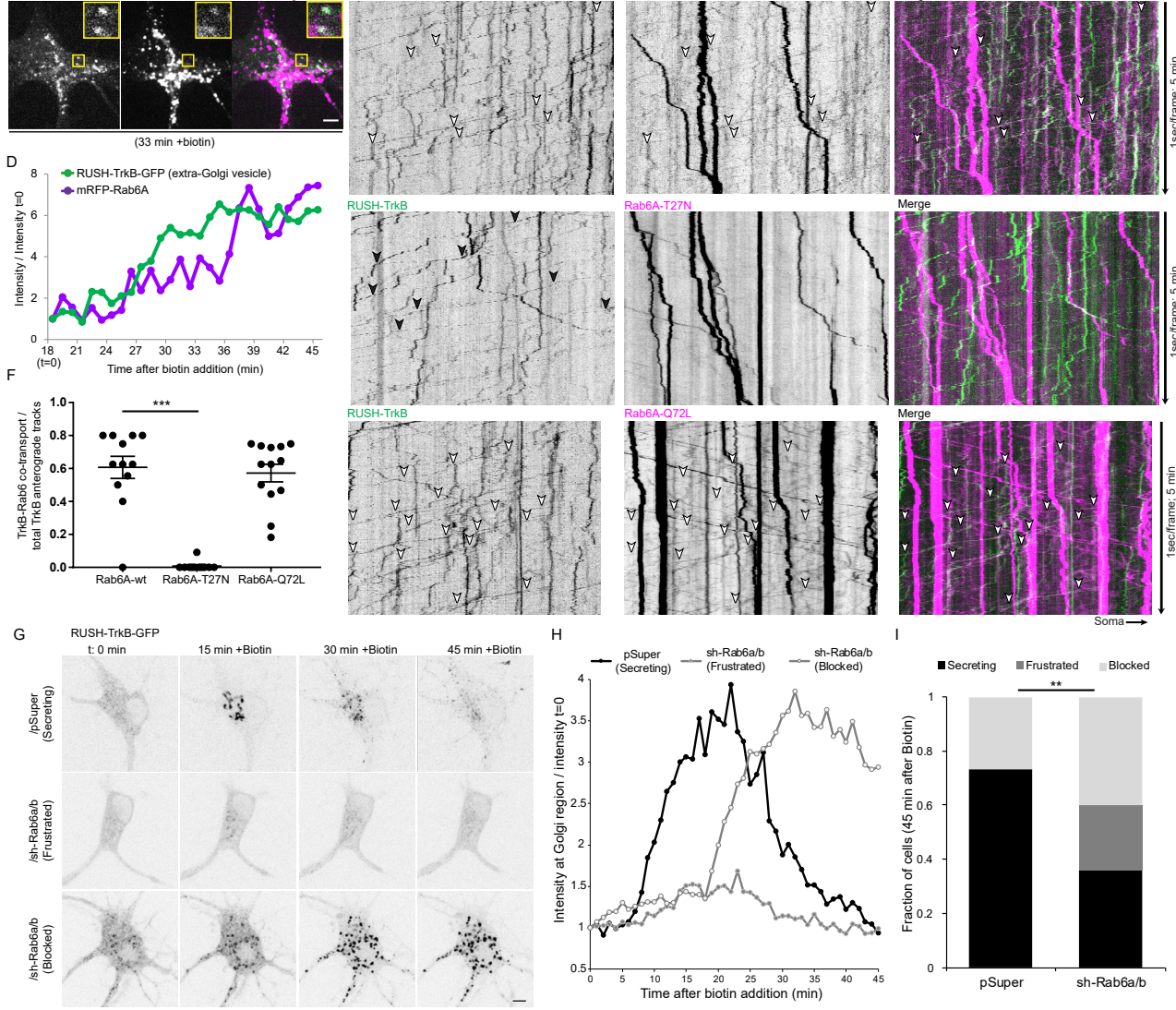

igure 5. Trafficking and anterograde transport of secreted TrkB in Rab6-positive compartments (A-D) Neurons co-expressing RUSH-TrkB-GFP and mCherry-Rab6A-WT or Rab6A-T27N were imaged in the biotin. Arrowheads mark RUSH-TrkB and Rab6 A co-localization in the Golgi region. (B) Comparative intensity plof of RUSH-TrkB and Rab6A-WT or Rab6A-T27N in the peri-nuclear Golgi region (normalized to $t=0$ ) in two separate neurons. (C) A zoom-in of RUSH-TrkB and Rab6A in discrete tubular (upper images) and punctate (lower images) compartments, taken from the time series shown in (A). (D) Time plot of the intensity of RUSH-TrkB and Rab6A at a somatic punctum shown in (C) following its formation and up until $45 \mathrm{~min}$ after addition of biotin. (E,F) Neurons co-
expressing RUSH-TrkB and indicated Rab6A constructs were immunostained against neurofascin to label the AIS and time series were captured at a time window of $45-90$ after addition of biotin, for 5 min each at 1 frame/s. (E) Kymographs of RUSH-TrkB and Rab6A motility in proximal axons. White and black arrowheads mark Rab6A-positive (WT and Q72L) and -negative (T27N) RUSH-TrkB carriers. (F) Ratios of anterograde TrkB traces $\mathrm{p}<0.001, \mathrm{n}=12,11$, and 13 axons of Rab6A-WT, Rab6A-T27N and Rab6A-Q72L conditions, respectively, from 3 independent cultures each. (G-I) Neurons were transfected with RUSH-TrkB-GFP and either vector (pSuper) or shRNA constructs targeting Rab6A and Rab6B. (G) Live image series of RUSH-TrkB-GFP in the soma following biotin addition. Neurons were manually categorized as secreting, frustrated, or blocked based on the profile of TrkB secretion in response to biotin in the course of 45 min. (H) Time plot of RUSH-TrkB intensity in the Golgi region (normalized to $t=0$ ) of neurons expressing either pSuper or sh Rab6 from different categories in (G). (I) Frequencies pSuper and shRab6 conditions, respectively, from 4 independent cultures each. Scale bars: $5 \mu \mathrm{m}(\mathrm{A}, \mathrm{G}), 2 \mu \mathrm{m}$ (C) To validate and further examine the interactions between KIF5C, KIF1A, and TrkB we carried a mass-spectrometry-based proteomic analysis using Bio-Kif-td as bait. We probed the interactome of Bio-KIF5C-td, Bio-KIF1A-td in HEK cells either expressing TrkB-GFP or GFP alone and used Bio-KIF17-td in TrkB-GFP expressing cells as a negative control, as KIF17-td did not interact with TrkB according to both split-kinesin and pull-down assays. The full list of detected proteins in the different pull-down conditions and their peptide spectrum matches (PSM) value is presented in Table S1. In accordance with the split-kinesin and western blot assays, we find higher spectral counts and fold-enrichment of TrkB peptides (PSM) in KIF1A and KIF5C compared with KIF17 and normalized to GFP-bait control (Fig S4B,C). Expected interactors of Bio-KIF5C-td and Bio-KIF1A-td, respectively, e.g., MAP7family proteins and calmodulin were unaffected by TrkB co-expression. An additiona proteomic analysis of KIF5C, KIF1A, and KIF16B tail domain baits confirmed that KIF5Cand KIF1A-specific interactors were not altered by TrkB co-expression (Fig S4D; Table S1)

To further test the function of KIF5C and KIF1A in the anterograde axonal trafficking of TrkB, we used shRNA to deplete either motor in neurons co-transfected with ER-to-Golgi trafficking of released RUSH-TrkB, which accumulated in the peri-nuclea egion similar to the control. Nevertheless, KIF5C depletion blocked the dispersal of RUSH TrkB carriers from the Golgi area into discrete carriers, while depletion of KIF1A did no affect ER-to-Golgi and post-Golgi trafficking, which was similar to control (Fig 6G,H; Video S7). While vesicular carriers of RUSH-TrkB formed normally in KIF1A-depleted neurons, their anterograde transport in the proximal axon was inefficient, evident by a reduction in the ratio of processive anterograde-bound vesicles, and more pausing and bidirectiona trajectories (Fig 6I,J; Video S8). Altogether these data show that the kinesin-1 motor KIF5C and kinesin-3 motor KIFIA co-operate in the formation and transport of TrkB carriers from the secretory compartment into and along the axon.

\section{DISCUSSION}

In this study, we visualized TrkB receptor trafficking and transport in hippocampal neurons and describe the molecular mechanisms that control its delivery in the axon. We showed that endocytosed TrkB undergoes retrograde transport in the axon, in accordance with the established model of the neurotrophin signaling endosome (Cosker and Segal, 2014). We also found that anterogradely transported TrkB is not derived from endosomal compartments. kinesin-1 KIF5C and kinesin-3 KIF1A motor proteins.

\section{Direct versus indirect trafficking of Trk receptors into the axon}

TrkA receptors were previously shown to take an indirect, transcytotic route in SCG neurons from somatodendritic membrane to the distal axon via recycling endosomes (Ascaño et al., 2009; Yamashita et al., 2017). In comparison, we found that the transport of endocytic TrkB in the axons of hippocampal neurons is almost entirely retrograde and that BDNF stimulation in the axon enhances the overall transport flux without affecting this retrograde bias. 

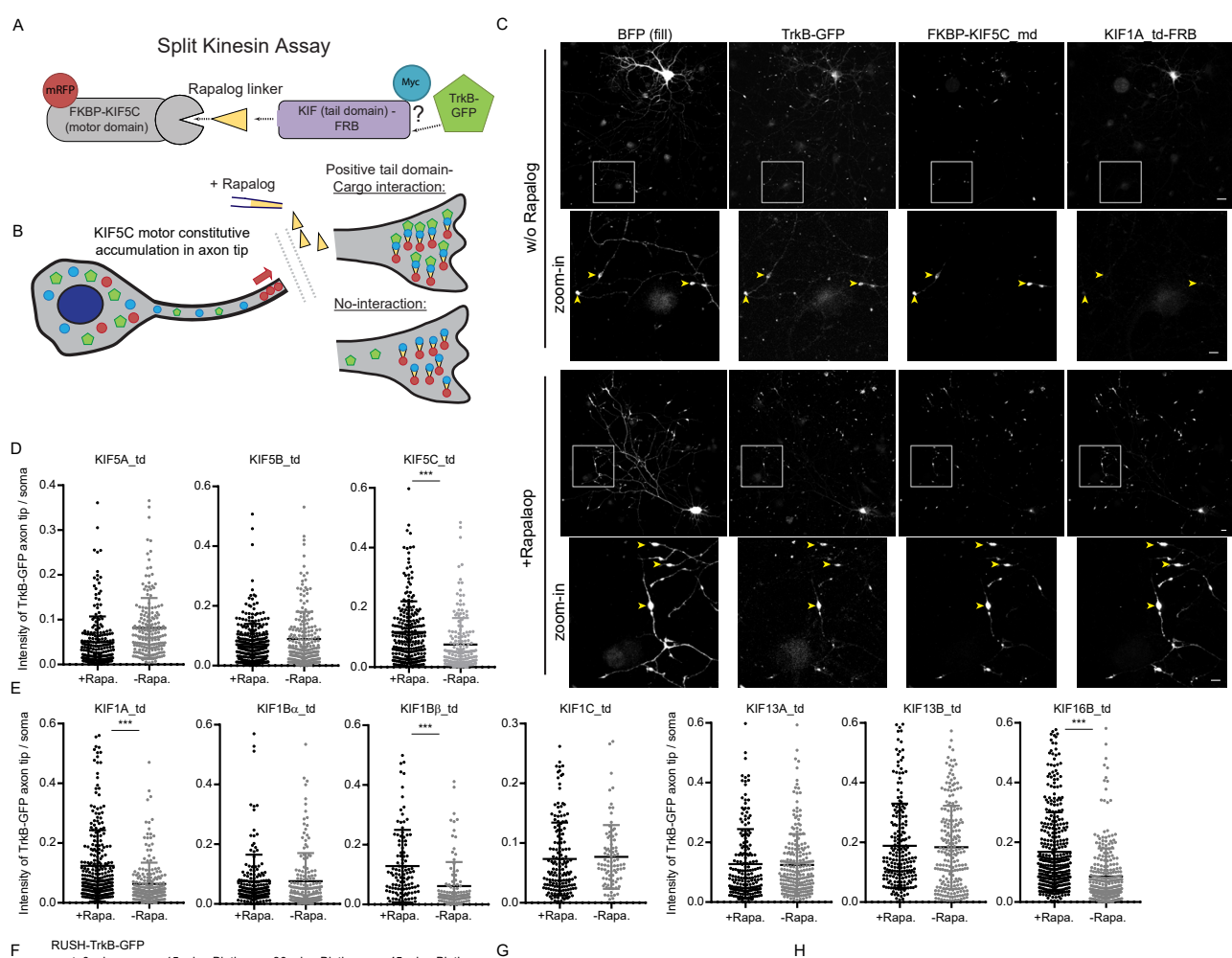

KFI3A_L
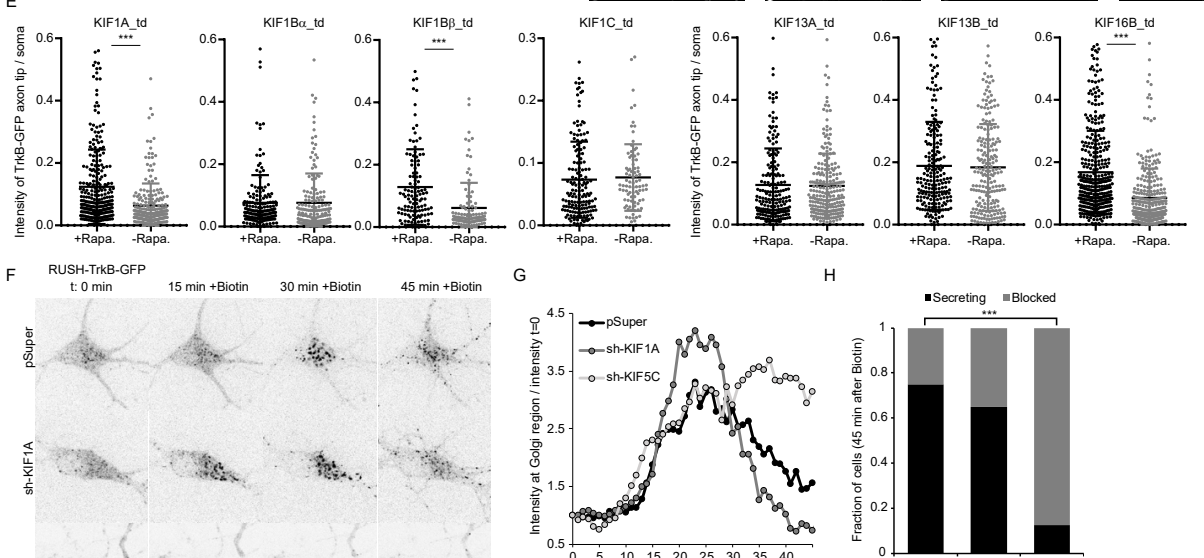

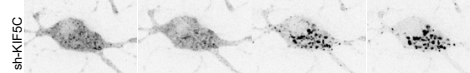
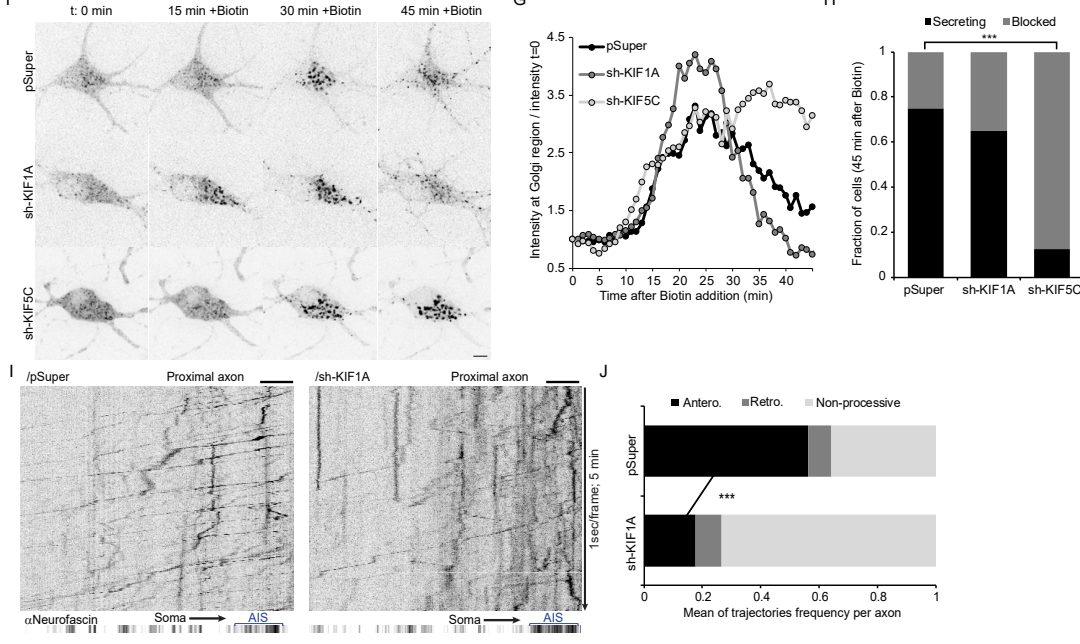

Figure 6. The kinesins KIF5C and KIF1A regulate the axonal trafficking of TrkB

(A,B) Schemes of the split-kinesin assay (SKA) used in the experiments presented in (C-E). (A) The components of the SKA with TrkB-GFP as cargo reporter. (B) Rapalog addition induces the mobilization of the kinesin tail domain and accumulation at the axon tips due to tight interaction with the constitutively motile motor KIF5C. Interaction between the kinesin tail domain and the TrkB cargo will result in accumulation of TrkB in the axon tips together with the KIF-td and KIF5C-md where rapalog is added. (C-E) Neurons were transfected with SKA components described immunostaining for myc-tagged KIF-td. (C) Images of the soma and axon tips (highlighted in zoom-in insets) of neurons assayed for TrkB-KIF1A interaction with and without rapalog addition. Scale bars, 10 and 5 mm (insets). (D) SKAresults for TrkB-GFP and Kinesin-1 family. (E) SKA results for TrkB-GFP and Kinesin-3 family. Data in (D-E) are TrkB-GFPsignal in individual axon tips, normalized to the signal in the soma of each axon tip. Lines depict mean and SD ***Two-sided Student's $t$-test, $p<0.001$. Data points were collected from the following number of axon tip:
KIF5A: 221 and 181; KIF5B: 303 and 214; KIF5C: 239 and 176; KIF1A: 270 and 219; KIF1Ba: 167 and 162 ;
KIF1BB: 118 and 110; KIF1C: 158 and 86; KIF13A: 187 and 230; KIF13B: 204 and 208: and KIF16B: 357 and 288 for +Rapa and -Rape KInditions, respectively. (F-H) Neurons co-transfected with RUSH-TrkB-GFP and shRNA Constructs a $45 \mathrm{~min}$ at 1 frame/min. (F) Time series images of representative neurons expressing RUSH-TrkB-GFP with specified shRNA constructs. (G) Time plots of RUSH-TrkB intensity at the Golgi (normalized to $t=0$ ) of neurons shown in (F). (H) Frequency of neuron categorized as secreting or blocked in response to $45 \mathrm{~min}$ of biotin. $* * *$ Chi-square test $\mathrm{p}<0.001, \mathrm{n}=28,20$, and 16 neurons from 5,3 , and 3 experiments for pSuper, sh-KIF1A, and sh-KIF5C conditions, respectively. (I,J) Neurons expressing RUSH-TrkB-GFP and pSuper or ShKIF1A were stained for neurofascin (AlS). Time-lapse imaging was carried in a time window of $45-90 \mathrm{~min}$ after addition of biotin. Images series were Frequency of trajectory directionality in the proximal segment of the axon. Trajectories with net displacement of less than $15 \mu \mathrm{m}$ in the course of $5 \mathrm{~min}$ were counted as non-processive. The processive traces were classified based on their directionality as either retrograde or anterograde. Bars depict mean ratio of trajectories per each time series (axon). trajectories of pSuper and sh-KIF1A conditions, respectively, from 3 independent experiments. Scale bars: $5 \mu \mathrm{m}$.

Interestingly, soma-to-axon transport of internalized TrkB was more apparent after short BDNF stimulation of the distal axon, suggesting that long-distance signaling induces TrkB transcytosis, at least to some extent. Polarized sorting of endosomal cargos in the axon may differ between types of neurons and during their maturation, which may explain the sparsity of anterograde transcyctotic transport of TrkB in hippocampal axons compared with that of TrkA in SCG neurons. In DRG and SCG neurons Rab11-positive recycling endosomes are transported into the axon and regulate insertion of membrane proteins in the growth cone (Ascaño et al., 2009; Eva et al., 2010). In contrast, CNS neurons display a shift in Rab11 trafficking dynamics during neuronal development, from localization throughout the neuron

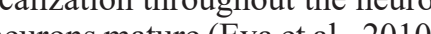
These differences in endosomal trafficking mechanisms merit further investigation, as they may underlie differences in axon growth and regeneration capacity in the PNS versus CNS in developing and mature neurons. (Fawcett, 2020).

\section{Axonal trafficking of proteins in the secretory pathway}

TrkB is a glycoprotein membrane receptor and as such undergoes N-linked glycosylation in the ER and subsequent maturation in the Golgi before it is transported to the plasma membrane (Haniu et al., 1995; Rajagopal et al., 2004; Watson et al., 1999). Accordingly, here, we show that TrkB's axonal trafficking follows a canonical course within the secretory pathway. The receptor leaves the ER and re-distributes in discrete compartments in dendrites and soma, which is followed by its transition into the somatic Golgi. The immediate redistribution into discrete puncta in dendrites prior to as well as concomitant with accumulation in the Golgi suggests that post-ER trafficking of TrkB takes place in neurites-however, we did not assess whether these events represented Golgi-independent trafficking to the plasma membrane proteins, TrkB was found to also be inserted into the plasma membrane without undergoing Golgi-dependent trimming of its glycan chains, suggesting that it can take a Golgi-independent route to the plasma membrane via ER-to-plasma membrane carriers such as Rab11 (Bowen et al., 2017; Hanus et al., 2016). While this trafficking route is mostly linked to local synthesis and trafficking in dendrites, it was also speculated that a similar Golgi-bypass route could be utilized for local biosynthesis of certain membrane proteins in axons (González et al., 2018). Nevertheless, considering that in CNS neurons the secretory organelles (i.e. ribosomal ER, ERGIC, and Golgi) are confined to the somatodendritic domain and (i.e sparse evidence for local C. vast majority of the menbrane protein cargos designated to the distal axon originate from the somatodendritic-localized secretory compartments and are transported there by microtubulebased motors, as our data show in the case of TrkB (Farías et al., 2015; González et al., 2018 Krijnse-Locker et al., 1995; Maday et al., 2014) 


\section{Motor-cargo interactions for anterograde axonal transport of TrkB}

Here, we found that the Rab6 small GTPase regulates the formation of post-Golgi TrkB carriers and that its GTP-binding state controls their formation and subsequent transport into the distal axon. While early studies ascribed Rab6 to regulate retrograde Golgi-to-ER trafficking (Martinez et al., 1994; White et al., 1999), more recent studies established its role in the exocytic pathway (Fourriere et al., 2019; Grigoriev et al., 2007, Grigoriev et al., 2011; Schlager et al., 2010). Our data suggest that Rab6 is a master regulator of trafficking in the secretory pathway, particularly for post-Golgi axon-bound cargos. Rab6 has been shown to interact with both anterograde and retrograde motor proteins (Lee et al., 2015; Matanis et al., 2002). In HeLa cells, KIF5B kinesin-1 motor was described to drive Rab6 vesicle transport from the Golgi to the plasma membrane (Grigoriev et al., 2007). Here, we found that the formation post-Golgi Rab6-positive vesicles containing TrkB is dependent on kinesin-1 motor KIF5C. Kinesin-1 was previously shown to be critical for TrkB anterograde transport in hippocampal neurons via a Rab27B-dependent mechanism (Arimura et al., 2009). However, we did not find Rab27B to associate nor to regulate TrkB direct transport of TrkB into the axon. This discrepancy could result from the use of more mature DIV7-11 neurons in our experiments, compared with DIV3 neurons, whose Ftial segment (AIS) is still maturing and therefore could differ in their axonal sorting mechanisms in which Rab27B participates. Also, we used inducible release RUSH system to focus on TrkB transport directly from the secretory pathway, which we identified as the prominent mechanism for axonal delivery of TrkB, while they had imaged the total TrkB signal, which may represent different endomembranal compartments whose anterograde axon transport is regulated by Rab27B. Here, we show that KIF5C is required for the formation of discrete secretory TrkB compartments but is yet not sufficient to drive TrkB transport beyond the initial part of the proximal axon. Our data indicate that TrkB trafficking to the distal axon also depends on KIF1A motor activity. We suggest that distinct kinesin family members differentially regulate axonal TrkB trafficking. Interestingly, a recent study dissected the collaborative roles of the kinesin-1 and kinesin-3 pair KIF5B and KIF13B in peripheral transport of secretory Rab6 carrier in HeLa cells (Serra-Marques et al., 2020). A previous work from our lab described mechanism for selective cargo axon entry in DRG neurons that is based on the coordinated action of the coordinated action of kinesin- 1 and kinesin- 3 motors at the pre-axonal filtering zone (Gumy et al, 2017). That study showed that the microtubule-associated protein MAP2, which localizes at the pre-axonal zone, inhibits microtubule binding of kinesin-1 but not of KIF1 motors, hence, requiring association of KIF1 motors on the cargo for its proper transport into the axon. While the structures of the pre-axonal zone and the initial segment of DRG and hippocampal neurons are altogether different, our two studies suggest that the cooperative action of kinesin- 1 and kinesin- 3 motors is a general mechanism for axona delivery. KIF1A was recently shown to have weaker binding to GTP-tubulin that is needed for KIF1A synaptic vesicle precursor cargo release at pre-synaptic sites, which are rich in GTP-tubulin, dynamic microtubules (Guedes-Dias et al., 2019). It is intriguing to speculate KIF1A binding and processive transport.

In summary, our study proposes a model for the anterograde transport of TrkB by direct trafficking from the biosynthetic system to the axon in Rab6 carriers that is driven by the mutual activity of two kinesin motors: kinesin-1 KIF5C, which is required for the trafficking and transport of secretory carriers, and kinesin-3 KIF1A that facilitates passage through the AIS and processive transport along the axon.

\section{AUTHOR CONTRIBUTIONS}

E.E.Z. initiated the study, designed and performed experiments, analyzed and formatted data for presentation in the manuscript, and wrote the manuscript. J.J.A.H. established experimental procedure, performed experiments, and guided experimental work.
Y.H. and C.B. performed experimental work. R.S. performed the mass spectrometry experiment and analysis. M.A. gave valuable advice on mass spectrometry; C.C.H. designed the overall experimental plan, supervised the research, and wrote the manuscript. All authors contributed to the formatting and editing of the manuscript.

\section{MATERIALS AND METHODS}

Animal handling

Pregnant Wistar Rat dames at day 18 of pregnancy were supplied from Janvier Labs (France) at the same day the were used for preparing neuronal cultures. Handling and euthanization of the animals were performed in compliance and were approved by the Animal Ethical Review Committee (DEC) of Utrecht University. The hippocampa cultures where prepared from groups of 7-14 embryos of undetermined sex in each independent experiment.

Cell line and primary hippocampal neurons cultures
HEK293T (HEK) cells were maintained in DMEM media supplemented with FBS $10 \%$ and Penicillin-Streptomycin $1 \%$. Cultures were kept in humidified incubator at $37^{\circ} \mathrm{C}$ and $\mathrm{CO} 5 \%$. Cells were passaed by vicorous pipetting and re-plated at 1:10 density every 3-4 days and kept at sub-confluent density.

Primary hippocampal neuron culture was prepared from E18 rat brains using a modified protocol based on the procedure of Kaech \& Banker (Kaech and Banker, 2006). Dissociated hippocampal neurons were plated on $18 \mathrm{~mm}$ coverslips coated with poly-L-Lysine $(37.5 \mathrm{\mu g} / \mathrm{ml}$ ) and (laminin $5 \mu \mathrm{g} / \mathrm{ml})$ without a glial feeder layer. For normal cultures on cover slips, 100,000 viable cells were seeded on $18 \mathrm{~mm}$ coverslip placed in 12 -well multiwe plate, resulting in a cell density of $28,000 / \mathrm{cm}^{2}$. Neurons were maintained in Neurobasal media supplemented with B27. $2 \%$, Glutamine $0.5 \mathrm{mM}$, Glutamate $15.6 \mu \mathrm{M}$ and Penill maintained in humidified incubator at $37^{\circ} \mathrm{C}$ and $\mathrm{CO}_{2} 5 \%$

Antibodies, probes and reagents

The following antibodies and probes were used in Immunofluorescence (IF) and Western-Blot (WB) assays at the specified dilutions: Neurofascin, IF: 1:250; TRIM46, IF:1:500; GM130, IF: 1:500; RTN4a (NOGO), IF: 1:500; WB: 1:100 used at 1.250-1:1000 dilution. For WB, secondary antibodies conjugated with IRdye680LT- or IRdye800CW were used at 1:10000 dilution, for detection of biotinylted proteins, Streptavidin-IR680LT (Li-Cor) was used at 1:10000 dilution. Surface SNAP-dyes (New England Biolabs) were dissolved at $2 \mathrm{mM}$ concentration in DMSO and was freshly diluted to $1 \mu \mathrm{M}$ for labeling. BDNF was reconstituted at $10 \mu \mathrm{g} / \mathrm{ml}$ and was applied to cells at a $50 \mathrm{ng} / \mathrm{ml}$ concentration. Dynasore was dissolved in DMSO at stock concentration of $60 \mathrm{mM}$ and applied at a concentration of $60 \mathrm{uM}$. Compartmental neuronal culture in Microfluidic Chamber (MFC)

Polydimethylsiloxane (PDMS) microfluidic devices were produced in-house using a mold designed by the Saudou lab for in-vitro cortico-striatal connectivity studies (Virlogeux et al., 2018). Briefly, the MFC was composed of 3 compartments: proximal, intermediate and distal compartments (respectively corresponding to cortical, synaptic and striatal in the original paper). Two arrays of parallel microchannels separated the compartments, a 500um long

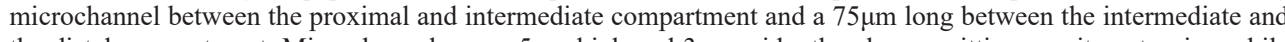
the distal compartment. Microchannels were $5 \mu \mathrm{m}$ high and $3 \mu \mathrm{m}$ wide, thereby permitting neurite extension while blocking the passage of cell somas. MFC devices were attached to $25 \mathrm{~mm}$ glass coverslips and then coated with Polythe proximal cominin as specified above. Following hippocampal neuron preparation, 50,000 cells were seeded in axons could cross it and reach the intermediate and distal compartments. As we used the device to compartmentalize the distal axon from the somatodendritic domains, we refer here to both the intermediate and distal compartments as the distal compartment, and refer to the section of the axon $250 \mu \mathrm{m}$ and further from the proximal opening of the $500 \mu \mathrm{m}$-long microchannel as the distal axon.

DNA constructs for protein and shRNA expression PGW-CM was used as an expression backbone vector unless otherwise specified. ShRNA constructs were cloned in TrkB CDS (NTRK2) into pEGFP-N1 backbone and gifted by R. Segal (Harvard University). SNAP-myc-TrkB was cloned using Gibson assembly by fusing into pGW1 the following fragments: TrkB signal-peptide, myc-SNAP-tag fusion from myc-SNAP-mGluR2 (gifted by Harold McGillavry, Utrecht University) and the rest of TrkB CDS from TrkB-GFP. RUSH-TrkB-GFP, RUSH-SNAP-TrkB and RUSH-mRFP-NPY were cloned by inserting PCR amplified TrkB-EGFP CDS (from TrkB-GFP), SNAP-TrkB CDS (from SNAP-myc-TrkB) and mRFP-NPY CDS (from previously in-house constructed N1-mRFP-NPY plasmid) into Sbfl/Xbal restricted IRES3-STR-KDEL-SBPEGFP-Ecadherin (Boncompain et al., 2012

GFP-Rab6A and mCherry-Rab6A were previously cloned in-house by inserting human Rab6A CDS into 
Q72L-Fw: ACAGCAGGTCTAGAGCGGTTC, Q72L-Rev: GTCCCATAATTGCAATCGTAC, T27N-Fw: GTTGGAAAGAACTCTTTGATCACCAGATTCATGTATGACAG andT27N-Rev: GCTTTGCTCCCCCAGGAA mRuby3-Rab3C and mRuby3-Rab27B were constructed by cloning Rab3C CDS (from in-house cloned GW1-GFPRab3C) and Rab27B CDS (from GFP-Rab27B, Addgene \# 89447) into BglII/BamHI restricted mRuby3-Tubuli (Addgene \# 74256); mRFP-Rab11A, mRFP-Rab8A was previously constructed in-house by inserting respective
human Rab CDS into pGW1-mRFP backbone.

Constructs for Split-Kinesin Assay were prepared as follows: mRFP-KIF5Cmd-FKBP was cloned by
fusing mRFP CDS, human KIF5C (aa 1-559) CDS and FKBP sequences in pGW1 backbone. Kinesin-tail domin constructs were prepared by fusing into pGW1 a FRB-3xMyc sequence with respective human kinesin CDS fragments: KIF5A (aa 375-1032), KIF5B (aa 374-963), KIF5C (aa 376-955), KIF17 (aa 376-1028), KIF3A (a 386-702), KIF1A (aa 395-1698), KIF1B $\alpha$ (aa 390-1153), KIF1Bß (aa 390-1770), KIF1C (aa 395-1103), KIF13A (a 397-1770), KIF13B (aa 443-1826), KIF16B (aa 395-1266), KIF4A (aa 374-1129), KIF4B (aa 374-1234), KIF21 A (aa 377-1661) and KIF21B (aa 410-1624). Biotin-tagged kinesin-tail domains were prepared by inserting the sequences, without the FRB-3xMyc sequence into a modified pBio-GFP backbone (previously made in house by addition of $\mathrm{E}$. Coll BitA bioth-ligase recoge lion seque into pEOF CI (Schatz, 1993)) resulting in Bio-KIFconstructs without GFP, FRB and Myc tags. Plasmid for BirA expression was previously formed by subcloning Bir from the original pEV-P

shRNA constructs against rat Rab27A and Rab27B were based on published siRNA sequences (Arimura et al., 2009): CCAGTGTACTGTACCAGTA (Rab27A) and CAACATTTCTTACCGATA (Rab27B), which were inserted into pSuper vector. The
shRab6B (Schlager et al., 2010); shKIF5C (Pan et al., 2019) and shKIF1A (Kevenaar et al., 2016).

\section{Transfection of protein expression and shRNA constructs}

Hippocampal neurons cultured on coverslips and in MFC were transfected on days in-vitro (DIV) 7-10 post culturing using Lipofectamine. A total of $1.8 \mu \mathrm{g}$ DNA was used for the transfection of each sample (coverslip/MFC). The ratios of the different constructs in the mix were varied depending on the specific assays, which are further detailed in their

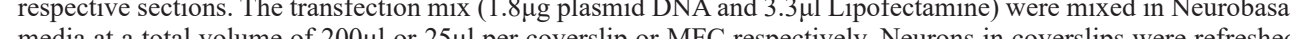
media at a total volume of $200 \mu \mathrm{l}$ or $25 \mu \mathrm{l}$ per coverslip or MFC respectively. Neurons in coverslips were refreshed with Neurobasal media supplemented only with Glutamine. The mix was incubated for $30 \mathrm{~min}$ then applied on mix of $1: 1(\mathrm{v} / \mathrm{v})$ of fresh Complete NBM and conditioned media (removed from the samples before transfection). For neurons transfected with RUSH constructs, the post-transfection incubation media was supplemented with Neutravidin. Neurons were then incubated for $1-3$ days before being assayed, depending on specific experiments Polyethylenimine (PEI) at a ratio of 3:1 ( $\mathrm{\mu l} / \mathrm{\mu g}$ ) PEI/DNA. HEK cells were replaced with DMEM only media before transfection, and the transfection mix was incubated in DMEM for 15 min before applying on cells. For assaying ERK1/2 phosphorylation, HEK cells in 12-well plates transfected with 1 1 g DNA of either pCDNA3-GFP, TrkBGFP and RUSH-TrkB-GFP. For kinesin tail-domain pulldown cells were transfected with a mix of BirA $(4 \mu \mathrm{g})$

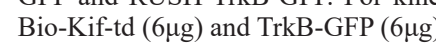

\section{Retention Using Selective Hooks (RUSH) assay}

In order to deplete extracellular biotin before experimental induction of ER-release, complete Neurobasal media 作 induce trafficking from the $\mathrm{ER}$, media was replaced with normal growth media supplemented with biotin at $40 \mu \mathrm{M}$. Immunofluorescent labeling in fixed cells

Neurons were fixed by incubating in Paraformaldehyde 4\% + Sucrose 4\% in PBS for 25 min at room-temperature and then washed $3 x$ in PBS. Samples were placed in humidified dark chamber. Cells were permeabilized in Trito $X-1000.2 \%$ in Blocking solution (BSA $1 \%$ in PBS) for 5 min in room temperature, then washed out and incubated with Blocking solution for $15 \mathrm{~min}$ at $4^{\circ} \mathrm{C}$. Primary antibody was added and incubated for $60 \mathrm{~min}$ at $37^{\circ} \mathrm{C}$ for sanples on covers ips or overnight at $4^{\circ} \mathrm{C}$. and replaced with secondary antibody which was incubated for $60 \mathrm{~min}$ at $37^{\circ} \mathrm{C}$ (for coverslips) or $120 \mathrm{~min}$ at roomFluoro Surfering

Cell surface expression of SNAP-TrkB was probed of SNAP-TrkB in room-temperature, followed by 2 washouts in NBM. Cells were fixed and secondary mouse IgG, 1:200) was applied for 30 min followed by 3 washouts with PBS. Labeling of intracellular SNAPTrkB was done by permeabilizing for $5 \mathrm{~min}$, blocking for $15 \mathrm{~min}$ at $37^{\circ} \mathrm{C}$, and incubating with myc antibody (1:200) for $30 \mathrm{~min}$ followed by 3 washouts and labeling with Alexa-488-anti mouse IgG. For internalization assay, a pulse labeling with myc antibody $(1: 200)$ was carried for $15 \mathrm{~min}$ at $37^{\circ} \mathrm{C}$. Neurons were then treated with control or BDNF supplemented media for 30 min followed by fixation. Alexa-647-anti mouse IgG was applied for 30 min prior to permeabilization to label SNAP-TrkB that remained at the cell surface. Samples were then Confocal imaging of surface (Alexa-647) and internalized (Alexa-488) was carried along the volume of the soma.
To quantify the total signal, a Sum slices projection was carried and the mean 647 and 488 signals at the soma region per neuron were

Confocal imaging of fixed cells

Zeiss LSM700 microscopes were used to perform confocal imaging of fixed cells. Samples were imaged with $60 \mathrm{x}$ oil-immersion NA 1.4 objective equipped with automated stage. Gain and laser power was adjusted accordin to intensity to obtain non-saturated signals. Zoom level were adjusted to reach a resolution of 90-110 nm /pixe and $z$-stack images were taken at $0.5 \mu \mathrm{m}$ intervals. For imaging of neurons in compartmental SNAP-TrkB labeling experiments, only labelled neurons with axons crossing through the microchannels from proximal to dista neurons and their axons extensions from the proximal to the distal compartments and along the microchannels.

Live-cell confocal imaging

Live-cell confocal imaging
Live neurons were mounted on coverslip holders and placed under a Nikon Ti microscope inside a Tokai HIT on-stage Live neurons were mounted on coverslip holders and placed under a Nikon Ti microscope inside a Tokai HIT on-stage objective (NA 1.4) and Nikon Perfect Focus System were used in all experiments. Individual neurons were located using widefield fluorescence imaging via the eyepieces. Confocal imaging was obtained using Yokogawa CSU-XI unit and images were captured with either EMCCD or sCOMS cameras. Control of microscopy system, imagin protocol and image capture was done with Metamorph software. EMCCD gain was set to 950 in all channels. Laser was adjusted to obtain sufficient emission signal for tracking of individual vesicles in all imaging channels while minimizing phototoxicity. Imaging of neuronal transport was carried at 1 frame per second. Imaging of RUSH trafficking after biotin-induced release was cartied at 1 min per frame. Dual-color imaging was done by sequentia switching between 488 \& $561 \mathrm{~nm}$ laser excitation and fast GFP and mCherry filter. Multi-position imaging of RUSH trafficking and SNAP-TrkB internalization was controlled via automated stage.

\section{Imaging based Split-Kinesin Assay}

Neurons at DIV 7-10 were transfected with a combination of TrkB-GFP, mRFP-KIF5C-md-FKBP and variable tagged Kinesin tail domain (FRB-3xMyc-KIF-td). Neurons were incubated with either normal culture media as
control or media supplemented with rapalog $100 \mathrm{nM}$. Cells were fixed 16-24 $\mathrm{h}$ after transfection and processed for immunofluorescent labeling for myc-tag detection. Samples where imaged using Nikon Eclipse Ni80 upright fluorescent microscope equipped with CoolSNAP HQ2 CCD (Photometrics). Axon tips were imaged based on focal accumulation of KIF5C-md signal, and respective somas were imaged by tracing TrkB-GFP signal. Image analysis was carried using Fiji interest (ROH) wer manully marked on axon tips and corresponding somas. The average signal of KIF-td and TrkB-GFP signal was then measured and used to calculate the tip/soma enrichment ratio.

\section{Preparation of HEK cell lysates for Pull-down and Western Blot (WB)}

HEK cells were harvested 16-24 $\mathrm{h}$ after transfection by vigorous pipetting. Cell suspension was centrifuged at $700 \mathrm{~g}^{\circ}$ for $1 \mathrm{~min}$, then washed once with cold PBS and spun again. For direct WB analysis of cell lysate, pellet was lysed in RIPA buffer: $\mathrm{NaCl} 150 \mathrm{mM}$, TX-100 1\%, sodium deoxycholate (SDC) $0.5 \%$ and sodium dodecyl sulfate (SDS) $0.1 \%$, Tris $50 \mathrm{mM}$, pH 8.0 supplemented with protease and phosphatase Inhibitors. For pull-down experiments, a
milder detergent buffer was used instead: $\mathrm{NaCl} 150 \mathrm{mM}$, TX-100 $1 \%$ and Tris $25 \mathrm{mM} \mathrm{pH} 8.0$. Cells were mixed $10 \mathrm{x}$ by pipetting and incubated in the lysis buffer for $5 \mathrm{~min}$ on ice then centrifuged $1000 \mathrm{xg}$ ' for 5 ' at $4^{\circ} \mathrm{C}$. Cleared supernatant lysate was collected into a fresh tube and used for pull-down or direct analysis. For SDS-PAGE, samples were mixed 1:3 with Sample Buffer x4 (Tris 500mM pH 6.8, SDS 8\%, glycerol 2.5\%, DTT 10mM and bromophenol Blue), then heated to $95^{\circ} \mathrm{C}$ for $5^{\prime}$.

\section{Pull-down assay for Kinesin-tail domain and TrkB interactio}

Wif-td was incubated with Dynabeads Streptavidin blocking buffer (Tris $20 \mathrm{mM} \mathrm{pH} 8 . \mathrm{KCl} 150 \mathrm{mM}$, BSA $10 \mathrm{~cm}$ plate of lysed HEK cells) were pre-incubated with beads for $1 \mathrm{~h}$ at $4^{\circ} \mathrm{C}$ and the beads were washed 3 times with wash buffer (Tris $25 \mathrm{mM} \mathrm{PH} 8.0, \mathrm{NaCl} 150 \mathrm{mM}$, TXheated to $95^{\circ} \mathrm{C}$ for $5 \mathrm{~min}$

SDS-PAGE and Western Blot assay apparatus (Bio-Rad). Membranes were blocked in BSA $1 \%$ and incubated with primary antibodies for $2 \mathrm{~h}$ in roomtemperature or overnight at $4^{\circ} \mathrm{C}$. Secondary antibodies conjugated with IRdyes (Li-Cor) were incubated for $2 \mathrm{~h}$ in room temperature. Blots were scanned with Odyssey and analyzed with Fiji Gel Analyzer application.

Proteomic analysis of Kinesin-tail domain interactome

Beads were re-suspended in $20 \mu \mathrm{L}$ of Laemmli Sample buffer (Biorad) and supernatants were loaded on a 4-12\% gradient Criterion XT Bis-Tris precast gel (Biorad). The gel was fixed with 40\% methanol/10\% acetic acid and the stained for $1 \mathrm{~h}$ using colloidal coomassie dye G-250 (Gel Code Blue Stain Reagent, Thermo Scientific). After in-ge digestion (Stucchi et al. 2018), all samples were analyzed on a Orbitrap Q-Exactive Plus mass spectrometer (Thermo Fisher Scientific, Brenon, Germany) coupled to an Agilent 1290 Infinity LC (Agilent Technologies). Peptides were 
at a maximum pressure of 800 bar and chromatographically separated over the analytical column (Zorbax SB-C18, $1.8 \mu \mathrm{m}, 40 \mathrm{~cm} \times 50 \mu \mathrm{m}$; Agilent) using $90 \mathrm{~min}$ linear gradient from $7-30 \%$ solvent B $(0.1 \%$ formic acid in acetonitrile $)$ at a flow rate of $150 \mathrm{~nL} / \mathrm{min}$. The mass spectrometer was used in a data-dependent mode, which automatically switched between MS and MS/MS. After a survey scan from 350-1500 m/z the 10 most abundant peptides wer subjected to HCD fragmentation. For data analysis, raw files were processed using Proteome Discoverer 1.4 (versio (version 2.5.1. Matrix Science, UK) on the Human Uniprot database. Carbamidomethylation of cysteines was set as a fixed modification and oxidation of methionine was set as a variable modification. Trypsin was set as cleavage specificity, allowing a maximum of 2 missed cleavages. Data filtering was performed using percolator resulting in $1 \%$ false discovery rate (FDR). Additional filters were search engine rank 1 and mascot ion score $>20$. To infer protein abundancy of each individual protein co-purified with the bait protein, we relied on total numbers of Peptide Spectrum Matches (PSM). Crapome (Mellacheruvu et al., 2013) was used to analyze interacting binding proteins. The Fold Change calculation (FC-B, Crapome; by averaging the spectral counts compared to GFP-bait control) an PSM values were used for data visualization. Bubble plot of PSM and fold-enrichment presentation was created for selected proteins using custom made R script.

\section{Statistical analysis and data visualization}

All statistical details including the definitions of $n$, numbers of $n$ and statistical tests performed can be found in each figure and figure legends. No statistical methods were used to pre-determine sample sizes. Data distribution was assumed to be normal but this was not formally tested. Statistical testing was perfromed using unpaired Student analyzed and visulized analyzed and visualized using Excel (Microsoft) and Prism (Graphpad) software, except for where otherwise noted
Kymograph preparation and analysis of transport dynamics

Live imaging time-lapse series of transport dynamics were processed using Fiji. Image series were backgroun segmented line ROI tool and Kymographs were created using KymoResliceWide plugin (https://github con ekatrukha/KymoResliceWide). To measure velocity of traces, straight line ROIs were manually traced along linea segments of individual trajectories. The angle of the lines was used to calculate the average velocity of the mobility segments. To analyze the processivity of secreted RUSH-TrkB cargos along the axon, segmented line ROIs were traced. Trajectories that could be traced for 30 seconds or longer, or that crossed the entire distance of the traced axon were selected for further analysis. Trajectories covering more than $15 \mu \mathrm{m}$ in the duration of imaging were counted as either retrograde or anterograde, depending on the vector of movement, while trajectories that were immobile or
erratic were counted as non-processive.

\section{REFERENCES}

Arimura N., Kimura T., Nakamuta S., Taya S., Funahashi Y., Hattori A., Shimada A., Ménager C., Kawabata S.,
Fujii K. (2009). Anterograde transport of TrkB in axons is mediated by direct interaction with Slp1 and Rab27. Dev.

Ascaño M., Richmond A., Borden P., Kuruvilla R. (2009). Axonal targeting of Trk receptors via transcytosis Bentley M.,BankerG.(2016). The cellularmechanisms thatmaintainneuronal polarity. Nat. Rev. Neurosci. 17,611-622. Boncompain G., Divoux S., Gareil N., De Forges H., Lescure A., Latreche L., Mercanti V., Jollivet F., Raposo G., Bothwell M. (2016). Recent advances in understanding neurotrophin signaling. F1000Res. 5, 1885 .

Bowen A.B., Bourke A.M., Hiester B.G., Hanus C., Kennedy M.J. (2017) Golgi-Independent secretory trafficking through recycling endosomes in neuronal dendrites and spines. eLife 6, e27362.

Britt D.J., Farías G.G., Guardia C.M., Bonifacino J.S. (2016). Mechanisms of polarized organelle distribution in

Brummelkamp T.R., Bernards R., Agami R. (2002). A system for stable expression of short interfering RNAs in
mammalian cells. Science 296, 550-553.

Cosker K.E., Segal R.A.(2014). Neuronal signaling through endocytosis. Cold Spring Harb. Perspect. Biol. 6, a020669. De Boer E., Rodriguez P., Bonte E., Krijgsveld J., Katsantoni E., Heck A., Grosveld F., Strouboulis J. (2003). mice. PNAS 100, 7480-7485.

Eva R., Dassie E., Caswell P.T., Dick G., Ffrench-Constant C., Norman J.C., Fawcett J.W. (2010). Rab11 and its effector Rab coupling protein contribute to the trafficking of $\beta 1$ integrins during axon growth in adult dorsal roo ganglion neurons and PC12 cells. J. Neurosci. 30, 11654-11669.

Farías G.G., Guardia C.M., Britt D.J., Guo X., Bonifacino J.S. (2015). Sorting of dendritic and axonal vesicles at the pre-axonal exclusion zone. Cell Rep. 13, 1221-1232.

Fawcett J.W. (2020) The struggle to make CNS axons regenerate: why has it been so difficult? Neurochem. Res.

Fourriere L., Jimenez A.J., Perez F., Boncompain G. (2020). The role of microtubules in secretory protein transport.
Fourriere L., Kasri A., Gareil N., Bardin S., Bousquet H., Pereira D., Perez F., Goud B., Boncompain G., Miserey-
Lenkei S. (2019). RAB6 and microtubules restrict protein secretion to focal adhesions. J. Cell Biol. 218, 2215-2231. González C., Cornejo V.H., Couve A. (2018). Golgi bypass for local delivery of axonal proteins, fact or fiction? Curr.

Grigoriev I., Splinter D., KeijzerN., WulfP.S., Demmers J., Ohtsuka T., Modesti M., Maly IIV., Grosveld F., Hoogenraad
C.C., Akhmanova A. (2007). Rab6 regulates transport and targeting of exocytotic carriers. Dev. Cell 13 13,305-314

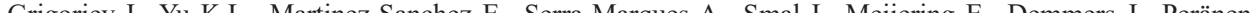
Pasterkamp R.J., van der Sluijs P. (2011). Rab6, Rab8, and MICAL3 cooperate in controlling docking and fusion of
Prigon exocytotic carriers. Curr. Biol. 21, 967-974.

Guedes-Dias P., Nirschl J.J., Abreu N., Tokito M.K., Janke C., Magiera M.M., Holzbaur E.L.F. (2019). Kinesin--3 responds to local microtubule dynamics to target synaptic cargo delivery to the presynapse. Curr. Biol. 29, 268-282. Gumy L.F., Katrukha E.A., Grigoriev I., Jaarsma D., Kapitein L.C., Akhmanova A., Hoogenraad C.C. (2017). MAP2 defines a pre-axonal

Haniu M., Talvenheimo J., Le J., Katta V., Welcher A., Rohde M.F. (1995). Extracellular domain of neurotrophin -glycosylation sites, and ligand binding. Arch. Biochem. Biophys. 322, 256-264. Hanus C., Geptin H., Tushev G., Garg S., Alvarez-Castelao B., Sambandan S., Kochen L., Hafner A.S., Langer J.D.,
Schuman E.M. (2016). Unconventional secretory processing diversifies neuronal ion channel properties. eLife 5, e20609.

Harrington A.W., Ginty D.D. (2013). Long-distance retrograde neurotrophic factor signalling in neurons. Nat. Rev.

Hoogenraad C.C., Milstein A.D., Ethell I.M. (2005). GRIP1 controls dendrite morphogenesis by regulating EphB

Huang S.H., Wang J., Sui W.H., Chen B., Zhang X.Y., Yan J., Geng Z., Chen Z.Y. (2013). BDNF-dependent recycling facilitates Tris

Jenkins B., Decker H., Bentley M., Luisi J., Banker G. (2012). A novel split kinesin assay identifies motor protein that interact with distinct vesicle populations. J. Cell Biol. 198, 749-761.

Kaech S., Banker G. (2006). Culturing hippocampal neurons. Nat. Protoc. 1, 2406-2415.

Kennedy M.J., Hanus C. (2019). Architecture and dynamics of the neuronal secretory network. Annu. Rev. Cell Dev.

Kevenaar J.T., Bianchi S., Van Spronsen M., Olieric N., Lipka J., Frias C.P., Mikhaylova M., Harterink M., Keijzer N., Wulf P.S. (2016). Kinesin-binding protein controls microtubule dynamics and cargo trafficking by regulating

Koseki H., Donegá M., Lam B.Y.H., Petrova V., van Erp S., Yeo G.S.H., Kwok J.C.F., Ffrench-Constant C., Eva R.,
Fawcett J.W. (2017). Selective rabl1 transport and the intrinsic regenerative ability of CNS axons. eLife 6, e26956. Krijnse-Locker J, Parton R.G., Fuller S.D , Griffths G, Dotti C.G. (1995). The organization of the endoplastic reticulum and the intermediate compartment in cultured rat hippocampal neurons. Mol. Biol. Cell 6, 1315-1332. Lasiecka Z.M., Winckler B. (2011). Mechanisms of polarized membrane trafficking in neurons - focusing in on endosomes. Mol. Cell. Neurosci. 48, $278-287$.

Lazo O.M., Gonzalez A., Ascaño M., Kuruvilla R., Couve A., Bronfman F.C. (2013). BDNF regulates Rab11mediated recycling endosome dynamics to induce dendritic branching. J. Neurosci. 33, 6112-6122.

Lee P.L., Ohlson M.B., Pfeffer S.R. (2015). The Rab6-regulated KIF1C kinesin motor domain contributes to Golg

Maday S., Twelvetrees A.E., Moughamian A.J., Holzbaur E.L.F. (2014). Axonal transport: cargo-specific
mechanisms of motility and regulation. Neuron 84, 292-309. Martinez O., Schmidt A., Salaméro J., Hoflack B., Roa M., Goud B. (1994). The small GTP-binding protein rab6 functions in intra-Golgi transport. J. Cell Biol. 127, 1575-1588.

Matanis T., Akhmanova A., Wulf P., Del Nery E., Weide T., Stepanova T., Galjart N., Grosveld F., Goud B., De Zeeuw C.I. (2002). Bicaudal-D regulates COPI-independent Golgi-ER transport by recruiting the dynein-dynacti

Mellacheruvu D., Wright Z., Couzens A.L., Lambert J.P., St-Denis N.A., Li T., Miteva Y.V., Hauri S., Sardiu M.E. for affinity purification-mass spectromethy data. Nat.

Pan X., Cao Y., Stucchi R., Hooikaas P.J., Portegies S., Will L., Martin M., Akhmanova A., Harterink M., Hoogenraad the. (2019). MAP7D2 localizes to
the aell Rep. 26, 1988-1999.

Park H., Poo M.M. (2013). Neurotrophin regulation of neural circuit development and function. Nat. Rev. Neurosci. $14,7-23$

Pfeffer S.R. (2017). Rab GTPases: master regulators that establish the secretory and endocytic pathways. Mol. Biol. Rajagopal R., Chen Z.Y., Lee F.S., Chao M.V. (2004). Transactivation of Trk neurotrophin receptors by G-protein-

Sampo B., Kaech S., Kunz S., Banker G. (2003). Two distinct mechanisms target membrane proteins to the axonal 
Schatz P.J. (1993). Use of peptide libraries to map the substrate specificitit of a peptide-modifying enzyme: a 13
residue consensus peptide specifies biotinylation in Escherichia coli. Biotechnology $111,1138-1143$.

Schlager M.A., Kapitein L.C., Grigoriev I., Burzynski G.M., Wulf P.S., Keijzer N., de Graaff E., Fukuda M. bicaudal-D-related protein 1 ( BICDR-1) regulates neuritogenesis. EMBO J. 29, 1637 -1651.

Scott-Solomon $E_{\text {.. }}$ Kuruvilla R. (2018). Mechanisms of neurotrophin trafficking via Trk receptors. Mol. Cell.
Neurosci 91 25.33 Serra-Marques A., Martin M., Katrukha E.A., Grigoriev I., Peeters C.A., Liu O., Hooikaas P.J., Yao Y., Solianova
V., Smal I. (2020). Concerted action of kinesin-1 KIF5B and kinesin-3 KIFI3B promotes efficient transport of exocytotic vesicles to microtubule plus ends. eLife 9 , e61302

Song M., Giza J., Proenca C.C., Jing D., Elliott M., Dincheva I., Shmelkov S.V., Kim J., Schreiner R., Huang S.-H Stenmark H. (2009). Rab GTPases as coordinators of vesicle traffici. Nat. Rev Mol. Cell Biol. 10, 513-52.

Stucchi R., Plucinska G., Hummel J.J.A., Zahavi E.E., Guerra San Juan I., Klykov O., Scheltema R.A., Altelaar DCV binding and liprin- $\alpha$ TANC2 recruits DCVs to postsynaptic sites. Cell Rep. 24, 685-700.

Tanaka Y, Niwa S Dong 4 Farkhondeh A. Wang $L$, Zhou R., Hirokawa N. (2016). The molecular motor KIF IA transports the TrkA neurotrophin receptor and is essential for sensory neuron survival and function. Neuron 90 $1215-1229$

Valenzuela J.I., Jaureguiberry-Bravo M., Salas D.A., Ramírez O.A., Cornejo V.H., Lu H.E., Blanpied T.A., Couve
A. (2014). Transsport along the dendritic endoplasmic reticulum mediates the trafficking of GABAB receptors. J. Sci. $127,3382-3395$.

Virlogeux A., Moutaux E., Christaller W., Genoux A., Bruyère J., Fino E., Charlot B., Cazorla M., Saudou F.

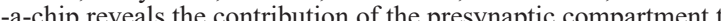
Huntington's disease. Cell Rep. 22, 110-122. Wakana Y., van Galen J., Meissner F., Scarpa M., Polishchuk R.S., Mann M., Malhotra V. (2012). A new class of
carriers that transport selective cargo from the trans Golgi network to the cell surface. EMBO J. 31, 3976-3990. Watson F.L., Porcionatto M.A., Bhattacharyya A., Stiles C.D., Segal R.A. (1999). TrkA glycosylation regulates receptor tocalizanis

White J., Johannes L., Mallard F., Girod A., Grill S., Reinsch S., Keller P., Tzschaschel B., Echard A., Goud B., Stelzer Wisco D., Anderson E.D., Chang M.C., Norden C., Boiko T. F Filsch H., Winckler B. (2003). Uncovering multiple
axonal targeting pathways

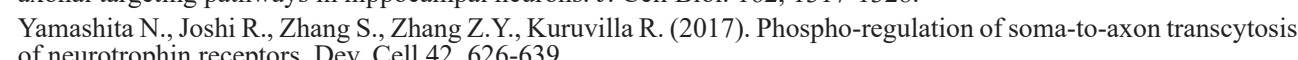

\section{SUPPLEMENTAL INFORMATION}

Figure S1. Additional controls for SNAP-TrkB and RUSH-TrkB expression and trafficking dynamics - related to Figure 1

(A,B) Neurons were transfected with SNAP-myc-TrkB and cytosolic tagBFP2. (A) After $24 \mathrm{~h}$, neurons were surface labeled with anti-myc then fixed and permeabilized for whole-cell myc staining. (B) To measure internalization,
neurons were pulsed labeled with surface myc antibody then incubated with BDNF or control media for 30 min followed by fixation and incubation secondary antibodies before and after permeabilization to capture surface and internalized SNAP-myc-TrkB, respectively. Images are confocal slices based on 2 independent experiments. (C) Ratio of internalized to surface SNAP-myc-TrkB was measured from cells stained as in (B). Data are based on 1 $\& 10$ cells based on 2 independent experiments. ${ }^{*}=$ Student's t-test value p-value $<0.05$. (D) Neurons were plated in microfluidic chambers to assay proximal to distal transcytosis of SNAP-TrkB as presented in Fig IF,I. Surface labeling of SNAP-TrkB was carried in the proximal compartment and BDNF was added to the distal compartmen for $1-2 \mathrm{~h}$ prior to live imaging of axons crossing from the proximal to distal compartments. Segments of the proxima axon and along the axon were captured at $1 \mathrm{~s} /$ frame for 5 min. Representative kymographs depict anterograde (white arrowheads) and retrograde (black arrowheads) transport of SNAP-TrkB carriers. Images are based on 3 independent biotin or control of RUSH-SNAP-TrkB in the plasma membrane. (E) Representative max-projection images of soma $z$-stack. (F) Mean SNAPTrkB intensity in the outline of max-projection image were measured and normalized to cytosolic mCherry signal. Plot lines depict are based on 2 independent experiments on $37 \& 32$ neurons in control and +biotin conditions, respectively. ***Student's t-test p-value $<0.001$. (G) HEK cells expressing TrkB-GFP, RUSH-TrkB-GFP and GFP only as negative control were treated with biotin for $120 \mathrm{~min}$, followed by 30 min incubation with BDNF then lysed and processed for WB analysis of ERK1/2 activation using antibodies against phosphorylated ERK1 (pThr202) \& ERK2 (pTyr204) and total ERK1/2. (H) Ratios of phosphoERK1/2 to total ERK1/2 bands from 3 independen experiments. * $\alpha$ Student's t-lest $p<0.05 \& \mathrm{p}<0.01$, respectively. (I) Neurons were treated with either contro mM130 (Golgi). Images are based of 3 independent experiments. Scale bars: $5 \mu \mathrm{m}$. Data in graphs are mean \pm S.E.M.
GM.
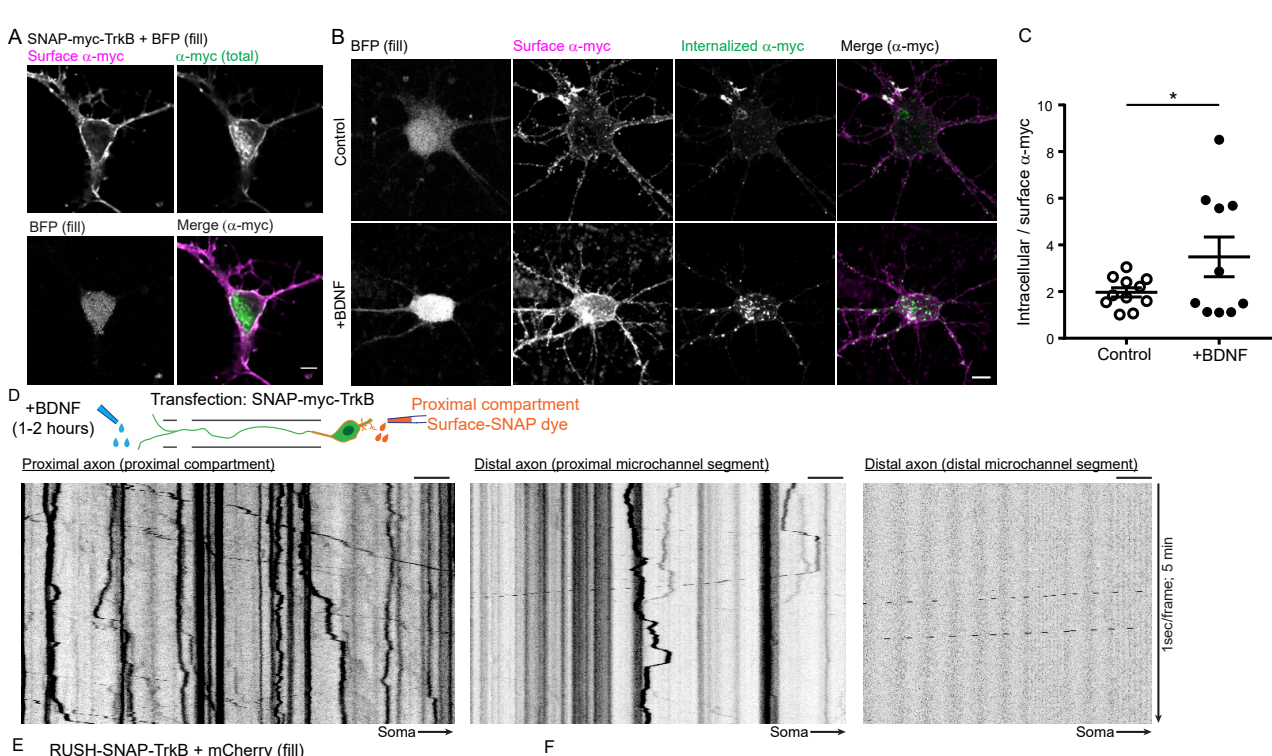

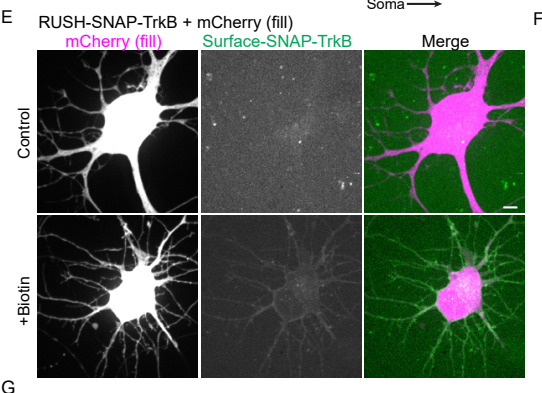

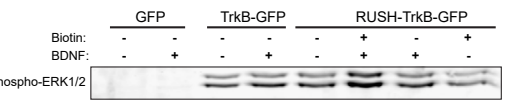

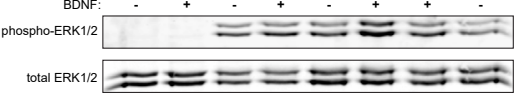

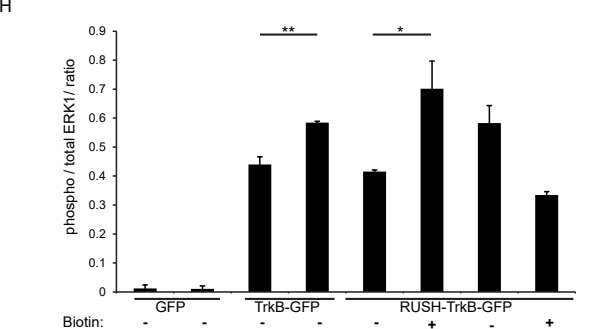

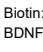
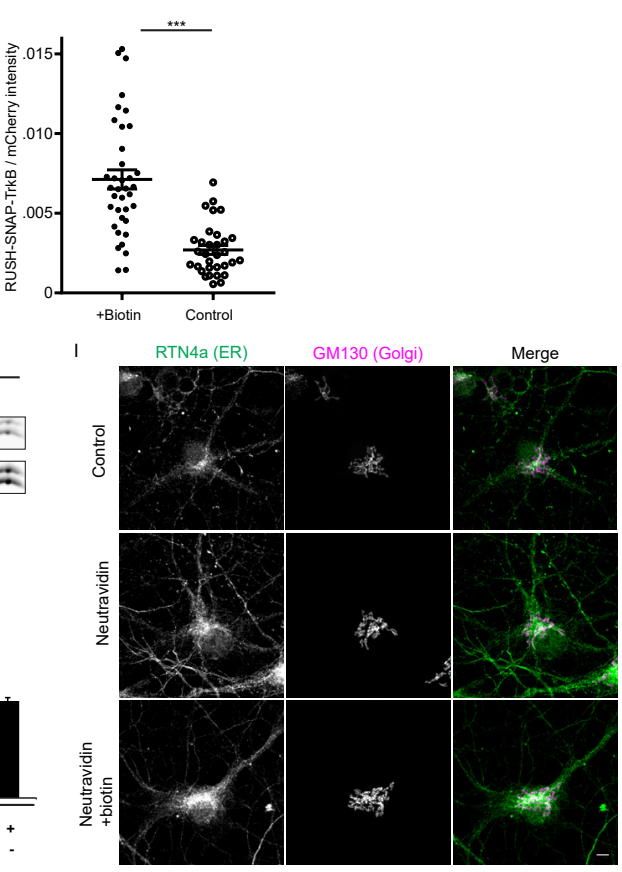

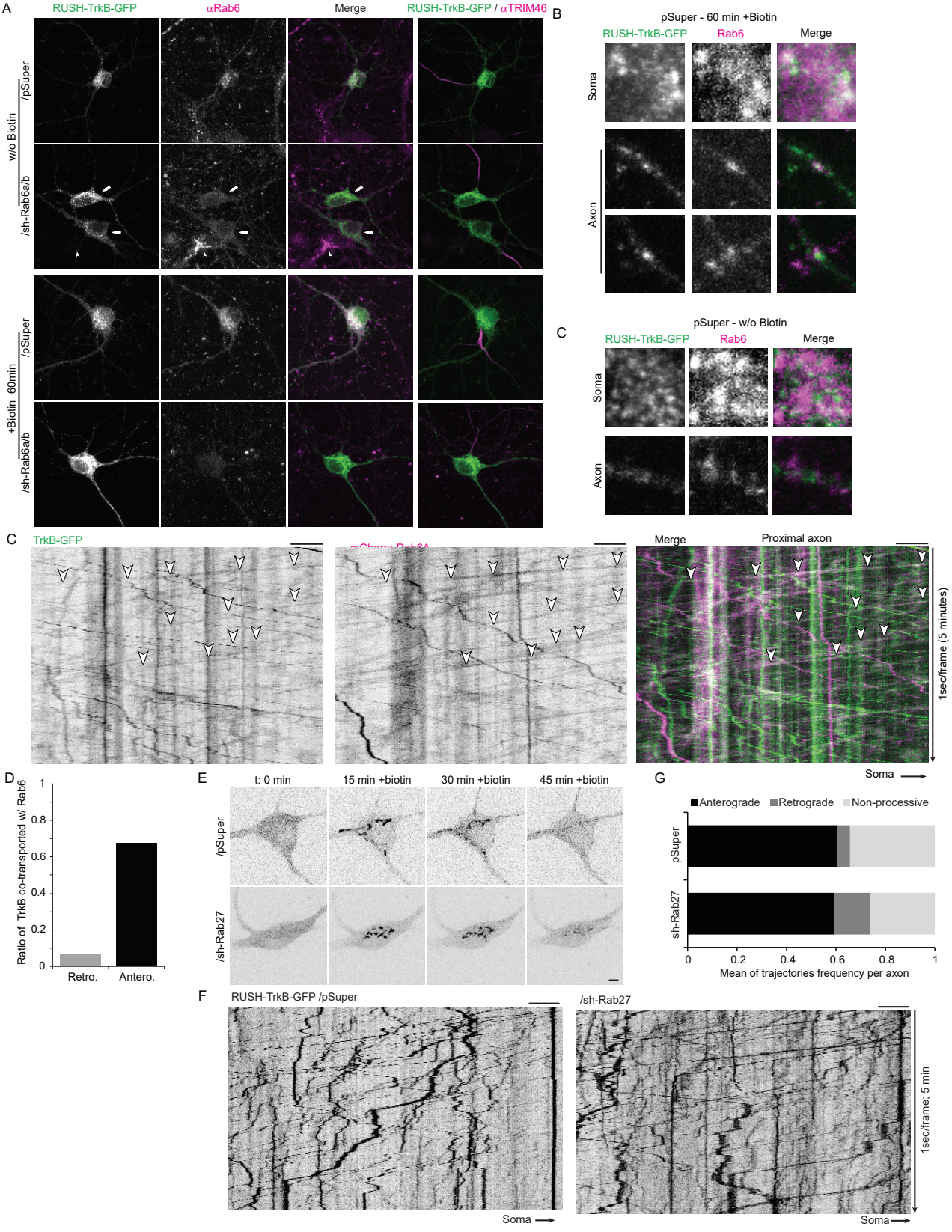

Figure S2. Additional validation experiments on axon transported TrkB interaction with Rab6 and Rab27 related to Figure 5

(A) Neurons were co-transfected with RUSH-TrkB-GFP and shRNA against Rab6a \& Rab6b or pSuper control vector and trated with control or biotin supplemented media for $60 \mathrm{~min}$ followed by fixation and immunostained for Rab6 stainin are maked by arrows. Arrowhed marks a nis oll pering of soma and axon ares byowing coregion and in puncta along the axon in biotin and untreated neurons expressing RUSH-TrkB-GFP and pSuper control. (C) Proximal axon segments of neurons co-expressing TrkB-GFP and mRFP-Rab6A were liveimaged for 5 min at 1 frame/sec. Arrowheads mark trajectories of co-transported TrkB and Rab6-positive carriers. (D) Co-localization ratio of TrkB+Rab6 to total TrkB trajectories analyzed in the anterograde and retrograde direction. Data is pooled from 109 retrograde and 131 anterograde trajectories, from 12 separate axons in 3 independent experiments. (E-G) Neurons were co-transfected with pSuper control or shRNA constructs against Rab27A \& Rab27B and RUSH-TrkBGFP. Live imaging was carried 2-3 days after transfection. (E) Soma was imaged in the course of $45 \mathrm{~min}$ following the addition of biotin at $1 \mathrm{~min} / \mathrm{fame}$. (, G) Proximal axons were inaged at a time window or $45-90 \mathrm{~min}$ after bioti addition at a frame rate of $1 \mathrm{sec} /$ frame for 5 mins. Kymograph trajectories were manually traced and quantified to based on 12 (pSuper) \& 11 (shRab27) axons from 3 independent experiments. Scale bars: $5 \mu \mathrm{m}$ (A), $2 \mu \mathrm{m}$ (BC). 


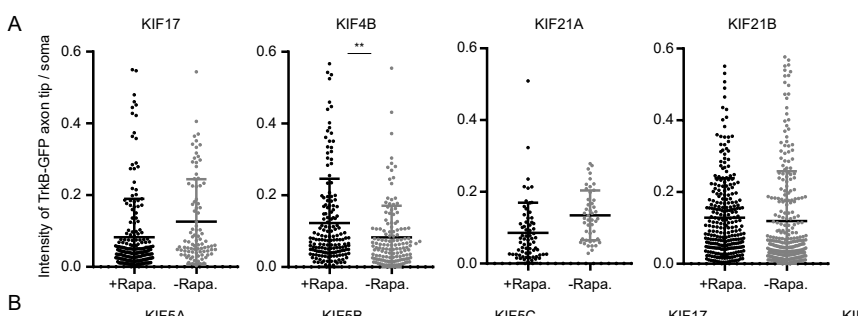

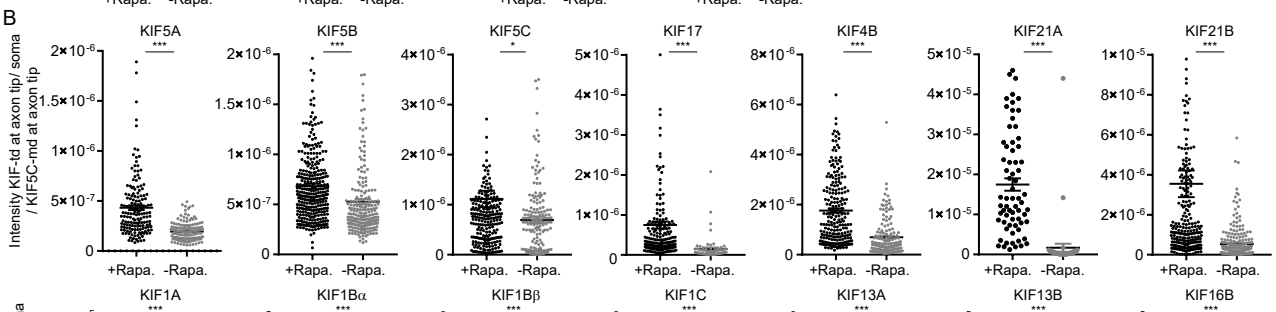

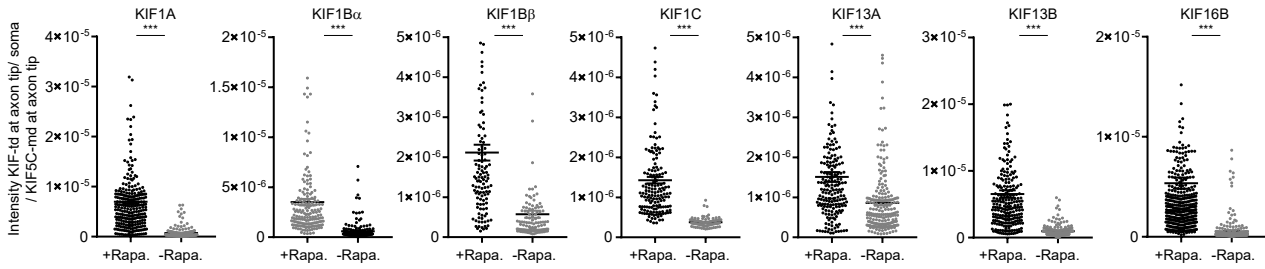

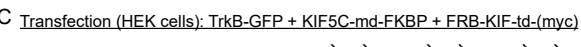

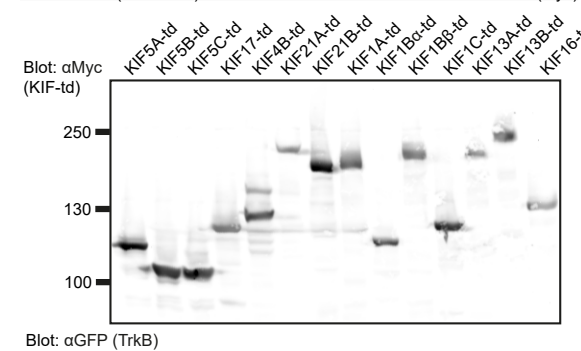

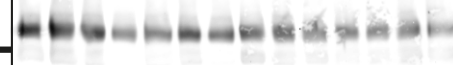

Figure S3. Additional control data of split kinesin assay - related to Figure 6

plit kinesin assay was carried in neurons as described in Fig 4B (A) TrkB-GFP axon tip enrichment in neurons tested individual axon tips normalized to the mean intensity in its corresponding soma. Lines depict mean and S. D. ** * Two sided Student's t-test, $p<0.01$. Data points were collected from the following number of axon tip: KIF17: 197897 , KIF4B: $161 \& 139$, KIF21A· 67\&46, KIF21B: $267 \& 276$ for +Rapa \& -Rapa conditions, respectively (B) Validation of kinesins tail-domain recruitment to axon tips in split kinesin assays presented in (A) and in Fig 4E,F. Axon tips and their respective soma were imaged and intensity of KIF-td and KIF5C-md was measured. KIF-td signal at the axon tip was divided by KIF-td signal in the soma and KIF5C-md signal in the tip to calculate the relative enrichment of KIF-td at the tip in rapalog vs. control conditions. Lines depict mean and S.D. * $\& * * *$ Two-sided Student's t-tes $p<0.05 \& p<0.001$. Data points were collected from the following number of axon tip: KIF5A:160 \& 147, KIF5B 323\& 219, KIF5C: 241 \& 217, KIF17: 199 \& 70, KIF4B: 228\&140, KIF21A: 68\&46, KIF21B. 267\&263, KIFIA: 277\&213, KIF1Ba: 173\&168, KIF1Bb: 120\&11, KIFIC: $160 \& 86$, KIF13A: $189 \& 231$, KIF13B: 237\&230, KIF16B: $389 \& 297$ for +Rapa \& - Rapa conditions, respectively. (C) HEK cells were transfected with SKA constructs, lysed respective expression between different KIF-td constructs. Blot shown is representative of 4 independent experiments.
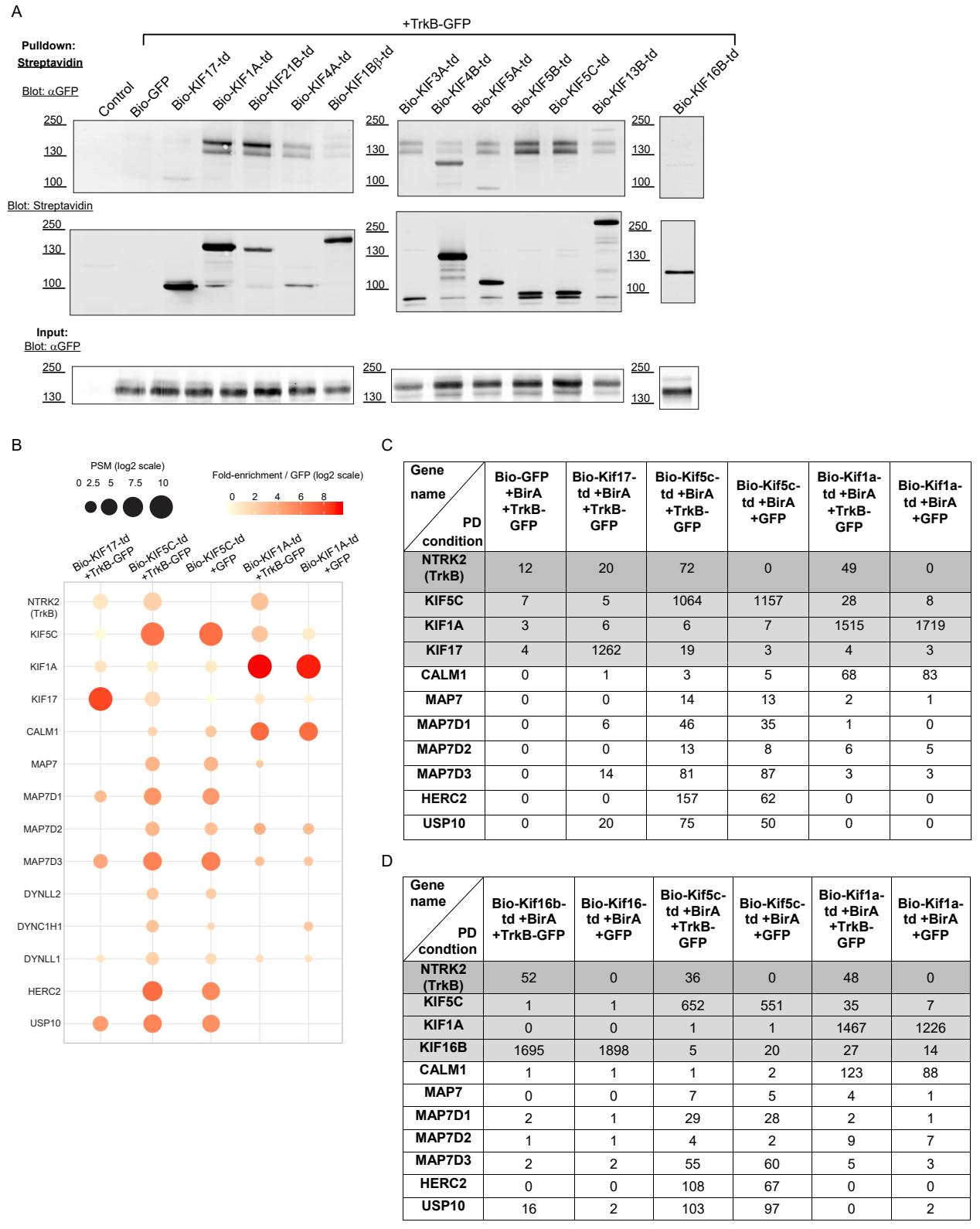

(A)

(A) HEK cells co-expressing TrkB-GFP, BirA and specified Bio-tagged kinesin-tail domain constructs (BioKIF) or TrkB-GF, BirA and bio-GrY as negative control were lysed and processed for a Streptavidin basepulldown. The pulddown elution and total lysate fraction were probed with an antibody against GrP to detect of HEK cells expressing the constructs specified in the x-axis were collected as described in (A). Sann eluates processed for MS proteomic analysis. The bubble plot represents the protein abundance (PSM), depicted bubble size and enrichment over TrkB-GFP+GFP (negative control condition) depicted as bubble color (C,D) of PSM values of selected proteins detected in Bio-Kif-td pulldown (PD) mass-spectrometry analyses \#1 \& \#2. 


\section{General discussion}

Jessica J.A. Hummel ${ }^{1}$

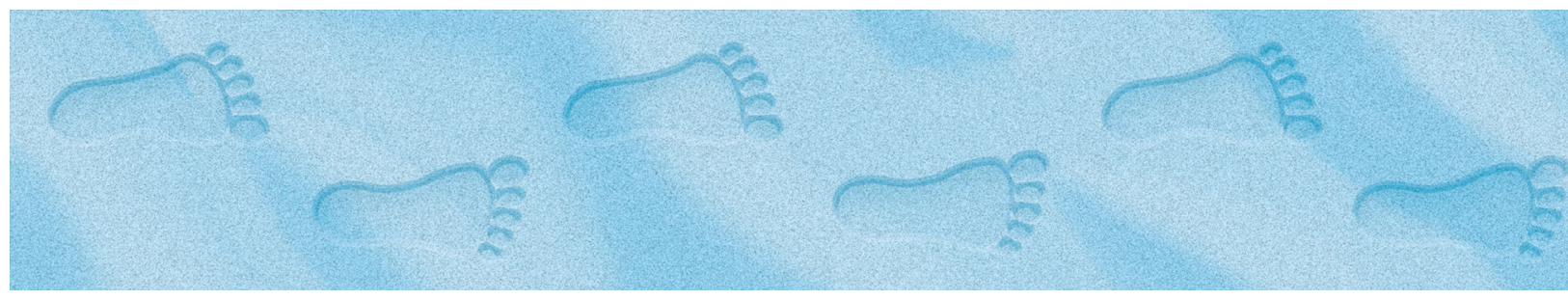

${ }^{1}$ Cell Biology, Neurobiology and Biophysics, Department of Biology, Faculty of Science, Utrecht University, 3584 CH Utrecht, the Netherlands. 
Neurons are highly polarized cells that rely on intracellular cargo transport to maintain their specialized architecture and function. Despite much research into the cargo trafficking machinery, still relatively little is known about how kinesin, dynein, and myosin motors are regulated to ensure proper distribution of cargo with high spatiotemporal control. This thesis the main findings and provide directions for future research.

\section{Engineered motors to study cargo trafficking}

Over the years engineered motors have been used to study cargo trafficking in cells. An overview of assays using engineered motors is shown in Table 1 . These will be discussed investigate the function of Bicaudal D protein in minus end directed transport of mitochondria and peroxisomes (Hoogenraad et al, 2003). This system was further developed into the rapalog-inducible peroxisome assay, which since then has been used to study kinesin motor unction (Kapitein et al., 2010). The peroxisome motility assay uses a kinesin motor domain (md) fused to a FKBP12-rapamycin-binding (FRB) domain and a peroxisome targeting signal (PEX) that is fused to a FKBP domain. Rapalog induces the chemical interaction between the FRB and FKBP domains, thereby linking the kinesin motor to peroxisomes, which results in transport of peroxisomes out of the cell center into the periphery (Fig 1A). Over time the assay has been used extensively for example to identify all transporting or to study motility of kinesin-4 motors (Lipka et al., 2016; Yue et al., 2018). In Chapter 2 we have combined structural and functional data by using the peroxisome motility assay and cryo-electron microscopy data to elucidate the interaction mode between kinesin-binding protein (KBP) and the motors KIF15 and KIF1A. The peroxisome assay provides a robust system to study kinesin motor function and has several advantages. First, its use in COS7 cells makes it a low-cost, easily available method to study kinesin motor regulation. Initial studies have used live-cell imaging methods as main analysis method, making the experiments more challenging and time-consuming. However, in Chapter 2, we show that addition of rapalog directly after transfection of cells leads to a robust peroxisome re-localization that allows analysis in fixed cells. This enables quick initial screening of the results by eye and makes further quantification easier. A disadvantage of the peroxisome motility assay is that once activated, organelle transport cannot be reversed. To overcome this, an inducible system based on an optogenetic strategy has been developed. This light-inducible peroxisome assay uses the TULIP system, in which a photosensitive LOV domain from A. sativa phototropin is fused to PEX and a kinesin motor domain is fused to the engineered PDZ domain (ePDZ) Illumination with blue light results in an interaction between the LOV and ePDZ domain, thereby activating transport which is switched off when returning to a dark state (Fig 1A) Van Bergeijk et al., 2015). This method enables high spatiotemporal control of peroxisome translocation. However, its dependence on specific equipment and expertise makes such strategy less general accessible.

The main use of the peroxisome motility assay is to study kinesin motor function and variations of the peroxisome motility assay have been designed and used to study other aspect of the cargo trafficking machinery. For example, a different chemically inducible system, named reversible association with motor proteins (RAMP), was based on the SBP-streptavidin interaction. In this system an organelle of interest is fused to streptavidin binding protein (SBP) and either the KIFCl or KIF5B motor domain is fused to streptavidin. In the initial state, SBP interacts with streptavidin and therefore cargo is mis-localized either to the cell periphery by the original protein localization. This allows synchronizatio of the movement of an organelle from the end of a microtubule (MT) and enables studying
the kinetics of organelle movement by endogenous motors (Fig 1B) (Guardia et al., 2019).
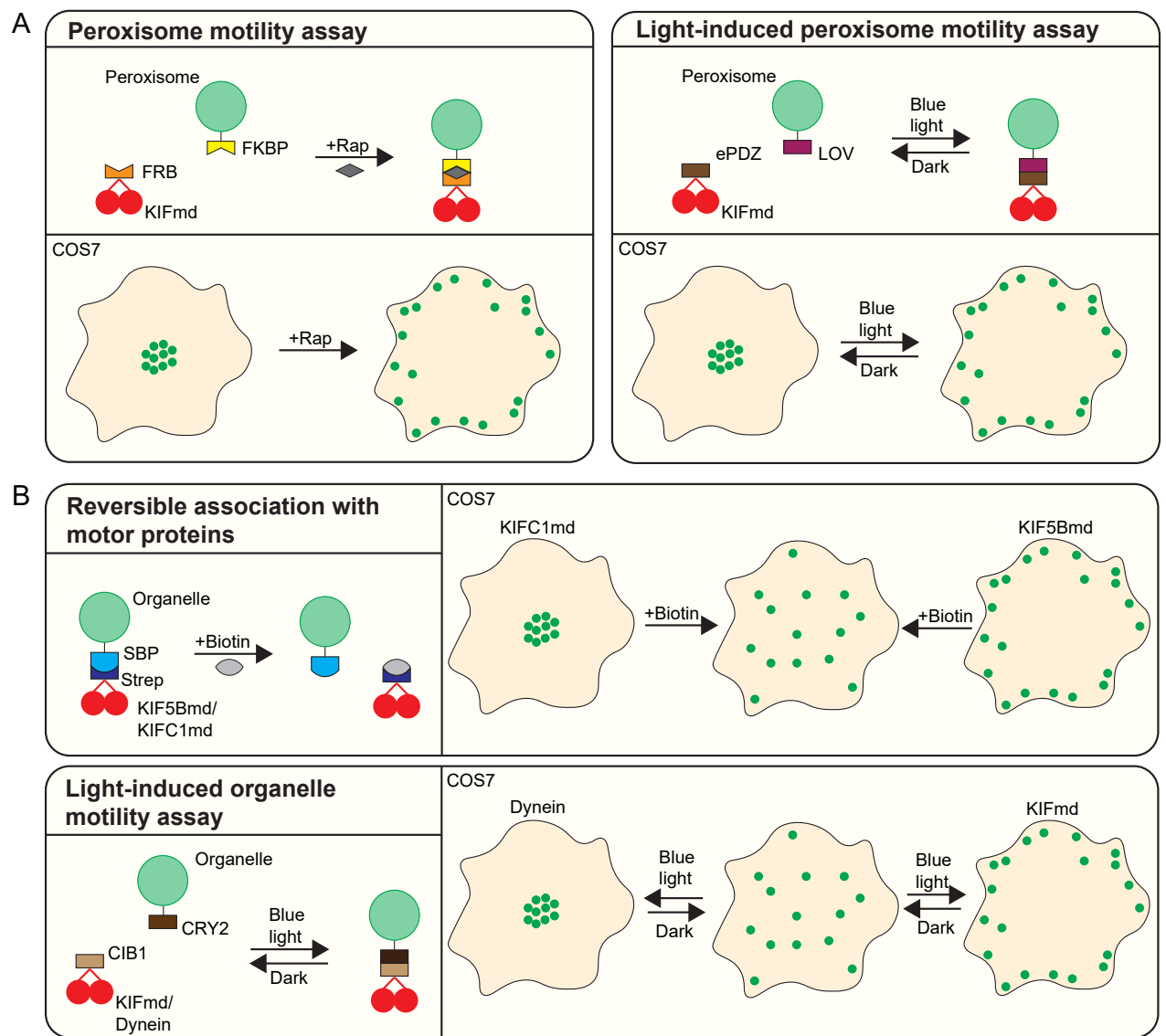

ssays in $\operatorname{Cos} 7$ cells

(A) Overview of inducible transport assays used to study kinesin motor function. The peroxisome motility assay consists of a kinesin motor domain fused to FRB and PEX fused to FKBP. Rapalog addition leads to binding o light-induced peroxisome motility assay uses a kinesin motor domain fused to $\mathrm{PDZ}$ and PEX fused to a LOV dim. Blue light illumination results in translocation of peroxisomes into the periphery, which is reversed when returning to a dark state (right). (B) Overview of inducible transport assays used to study organelle trafficking and dynamics. The reversible association with motor proteins (RAMP) system uses the KIF5C or KIFC1 motor domain fused to Strep and an organelle fused to SBP. Depending on the motor domain the organelle is either transported to the cell center (KIFC1) or cell periphery (KIF5C). Biotin addition disrupts the motor-organelle interaction, allowing organelles to restore their normal distribution (top). A comparable light-induced organelle motility assay uses either a KIF or dynein motor domain fused to $\mathrm{CIBI}$ and an organelle fused to CRY2. Bue light illumination results in transport of the organelle into the cell center (dynein) or cell periphery (KIF), which is reversed when returning to a dark state (bottom).

In theory, this can be reversed by wash-out of biotin, however, in practice this has proven to be challenging. A different method to reversibly regulate organelle transport was based on the optogenetic CRY system. Here, an organelle of interest is fused to Arabidopsis thaliana cryptochrome 2 (CRY2) and either a kinesin motor domain or dynein is fused to CIB1. The interaction between CRY 2 and CIBl is controlled by exposure to blue light, thereby generating a system in which cargo transport can be regulated with high spatiotemporal control (Fig 1B)

The assays described so far have mainly been used in COS7 cells and therefore one

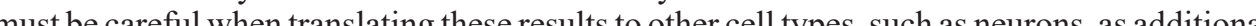
regulating factors that might lack in COS7 cells, could be involved in cargo trafficking 

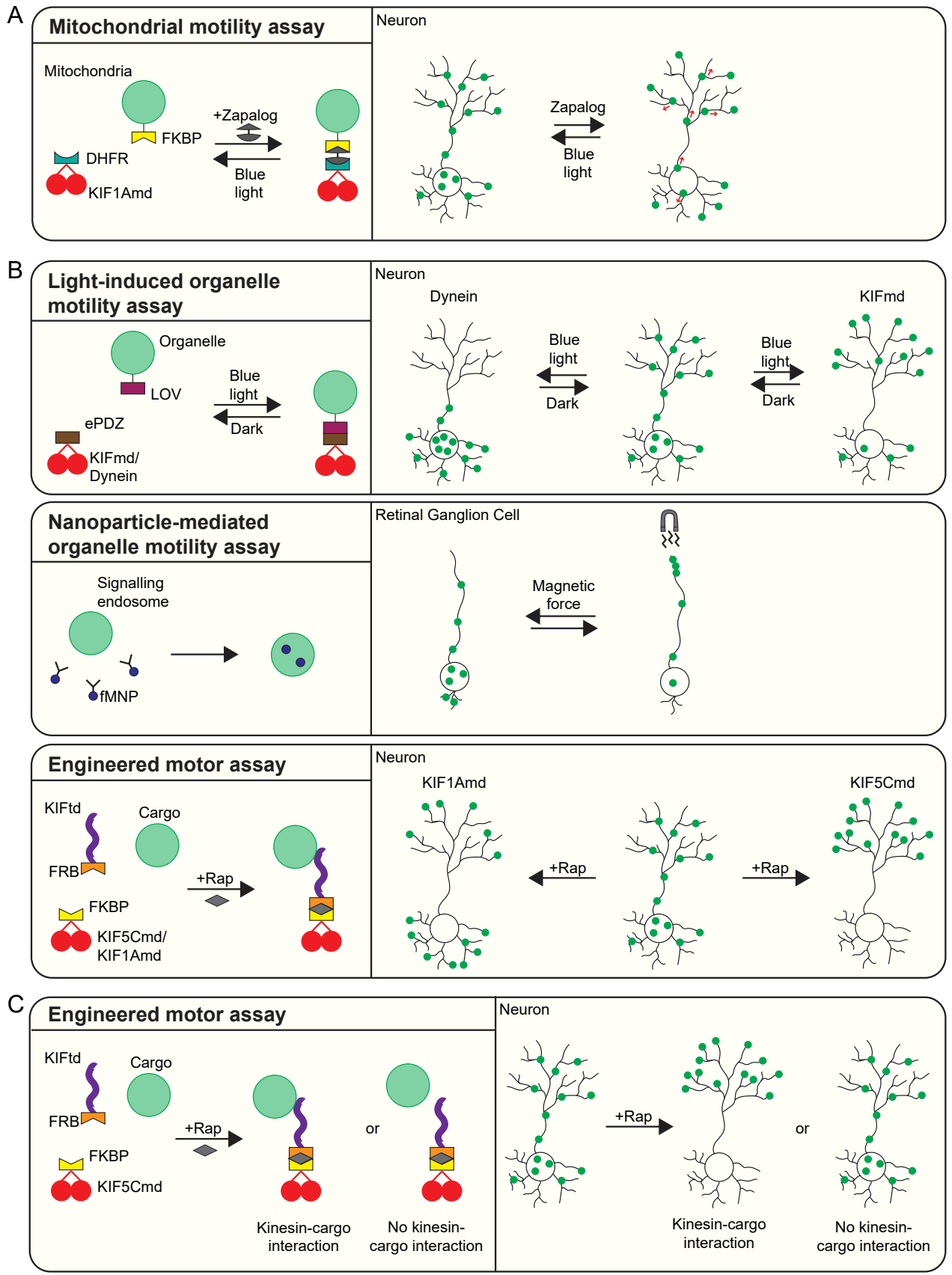

Figure 2. Overview of inducible transport assays in neurons

(A) Overview of inducible transport assays used to study organelle trafficking and dynamics. A mitochondrial motility assay uses the KIF1A motor domain fused to DHFR and mitochondrial protein TOM20 fused to FKBP. Zapalog addition leads to binding of mitochondria to the motor and results in translocation of mitochondria into the axon and dendrites. Blue light illumination leads to photolysis of zapalog and restores mitochondrial motility. (B) Overview of inducible transport assays used to manipulate polarized cargo trafficking. A light-induced peroxisome
motility assay uses either a kinesin motor domain or dynein fused to ePDZ and an organelle fused to a LOV domain.
Blue light illumination results in translocation of the organelle into the somatodendritic region (dynein) or the axon (KIFmd), which is reversed when returning to a dark state (top). Another system uses signaling endosomes that are functionalized with fMNP antibodies in retinal ganglion cells. Here, application of a magnetic force is used to direct endosomal movement (midde). The engineered motor assay uses either the KIFIA or KIF5C cargo transport into axonal and dendritic tips (KIF1Amd) or only into axonal tips (KIF5Cmd) (bottom). (C) Overview of inducible transport assays used to study kinesin-cargo interactions. The engineered motor assay uses the KIF5C motor domain fused to FKBP, a kinesin tail domain fused to FRB and a cargo. Rapalog addition induces cargo transport into the axon in case of an interaction between the kinesin tail domain and cargo.

It has been shown that the peroxisome assay can be used in neurons to study kinesin motility and RAMP also has been used in neurons to study polarized transport of neuronal lysosomes (Guardia et al., 2019; Lipka et al., 2016). Another method to study cargo distribution and dynamics in neurons uses the light-sensitive chemical reagent zapalog. Here, the KIF1A motor domain is fused to a DHFR domain and the mitochondrial protein TOM20 is fused to FKBP. Zapalog induces dimerization the DHFR and FKBP domains, thereby activating mitochondrial transport out of the cell soma by KIF1A. Illumination with blue light induces photolysis of zapalog and restores mitochondrial motility (Fig 2A). After local illumination, uncleaved zapalog from the surrounding area can outcompete lysed zapalog molecules, thereby providing a reversible system to study organelle dynamics (Gutnick et al., 2019) Other methods to study the cargo trafficking machinery have focused on manipulating polarized cargo transport in neurons, which has provided new insights in the importance of specific cargo trafficking for neuronal development. For example, an optogenetic strategy was used to study the effect of endosome mislocalization on growth cone dynamics. Using the TULIP system with the endosomal RAB11 protein fused to a LOV domain and either kinesin or dynein fused to ePDZ cargo transport could be directed either in or out of the growth cone (Fig 2B) and it was found that kinesin-driven endosomal enrichment in axonal growth cones is important for axon growth (Van Bergeijk et al., 2015). Another method used superparamagnetic nanoparticles (fMNPs) functionalized with TrkB antibodies that are endocytosed into signaling endosomes. The localization of these fMNP-signaling endosomes can be manipulated using magnetic forces, thereby controlling organelle positioning (Fig 2B) and it was shown that the localization of these signaling endosomes affects growth cone behavior and neurite outgrowth (Steketee et al., 2011). The optogenetic and magnetic cargo motility assays provide elegant methods to study the effects of cargo mis-localization on neuronal development. However, their dependence on specific techniques and expertise makes them less general available. In addition, the magnetic assay has so far merely been used in retinal ganglion cells and it might be difficult to apply this method in other cell types for example in cultured hippocampal neurons. In Chapter 3 we describe the developmen of an engineered motor platform, that provides a method to manipulate cargo trafficking in neurons. The platform is based on split kinesins, where a kinesin motor domain is fused to FKBP and a kinesin tail domain to FRB. Addition of rapalog results in formation of a fully functional motor that will translocate the cargo that interacts with the tail domain. Using either the KIF5C or KIF1A motor domain, cargo can be directed into the axon or into both the axon and dendrites, respectively (Fig 2B). Thereby, the platform enables the manipulation of polarized cargo trafficking. Furthermore, we optimized analysis methods in fixed cells, thereby overcoming the necessity for challenging live-imaging experiments.

Our engineered motor platform can also be used to study kinesin-cargo interactions. For this purpose, the KIF5Cmd fused to FKBP is used and a library of kinesin tail domain fused to FRB was generated. When these constructs are co-expressed with a fluorescently labeled cargo, addition of rapalog will result in re-localization of cargo into the axon if there is an interaction between the cargo and the kinesin tail domain (Fig 2C). This enables rapid screening and identification of kinesin-cargo interactions. In Chapter 4 several applications of this assay are described, including identification of minimum binding domains, investigation of kinesin regulatory mechanisms and study of cargo adaptors. 
To conclude, different assays using inducible transport systems have been developed to study specific aspects of the cargo trafficking machinery. It should be noted that these are all artificial assays and results obtained from these studies should be further investigated using other techniques to validate them. This is illustrated in Chapter 2, which describes the elucidation of interaction modes between KBP and KIF15 or KIF1A by cryo-electron microscopy. These interactions modes are further mapped using the peroxisome motility assay with different KBP mutations, thereby providing an elegant example of how the use of engineered motors combined with techniques from the field of structural biology can result in a detailed map of a kinesin-protein interaction mode. In Chapter 4 and 5, results from the engineered motor platform are combined with other biochemical techniques to elucidate the regulatory mechanism of KIF1A cargo trafficking and the mechanism of TrkB transport by KIF1A and KIF5. Here, results obtained using engineered motors are further studied using other experiments, thereby validating the motor assay. For example, in Chapter 4 different truncated KIF1A fragments were subjected to the engineered motor assay, which then showed that the CC3 and PH domains of KIF1A are required for the cargo interaction. This was further validated using KIF1A knock down and rescue experiments with differen KIF1A deletion constructs. Chapter 5 identified KIF1A and KIF5 as kinesins involved in the interaction with TrkB vesicles. These interactions were further validated using pull down assays. Altogether, these examples show that engineered motors combined with additional experiments provide a powerful tool to study cargo trafficking in neuronal cells.

\section{Model for KIF1A motor regulation}

Much of the work described in this thesis has focused on elucidating the regulation of the KIF1A motor. Here, by combining our data and results from other groups, we propose a model for the regulation of neuronal KIF1A cargo trafficking. Monomeric KIF1A consist of a N-terminal motor domain followed by three coiled-coil domains with a FHA domain between $\mathrm{CC} 1$ and $\mathrm{CC} 2$, and a $\mathrm{C}$-terminal PH domain. Dimerization of KIF1A is mediated by the CC2 domain (Fig 3A). In neurons, KIF1A exists in a dimeric, autoinhibited form. In this form, the KIFIA tail domain is folded back and interacts with the $\mathrm{CCl}$ domain, thereby inhibiting the motor domain. Enhanced neuronal activity and subsequent increases in $\mathrm{Ca}^{2}$ levels lo inhibition and rendering KIF1A in an activated state. CaM w phosphorylates the KIF1A PH domain. The phosphorylated PH domain can interact with any of the KIF1A cargo's, thus with all three dense core vesicles (DCVs), lysosomes, and synaptic vesicles (SVs). The KIF1A-cargo interaction is further stabilized by specific adaptors, where Arl8A acts as an adaptor for DCVs and lysosomes, and MADD as an adaptor for SVs (Fig $3 \mathrm{~B})$. Activity and phosphorylation dependent mechanisms have been described to regulate cargo transport in other contexts. For example, it has been shown that in C. elegans neuronal activity and CaMKII regulate AMPA receptor trafficking by KIF5 (Hoerndli et al., 2015). It was also found that CaMKII dependent phosphorylation of KIF17 disrupts the interaction with the cargo adaptor Mint1, resulting in release of the cargo (Guillaud et al., 2008). It is interesting that CaMKII dependent kinesin-phosphorylation has different regulatory functions for different kinesins, and this might provide a method to co-ordinate transport between a variety of motors.

Once a stable KIF1A-cargo state is achieved, KIF1A is ready to transport its cargo along MTs. However, in areas with high KBP levels, KBP will inhibit the KIF1A motor thereby blocking cargo transport. At low cellular KBP levels KIF1A will transport its cargo along MTs until it reaches an environment with high levels of liprin- $\alpha$ and TANC2. Here, liprin- $\alpha$ and TANC2 will compete with the adaptor for binding to the KIF1A CC3 domain, thereby weakening the KIF1A-cargo interaction, which results in release of the cargo in the designated area (Fig 3C). Previously, it has been suggested in release of the cargo in the designated area (Fig 3C). Previously, it has been suggested
that liprin- $\alpha$ and TANC2 act as signposts in dendritic spines (Stucchi et al., 2018).
Liprin- $\alpha$ and TANC2 are also found enriched at presynaptic axonal sites (Han et al., 2010; Spangler et al., 2013) and this might be a general mechanism for KIF1A-cargo release in neurons. As TANC2 is much less abundant in axon terminals than in dendritic spines (Han et 1. 2010), it could be that other complexes have similar signpost functions. For example, it was found that neurotransmitter release from SVs is regulated by a MALS-liprin- $\alpha$ presynaptic complex (Olsen et al., 2005). Furthermore, a role for synapsin has been suggested in DCV capture and neuropeptide release at presynapses (Yu et al., 2021), suggesting that different signposts might regulate the capture of specific cargo at specific synaptic sites.

Cargo transport by KIF1A requires different layers of regulation, including neurona activity, phosphorylation and binding of adaptors and co-factors. Often, multiple motors are attached to one cargo vesicle and additional layers of regulation facilitate the co-operation between different motors in cargo trafficking. For example, specific MAPs and different MT affinities can determine which motor predominantly transports. It was shown that DCVs are transported into the axon by coordinated movement of KIF1 and KIF5, which is regulated by a pre-axonal MAP2 filter. In this pre-axonal region, MAP2 inhibits KIF5 motility, thereby enabling fast cargo transport into the axon by KIF1. Further down the axon, in absence of MAP2, KIF5 takes over and facilitates slow axonal transport into the growth cones (Gumy MAP2, KIF5 takes over and facilitates slow axonal transport into the growth cones (Gumy et al., 2017). In addition, it was found that transport of Rab6-containing secretory vesicles is movement of motors on a cargo vesicle by activating KIF5B and inhibiting KIF13B. This results in slow transport by KIF5B from the cell center, where older MTs are decorated with MAP7, which then switches to fast transport by KIF13B towards the cell periphery, where newly formed, dynamic MTs are devoid of MAP7 (Serra-Marques et al., 2020). In chapter 5 , we describe that trafficking of TrkB vesicles from the secretory compartment into and along the axon is mediated by coordinated movement of the KIF1A and KIF5C motor. Based on these findings one could speculate about a mechanism for coordinated axonal trafficking of cargo by KIF1A and KIF5. Initially, the KIF5 motor dominates vesicle movement from the secretory compartment towards the axon. In the pre-axonal zone, MAP2 inhibits KIF5 motor activity and fast transport of the cargo into the axon is facilitated by KIF1A. Once in the axon, MTs decorated with MAP7 inhibit KIF1A motility and activate slow transport by the KIF5 motor along the axon. When the cargo moves towards the growth cone and approaches dynamic MT ends that lack. MAP7, KIF5 activity decreases and KIF1A further transports the cargo towards its destintion in the KIF5 activity d KIF5 transports only into the axon, a A different mechan dendrites. It has been shown that multiple kinesins of the same family can work together to facilitate transport and it might be that within dendrites multiple KIF1A motors attach to one cargo vesicle to mediate processive movement (Ando et al., 2014; Beeg et al., 2008; Furuta et al., 2017; Gross et al., 2007). Another possibility is that in dendrites KIF1A co-operates with dynein. It has been shown that kinesin-3 and dynein work together in different model systems (Baumann et al.,2012; Tsai et al.,2010; Wedlich-Söldner et al., 2002). Future research should further elucidate the co-operative behavior of KIF1A and dynein in hippocampal neurons.

\section{Future applications of engineered motors}

The engineered motor platform described in Chapter 3 provides a robust tool to study kinesin-cargo interactions and to manipulate the cargo trafficking machinery in polarized neurons. Throughout this thesis we show several applications of this platform. First, we show that the platform can be used to identify all kinesins that interact with a specific cargo, and we investigated the interaction of different kinesins with DCVs, TfR and TrkB Chaptens 3 and 5). We also diction of differ (Chorges for KIF1A (Chapters 3 and 4). Furthermore, the engineered motor platform can be used to
identify minimum binding domains or mutations that are crucial for the cargo interactions, 

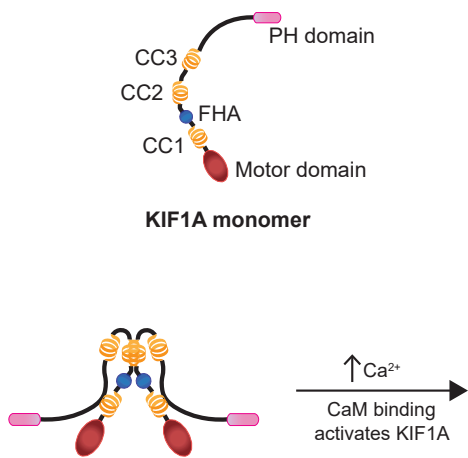

Autoinhibited KIF1A

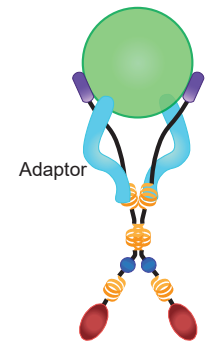

Stabilized KIF1A-Cargo

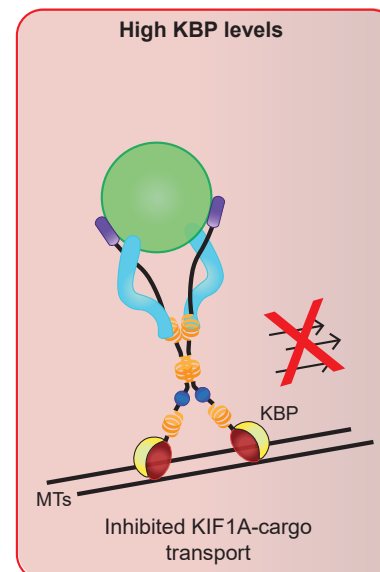

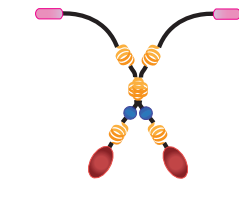

KIF1A dimer

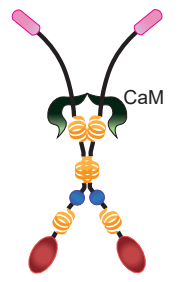

Activated KIF1A
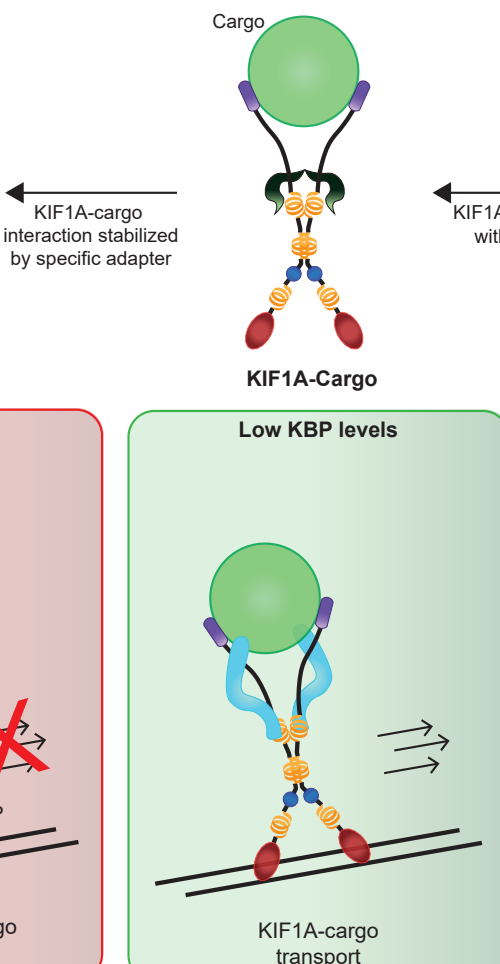

KIF1A-cargo
transport
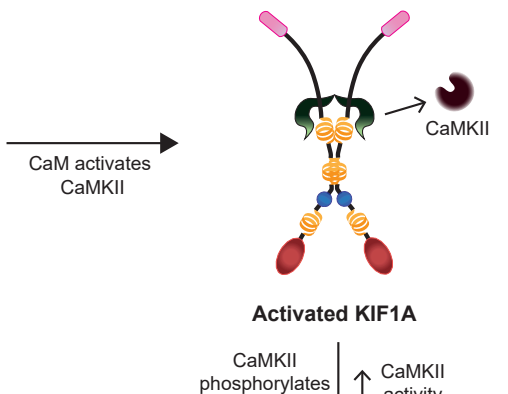

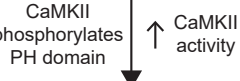

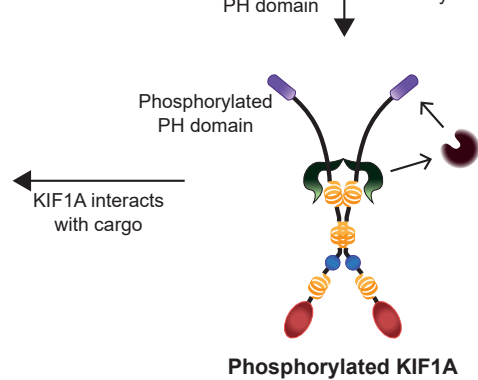

Phosphorylated KIF1A

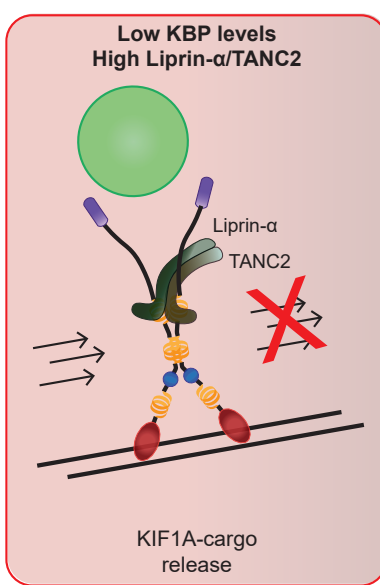

Figure 3. Model for KIF1A-cargo transport

(A) Schematic overview of monomeric and dimeric KIF1A. (B) Model for regulation of the KIF1A-cargo interaction to the KIF1A stalk domain and relieves the autoinhibition. At the same time CaM activates CaMKII, which then phosphorylates the KIF1A PH domain. The phosphorylated PH domain can interact with the cargo vesicle and this initial interaction is further stabilized by specific adaptors. (C) Model for regulation of KIF1A-cargo transport. The stabilized KIF1A-cargo complex is ready to transport cargo along MTs. However, in cellular compartments with high KBP levels, the motor domain is inhibited by KBP which impairs cargo transport (left). In absence of KBP, KIF1A will transport its cargo along MTs (middle) until it reaches an area with high liprin- $\alpha$ and TANC2 levels. Here, liprin- $\alpha$ and TANC2 will compete with the adaptor for binding to the KIF1A CC3 domain, thereby destabilizing the kinesin-cargo interaction, resulting in release of the cargo (right). and we applied this to elucidate the binding modes of KIF16B and KIF1A (Chapters 3 and 4). Engineered motors can not only be used to identify kinesin-cargo interactions, in principle the interaction of cargo with any protein of interest can be investigated. We have used the platform to identify the interaction between different KIF1A adaptors and cargo (Chapter CaMKII-dependent phosphorylation mechanism for KIFIA (Chapter 4). As the platform is robust and easily adaptable, we foresee many more applications of our engineered motor in future research on the neuronal cargo trafficking machinery, which are discussed below. In this thesis engineered motors were used to identify kinesin-cargo interactions. In principle, the assay can be used to identify the interaction of a cargo with any motor. Therefor, the library of kinesin tail domains described in Chapter 3 could be expanded with tail domains from different myosins or components of the dynein complex, which will allow investigation of cargo interactions with myosin and dynein motors. Furthermore, the assay can be used to investigate the interaction of cargos with other proteins, such as scaffolding explored the interaction of different KIF1A adaptor with cargo using engineered motor-adaptor fusion constructs and this can further be exploite to detect interactions with other scaffolding proteins and adaptors. Additionally, one could use the platform to identify the minimum binding domains required for the cargo interaction in these proteins. For example, structural studies have provided insights into the interaction mode of kinesin-1 with its cargo JIP3 (Cockburn et al., 2018). These findings could be further validated in a neuronal context using engineered motors. It would also be of interest to generate chimeras of specific kinesin tail and adaptor domains to investigate the interplay between these proteins in cargo binding. It has been shown that KIF13B interacts with cargo via the adaptor protein centaurin- $\alpha 1$ (CENTA1). Crystal structures have elucidated the interaction mode and revealed that KIF13B interacts with CENTA1 via its FHA domain CENTA1 then interacts with PIP3 vesicles via its first PH domain or with both PIP3 and PIP2 vesicles via its second PH domain (Tong et al., 2010). Making a chimera of the KIF13B tail domain in which the FHA domain is replaced by the first or second PH domain of CENTAI could further unravel this complex interaction mode and resolve preferences for specific cargo binding. Another application of the platform would be to identify factors that inhibit the kinesin-cargo interaction. Co-expressing potential inhibitory proteins in the assay will allow the detection of proteins that negatively regulate the kinesin-cargo interaction. Thus, future research on better understanding motor-cargo interaction modes will greatly benefit from the developed engineered motor platform.

There are several mechanisms that directly or indirectly regulate cargo trafficking, such as motor phosphorylation, sensing calcium levels and tubulin modifications. In Chapter 4 we used engineered motors to identify a phosphorylation mechanism to regulate KIF1A activity. Running the motor assay in presence of KN-93, a specific kinase inhibitor, showed that the interaction between KIF1A and DCVs depends on phosphorylation by CaMKII. Phosphoregulatory mechanisms have been described to regulate the activity of severa kinesins, for example KIF17 (Guillaud et al., 2008), and by using the engineered motor platform in presence of specific kinase or phosphatase inhibitors, such mechanisms can be further elucidated. Similar approaches can be used to study other potential regulatory mechanisms, such as palmitoylation or sumoylation. Furthermore, such an approach can be used to investigate the effects of neuronal activity on motor activity. Cellular calcium levels can be manipulated using specific drug treatments, which enables studying the effects of calcium on kinesin-cargo transport. As cargo transport by several kinesins is regulated by neuronal activity (Hoerndli et al., 2015; Wang and Schwarz, 2009), these strategies will be useful in studying kinesin motor function in a neurophysiological context. 
It would also be of interest to apply engineered motors in other model systems, such as Caenorhabditis elegans, living mice, or stem cells. Using in vivo systems could provide more information on how mistargeting cargo effects the whole neuronal network. It has been shown that light-inducible activation strategies can be used in in vivo systems, thereby enabling studying the cargo transport mechanisms within the context of a developing anima (Harterink et al., 2016). As motor proteins and defects in the cargo trafficking machinery have been linked to a variety of diseases, including birth defects, neurodevelopmental disorders, and neurodegeneration (Eschbach and Dupuis, 2011; Guo et al., 2020; Kalantari and Filges, 2020), such strategies could be used to study the role of cargo transport in specific disease state. Another application would be to use engineered motors in context of specific disease related mutations. Therefor, one could express the engineered motor system in mutated C. elegans strains or use patient-derived stem cells and investigate kinesincargo interaction under these mutations. For example, in Goldberg-Shprintzen syndrome (GOSHS), KBP inactivating mutations have been described to cause various dysfunctions (Chang et al, 2019). (Chang et al, 2019). more information on whether these mutations disrupt the KBP-mitochondria interaction or the interaction of KBP with KIF1A, thereby providing more information on how certain mutations influence cargo trafficking. Furthermore, mutations in motors have been described in disease. For example, several mutations in KIF1A were found to cause KIF1A associated neurological disorder (KAND) and in Chapter 4 we identified a disease-related mutation as a critical phosphorylation site for cargo-binding, suggesting that the mutation disrupts the interaction cargo and thereby impairs transport. Many mutations that have been identified in KAND are localized in the motor domain (Boyle et al., 2021). Also, for KIF5A several motor domain mutations that cause hereditary spastic paraplegia (HSP) have been identified (Jennings et al., 2017). The engineered motor platform could be used to study the behavior of such mutated motors and unravel mechanisms of how these mutations impair cargo transport. In addition, several mutations in dynein heavy chain were found in patients suffering from a broad range of neurological diseases and these mutations have been studied in a budding yeast screening platform (Marzo et al., 2019). The engineered platform would provide a good complementation to further study the effects of these mutations on motor function and cargo interaction in a neuronal context. Overall, we foresee a wide range of applications for engineered motors in further unravelling the cargo trafficking machinery in complex cell types, such as polarized neurons.

REFERENCES

Ande, D., Matson, M.K., Xu, J., and Gopinathan, A. (2014). Cooperative protofilament switching emerges from Baumann, S., Pohlmann, T., Jungbluth, M., Brachmann, A., and Feldbrügge, M. (2012). Kinesin-3 and dynein
mediate microtubule-dependent co-transport of mrnps and endosomes. J. Cell Sci. 125, 2740-2752.

Beeg, J., Klumpp, S., Dimova, R., Gracià, R.S., Unger, E., and Lipowsky, R. (2008). Transport of beads by severa kinesin motors. Biophys. J. 94, 532-541.

Van Bergeijk, P., Adrian, M., Hoogenraad, C.C., and Kapitein, L.C. (2015). Optogenetic control of organelle

Boyle, L., Rao, L., Kaur, S., Fan, X., Mebane, C., Hamm, L., Thornton, A., Ahrendsen, J.T., Anderson, M.P. Christodoulou, J., et al. (2021). Genotype and defects in microtubule-based motility correlate with clinical severity

Chang, H.Y., Cheng, H.Y., Tsao, A.N., Liu, C., and Tsai, J.W. (2019). Multiple functions of KBP in neural . Insights into kinesin-1 activation from the crystal structure of KLC2 bound to JIP3. Structure 26, 1486-1498.

Duan, L., Che, D., Zhang, K., Ong, Q., Guo, S., and Cui, B. (2015). Optogenetic control of molecular motors and organelle distributions in cells. Chem. Biol. 22, 671-682.

Eschbach, J., and Dupuis, L. (2011). Cytoplasmic dynein in neurodegeneration. Pharmacol. Ther. 130, 348-363 Furuta, K., Furuta, A., and Toyoshima, Y.Y. (2017). Measuring collective transport by defined numbers of processive
and nonprocessive kinesin motors. PNAS 114, E2264.
Gross, S.P., Vershinin, M., and Shubeita, G.T.T. (2007). Cargo transport: Two motors are sometimes better than one.

Guardia, C.M., De Pace, R., Sen, A., Saric, A., Jarnik, M., Kolin, D.A., Kunwar, A., and Bonifacino, J.S. (2019) Reversible association with motor proteins (RAMP): A streptavidin-based method to manipulate organell

Guillaud, L., Wong, R., and Hirokawa, N. (2008). Disruption of KIF17-Mint1 interaction by CaMKII-dependen .

Gumy, L.F., Katrukha, E.A., Grigoriev, I., Jaarsma, D., Kapitein, L.C., Akhmanova, A., and Hoogenraad, C.C.
(2017). MAP2 defines a pre-axonal filtering zone to regulate KIF1- versus KIF5-dependent cargo transport in (

Guo, W., Stoklund Dittlau, K., and Van Den Bosch, L. (2020). Axonal transport defects and neurodegeneration

Gutnick, A., Banghart, M.R., West, E.R., and Schwarz, T.L. (2019). The light-sensitive dimerizer zapalog reveals Han, S., Nam, J., Li, Y., Kim, S., Cho, S.H., Cho, Y.S., Choi, S.Y., Choi, J., Han, K., Kim, Y., et al. (2010). Regulation of dendritic spines, spatial memory, and embryonic development by the TANC family of PSD-95-interacting
proteins. J. Neurosci. 30, 15102-15112.

Harterink, M., Van Bergeijk, P., Allier, C., De Haan, B., Van Den Heuvel, S., Hoogenraad, C.C., and Kapitein, L.C. (2016). Light-controlled intracellular transport in Caenorhabditis elegans. Curr. Biol. 26, R153-R154. Hoerndli, F.J., Wang, R., Mellem, J.E., Kallarackal, A., Brockie, P.J., Thacker, C., Madsen, D.M., and Maricq, A. V. Hoogenraad, C.C., Wulf, P., Schiefermeier, N., Stepanova, T., Galjart, N., Small, J.V., Grosveld, F., De Zeeuw,
C.I., and Akhmanova, A. (2003). Bicaudal D induces selective dynein-mediated microtubule minus end-directed transport. EMBO J. 22, 6004-6015.

Jennings, S., Chenevert, M., Liu, L., Mottamal, M., Wojcik, E.J., and Huckaba, T.M. (2017). Characterization of Kalantari, S., and Filges, I. (2020). "Kinesinopathies": Emerging role of the kinesin family member genes in birth defects. J. Med. Genet. 57, 797-807.

Kapitein, L.C., Schlager, M.A., Van Der Zwan, W.A., Wulf, P.S., Keijzer, N., and Hoogenraad, C.C. (2010). Probing (2143-2152.

Lipka, J., Kapitein, L.C., Jaworski, J., and Hoogenraad, C.C. (2016). Microtubule-binding protein doublecortin-like

Marzo, M.G., Griswold, J.M., Ruff, K.M., Buchmeier, R.E., Fees, C.P., and Markus, S.M. (2019). Molecular basis

Misawa, H., Burlingame, A.L., Nicoll, R.A., et al.
Cell Biol. $170,1127-1134$

Serra-Marques, A., Martin, M., Katrukha, E.A., Grigoriev, I., Peeters, C.A.E., Liu, Q., Hooikaas, P.J., Yao, Y. Solianova, V., Smal, I., et al. (2020). Concerted action of kinesins kif5b and kifl3b promotes efficient secretory

Spangler, S.A., Schmitz, S.K., Kevenaar, J.T., De Graaff, E., De Wit, H., Demmers, J., Toonen, R.F., and Hoogenraad, C.C. (2013). Liprin- $\alpha 2$ promotes the presynaptic recrit

Steketee, M.B., Moysidis, S.N., Jin, X.L., Weinstein, J.E., Pita-Thomas, W., Raju, H.B., Iqbal, S., and Goldberg, J.L.
(2011). Nanoparticle-mediated signaling endosome localization regulates growth cone motility and neurite growth. PNAS 108, 19042-19047.

Stucchi, R., Plucińska, G., Hummel, J.J.A., Zahavi, E.E., Guerra San Juan, I., Klykov, O., Scheltema, R.A., Altelaar A.F.M., and Hoogenraad, C.C. (2018). Regulation of KIF1A-driven dense core vesicle transpor. $\mathrm{Ca}^{2} \mathrm{C}$ CaM , control

Tong, Y., Tempel, W., Wang, H., Yamada, K., Shen, L., Senisterra, G.A., MacKenzie, F,., Chishti, A.H., and Park, H.W. 2010). Phosphorylation-independent dual-site binding of the FHA domain of KIF13 mediates phosphoinositid
transport via centaurin $\alpha 1$. PNAS 107, 20346-20351. Tsai, J.W., Lian, W.N., Kemal, S., Kriegstein, A.R., and Vallee, R.B. (2010). Kinesin 3 and cytoplasmic dynein
mediate interkinetic nuclear migration in neural stem cells. Nat. Neurosci. 13, 1463-1472.

Wang, X., and Schwarz, T.L. (2009). The mechanism of $\mathrm{Ca}^{2+}-$ dependent regulation of kinesin-mediated mitochondria motility. Cell 136, 163-174.

Wedlich-Söldner, R., Straube, A., Friedrich, M.W., and Steinberg, G. (2002). A balance of KIF1A-like kinesin and

Yu, S.C., Liewald, J.F., Shao, J., Costa, W.S., and Gottschalk, A. (2021). Synapsin is required for dense core vesicle chemomechanical coupling causes impaired motility of the kinesin-4 motors KIF27 and KIF7. J. Cell Biol. 217 1319-1334. 


\section{SUPPLEMENTAL INFORMATION}

Table 1. Overview of engineered motor assays

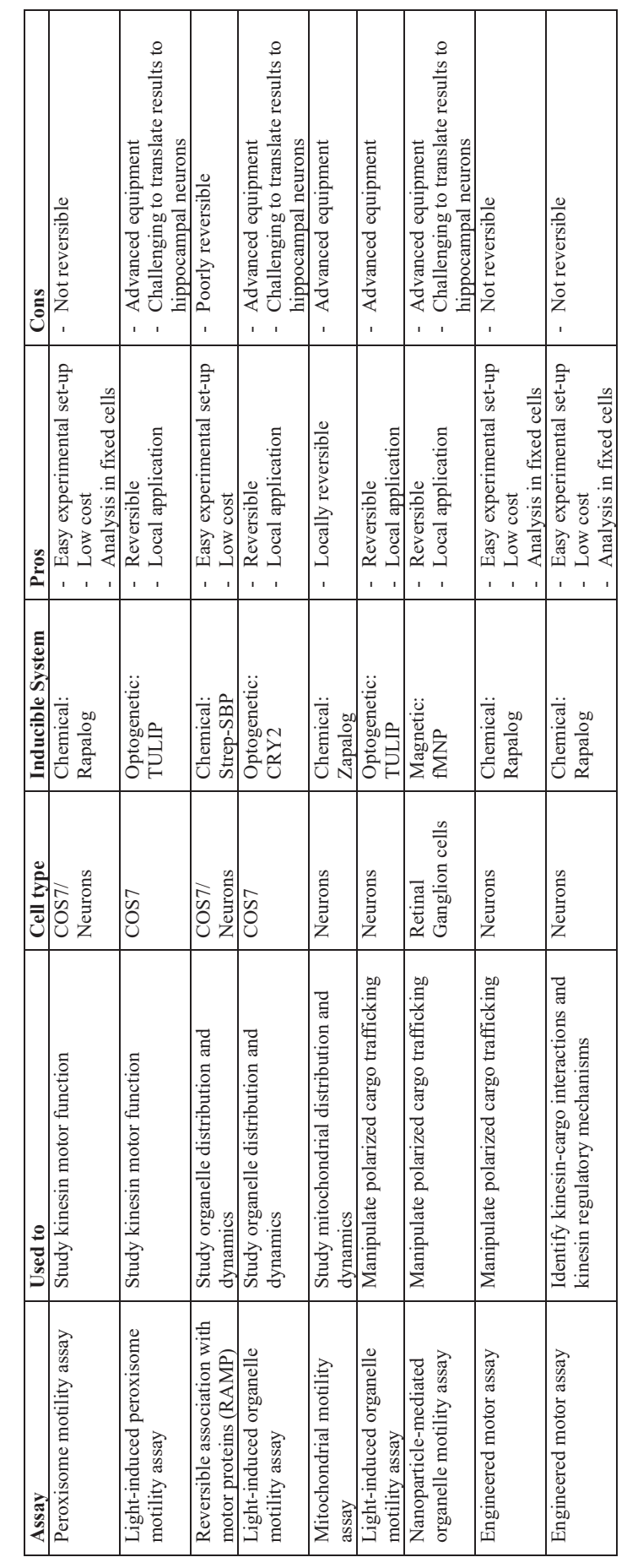

. 


\section{Addendum}

Nederlandse samenvatting voor non-experts

Curriculum vitae

List of publications

Dankwoord

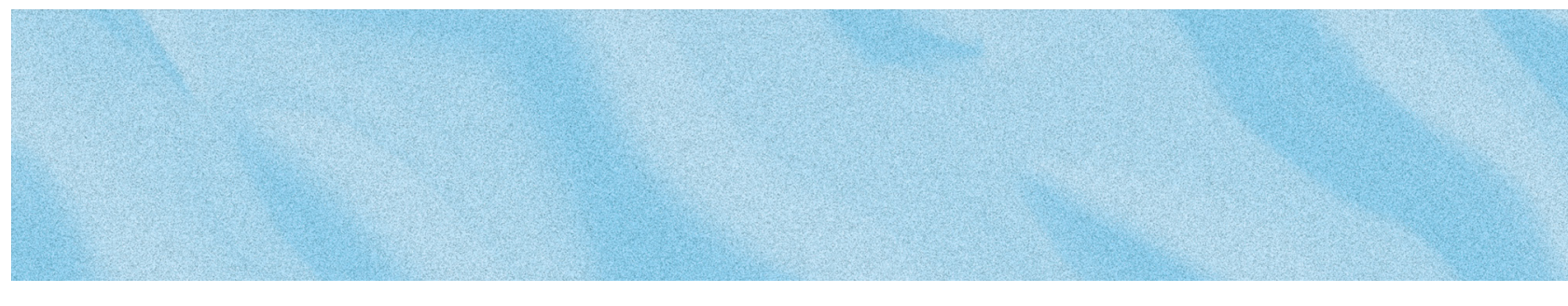




\section{NEDERLANDSE SAMENVATTING VOOR NON-EXPERTS}

Het brein is een zeer complex orgaan en ondanks jarenlang onderzoek is er nog steeds relatief weinig bekend over hoe ons brein functioneert. Het brein bestaat uit miljarden hersencellen, ook wel neuronen, die een intensief netwerk vormen. Dit netwerk reguleert onder andere hoe we bewegen en welke emoties we voelen, stuurt andere organen en autonome functies aan, en slaat herinneringen op. Een neuron bestaat uit twee compartimenten: een cellichaam me enkele korte vertakte uitlopers, ook wel dendrieten genoemd, en een lange vertakte uitloper, het axon. Neuronen communiceren met elkaar via elektrische signalen, die via het axon worden doorgegeven aan dendrieten van ernaast liggende cellen. Het contact tussen een axon van de ene cel en een dendriet van de andere cel wordt een synaps genoemd. In een synap

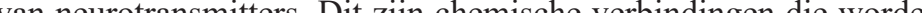
列 deze receptoren zorgt voor een verdere signaaltransductie in het volgende neuron. Efficiënte communicatie tussen hersencellen is van groot belang voor het functioneren van het gehele netwerk en defecten in deze communicatie kunnen leiden tot verschillende ziektebeelden.

Om de signaaltransductie van cel tot cel goed te kunnen laten verlopen, is het van belang dat elke cel de juiste bouwstenen op het juiste moment op de juiste plek heeft. Denk hierbij aan zakjes met neurotransmitters die aan het uiteinde van het axon moeten zitten of receptoren die zich op de dendriet moeten bevinden. Deze bouwstenen worden vaak gemaakt in het cellichaam en moeten dus getransporteerd worden naar de goede plek. Dit transport wordt uitgevoerd door zogenaamde motoreiwitten. Het onderzoek in dit proefschrift focust zich op een familie van motoreiwitten, de kinesines. Deze motortjes bewegen zich door de cel over de vele microtubuli. Dit zijn sterke bundels die stevigheid geven aan de cel en die fungeren als een netwerk van snelwegen voor een efficiënt transport. Een kinesine motortje bestaat uit twee delen: een deel dat loopt over de microtubuli, het motor deel, en een deel dat kan binden aan bouwstenen, het staart deel. Op deze manier kan een kinesine bouwstenen doo de cel transporteren naar de juiste plek. In hersencellen zijn er meer dan 40 verschillende soorten kinesines en nog veel meer verschilende soorten bouwstenen. Er is dan ook een complex transportsystem, waarin elke kinesine precies weet welke bouwseer cor getransporteerd moet worden. Maar hoe weet een kinesine welke bouwsteen hij moet meenemen? En waar hij deze dan vervolgens moet afleveren? En dat ook nog eens op het juiste moment? Dit zijn een aantal van de vraagstukken die ten grondslag liggen aan het onderzoek beschreven in dit proefschrift.

De beweging van kinesines kan op verschillende manieren gereguleerd worden, onder andere door inhiberende eiwitten. Een voorbeeld van zo een eiwit is KBP. Dit eiwit bind aan kinesines op het motor deel en voorkomt hierdoor dat de motor over de snelweg van A structuur van KBP in complex met de kinesines KIF15 en KIF1A opgehelderd. Begrip van de interactie tussen deze inhiberende eiwitten en de motortjes geeft ons inzicht in de regulatie van de verschillende kinesines.

Over de jaren heen zijn er verschillende technieken en methoden gebruikt om kinesines te bestuderen. In Hoofdstuk 3 wordt een nieuwe methode beschreven, de engineered moto assay, die geschikt is om transport door kinesines te onderzoeken. In deze methode wordt een kinesine opgesplitst in twee delen: het microtubuli-bindende motor deel en de bouwsteenbindende staart. De methode gebruikt het motor deel van de kinesine KIF5. Dit is een bijzondere motor, omdat bekend is dat deze altijd actief is en zich uitsluitend naar het uiteinde van het axon beweegt. Wanneer we een fluorescent label op dit motor deel zetten, kunnen we onder een microscoop zien dat dit motor deel accumuleert in het uiteinde van het axon.
Rapalog is een chemische verbinding, die twee domeinen, hier voor het gemak even X en Y, met elkaar kan verbinden. In de engineered motor assay wordt domein $\mathrm{X}$ aan het motor dee van KIF5 geplakt en domein $\mathrm{Y}$ aan het staart deel van een andere kinesine. Zonder rapalog zal het KIF5 motor deel naar het uiteinde van het axon bewegen, terwijl het staart deel zich (het heeft immers geen motor). Als rapalog aan de cel wordt toegevoegd, zal er een verbinding ontstaan tussen domein X en Y, waardoor het staart deel aan de motor vast komt te zitten en dus ook naar het uiteinde van het axon zal bewegen. Vervolgens kun je een bouwsteen met een fluorescent label aan het systeem toe te voegen. Als deze bouwsteen aan de staart van de gekozen kinesine bindt, zul je onder de microscoop zien dat deze doo de motor naar het uiteinde van het axon wordt getransporteerd. De engineered motor assay kan dus gebruikt worden om de interactie van verschillende kinesines met verschillende bouwstenen te onderzoeken.

De engineered motor assay heeft veel verschillende toepassingen in het onderzoek naar kinesines. Zo wordt deze methode in Hoofdstuk 4 gebruikt om meer inzicht te verkrijgen in de kinesine KIF1A. Allereerst zijn er met de assay drie verschillende bouwstenen van KIF1A geïdentificeerd. Dit leidt tot de vraag: hoe wordt er onderscheid gemaakt tussen deze verschillende bouwstenen? Een mogelijkheid hiervoor is dat de verschillende bouwstene aan een ander stukje van het staart deel binden. Om dit te onderzoeken wordt het staart deel opgeknipt in kleinere stukjes die elk aan domein Y worden vastgemaakt. Vervolgens kan met de engineered motor assay worden bekeken welk stukje van de staart kan binden aan de bouwsteen. Dit heeft laten zien dat er twee staartstukjes nodig zijn voor deze interactie, maar ook dat deze twee stukjes hetzelfde zijn voor alle drie de bouwstenen. Een andere mogelijkheid om specificiteit voor een bouwsteen te verkrijgen is via specifieke adapte eiwitten. Deze eiwitten kunnen aan zowel de kinesine als aan de bouwsteen binden en hierdoor de kinesine-bouwsteen interactie beïnvloeden. In Hoofdstuk 4 worden verschillende adapter eiwitten van KIF1A aan domein Y geplakt om vervolgens met de engineered moto assay te onderzoeken welke adapter met welke bouwsteen interacteert. Dit heeft laten zien dat specifieke adapters aan specifieke bouwstenen binden. Al met al hebben deze resultaten geleid tot een model van regulatie voor KIF1A. In dit model kan KIF1A aan verschillende bouwstenen binden. De voorkeur van KIF1A voor een bepaalde bouwsteen wordt bepaald door de aanwezigheid van specifieke adapter eiwitten, die ervoor zorgen dat de kinesinebouwsteen interactie sterk genoeg is voor het transport van de bouwsteen.

In Hoofdstuk 5 wordt gekeken naar het transport van een specifieke receptor, TrkB. Deze receptor speelt een belangrijke rol in de signaaltransductie. Met verschillende technieken wordt gekeken naar hoe dit transport wordt gereguleerd, waarbij ook gebruik wordt gemaak van de engineered motor assay. Uit dit onderzoek blijkt dat het transport van deze receptor gereguleerd wordt door twee kinesines, KIF1A en KIF5, die met elkaar samenwerken om de receptor binnen het axon te transporteren.

Samenvattend heeft het onderzoek beschreven in dit proefschrift geleid tot een beter begrip van de regulatie van kinesine motoren, met name van de kinesine KIF1A. De opheldering van de structuur van het inhiberende eiwit KBP in complex met KIF1A zorgt ervoor dat we beter kunnen begrijpen hoe de motor wordt gereguleerd. Daarnaast wordt er met behulp van de nieuw ontwikkelde engineered motor assay laten zien dat KIF1A aan verschillende bouwstenen kan binden en dat de specificiteit hierin wordt bepaald door verschillende adapter eiwitten. Daarbij wordt ook laten zien dat KIF1A samenwerkt met de kinesine KIF5 in he transport van de TrkB receptor. Deze inzichten in de regulatie van KIF1A dragen bij aan de fundamentele kennis over de transportmechanismen in een hersencel. Dit is een belangrijke stap in het begrijpen van het transportnetwerk en uiteindelijk voor het oplossen van ziektes in de hersenen die veroorzaakt worden door verstoringen binnen dit netwerk. 


\section{CURRICULUM VITAE}

Jessica J. A. Hummel was born on September 11th 1992 in Zoetermeer. After receiving her VWO diploma in 2009, she started her bachelor Molecular Science and Technology at Leiden University and Technical University Delft and obtained her degree in 2012. She continued with a master Chemistry and Research at Leiden University, and she performed her major internship in the group of prof. dr. Mario van der Stelt, where she worked on the synthesis of new DAGL $\alpha$ inhibitors. Jessica did a second internship at the University of Melbourne in the group of prof. dr. Mark Rizzacasa, working on the synthesis of dethiosecoemestrin. She received her master's degree in 2014. After a year of working experience at SSL Leiden, she started a master Molecular Neuroscience at the University of Bristol. Here, she performed a research project with $\mathrm{dr}$. Oscar Cordero Llana, where she developed novel optogenetic tools e study mitophagy and lysosomar Cordero Llan. Jessica obained her second master's degree with to study nith distinction in 2016. She then started her PhD in the group of prof. dr. Casper Hoogenraad at the Department of Biology at Utrecht University of which the main results are described in this dissertation.

\section{LIST OF PUBLICATIONS}

Jessica J.A. Hummel, Casper C. Hoogenraad (2021). Inducible manipulation of motorcargo interaction using engineered kinesin motors. Journal of Cell Science, 134, jcs258776.

Jessica J.A. Hummel, Casper C. Hoogenraad (2021). Specific KIF1A-adaptor interactions control selective cargo recognition. Journal of Cell Biology, 220, e202105011.

Robin R. Buijs, Jessica J.A. Hummel, Mithila Burute, Anna Akhmanova, Lukas C. Kapitein, Casper C. Hoogenraad (2021). WDR47 protects neuronal microtubule minus ends from katanin-mediated severing. Cell Reports, 36, 109371.

Feline W Lindhout, Sybren Portegies, Robbelien Kooistra, Lotte J Herstel, Riccardo Stucchi, Jessica J A Hummel, Nicky Scheefhals, Eugene A Katrukha, Maarten Altelaar, Harold D MacGillavry, Corette J Wierenga, Casper C Hoogenraad (2021). Centrosome-mediated microtubule remodeling during axon formation in human iPSC-derived neurons. The EMBO journal, 40, e106798.

Eitan Erez Zahavi, Jessica J.A. Hummel, Yuhao Han, Riccardo Stucchi, Maarten Altelaar Casper C. Hoogenraad (2021). Combined kinesin-1 and kinesin-3 activity drives axonal trafficking of TrkB receptors in Rab6 carriers. Developmental Cell, 56, 494-508.

Joseph Atherton, Jessica JA Hummel, Natacha Olieric, Julia Locke, Alejandro Peña, Steven S Rosenfeld, Michel O Steinmetz, Casper C Hoogenraad, Carolyn A Moores (2020). The mechanism of kinesin inhibition by kinesin-binding protein. eLife, 9:e61481.

Dieudonnée van de Willige*, Jessica JA Hummel*, Celine Alkemade*, Olga I Kahn, Franco KC Au, Robert Z Qi, Marileen Dogterom, Gijsje H Koenderink, Casper C Hoogenraad, and Anna Akhmanova (2019). Cytolinker Gas2L1 regulates axon morphology through microtubule-modulated actin stabilization. Embo Reports, 20, e47732: 1-20.

Riccardo Stucchi, Gabriela Plucińska, Jessica J A Hummel, Eitan E Zahavi, Irune Guerra San Juan, Oleg Klykov, Richard A Scheltema, A F Maarten Altelaar, Casper C Hoogenraad (2018). Regulation of KIF1 A-driven dense core vesicle transport: $\mathrm{Ca}^{2+} \mathrm{CaM}$ controls DCV binding and Liprin- $\alpha /$ TANC2 recruits DCVs to postsynaptic sites. Cell Reports, 24, 685-700.

Alex Cameron, Brendan Fisher, Nicholas Fisk, Jessica Hummel, Jonathan M. White, Elizabeth H. Krenske, and Mark A. Rizzacasa (2015). Towards the Synthesis of Dihydrooxepino[4,3-b] pyrrole-Containing Natural Products via Cope Rearrangement of Vinyl Pyrrole Epoxides. Organic Letters, 7, 5998-6001.

Freek J. Janssen, Marc P. Baggelaar, Jessica J. A. Hummel, Herman S. Overkleeft, Benjamin F. Cravatt, Dale L. Boger, and Mario van der Stelt (2015). Comprehensive Analysis of Structure-Activity Relationships of $\alpha$-Ketoheterocycles as sn-1-Diacylglycerol Lipase $\alpha$ Inhibitors. Journal of Medicinal Chemistry, 58, 9742-9753 
Marc P. Baggelaar, Pascal J. P. Chameau, Vasudev Kantae, Jessica Hummel, Ku-Lung Hsu, Freek Janssen, Tom van der Wel, Marjolein Soethoudt, Hui Deng, Hans den Dulk, Marco Allarà, Bogdan I. Florea, Vincenzo Di Marzo, Wytse J. Wadman, Chris G. Kruse, Herman S. Overkleeft, Thomas Hankemeier, Taco R. Werkman, Benjamin F. Cravatt \& Mario van der Stelt (2015). Highly Selective, Reversible Inhibitor Identified by Comparative Chemoproteomics Modulates Diacylglycerol Lipase Activity in Neurons. Journal of the American Chemical Society, 137, 8851-8857.

Robert den Hartog, Mickey R. Harvey, Jessica J.A. Hummel, Sven T. van der Pol, Ilpo Mutikainen, Gerard A. van Albada, Elisabeth Bouwman (2011). Co(II), Zn(II) and Cu(II) compounds with 1,$4 ; 1,3$ or 1,2-bis-(benzimidazole-1-yl-methylene)-benzene as a flexible spacer ligand: Synthesis, characterization and X-ray structures of some polynuclear species; Inorganica Chimica Acta, 376, 664-670.

* Authors contributed equally

\section{PATENTS}

Janssen, F. J.; Baggelaar, M. P.; Hummel, J. J. A.; van Boeckel, C. A. A.; van der Stelt, M. European Patent Number EP 15169 052.6. Filing date 23 May 2016.

\section{DANKWOORD}

Stap voor stap bewandelen jij en ik ons levenspad. De PhD symboliseert voor mij een grote en belangrijke stap, waarin ik stap voor stap het pad van kinesines in het neuron heb kunnen bestuderen. Ik heb genoten van de wetenschap en veel geleerd over het doen van experimenten, het uitvoeren van kritische analyses, het schrijven van publicaties, en daarbij vele skills opgedaan en in mijn rugzak gestopt.

Vijf jaar geleden begon dit avontuur. Onzeker, maar vastberaden. Niet wetende hoe het pad er uit zou zien, ben ik de uitdaging aangegaan. De hobbels onderweg hebben me sterker gemaakt, ik heb veel over mezelf geleerd en ben gegroeid. Bovenal, ik ben in mezelf gaan geloven. Ook deze kracht zit nu in mijn rugzak en neem ik mee op mijn verdere levenspad. Waar dat heen gaat, dat is nog niet bekend. Maar één ding is zeker: I can do it!

Afgelopen jaren heb ik met veel mensen mogen samenwerken. Met sommige was er een korte interactie, waarna we allebei ons eigen pad vervolgden. Anderen hebben gedurende het hele $\mathrm{PhD}$ pad met mij meegelopen. Kort of lang, dat maakt niet uit. Al deze interacties hebben mij geholpen om over de hobbels op mijn pad te komen en maken dat ik sta waar ik zelf doen, maar je kunt het niet alleen. Hierbij wil ik iedereen die mij heeft geholpen tijdens mijn $\mathrm{PhD}$ enorm bedanken!

Ik ben nu klaar om verder te wandelen. Voor iedereen van wie mijn weg nu scheidt, dankjewe voor alles wat je voor mij hebt betekend de afgelopen jaren. En voor iedereen die verder met mij meewandelt: dankjewel en tot gauw!

Jessica 